\title{
Substrate-Controlled, One-Pot Synthesis: Access to Chiral Chroman-2-one and Polycyclic Derivatives
}

\author{
Xue-Li Sun, ${ }^{[a]}$ Ying-Han Chen, ${ }^{[a]}$ Dan-Yang Zhu, ${ }^{[a]}$ Yan Zhang, ${ }^{[b]}$ and Yan-Kai Liu* ${ }^{[a]}$
}

[a] Key Laboratory of Marine Drugs, Chinese Ministry of Education, School of Medicine and Pharmacy, Ocean University of China5 Yushan Lu, Qingdao 266003 (China)

[b] Hubei Key Laboratory for Processing and Application of Catalytic Materials, Huanggang Normal University, Huanggang 438000 (China)

E-mail: liuyankai@ouc.edu.cn

\section{Supporting Information}




\section{Table of contents}

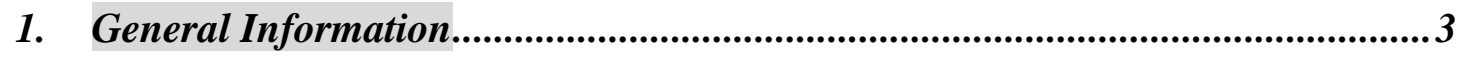

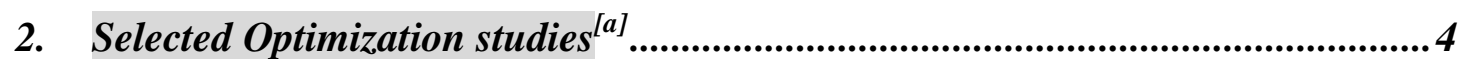

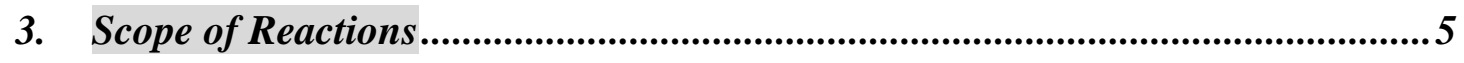

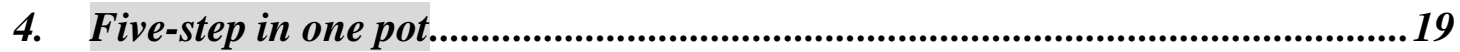

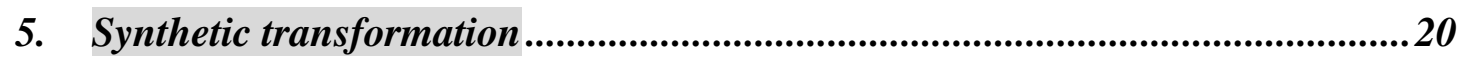

6. Single Crystal X-Ray Diffraction Data for Compound 11 ...............................27

7. NMR spectra and HPLC Traces .......................................................................28 


\section{General Information}

The ${ }^{1} \mathrm{H}$ and ${ }^{13} \mathrm{C}$ NMR spectra were recorded at $500 \mathrm{MHz}$ for ${ }^{1} \mathrm{H}$ and at $125 \mathrm{MHz}$ for ${ }^{13} \mathrm{C}$, respectively. The chemical shifts $(\delta)$ for ${ }^{1} \mathrm{H}$ and ${ }^{13} \mathrm{C}$ are given in ppm relative to residual signals of the solvents $\left(\mathrm{CDCl}_{3} @ 7.26 \mathrm{ppm}{ }^{1} \mathrm{H}\right.$ NMR, $\left.77.16 \mathrm{ppm}{ }^{13} \mathrm{C} \mathrm{NMR}\right)$. Coupling constants are given in $\mathrm{Hz}$. The following abbreviations are used to indicate the multiplicity: s, singlet; d, doublet; t, triplet; q, quartet; m, multiplet. High-resolution mass spectra (HRMS) were obtained from the Waters Q-Tof Ultima Global. X-ray data were obtained from Zhongke chemical technology service center. Optical rotations are reported as follows: $[a]_{\mathrm{D}}{ }^{20}$ (c in g per $100 \mathrm{~mL}$, solvent). All the reactions were set up under air and using freshly distilled solvents, without any precautions to exclude moisture, unless otherwise noted - open air chemistry on the benchtop. Chromatographic purification of products was accomplished using column chromatography on silica gel (300-400 mesh). For thin layer chromatography (TLC) analysis throughout this work, Merck precoated TLC plates (silica gel 60 GF254, $0.25 \mathrm{~mm}$ ) were used, using UV light as the visualizing agent and an acidic mixture of ceric ammonium molybdate or basic aqueous potassium permanganate $\left(\mathrm{KMnO}_{4}\right)$ as stain developing solutions. Organic solutions were concentrated under reduced pressure on a Büchi rotary evaporator. HPLC analyses on chiral stationary phase were performed on an Hitachi Chromaste. Daicel Chiralpak IA, IB , IC or OD-H columns with $i-\mathrm{PrOH} / n$-hexane as the eluent were used. HPLC traces were compared to racemic samples prepared by mixture of two enantiomeric final products obtained using $(S)$ and $(R)$ catalyst.

Materials : Commercial reagents and solvents were purchased from Sigma Aldrich, Fluka, and Alfa Aesar used as received, without further purification. $(S)$ and $(R)$-diphenylprolinol silyl ether is commercially available from Daicel chiral Technologies. All the (E)-3-(2-hydroxyphenyl)acrylaidehyde were synthesized from the corresponding Salicylaldehyde via Wittig reaction. The ethyl (E)-4-oxo-4-phenylbut-2-enoate were prepared from Ethyl glyoxalate and the corresponding 2-(triphenylphosphoranylidene)acetophenone in THF at the room temperature. 


\section{Selected Optimization studies ${ }^{[a]}$}

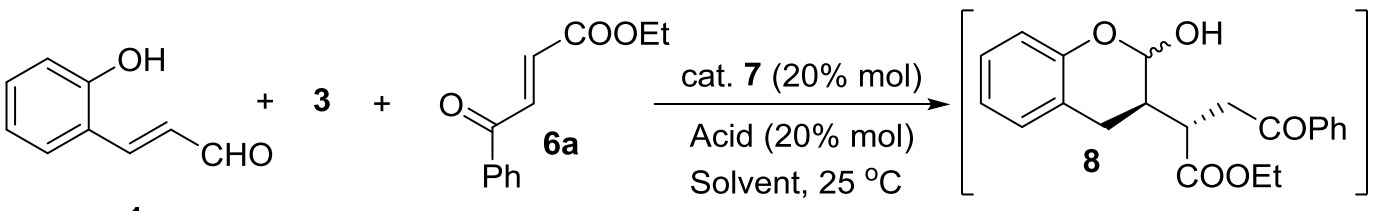

1a

Acid:<smiles>C[As](O)(c1ccccc1)C(c1ccccc1)(c1ccccc1)C1CCCN1</smiles>

cat. 7
$\mathrm{A}_{1}=\mathrm{PhCOOH}$

$\mathrm{A}_{2}=4-\mathrm{NO}_{2} \mathrm{PhCOOH}$

$\mathrm{A}_{3}=2-\mathrm{FPhCOOH}$

$\mathrm{A}_{4}=\left(\mathrm{CH}_{3}\right)_{3} \mathrm{CCOOH}$
PCC, DCM

$40^{\circ} \mathrm{C}, 4 \mathrm{~h}$

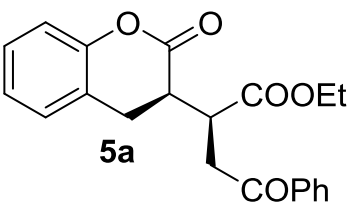

\begin{tabular}{cccccc}
\hline Entry $^{[a]}$ & Solvent & Acid & $\mathrm{t}[\mathrm{h}]^{[b]}$ & ${\text { Yield }[\%]^{[c]}}^{[c}$ & ee $[\%]^{[d]}$ \\
\hline 1 & toluene & $\mathrm{A}_{1}$ & 48 & 85 & 99.1 \\
2 & toluene & $\mathrm{A}_{2}$ & 48 & 84 & 98.9 \\
3 & toluene & $\mathrm{A}_{3}$ & 48 & 72 & 98.9 \\
4 & toluene & $\mathrm{A}_{4}$ & 48 & 87 & 99.0 \\
5 & $\mathrm{CH}_{3} \mathrm{CN}$ & $\mathrm{A}_{1}$ & 48 & 57 & 98.9 \\
$6^{[e]}$ & $\mathrm{CH}_{3} \mathrm{CN}$ & $\mathrm{A}_{1}$ & 48 & 65 & 99.7 \\
7 & $\mathrm{CH}_{2} \mathrm{Cl}$ & $\mathrm{A}_{1}$ & 48 & 84 & 98.9 \\
8 & $\mathrm{Et}_{2} \mathrm{O}$ & $\mathrm{A}_{1}$ & 48 & 88 & 99.3 \\
9 & $\mathrm{THF}$ & $\mathrm{A}_{1}$ & 48 & 33 & 99.1 \\
\hline
\end{tabular}

[a] Unless otherwise specified, all reactions were carried out using.1a ( $0.10 \mathrm{mmol}, 1.0$ equiv),

Hantzsch ester 3 ( $0.12 \mathrm{mmol}, 1.2$ equiv) and $\mathbf{6 a}(0.14 \mathrm{mmol}, 1.4$ equiv) in solvent ( $0.2 \mathrm{~mL})$ with cat. $7(20 \mathrm{~mol} \%)$ and acid $(20 \mathrm{~mol} \%)$ at $25{ }^{\circ} \mathrm{C}$. After $48 \mathrm{~h}$, the original solvent was replaced with $\mathrm{CH}_{2} \mathrm{Cl}_{2}(1.0 \mathrm{ml})$, the $\mathrm{PCC}(0.36 \mathrm{mmol}$, 3.6 equiv) with celite $(78 \mathrm{mg})$ were added, then the reaction was carried out at $40{ }^{\circ} \mathrm{C}$ for another $4 \mathrm{~h}$. [b] For the first step. [c] Isolated yields of product 5a. [d] Determined by chiral HPLC analysis of product 5a. [e] 10\% (v/v) $\mathrm{H}_{2} \mathrm{O}$ was added. $\mathrm{PCC}=$ pyridine chlorochromate. TMS = trimethylsilyl. $\mathrm{THF}=$ tetrahydrofuran. 


\section{Scope of Reactions}

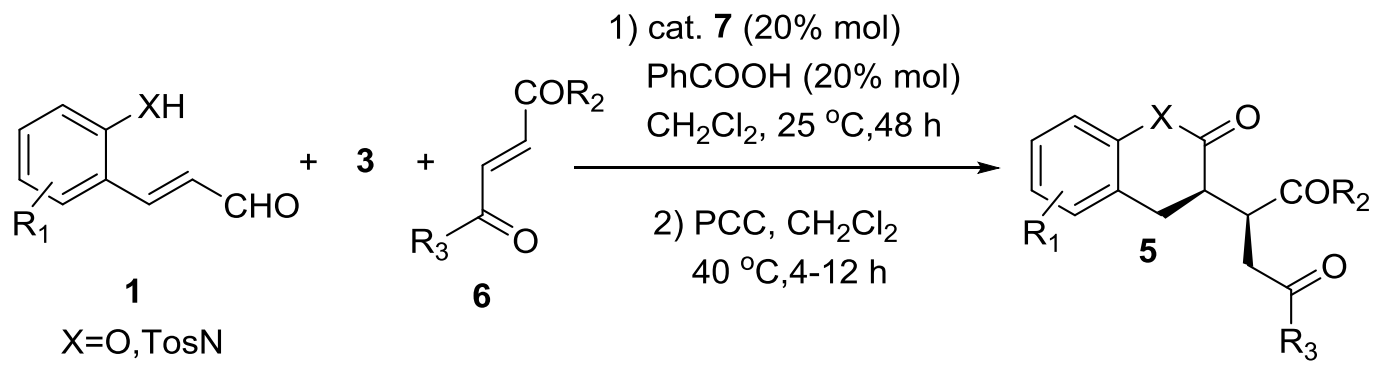

General procedure: A glass vial equipped with a magnetic stirring bar was charged with 1 (0.10 mmol, 1 equiv), Hantzsch ester 3 (30.4 mg, $0.12 \mathrm{mmol})$, electrophile 6 (0.14 mmol, 1.4 equiv ), cat. 7 (6.5 mg, $0.02 \mathrm{mmol})$, and $\mathrm{PhCOOH}(2.4 \mathrm{mg}, 0.02 \mathrm{mmol})$ in $\mathrm{CH}_{2} \mathrm{Cl}_{2}(0.2 \mathrm{~mL})$ at $25{ }^{\circ} \mathrm{C}$. After $48 \mathrm{~h}, \mathrm{PCC}(78 \mathrm{mg}, 0.36 \mathrm{mmol})$ with celite $(78 \mathrm{mg})$ and additional $\mathrm{CH}_{2} \mathrm{Cl}_{2}(0.8 \mathrm{ml})$ were added, then the reaction was stirred at $40{ }^{\circ} \mathrm{C}$ for another several hours. After the reaction was completed, the reaction mixture was filtrated through a short plug of silica gel. Solvent was removed under reduced pressure and the crude product purified by column chromatography on silica gel to provide the desired product 5 .

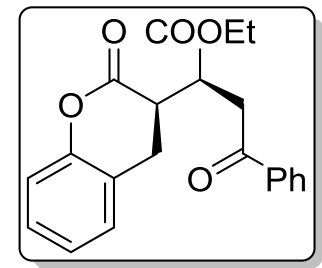

(S)-3-oxo-1-((R)-2-oxochroman-3-yl)-3-phenylpropyl propionate (5a)

5a was obtained as a colorless oil $29.6 \mathrm{mg}$ in $\mathbf{8 4 \%}$ yield for two steps after column chromatography on silica gel with petroleum ether-ethyl acetate $\left(15: 1, \mathbf{R}_{f}=0.1\right)$ as eluant. ${ }^{1} \mathbf{H}$ NMR $\left(500 \mathrm{MHz}, \mathrm{CDCl}_{3}\right) \delta 8.00(\mathrm{~d}, J=8.1 \mathrm{~Hz}, 2 \mathrm{H}), 7.58(\mathrm{t}, J=7.3 \mathrm{~Hz}, 1 \mathrm{H}), 7.48(\mathrm{t}, J=7.6$ $\mathrm{Hz}, 2 \mathrm{H}), 7.26(\mathrm{t}, J=7.6 \mathrm{~Hz}, 1 \mathrm{H}), 7.21-7.15(\mathrm{~m}, 1 \mathrm{H}), 7.10(\mathrm{t}, J=7.4 \mathrm{~Hz}, 1 \mathrm{H}), 7.07-7.03(\mathrm{~m}$ 1H), 4.19 (q, $J=7.1 \mathrm{~Hz}, 2 \mathrm{H}), 3.87(\mathrm{dd}, J=17.9,7.7 \mathrm{~Hz}, 1 \mathrm{H}), 3.66(\mathrm{dd}, J=12.8,5.5 \mathrm{~Hz}, 1 \mathrm{H})$, $3.44-3.36(\mathrm{~m}, 1 \mathrm{H}), 3.21(\mathrm{dd}, J=17.9,5.1 \mathrm{~Hz}, 1 \mathrm{H}), 3.05-2.93(\mathrm{~m}, 2 \mathrm{H}), 1.24(\mathrm{t}, J=7.2 \mathrm{~Hz}$, 3H). ${ }^{13} \mathrm{C}$ NMR $\left(125 \mathrm{MHz}, \mathrm{CDCl}_{3}\right) \delta 197.75,172.70,169.23,151.36,136.46,133.58,128.80$, $128.62,128.51,128.30,128.23,124.71,122.87,122.31,116.80,61.52,40.96,40.08,37.42$, 27.75, 14.20. HRMS: $[\mathrm{M}+\mathrm{H}]^{+}$calcd. For $\mathrm{C}_{21} \mathrm{H}_{21} \mathrm{O}_{5} \mathrm{~m} / \mathrm{z}$ : 353.1389; found: 353.1388. $[\alpha]_{\mathbf{D}}{ }^{20}$ $+24.4\left(c=0.17\right.$ in $\left.\mathrm{CHCl}_{3}\right)$. The enantiomeric excess was determined by HPLC analysis on 
Daicel Chiralpak IC column ( $n$-hexane $/ i-\mathrm{PrOH}=80 / 20,1 \mathrm{~mL} / \mathrm{min}), \lambda=210 \mathrm{~nm}, t_{\text {major }}=31.01$ $\min , t_{\text {minor }}=28.21 \mathrm{~min}, \boldsymbol{e e}=\mathbf{9 9 . 5 \%}$.

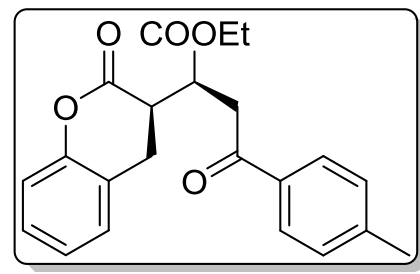

(S)-3-oxo-1-((R)-2-oxochroman-3-yl)-3-(p-tolyl)propyl propionate (5b)

5b was obtained as a colorless oil $24.5 \mathrm{mg}$ in $\mathbf{6 7 \%}$ yield for two steps after column chromatography on silica gel with petroleum ether-ethyl acetate $\left(12: 1, \mathbf{R}_{f}=0.1\right)$ as eluant. ${ }^{1} \mathbf{H}$ NMR $\left(500 \mathrm{MHz}, \mathrm{CDCl}_{3}\right) \delta 7.89(\mathrm{~d}, J=8.2 \mathrm{~Hz}, 2 \mathrm{H}), 7.29-7.23(\mathrm{~m}, 3 \mathrm{H}), 7.17(\mathrm{~d}, J=7.3 \mathrm{~Hz}$, $1 \mathrm{H}), 7.10(\mathrm{t}, J=7.4 \mathrm{~Hz}, 1 \mathrm{H}), 7.05(\mathrm{~d}, J=8.1 \mathrm{~Hz}, 1 \mathrm{H}), 4.18(\mathrm{q}, J=7.1 \mathrm{~Hz}, 2 \mathrm{H}), 3.83(\mathrm{dd}, J=$ 17.8, $7.5 \mathrm{~Hz}, 1 \mathrm{H}), 3.68-3.62(\mathrm{~m}, 1 \mathrm{H}), 3.43-3.35(\mathrm{~m}, 1 \mathrm{H}), 3.18(\mathrm{dd}, J=17.8,5.3 \mathrm{~Hz}, 1 \mathrm{H})$, $3.05-2.92(\mathrm{~m}, 2 \mathrm{H}), 2.41(\mathrm{~s}, 3 \mathrm{H}), 1.24(\mathrm{t}, J=7.1 \mathrm{~Hz}, 3 \mathrm{H}) .{ }^{13} \mathbf{C} \mathbf{~ N M R}\left(125 \mathrm{MHz}, \mathrm{CDCl}_{3}\right) \delta$ $197.32,172.78,169.27,151.38,144.45,134.00,129.48,128.59,128.43,128.33,124.69$, 122.36, 116.80, 61.48, 40.97, 40.06, 37.31, 27.71, 21.81, 14.21. HRMS: $[\mathrm{M}+\mathrm{H}]^{+}$calcd. For $\mathrm{C}_{22} \mathrm{H}_{23} \mathrm{O}_{5} \mathrm{~m} / \mathrm{z}: 367.1545$; found: $367.1548 .[\alpha]_{\mathbf{D}}{ }^{20}+6.6\left(c=1.67\right.$ in $\left.\mathrm{CHCl}_{3}\right)$. The enantiomeric excess was determined by HPLC analysis on Daicel Chiralpak IC column ( $n$-hexane/i-PrOH $=80 / 20,1 \mathrm{~mL} / \mathrm{min}), \lambda=210 \mathrm{~nm}, t_{\text {major }}=42.28 \mathrm{~min}, t_{\text {minor }}=38.82 \mathrm{~min}, \boldsymbol{e} \boldsymbol{e}=\mathbf{9 8 . 2 \%}$.

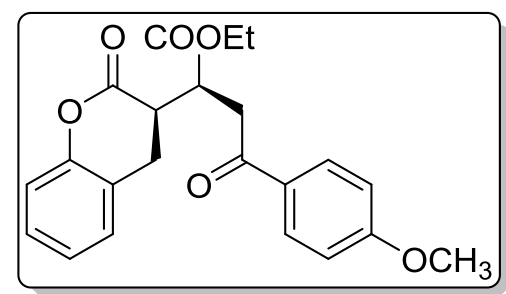

(S)-3-(4-methoxyphenyl)-3-oxo-1-((R)-2-oxochroman-3-yl)propyl propionate (5c)

5c was obtained as a colorless oil $28.8 \mathrm{mg}$ in $\mathbf{7 5 \%}$ yield for two steps after column chromatography on silica gel with petroleum ether-ethyl acetate $\left(10: 1, \mathbf{R}_{f}=0.1\right)$ as eluant. ${ }^{1} \mathbf{H}$ NMR $\left(500 \mathrm{MHz}, \mathrm{CDCl}_{3}\right) \delta 7.97(\mathrm{t}, J=9.5 \mathrm{~Hz}, 2 \mathrm{H}), 7.26(\mathrm{t}, J=7.6 \mathrm{~Hz}, 1 \mathrm{H}), 7.17(\mathrm{~d}, J=7.3$ $\mathrm{Hz}, 1 \mathrm{H}), 7.09$ (t, $J=7.4 \mathrm{~Hz}, 1 \mathrm{H}), 7.04(\mathrm{~d}, J=8.0 \mathrm{~Hz}, 1 \mathrm{H}), 6.94(\mathrm{~d}, J=8.9 \mathrm{~Hz}, 2 \mathrm{H}), 4.18(\mathrm{q}, J$ $=7.1 \mathrm{~Hz}, 2 \mathrm{H}), 3.87(\mathrm{~s}, 3 \mathrm{H}), 3.80(\mathrm{dd}, J=17.7,7.5 \mathrm{~Hz}, 1 \mathrm{H}), 3.67-3.61(\mathrm{~m}, 1 \mathrm{H}), 3.42-3.34$ (m, 1H), $3.16(\mathrm{dd}, J=17.7,5.3 \mathrm{~Hz}, 1 \mathrm{H}), 3.05-2.90(\mathrm{~m}, 2 \mathrm{H}), 1.23(\mathrm{t}, J=7.1 \mathrm{~Hz}, 3 \mathrm{H}) .{ }^{13} \mathrm{C}$ 
NMR $\left(125 \mathrm{MHz}, \mathrm{CDCl}_{3}\right) \delta 196.15,172.83,169.29,163.89,151.37,130.61,129.53,128.57$, 128.32, 124.68, 122.37, 116.78, 113.94, 61.45, 55.64, 40.98, 40.09, 37.04, 27.69, 14.21. HRMS: $[\mathrm{M}+\mathrm{H}]^{+}$calcd. For $\mathrm{C}_{22} \mathrm{H}_{23} \mathrm{O}_{6} \mathrm{~m} / \mathrm{z}$ : 383.1495; found: $383.1496 .[\alpha]_{\mathbf{D}}{ }^{20}-1.3(c=1.83$ in $\mathrm{CHCl}_{3}$ ). The enantiomeric excess was determined by HPLC analysis on Daicel Chiralpak IC column $(n$-hexane $/ i-\mathrm{PrOH}=70 / 30,1 \mathrm{~mL} / \mathrm{min}), \lambda=210 \mathrm{~nm}, t_{\text {major }}=44.27 \mathrm{~min}, t_{\text {minor }}=40.41$ $\min , \boldsymbol{e e}=\mathbf{9 9 . 9 \%}$.

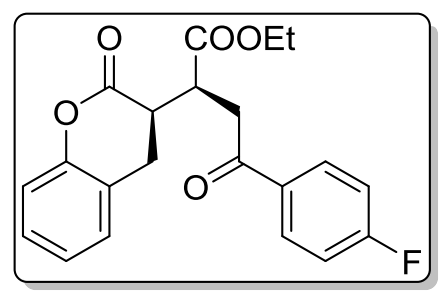

ethyl (S)-4-(4-fluorophenyl)-4-oxo-2-((R)-2-oxochroman-3-yl)butanoate (5d)

5d was obtained as a colorless oil $25 \mathrm{mg}$ in $\mathbf{6 7 \%}$ yield for two steps after column chromatography on silica gel with petroleum ether-ethyl acetate $\left(16: 1, \mathbf{R}_{f}=0.1\right)$ as eluant. ${ }^{1} \mathbf{H}$ NMR $\left(500 \mathrm{MHz}, \mathrm{CDCl}_{3}\right) \delta 8.07-7.98(\mathrm{~m}, 2 \mathrm{H}), 7.30-7.23(\mathrm{~m}, 1 \mathrm{H}), 7.20-7.08(\mathrm{~m}, 4 \mathrm{H})$, $7.05(\mathrm{~d}, J=8.0 \mathrm{~Hz}, 1 \mathrm{H}), 4.18(\mathrm{q}, J=6.9 \mathrm{~Hz}, 2 \mathrm{H}), 3.83(\mathrm{dd}, J=17.8,7.9 \mathrm{~Hz}, 1 \mathrm{H}), 3.66-3.58$ (m, 1H), $3.44-3.35(\mathrm{~m}, 1 \mathrm{H}), 3.15(\mathrm{dd}, J=17.8,4.3 \mathrm{~Hz}, 1 \mathrm{H}), 3.05-2.91(\mathrm{~m}, 2 \mathrm{H}), 1.23(\mathrm{t}, J=$ $7.0 \mathrm{~Hz}, 3 \mathrm{H}) .{ }^{13} \mathbf{C} \mathbf{N M R}\left(125 \mathrm{MHz}, \mathrm{CDCl}_{3}\right) \delta 196.20,172.62,169.20,167.10,165.07,151.36$, 132.96, 132.93, 131.04, 130.96, 128.67, 128.33, 124.76, 116.83, 116.03, 115.85, 61.57, 40.98, 40.20, 37.32, 27.84, 14.21. HRMS: $[\mathrm{M}+\mathrm{H}]^{+}$calcd. For $\mathrm{C}_{21} \mathrm{H}_{20} \mathrm{FO}_{5} \mathrm{~m} / \mathrm{z}$ : 371.1295; found: 371.1297. $[\boldsymbol{\alpha}]_{\mathbf{D}}{ }^{20}+10.6\left(c=1.25\right.$ in $\left.\mathrm{CHCl}_{3}\right)$. The enantiomeric excess was determined by HPLC analysis on Daicel Chiralpak IB column ( $n$-hexane $/ \mathrm{i}-\mathrm{PrOH}=98 / 2,1 \mathrm{~mL} / \mathrm{min}), \lambda=210$ $\mathrm{nm}, t_{\text {major }}=45.43 \mathrm{~min}, t_{\text {minor }}=42.54 \mathrm{~min}, \boldsymbol{e} \boldsymbol{e}=\mathbf{9 9 . 9 \%}$.

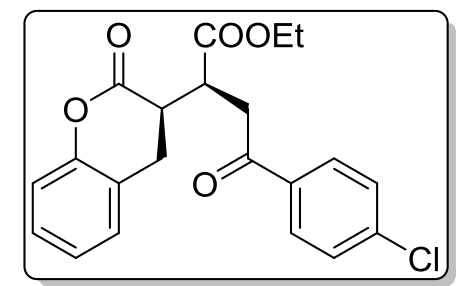

ethyl (S)-4-(4-chlorophenyl)-4-oxo-2-((R)-2-oxochroman-3-yl)butanoate (5e)

5e was obtained as a colorless oil $23.2 \mathrm{mg}$ in $\mathbf{6 0 \%}$ yield for two steps after column chromatography on silica gel with petroleum ether-ethyl acetate $\left(20: 1, \mathrm{R}_{f}=0.1\right)$ as eluant. ${ }^{1} \mathbf{H}$ NMR $\left(500 \mathrm{MHz}, \mathrm{CDCl}_{3}\right) \delta 7.93(\mathrm{~d}, J=8.1 \mathrm{~Hz}, 2 \mathrm{H}), 7.44(\mathrm{~d}, J=8.0 \mathrm{~Hz}, 2 \mathrm{H}), 7.30-7.23(\mathrm{~m}$, 
1H), $7.17(\mathrm{~d}, J=7.2 \mathrm{~Hz}, 1 \mathrm{H}), 7.10(\mathrm{t}, J=7.3 \mathrm{~Hz}, 1 \mathrm{H}), 7.05(\mathrm{~d}, J=8.0 \mathrm{~Hz}, 1 \mathrm{H}), 4.18(\mathrm{q}, J=$ $6.9 \mathrm{~Hz}, 2 \mathrm{H}), 3.83(\mathrm{dd}, J=17.8,7.9 \mathrm{~Hz}, 1 \mathrm{H}), 3.65-3.58(\mathrm{~m}, 1 \mathrm{H}), 3.43-3.35(\mathrm{~m}, 1 \mathrm{H}), 3.14$ $(\mathrm{dd}, J=17.8,4.3 \mathrm{~Hz}, 1 \mathrm{H}), 3.05-2.91(\mathrm{~m}, 2 \mathrm{H}), 1.23(\mathrm{t}, J=7.0 \mathrm{~Hz}, 3 \mathrm{H}) .{ }^{13} \mathbf{C} \mathbf{N M R}(125 \mathrm{MHz}$, $\left.\mathrm{CDCl}_{3}\right) . \delta 196.62,172.55,169.17,151.35,140.06,134.82,129.74,129.14,128.69,128.32$, 124.77, 122.22, 116.83, 61.60, 40.97, 40.20, 37.35, 27.85, 14.21. HRMS: $[\mathrm{M}+\mathrm{H}]^{+}$calcd. For $\mathrm{C}_{21} \mathrm{H}_{20} \mathrm{ClO}_{5} \mathrm{~m} / \mathrm{z}$ : 387.0999; found: 387.0998. $[\alpha]_{\mathbf{D}}{ }^{20}+0.3\left(c=1.25\right.$ in $\left.\mathrm{CHCl}_{3}\right)$. The enantiomeric excess was determined by HPLC analysis on Daicel Chiralpak IA column $(n$-hexane $/ i-\mathrm{PrOH}=80 / 20,1 \mathrm{~mL} / \mathrm{min}), \lambda=210 \mathrm{~nm}, t_{\text {major }}=40.63 \mathrm{~min}, t_{\text {minor }}=15.25 \mathrm{~min}, \boldsymbol{e} \boldsymbol{e}=$ $99.1 \%$.

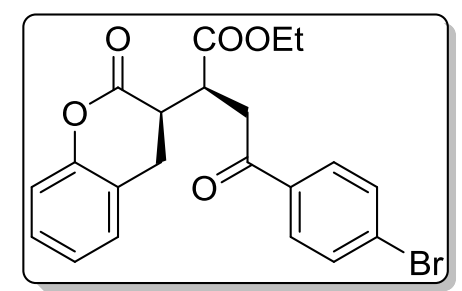

ethyl (S)-4-(4-bromophenyl)-4-oxo-2-((R)-2-oxochroman-3-yl)butanoate (5f)

5f was obtained as a colorless oil $34.1 \mathrm{mg}$ in $\mathbf{7 9 \%}$ yield for two steps after column chromatography on silica gel with petroleum ether-ethyl acetate $\left(21: 1, \mathbf{R}_{f}=0.1\right)$ as eluant. ${ }^{1} \mathbf{H}$ NMR $\left(500 \mathrm{MHz}, \mathrm{CDCl}_{3}\right) \delta 7.86(\mathrm{~d}, J=8.5 \mathrm{~Hz}, 2 \mathrm{H}), 7.61(\mathrm{~d}, J=8.5 \mathrm{~Hz}, 2 \mathrm{H}), 7.30-7.24(\mathrm{~m}$, 1H), $7.18(\mathrm{~d}, J=7.3 \mathrm{~Hz}, 1 \mathrm{H}), 7.10(\mathrm{t}, J=7.3 \mathrm{~Hz}, 1 \mathrm{H}), 7.05(\mathrm{~d}, J=8.1 \mathrm{~Hz}, 1 \mathrm{H}), 4.18(\mathrm{q}, J=$ $7.1 \mathrm{~Hz}, 2 \mathrm{H}), 3.82(\mathrm{dd}, J=17.8,8.0 \mathrm{~Hz}, 1 \mathrm{H}), 3.65-3.58(\mathrm{~m}, 1 \mathrm{H}), 3.43-3.36(\mathrm{~m}, 1 \mathrm{H}), 3.14$ $(\mathrm{dd}, J=17.8,4.8 \mathrm{~Hz}, 1 \mathrm{H}), 3.04-2.91(\mathrm{~m}, 2 \mathrm{H}), 1.23(\mathrm{t}, J=7.1 \mathrm{~Hz}, 3 \mathrm{H}) .{ }^{13} \mathbf{C}$ NMR $(125 \mathrm{MHz}$, $\left.\mathrm{CDCl}_{3}\right) \delta 196.83,172.54,169.17,151.35,135.22,132.13,129.84,128.80,128.69,128.32$, 124.77, 122.21, 116.83, 61.60, 40.97, 40.20, 37.32, 27.85, 14.21. HRMS: $[\mathrm{M}+\mathrm{H}]^{+}$calcd. For $\mathrm{C}_{21} \mathrm{H}_{20} \mathrm{BrO}_{5} \mathrm{~m} / \mathrm{z}$ : 431.0494; found: 431.0495. $[\boldsymbol{\alpha}]_{\mathbf{D}}{ }^{20}-1.0$ ( $c=1.67$ in $\left.\mathrm{CHCl}_{3}\right)$. The enantiomeric excess was determined by HPLC analysis on Daicel Chiralpak IC column $(n$-hexane $/ i-\mathrm{PrOH}=70 / 30,1 \mathrm{~mL} / \mathrm{min}), \lambda=210 \mathrm{~nm}, t_{\text {major }}=20.12 \mathrm{~min}, t_{\text {minor }}=21.76 \mathrm{~min}, \boldsymbol{e} \boldsymbol{e}=$ $99.6 \%$. 


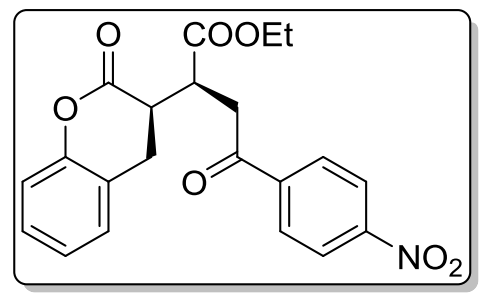

ethyl (S)-4-(4-nitrophenyl)-4-oxo-2-((R)-2-oxochroman-3-yl)butanoate (5g)

$\mathbf{5 g}$ was obtained as a light yellow oil $21 \mathrm{mg}$ in $\mathbf{5 3 \%}$ yield for two steps after column chromatography on silica gel with petroleum ether-ethyl acetate $\left(10: 1, \mathbf{R}_{f}=0.1\right)$ as eluant. ${ }^{1} \mathbf{H}$ NMR $\left(500 \mathrm{MHz}, \mathrm{CDCl}_{3}\right) \delta 8.32(\mathrm{~d}, J=8.8 \mathrm{~Hz}, 2 \mathrm{H}), 8.16(\mathrm{~d}, J=8.8 \mathrm{~Hz}, 2 \mathrm{H}), 7.31-7.25(\mathrm{~m}$, 1H), $7.19(\mathrm{~d}, J=7.3 \mathrm{~Hz}, 1 \mathrm{H}), 7.12(\mathrm{t}, J=7.4 \mathrm{~Hz}, 1 \mathrm{H}), 7.06(\mathrm{~d}, J=8.1 \mathrm{~Hz}, 1 \mathrm{H}), 4.19(\mathrm{q}, J=$ $7.1 \mathrm{~Hz}, 2 \mathrm{H}), 3.91(\mathrm{dd}, J=17.8,8.5 \mathrm{~Hz}, 1 \mathrm{H}), 3.65-3.58(\mathrm{~m}, 1 \mathrm{H}), 3.46-3.38(\mathrm{~m}, 1 \mathrm{H}), 3.18$ $(\mathrm{dd}, J=17.8,4.3 \mathrm{~Hz}, 1 \mathrm{H}), 3.06-2.94(\mathrm{~m}, 2 \mathrm{H}), 1.24(\mathrm{t}, J=7.1 \mathrm{~Hz}, 3 \mathrm{H}) .{ }^{13} \mathbf{C} \mathbf{N M R}(125 \mathrm{MHz}$, $\left.\mathrm{CDCl}_{3}\right) \delta 196.58,172.30,169.09,151.31,150.63,141.00,129.39,128.79,128.31,124.87$, 124.04, 122.06, 116.87, 61.82, 40.93, 40.46, 37.86, 27.92, 14.21. HRMS: $[\mathrm{M}+\mathrm{H}]^{+}$calcd. For $\mathrm{C}_{21} \mathrm{H}_{20} \mathrm{NO}_{7} \mathrm{~m} / \mathrm{z}$ : 398.1240; found: 398.1241. $[\alpha]_{\mathbf{D}}{ }^{20}+1.5\left(c=1.08\right.$ in $\left.\mathrm{CHCl}_{3}\right)$. The enantiomeric excess was determined by HPLC analysis on Daicel Chiralpak IC column ( $n$-hexane $/ i-\mathrm{PrOH}=60 / 40,1 \mathrm{~mL} / \mathrm{min}$ ), $\lambda=210 \mathrm{~nm}, t_{\text {major }}=44.02 \mathrm{~min}, t_{\text {minor }}=36.45 \mathrm{~min}, \boldsymbol{e} \boldsymbol{e}=$ $99.7 \%$.

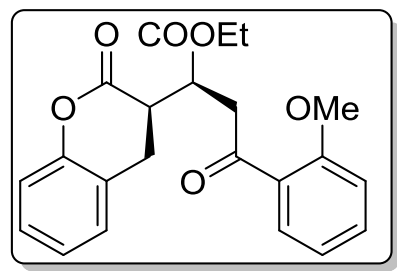

(S)-3-(2-methoxyphenyl)-3-oxo-1-((R)-2-oxochroman-3-yl)propyl propionate (5h)

5h was obtained as a colorless oil $23.5 \mathrm{mg}$ in $\mathbf{6 1 \%}$ yield for two steps after column chromatography on silica gel with petroleum ether-ethyl acetate $\left(10: 1, R_{f}=0.1\right)$ as eluant.

${ }^{1} \mathbf{H}$ NMR $\left(500 \mathrm{MHz}, \mathrm{CDCl}_{3}\right) \delta 7.72(\mathrm{~d}, J=6.5 \mathrm{~Hz}, 1 \mathrm{H}), 7.47(\mathrm{t}, J=8.3 \mathrm{~Hz}, 1 \mathrm{H}), 7.28-7.22$ (m, 1H), $7.17(\mathrm{~d}, J=7.3 \mathrm{~Hz}, 1 \mathrm{H}), 7.09$ (t, $J=7.4 \mathrm{~Hz}, 1 \mathrm{H}), 7.04$ (d, $J=8.1 \mathrm{~Hz}, 1 \mathrm{H}), 7.00(\mathrm{t}, J$ $=7.5 \mathrm{~Hz}, 1 \mathrm{H}), 6.96(\mathrm{~d}, J=8.4 \mathrm{~Hz}, 1 \mathrm{H}), 4.18(\mathrm{q}, J=7.1 \mathrm{~Hz}, 2 \mathrm{H}), 3.90(\mathrm{~s}, 3 \mathrm{H}), 3.73(\mathrm{dd}, J=$ 18.0, $7.3 \mathrm{~Hz}, 1 \mathrm{H}), 3.68-3.61(\mathrm{~m}, 1 \mathrm{H}), 3.37-3.34(\mathrm{~m}, 1 \mathrm{H}), 3.34-3.30(\mathrm{~m}, 1 \mathrm{H}), 3.07(\mathrm{t}, 1 \mathrm{H})$, $2.94(\mathrm{dd}, J=15.6,6.2 \mathrm{~Hz}, 1 \mathrm{H}), 1.24(\mathrm{t}, J=7.1 \mathrm{~Hz}, 3 \mathrm{H}) .{ }^{13} \mathbf{C}$ NMR $\left(125 \mathrm{MHz}, \mathrm{CDCl}_{3}\right) \delta$ $199.70,172.98,169.26,158.84,151.42,134.04,130.67,128.49,128.32,127.58,124.61$, 
$122.55,120.86,116.76,111.71,61.30,55.67,42.62,40.89,40.26,27.58,14.23$. HRMS: $[\mathrm{M}+\mathrm{H}]^{+}$calcd. For $\mathrm{C}_{22} \mathrm{H}_{23} \mathrm{O}_{6} \mathrm{~m} / \mathrm{z}: 383.1495$; found: 383.1497. $[\boldsymbol{\alpha}]_{\mathbf{D}}{ }^{20}+2.2\left(c=1.25\right.$ in $\left.\mathrm{CHCl}_{3}\right)$. The enantiomeric excess was determined by HPLC analysis on Daicel Chiralpak IC column $(n$-hexane $/ i-\mathrm{PrOH}=70 / 30,1 \mathrm{~mL} / \mathrm{min}), \lambda=210 \mathrm{~nm}, t_{\text {major }}=34.48 \mathrm{~min}, t_{\text {minor }}=28.90 \mathrm{~min}, \boldsymbol{e} \boldsymbol{e}=$ $99.3 \%$.

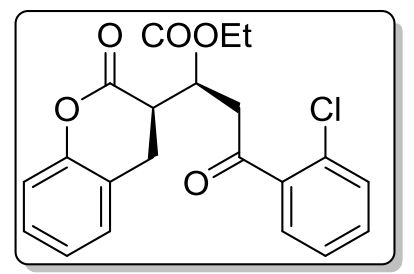

(S)-3-(2-chlorophenyl)-3-oxo-1-((R)-2-oxochroman-3-yl)propyl propionate (5i)

5i was obtained as a colorless oil $20 \mathrm{mg}$ in $\mathbf{5 1 \%}$ yield for two steps after column chromatography on silica gel with petroleum ether-ethyl acetate $\left(15: 1, \mathrm{R}_{f}=0.1\right)$ as eluant.

${ }^{1} \mathbf{H}$ NMR $\left(500 \mathrm{MHz}, \mathrm{CDCl}_{3}\right) \delta 7.60(\mathrm{~d}, J=7.9 \mathrm{~Hz}, 1 \mathrm{H}), 7.45-7.37(\mathrm{~m}, 2 \mathrm{H}), 7.37-7.31(\mathrm{~m}$, 1H), $7.30-7.24(\mathrm{~m}, 1 \mathrm{H}), 7.19$ (d, $J=7.3 \mathrm{~Hz}, 1 \mathrm{H}), 7.11(\mathrm{t}, J=7.4 \mathrm{~Hz}, 1 \mathrm{H}), 7.05$ (d, $J=8.1$ $\mathrm{Hz}, 1 \mathrm{H}), 4.19$ (q, $J=7.1 \mathrm{~Hz}, 2 \mathrm{H}), 3.77$ (dd, $J=18.1,8.3 \mathrm{~Hz}, 1 \mathrm{H}), 3.67-3.60$ (m, 1H), $3.45-$ $3.37(\mathrm{~m}, 1 \mathrm{H}), 3.18(\mathrm{dd}, J=18.1,4.6 \mathrm{~Hz}, 1 \mathrm{H}), 3.11-3.03(\mathrm{~m}, 1 \mathrm{H}), 2.98(\mathrm{dd}, J=15.6,6.3 \mathrm{~Hz}$, $1 \mathrm{H}), 1.25(\mathrm{t}, J=7.1 \mathrm{~Hz}, 3 \mathrm{H}) .{ }^{13} \mathbf{C}$ NMR $\left(125 \mathrm{MHz}, \mathrm{CDCl}_{3}\right) \delta 200.59,172.34,169.05,151.38$, $138.75,132.17,131.04,130.74,129.58,128.66,128.29,127.15,124.75,122.30,116.87$, 61.64, 41.31, 40.83, 40.42, 27.81, 14.23. HRMS: $[\mathrm{M}+\mathrm{H}]^{+}$calcd. For $\mathrm{C}_{21} \mathrm{H}_{20} \mathrm{ClO}_{5} \mathrm{~m} / \mathrm{z}$ : 387.0999; found: 387.0997 . $[\boldsymbol{\alpha}]_{\mathbf{D}}{ }^{20}-6.8\left(c=0.67\right.$ in $\left.\mathrm{CHCl}_{3}\right)$. The enantiomeric excess was determined by HPLC analysis on Daicel Chiralpak IC column ( $n$-hexane/i-PrOH $=80 / 20,1$ $\mathrm{mL} / \mathrm{min}), \lambda=210 \mathrm{~nm}, t_{\text {major }}=29.09 \mathrm{~min}, t_{\text {minor }}=26.77 \mathrm{~min}, \boldsymbol{e} \boldsymbol{e}=\mathbf{9 7 . 2 \%}$.

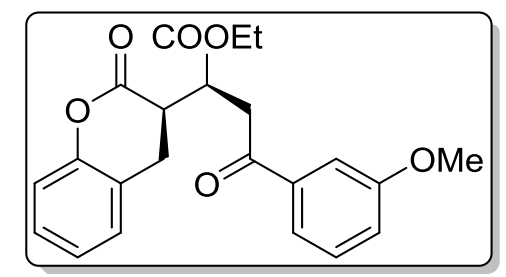

(S)-3-(3-methoxyphenyl)-3-oxo-1-((R)-2-oxochroman-3-yl)propyl propionate (5j)

5j was obtained as a colorless oil $26 \mathrm{mg}$ in $\mathbf{6 8 \%}$ yield for two steps after column chromatography on silica gel with petroleum ether-ethyl acetate $\left(15: 1, \mathrm{R}_{f}=0.1\right)$ as eluant. 
${ }^{1} \mathbf{H}$ NMR $\left(500 \mathrm{MHz}, \mathrm{CDCl}_{3}\right) \delta 7.59(\mathrm{~d}, J=7.7 \mathrm{~Hz}, 1 \mathrm{H}), 7.50(\mathrm{~d}, J=2.1 \mathrm{~Hz}, 1 \mathrm{H}), 7.38(\mathrm{t}, J=$ $7.9 \mathrm{~Hz}, 1 \mathrm{H}), 7.29-7.24(\mathrm{~m}, 1 \mathrm{H}), 7.17(\mathrm{~d}, J=7.3 \mathrm{~Hz}, 1 \mathrm{H}), 7.14-7.07(\mathrm{~m}, 2 \mathrm{H}), 7.05(\mathrm{~d}, J=$ $8.1 \mathrm{~Hz}, 1 \mathrm{H}), 4.19(\mathrm{q}, J=7.1 \mathrm{~Hz}, 2 \mathrm{H}), 3.85(\mathrm{~s}, 3 \mathrm{H}), 3.85-3.80(\mathrm{~m}, 1 \mathrm{H}), 3.68-3.61(\mathrm{~m}, 1 \mathrm{H})$, $3.42-3.35(\mathrm{~m}, 1 \mathrm{H}), 3.21(\mathrm{dd}, J=17.9,5.2 \mathrm{~Hz}, 1 \mathrm{H}), 3.05-2.91(\mathrm{~m}, 2 \mathrm{H}), 1.24(\mathrm{t}, J=7.1 \mathrm{~Hz}$, 3H). ${ }^{13} \mathrm{C}$ NMR $\left(125 \mathrm{MHz}, \mathrm{CDCl}_{3}\right) \delta 197.56,172.69,169.22,160.01,151.38,137.80,129.82$, $128.63,128.32,124.72,122.31,120.98,120.18,116.82,112.42,61.53,55.61,40.96,40.13$, 37.63, 27.74, 14.22. HRMS: $[\mathrm{M}+\mathrm{H}]^{+}$calcd. For $\mathrm{C}_{22} \mathrm{H}_{23} \mathrm{O}_{6} \mathrm{~m} / \mathrm{z}$ : 383.1495; found: 383.1496. $[\boldsymbol{\alpha}]_{\mathbf{D}}{ }^{\mathbf{2 0}}+11.4\left(c=0.83\right.$ in $\left.\mathrm{CHCl}_{3}\right)$. The enantiomeric excess was determined by HPLC analysis on Daicel Chiralpak IB column ( $n$-hexane $/ i$-PrOH $=80 / 20,1 \mathrm{~mL} / \mathrm{min}), \lambda=210 \mathrm{~nm}, t_{\text {major }}=$ $10.33 \mathrm{~min}, t_{\text {minor }}=9.21 \mathrm{~min}, \boldsymbol{e e}=\mathbf{9 9 . 3 \%}$.

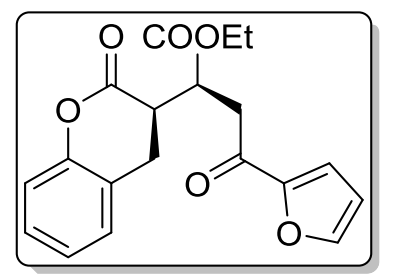

(S)-3-(furan-2-yl)-3-oxo-1-((R)-2-oxochroman-3-yl)propyl propionate (5k)

5k was obtained as a colorless oil $30 \mathrm{mg}$ in $\mathbf{7 7 \%}$ yield for two steps after column chromatography on silica gel with petroleum ether-ethyl acetate $\left(8: 1, \mathrm{R}_{f}=0.1\right)$ as eluant.

${ }^{1} \mathbf{H}$ NMR $\left(500 \mathrm{MHz}, \mathrm{CDCl}_{3}\right) \delta 7.59(\mathrm{~d}, J=8.7 \mathrm{~Hz}, 1 \mathrm{H}), 7.29-7.22(\mathrm{~m}, 2 \mathrm{H}), 7.17(\mathrm{~d}, J=7.1$ $\mathrm{Hz}, 1 \mathrm{H}), 7.10(\mathrm{t}, J=7.2 \mathrm{~Hz}, 1 \mathrm{H}), 7.05(\mathrm{~d}, J=8.1 \mathrm{~Hz}, 1 \mathrm{H}), 6.58-6.52(\mathrm{~m}, 1 \mathrm{H}), 4.20-4.13$ (m, 2H), $3.72(\mathrm{dd}, J=17.5,7.7 \mathrm{~Hz}, 1 \mathrm{H}), 3.66-3.60(\mathrm{~m}, 1 \mathrm{H}), 3.42-3.35(\mathrm{~m}, 1 \mathrm{H}), 3.09-$ $3.03(\mathrm{~m}, 1 \mathrm{H}), 3.00(\mathrm{~d}, J=12.8 \mathrm{~Hz}, 1 \mathrm{H}), 2.95(\mathrm{dd}, J=15.6,6.5 \mathrm{~Hz}, 1 \mathrm{H}), 1.22(\mathrm{t}, J=7.1 \mathrm{~Hz}$, 3H). ${ }^{13} \mathrm{C}$ NMR $\left(125 \mathrm{MHz}, \mathrm{CDCl}_{3}\right) \delta 186.69,172.44,169.14,152.29,151.37,146.81,128.62$, $128.29,124.71,122.34,117.74,116.82,112.53,61.56,40.89,39.69,29.83,27.66,14.15$. HRMS: $[\mathrm{M}+\mathrm{H}]^{+}$calcd. For $\mathrm{C}_{19} \mathrm{H}_{19} \mathrm{O}_{6} \mathrm{~m} / \mathrm{z}$ : 343.1182; found: 343.1183. $[\boldsymbol{\alpha}]_{\mathbf{D}}{ }^{20}+12.8(c=1.33$ in $\mathrm{CHCl}_{3}$ ). The enantiomeric excess was determined by HPLC analysis on Daicel Chiralpak IC column $(n$-hexane $/ i-\mathrm{PrOH}=70 / 30,1 \mathrm{~mL} / \mathrm{min}), \lambda=210 \mathrm{~nm}, t_{\text {major }}=45.42 \mathrm{~min}, t_{\text {minor }}=$ $35.95 \min , \boldsymbol{e e}=\mathbf{9 8 . 1 \%}$. 


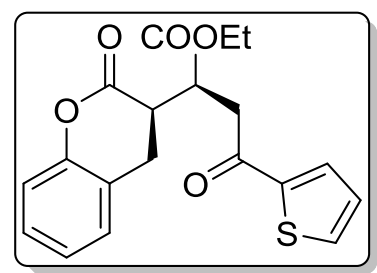

(S)-3-oxo-1-((R)-2-oxochroman-3-yl)-3-(thiophen-2-yl)propyl propionate (5I)

5l was obtained as a colorless oil $24 \mathrm{mg}$ in $\mathbf{6 7 \%}$ yield for two steps after column chromatography on silica gel with petroleum ether-ethyl acetate $\left(10: 1, \mathbf{R}_{f}=0.1\right)$ as eluant.

${ }^{1} \mathbf{H}$ NMR $\left(500 \mathrm{MHz}, \mathrm{CDCl}_{3}\right) \delta 7.79(\mathrm{dd}, J=14.1,3.7 \mathrm{~Hz}, 1 \mathrm{H}), 7.66(\mathrm{t}, J=4.7 \mathrm{~Hz}, 1 \mathrm{H}), 7.29-$ $7.23(\mathrm{~m}, 1 \mathrm{H}), 7.18(\mathrm{t}, J=7.2 \mathrm{~Hz}, 1 \mathrm{H}), 7.16-7.13(\mathrm{~m}, 1 \mathrm{H}), 7.10(\mathrm{t}, J=7.4 \mathrm{~Hz}, 1 \mathrm{H}), 7.04(\mathrm{t}, J$ $=7.1 \mathrm{~Hz}, 1 \mathrm{H}), 4.18(\mathrm{q}, J=7.1 \mathrm{~Hz}, 2 \mathrm{H}), 3.80(\mathrm{dd}, J=17.4,7.6 \mathrm{~Hz}, 1 \mathrm{H}), 3.66-3.60(\mathrm{~m}, 1 \mathrm{H})$, $3.44-3.37(\mathrm{~m}, 1 \mathrm{H}), 3.15(\mathrm{dd}, J=17.4,5.2 \mathrm{~Hz}, 1 \mathrm{H}), 3.06-2.92(\mathrm{~m}, 2 \mathrm{H}), 1.22(\mathrm{t}, J=7.1 \mathrm{~Hz}$, 3H). ${ }^{13} \mathrm{C}$ NMR $\left(125 \mathrm{MHz}, \mathrm{CDCl}_{3}\right) \delta 190.57,172.46,169.18,151.35,143.53,134.20,132.52$, 128.62, 128.37, 128.30, 124.72, 122.34, 116.81, 61.58, 40.91, 40.11, 37.76, 27.73, 14.16. HRMS: $[\mathrm{M}+\mathrm{H}]^{+}$calcd. For $\mathrm{C}_{19} \mathrm{H}_{19} \mathrm{O}_{5} \mathrm{~S} \mathrm{~m} / \mathrm{z}$ : 359.0953; found: 359.0954. $[\boldsymbol{\alpha}]_{\mathbf{D}}{ }^{20}+24.0(c=$ 1.67 in $\mathrm{CHCl}_{3}$ ). The enantiomeric excess was determined by HPLC analysis on Daicel Chiralpak IC column $(n$-hexane $/ i-\mathrm{PrOH}=80 / 20,1 \mathrm{~mL} / \mathrm{min}), \lambda=210 \mathrm{~nm}, t_{\text {major }}=53.17 \mathrm{~min}$, $t_{\text {minor }}=50.21 \mathrm{~min}, \boldsymbol{e e}=\mathbf{9 8 . 2 \%}$.

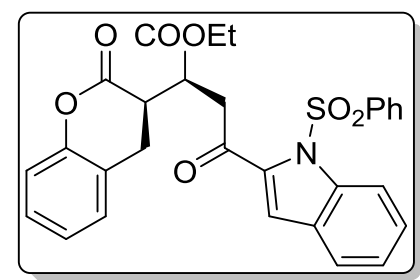

(S)-3-oxo-1-((R)-2-oxochroman-3-yl)-3-(1-(phenylsulfonyl)-1 H-indol-2-yl)propyl propionate (5m)

$\mathbf{5 m}$ was obtained as a white foam solid $28 \mathrm{mg}$ in $\mathbf{5 3 \%}$ yield for two steps after column chromatography on silica gel with petroleum ether-ethyl acetate $\left(12: 1, \mathbf{R}_{f}=0.1\right)$ as eluant.

${ }^{1} \mathbf{H}$ NMR $\left(500 \mathrm{MHz}, \mathrm{CDCl}_{3}\right) \delta 8.36-8.25(\mathrm{~m}, 2 \mathrm{H}), 7.97(\mathrm{dd}, J=15.1,8.0 \mathrm{~Hz}, 3 \mathrm{H}), 7.61(\mathrm{t}, J$ $=7.4 \mathrm{~Hz}, 1 \mathrm{H}), 7.51(\mathrm{t}, J=7.8 \mathrm{~Hz}, 2 \mathrm{H}), 7.42-7.31(\mathrm{~m}, 2 \mathrm{H}), 7.30-7.23(\mathrm{~m}, 1 \mathrm{H}), 7.22-7.16$ $(\mathrm{m}, 1 \mathrm{H}), 7.10(\mathrm{t}, J=7.4 \mathrm{~Hz}, 1 \mathrm{H}), 7.05(\mathrm{t}, J=8.2 \mathrm{~Hz}, 1 \mathrm{H}), 4.18(\mathrm{q}, J=7.0 \mathrm{~Hz}, 2 \mathrm{H}), 3.80(\mathrm{dd}, J$ $=17.3,7.8 \mathrm{~Hz}, 1 \mathrm{H}), 3.70-3.61(\mathrm{~m}, 1 \mathrm{H}), 3.47-3.38(\mathrm{~m}, 1 \mathrm{H}), 3.15(\mathrm{dd}, J=17.3,4.9 \mathrm{~Hz}, 1 \mathrm{H})$, $3.08-2.94(\mathrm{~m}, 2 \mathrm{H}), 1.22(\mathrm{t}, J=7.1 \mathrm{~Hz}, 3 \mathrm{H}) .{ }^{13} \mathbf{C} \mathbf{~ N M R}\left(125 \mathrm{MHz}, \mathrm{CDCl}_{3}\right) \delta 193.17,172.59$, $169.28,151.36,137.63,135.02,134.74,132.15,129.81,128.65,128.33,127.60,127.30$, 
$126.03,125.07,124.75,123.16,122.29,120.84,116.83,113.22,61.58,40.98,39.88,38.51$,

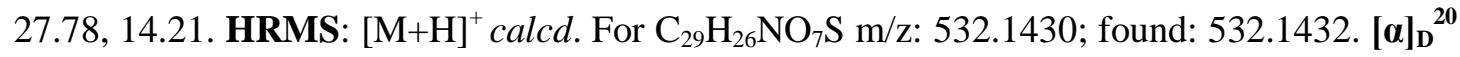
$+22.9\left(c=1.33\right.$ in $\left.\mathrm{CHCl}_{3}\right)$. The enantiomeric excess was determined by HPLC analysis on Daicel Chiralpak IC column ( $n$-hexane $/ i-\mathrm{PrOH}=70 / 30,1 \mathrm{~mL} / \mathrm{min}), \lambda=210 \mathrm{~nm}, t_{\text {major }}=49.22$ $\min , t_{\text {minor }}=55.90 \mathrm{~min}, \boldsymbol{e} \boldsymbol{e}=\mathbf{9 9 . 4 \%}$.

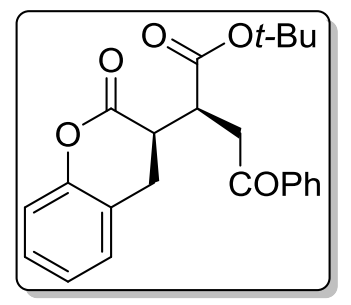

tert-butyl (S)-4-oxo-2-((R)-2-oxochroman-3-yl)-4-phenylbutanoate (5n)

5n was obtained as a colorless oil $20.2 \mathrm{mg}$ in $\mathbf{5 3 \%}$ yield for two steps after column chromatography on silica gel with petroleum ether-ethyl acetate $\left(16: 1, \mathbf{R}_{f}=0.1\right)$ as eluant. ${ }^{1} \mathbf{H}$ NMR $\left(500 \mathrm{MHz}, \mathrm{CDCl}_{3}\right) \delta 7.99(\mathrm{t}, J=7.7 \mathrm{~Hz}, 2 \mathrm{H}), 7.57(\mathrm{t}, J=7.4 \mathrm{~Hz}, 1 \mathrm{H}), 7.50-7.43(\mathrm{~m}$, 2H), $7.25(\mathrm{t}, J=10.2 \mathrm{~Hz}, 1 \mathrm{H}), 7.22-7.16(\mathrm{~m}, 1 \mathrm{H}), 7.10(\mathrm{t}, J=7.4 \mathrm{~Hz}, 1 \mathrm{H}), 7.07-7.02(\mathrm{~m}$, 1H), $3.83(\mathrm{dd}, J=17.8,7.8 \mathrm{~Hz}, 1 \mathrm{H}), 3.58-3.52(\mathrm{~m}, 1 \mathrm{H}), 3.38-3.29(\mathrm{~m}, 1 \mathrm{H}), 3.12(\mathrm{dd}, J=$ 17.8, 5.1 Hz, 1H), $3.05-2.93(\mathrm{~m}, 2 \mathrm{H}), 1.43(\mathrm{~s}, 9 \mathrm{H}) .{ }^{13} \mathbf{C}$ NMR $\left(125 \mathrm{MHz}, \mathrm{CDCl}_{3}\right) \delta$ 197.91, $171.68,169.27,151.43,136.62,133.49,128.79,128.77,128.58,128.34,128.31,128.24$, 124.66, 122.39, 116.83, 81.99, 41.11, 40.92, 37.43, 28.05, 27.81. HRMS: $[\mathrm{M}+\mathrm{H}]^{+}$calcd. For $\mathrm{C}_{23} \mathrm{H}_{25} \mathrm{O}_{5} \mathrm{~m} / \mathrm{z}: 381.1702$; found: 381.1703 . $[\boldsymbol{\alpha}]_{\mathbf{D}}{ }^{20}+7.0\left(c=0.21\right.$ in $\left.\mathrm{CHCl}_{3}\right)$. The enantiomeric excess was determined by HPLC analysis on Daicel Chiralpak IC column ( $n$-hexane/i-PrOH $=70 / 30,1 \mathrm{~mL} / \mathrm{min}), \lambda=210 \mathrm{~nm}, t_{\text {major }}=9.76 \mathrm{~min}, t_{\text {minor }}=8.95 \mathrm{~min}, \boldsymbol{e} \boldsymbol{e}=\mathbf{9 7 . 2 \%}$.

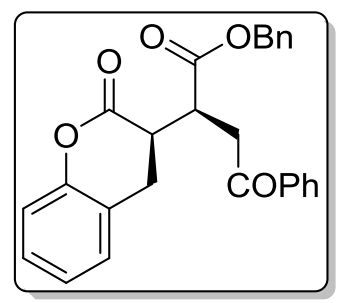

\section{benzyl (S)-4-oxo-2-((R)-2-oxochroman-3-yl)-4-phenylbutanoate (5o)}

5o was obtained as a colorless oil $28 \mathrm{mg}$ in $\mathbf{6 7 \%}$ yield for two steps after column chromatography on silica gel with petroleum ether-ethyl acetate $\left(15: 1, \mathbf{R}_{f}=0.1\right)$ as eluant. ${ }^{1} \mathbf{H}$ NMR $\left(500 \mathrm{MHz}, \mathrm{CDCl}_{3}\right) \delta 7.97(\mathrm{~d}, J=7.5 \mathrm{~Hz}, 2 \mathrm{H}), 7.58(\mathrm{t}, J=7.4 \mathrm{~Hz}, 1 \mathrm{H}), 7.46(\mathrm{t}, J=7.7$ $\mathrm{Hz}, 2 \mathrm{H}), 7.32(\mathrm{~d}, J=6.1 \mathrm{~Hz}, 4 \mathrm{H}), 7.26-7.22(\mathrm{~m}, 1 \mathrm{H}), 7.09-7.00(\mathrm{~m}, 3 \mathrm{H}), 5.22-5.13(\mathrm{~m}$, 
2H), $3.86(\mathrm{dd}, J=17.9,7.8 \mathrm{~Hz}, 1 \mathrm{H}), 3.74-3.67(\mathrm{~m}, 1 \mathrm{H}), 3.40-3.33(\mathrm{~m}, 1 \mathrm{H}), 3.26(\mathrm{dd}, J=$ 17.9, $4.9 \mathrm{~Hz}, 1 \mathrm{H}), 2.98-2.89(\mathrm{~m}, 1 \mathrm{H}), 2.85(\mathrm{dd}, J=15.6,6.3 \mathrm{~Hz}, 1 \mathrm{H}) .{ }^{13} \mathbf{C} \mathbf{N M R}(125 \mathrm{MHz}$, $\left.\mathrm{CDCl}_{3}\right) \delta 197.66,172.64,169.19,151.32,136.40,135.61,133.59,128.80,128.67,128.61$, $128.56,128.46,128.31,124.70,122.18,116.77,67.32,41.03,40.14,37.64,29.83$. HRMS: $[\mathrm{M}+\mathrm{H}]^{+}$calcd. For $\mathrm{C}_{26} \mathrm{H}_{23} \mathrm{O}_{5} \mathrm{~m} / \mathrm{z}: 415.1545$; found: 415.1546. $[\boldsymbol{\alpha}]_{\mathbf{D}}{ }^{20}-0.5\left(c=1.25\right.$ in $\left.\mathrm{CHCl}_{3}\right)$. The enantiomeric excess was determined by HPLC analysis on Daicel Chiralpak OD-H column $(n$-hexane $/ i-\mathrm{PrOH}=95 / 5,1 \mathrm{~mL} / \mathrm{min}), \lambda=210 \mathrm{~nm}, t_{\text {major }}=55.22 \mathrm{~min}, t_{\text {minor }}=65.67$ $\min , \boldsymbol{e} e=98.6 \%$.

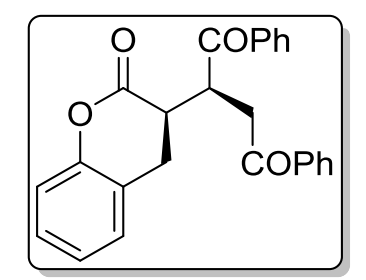

(S)-2-((R)-2-oxochroman-3-yl)-1,4-diphenylbutane-1,4-dione (5p)

5p was obtained as a white solid $25 \mathrm{mg}$ in $\mathbf{6 8 \%}$ yield for two steps after column chromatography on silica gel with petroleum ether-ethyl acetate $\left(15: 1, \mathrm{R}_{f}=0.1\right)$ as eluant. ${ }^{1}$ H NMR (500 MHz, DMSO) $\delta 7.96(\mathrm{t}, J=6.8 \mathrm{~Hz}, 4 \mathrm{H}), 7.68-7.61(\mathrm{~m}, 2 \mathrm{H}), 7.52(\mathrm{t}, J=$ $7.5 \mathrm{~Hz}, 4 \mathrm{H}), 7.28$ (t, $J=7.6 \mathrm{~Hz}, 1 \mathrm{H}), 7.17$ (d, $J=7.3 \mathrm{~Hz}, 1 \mathrm{H}), 7.09$ (t, $J=7.4 \mathrm{~Hz}, 1 \mathrm{H}), 7.04$ $(\mathrm{d}, J=8.1 \mathrm{~Hz}, 1 \mathrm{H}), 4.61-4.53(\mathrm{~m}, 1 \mathrm{H}), 3.73(\mathrm{dd}, J=18.5,8.1 \mathrm{~Hz}, 1 \mathrm{H}), 3.60(\mathrm{dd}, J=18.5$, $4.7 \mathrm{~Hz}, 1 \mathrm{H}), 3.33-3.27(\mathrm{~m}, 1 \mathrm{H}), 3.13-3.01(\mathrm{~m}, 2 \mathrm{H}) .{ }^{13} \mathbf{C}$ NMR (125 MHz, DMSO) $\delta$ 201.10, 197.71, 169.05, 150.84, 136.78, 136.02, 133.48, 133.18, 128.75, 128.70, 128.40, $128.38,128.15,127.96,124.31,122.86,116.01,40.63,40.19,39.02,26.45$. HRMS: $[\mathrm{M}+\mathrm{H}]^{+}$calcd. For $\mathrm{C}_{25} \mathrm{H}_{21} \mathrm{O}_{4} \mathrm{~m} / \mathrm{z}$ : 385.1440; found: $385.1442 .[\alpha]_{\mathbf{D}}{ }^{20}+13.5(c=1.0$ in $\mathrm{CHCl}_{3}$ ). The enantiomeric excess was determined by HPLC analysis on Daicel Chiralpak IC column ( $n$-hexane $/ i-\mathrm{PrOH}=70 / 30,1 \mathrm{~mL} / \mathrm{min}), \lambda=210 \mathrm{~nm}, t_{\text {major }}=13.24 \mathrm{~min}, t_{\text {minor }}=$ $16.97 \min , \boldsymbol{e} e \mathbf{= 9 4 . 2 \%}$.

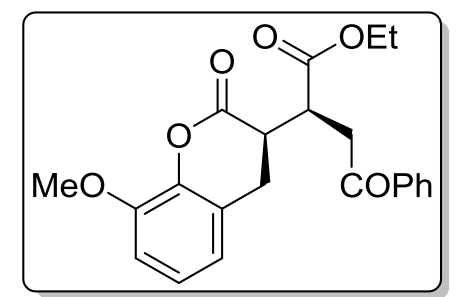

ethyl (S)-2-((R)-8-methoxy-2-oxochroman-3-yl)-4-oxo-4-phenylbutanoate (5q) 
$\mathbf{5 q}$ was obtained as a colorless oil $9 \mathrm{mg}$ in $\mathbf{2 3 \%}$ yield for two steps after column chromatography on silica gel with petroleum ether-ethyl acetate $\left(8: 1, \mathbf{R}_{f}=0.1\right)$ as eluant. ${ }^{1} \mathbf{H}$ NMR $\left(500 \mathrm{MHz}, \mathrm{CDCl}_{3}\right) \delta 7.98(\mathrm{t}, J=9.0 \mathrm{~Hz}, 2 \mathrm{H}), 7.58(\mathrm{t}, J=7.4 \mathrm{~Hz}, 1 \mathrm{H}), 7.47(\mathrm{t}, J=7.7$ $\mathrm{Hz}, 2 \mathrm{H}), 7.04(\mathrm{t}, J=7.9 \mathrm{~Hz}, 1 \mathrm{H}), 6.86(\mathrm{~d}, J=8.2 \mathrm{~Hz}, 1 \mathrm{H}), 6.75(\mathrm{~d}, J=7.5 \mathrm{~Hz}, 1 \mathrm{H}), 4.18(\mathrm{q}, J$ $=7.1 \mathrm{~Hz}, 2 \mathrm{H}), 3.89(\mathrm{~s}, 3 \mathrm{H}), 3.88-3.82(\mathrm{~m}, 1 \mathrm{H}), 3.65-3.60(\mathrm{~m}, 1 \mathrm{H}), 3.40-3.34(\mathrm{~m}, 1 \mathrm{H})$, $3.22(\mathrm{dd}, J=17.9,4.9 \mathrm{~Hz}, 1 \mathrm{H}), 3.01-2.90(\mathrm{~m}, 2 \mathrm{H}), 1.23(\mathrm{t}, J=7.1 \mathrm{~Hz}, 3 \mathrm{H}) .{ }^{13} \mathbf{C}$ NMR $(125$ $\left.\mathrm{MHz}, \mathrm{CDCl}_{3}\right) \delta 197.86,172.72,168.57,147.63,140.60,136.48,133.57,128.80,128.33$, $124.71,123.48,119.77,111.35,61.53,56.21,40.08,37.58,32.08,29.85,14.27$. HRMS: $[\mathrm{M}+\mathrm{H}]^{+}$calcd. For $\mathrm{C}_{22} \mathrm{H}_{23} \mathrm{O}_{6} \mathrm{~m} / \mathrm{z}$ : 383.1495; found: 383.1496. $[\boldsymbol{\alpha}]_{\mathbf{D}}{ }^{20}-2.4\left(c=0.21\right.$ in $\left.\mathrm{CHCl}_{3}\right)$. The enantiomeric excess was determined by HPLC analysis on Daicel Chiralpak IC column $(n$-hexane $/ i-\mathrm{PrOH}=70 / 30,1 \mathrm{~mL} / \mathrm{min}), \lambda=210 \mathrm{~nm}, t_{\text {major }}=47.29 \mathrm{~min}, t_{\text {minor }}=35.13 \mathrm{~min}, \boldsymbol{e} \boldsymbol{e}=$ $97.7 \%$.

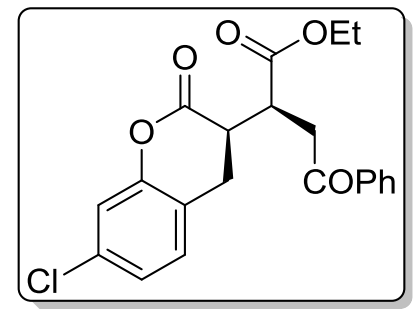

ethyl (S)-2-((R)-7-chloro-2-oxochroman-3-yl)-4-oxo-4-phenylbutanoate (5r)

$\mathbf{5 r}$ was obtained as a colorless oil $23 \mathrm{mg}$ in $\mathbf{5 9 \%}$ yield for two steps after column chromatography on silica gel with petroleum ether-ethyl acetate $\left(20: 1, \mathbf{R}_{f}=0.1\right)$ as eluant. ${ }^{1} \mathbf{H}$ $\operatorname{NMR}\left(500 \mathrm{MHz}, \mathrm{CDCl}_{3}\right) \delta 7.98(\mathrm{t}, J=7.7 \mathrm{~Hz}, 2 \mathrm{H}), 7.61-7.54(\mathrm{~m}, 1 \mathrm{H}), 7.51-7.43(\mathrm{~m}, 2 \mathrm{H})$, $7.15-7.03(\mathrm{~m}, 3 \mathrm{H}), 4.19(\mathrm{q}, J=7.1 \mathrm{~Hz}, 2 \mathrm{H}), 3.85(\mathrm{dd}, J=17.9,7.4 \mathrm{~Hz}, 1 \mathrm{H}), 3.70-3.62(\mathrm{~m}$, 1H), $3.45-3.34(\mathrm{~m}, 1 \mathrm{H}), 3.19(\mathrm{dd}, J=17.9,5.4 \mathrm{~Hz}, 1 \mathrm{H}), 3.03-2.90(\mathrm{~m}, 2 \mathrm{H}), 1.23(\mathrm{t}, J=7.5$ $\mathrm{Hz}, 3 \mathrm{H}) .{ }^{13} \mathrm{C}$ NMR $\left(125 \mathrm{MHz}, \mathrm{CDCl}_{3}\right) \delta 197.58,172.53,168.50,151.72,136.38,133.88$, 133.66, 133.64, 129.21, 128.84, 128.82, 128.30, 128.23, 124.85, 121.38, 61.61, 40.70, 39.98, 37.37, 27.27, 14.20. HRMS: $[\mathrm{M}+\mathrm{H}]^{+}$calcd. For $\mathrm{C}_{21} \mathrm{H}_{20} \mathrm{ClO}_{5} \mathrm{~m} / \mathrm{z}$ : 387.0999; found: 387.0998. $[\boldsymbol{\alpha}]_{\mathbf{D}}{ }^{\mathbf{2 0}}+12.2\left(c=1.08\right.$ in $\left.\mathrm{CHCl}_{3}\right)$. The enantiomeric excess was determined by HPLC analysis on Daicel Chiralpak IB column ( $n$-hexane $/ i$-PrOH $=90 / 10,1 \mathrm{~mL} / \mathrm{min}), \lambda=210 \mathrm{~nm}, t_{\text {major }}=$ $16.29 \mathrm{~min}, t_{\text {minor }}=14.87 \mathrm{~min}, \boldsymbol{e} e=\mathbf{9 5 . 9 \%}$. 


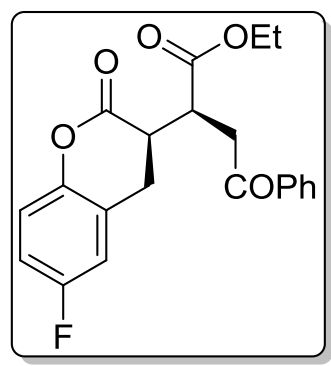

ethyl (S)-2-((R)-6-fluoro-2-oxochroman-3-yl)-4-oxo-4-phenylbutanoate (5s)

5s was obtained as a colorless oil $20 \mathrm{mg}$ in $\mathbf{5 4 \%}$ yield for two steps after column chromatography on silica gel with petroleum ether-ethyl acetate $\left(20: 1, \mathbf{R}_{f}=0.1\right)$ as eluant. ${ }^{1} \mathbf{H}$ NMR $\left(500 \mathrm{MHz}, \mathrm{CDCl}_{3}\right) \delta 7.99(\mathrm{~d}, J=7.4 \mathrm{~Hz}, 2 \mathrm{H}), 7.59(\mathrm{t}, J=7.4 \mathrm{~Hz}, 1 \mathrm{H}), 7.48(\mathrm{t}, J=7.7$ $\mathrm{Hz}, 2 \mathrm{H}), 7.02(\mathrm{dd}, J=8.9,4.6 \mathrm{~Hz}, 1 \mathrm{H}), 6.99-6.93(\mathrm{~m}, 1 \mathrm{H}), 6.91-6.86(\mathrm{~m}, 1 \mathrm{H}), 4.19(\mathrm{q}, J=$ $7.1 \mathrm{~Hz}, 2 \mathrm{H}), 3.85(\mathrm{dd}, J=17.9,7.4 \mathrm{~Hz}, 1 \mathrm{H}), 3.68-3.61(\mathrm{~m}, 1 \mathrm{H}), 3.41-3.33(\mathrm{~m}, 1 \mathrm{H}), 3.20$ $(\mathrm{dd}, J=17.9,5.4 \mathrm{~Hz}, 1 \mathrm{H}), 3.06-2.90(\mathrm{~m}, 2 \mathrm{H}), 1.24(\mathrm{t}, J=7.1 \mathrm{~Hz}, 3 \mathrm{H}) .{ }^{13} \mathbf{C} \mathbf{N M R}(125 \mathrm{MHz}$, $\left.\mathrm{CDCl}_{3}\right) \delta 197.61,172.59,168.82,(160.15,158.21),(147.40, \quad 147.38), 136.37,133.61$, 128.84, 128.31, (124.00, 123.94), (115.40, 115.21), (115.06, 114.87), 61.53, 40.45, 40.04, 37.27, 27.70, 14.21. The Carbon spectral was splitted by fluorine . HRMS: $[\mathrm{M}+\mathrm{H}]^{+}$calcd. For $\mathrm{C}_{21} \mathrm{H}_{20} \mathrm{FO}_{5} \mathrm{~m} / \mathrm{z}: 371.1295$; found: 371.1297. $[\boldsymbol{\alpha}]_{\mathbf{D}}{ }^{20}+10.4\left(c=1.25\right.$ in $\left.\mathrm{CHCl}_{3}\right)$. The enantiomeric excess was determined by HPLC analysis on Daicel Chiralpak IC column $(n$-hexane $/ i-\mathrm{PrOH}=70 / 30,1 \mathrm{~mL} / \mathrm{min}), \lambda=210 \mathrm{~nm}, t_{\text {major }}=21.27 \mathrm{~min}, t_{\text {minor }}=17.94 \mathrm{~min}, \boldsymbol{e} \boldsymbol{e}=$ $98.2 \%$.

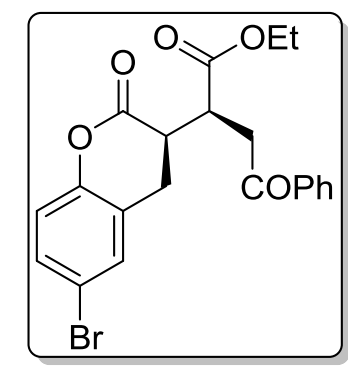

ethyl (S)-2-((R)-6-bromo-2-oxochroman-3-yl)-4-oxo-4-phenylbutanoate (5t)

5t was obtained as a colorless oil $23 \mathrm{mg}$ in 53\% yield for two steps after column chromatography on silica gel with petroleum ether-ethyl acetate $\left(20: 1, \mathbf{R}_{f}=0.1\right)$ as eluant. ${ }^{1} \mathbf{H}$ NMR $\left(500 \mathrm{MHz}, \mathrm{CDCl}_{3}\right) \delta 7.98(\mathrm{t}, J=6.9 \mathrm{~Hz}, 2 \mathrm{H}), 7.59(\mathrm{t}, J=7.4 \mathrm{~Hz}, 1 \mathrm{H}), 7.48(\mathrm{t}, J=7.7$ $\mathrm{Hz}, 2 \mathrm{H}), 7.40-7.30(\mathrm{~m}, 2 \mathrm{H}), 6.93(\mathrm{t}, J=8.4 \mathrm{~Hz}, 1 \mathrm{H}), 4.19(\mathrm{q}, J=7.1 \mathrm{~Hz}, 2 \mathrm{H}), 3.83(\mathrm{dd}, J=$ $17.9,7.3 \mathrm{~Hz}, 1 \mathrm{H}), 3.69-3.61(\mathrm{~m}, 1 \mathrm{H}), 3.40-3.31(\mathrm{~m}, 1 \mathrm{H}), 3.20(\mathrm{dd}, J=17.9,5.5 \mathrm{~Hz}, 1 \mathrm{H})$, 
$3.06-2.89(\mathrm{~m}, 2 \mathrm{H}), 1.24(\mathrm{t}, J=7.1 \mathrm{~Hz}, 3 \mathrm{H}) .{ }^{13} \mathbf{C} \mathbf{~ N M R}\left(125 \mathrm{MHz}, \mathrm{CDCl}_{3}\right) \delta 197.53,172.52$, $168.55,150.44,136.37,133.67,131.61,131.15,128.84,128.30,124.41,118.54,117.25$, 61.63, 40.52, 39.95, 37.40, 27.44, 14.20. HRMS: $[\mathrm{M}+\mathrm{H}]^{+}$calcd. For $\mathrm{C}_{21} \mathrm{H}_{20} \mathrm{BrO}_{5} \mathrm{~m} / \mathrm{z}$ : 431.0494; found: 431.0497. $[\alpha]_{\mathbf{D}}{ }^{20}-6.2\left(c=0.83\right.$ in $\left.\mathrm{CHCl}_{3}\right)$. The enantiomeric excess was determined by HPLC analysis on Daicel Chiralpak IC column ( $n$-hexane/i-PrOH $=70 / 30,1$ $\mathrm{mL} / \mathrm{min}), \lambda=210 \mathrm{~nm}, t_{\text {major }}=22.27 \mathrm{~min}, t_{\text {minor }}=18.73 \mathrm{~min}, \boldsymbol{e} \boldsymbol{e}=\mathbf{9 7 . 9 \%}$.

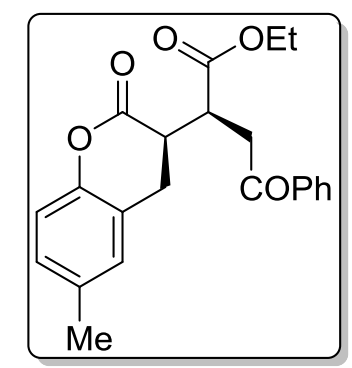

ethyl (S)-2-((R)-6-methyl-2-oxochroman-3-yl)-4-oxo-4-phenylbutanoate (5u)

5u was obtained as a colorless oil $14 \mathrm{mg}$ in $\mathbf{4 7 \%}$ yield for two steps after column chromatography on silica gel with petroleum ether-ethyl acetate $\left(25: 1, \mathrm{R}_{f}=0.1\right)$ as eluant. ${ }^{1} \mathbf{H}$ NMR $\left(500 \mathrm{MHz}, \mathrm{CDCl}_{3}\right) \delta 7.99(\mathrm{t}, J=8.0 \mathrm{~Hz}, 2 \mathrm{H}), 7.58(\mathrm{t}, J=7.4 \mathrm{~Hz}, 1 \mathrm{H}), 7.47(\mathrm{t}, J=7.7$ $\mathrm{Hz}, 2 \mathrm{H}), 7.05(\mathrm{~d}, J=8.2 \mathrm{~Hz}, 1 \mathrm{H}), 6.99-6.91(\mathrm{~m}, 2 \mathrm{H}), 4.19(\mathrm{q}, J=7.1 \mathrm{~Hz}, 2 \mathrm{H}), 3.85(\mathrm{dd}, J=$ 17.9, 7.8 Hz, 1H), $3.67-3.59(\mathrm{~m}, 1 \mathrm{H}), 3.40-3.31(\mathrm{~m}, 1 \mathrm{H}), 3.20$ (dd, $J=17.9,5.0 \mathrm{~Hz}, 1 \mathrm{H})$, $3.01-2.86(\mathrm{~m}, 2 \mathrm{H}), 2.31(\mathrm{~s}, 3 \mathrm{H}), 1.24(\mathrm{t}, J=7.2 \mathrm{~Hz}, 3 \mathrm{H}) .{ }^{13} \mathbf{C} \mathbf{N M R}\left(125 \mathrm{MHz}, \mathrm{CDCl}_{3}\right) \delta$ $197.80,172.76,169.44,149.29,136.50,134.36,133.56,129.07,128.80,128.75,128.32$, 121.95, 116.50, 61.50, 41.07, 40.15, 37.50, 27.79, 20.86, 14.21. HRMS: $[\mathrm{M}+\mathrm{H}]^{+}$calcd. For $\mathrm{C}_{22} \mathrm{H}_{23} \mathrm{O}_{5} \mathrm{~m} / \mathrm{z}: 367.1545$; found: 367.1542 . $[\alpha]_{\mathbf{D}}{ }^{20}+2.9\left(c=0.75\right.$ in $\left.\mathrm{CHCl}_{3}\right)$. The enantiomeric excess was determined by HPLC analysis on Daicel Chiralpak IC column ( $n$-hexane/i-PrOH $=70 / 30,1 \mathrm{~mL} / \mathrm{min}), \lambda=210 \mathrm{~nm}, t_{\text {major }}=23.47 \mathrm{~min}, t_{\text {minor }}=20.77 \mathrm{~min}, \boldsymbol{e} \boldsymbol{e}=\mathbf{9 8 . 0 \%}$.

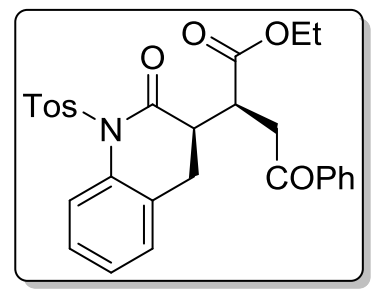

ethyl (S)-4-oxo-2-((R)-2-oxo-1-tosyl-1,2,3,4-tetrahydroquinolin-3-yl)-4-phenylbutanoate (5v)

5v was obtained as a colorless oil $28.8 \mathrm{mg}$ in $\mathbf{5 7 \%}$ yield for two steps after column 
chromatography on silica gel with petroleum ether-ethyl acetate $\left(20: 3, R_{f}=0.1\right)$ as eluant. ${ }^{1} \mathbf{H}$ NMR $\left(500 \mathrm{MHz}, \mathrm{CDCl}_{3}\right) \delta 7.93(\mathrm{dd}, J=16.9,8.2 \mathrm{~Hz}, 2 \mathrm{H}), 7.85(\mathrm{t}, J=7.2 \mathrm{~Hz}, 2 \mathrm{H})$, $7.64(\mathrm{t}, J=7.6 \mathrm{~Hz}, 1 \mathrm{H}), 7.57(\mathrm{t}, J=7.3 \mathrm{~Hz}, 1 \mathrm{H}), 7.45(\mathrm{t}, J=7.6 \mathrm{~Hz}, 2 \mathrm{H}), 7.38-7.28(\mathrm{~m}$, 3H), $7.25-7.19(\mathrm{~m}, 2 \mathrm{H}), 4.12-4.04(\mathrm{~m}, 2 \mathrm{H}), 3.55(\mathrm{dd}, J=18.0,7.7 \mathrm{~Hz}, 1 \mathrm{H}), 3.43-3.32$ (m, 1H), $3.16-3.09(\mathrm{~m}, 1 \mathrm{H}), 2.78(\mathrm{dd}, J=18.2,4.8 \mathrm{~Hz}, 1 \mathrm{H}), 2.72(\mathrm{~d}, J=8.4 \mathrm{~Hz}, 2 \mathrm{H}), 2.41$ (s, 3H), $1.16(\mathrm{t}, J=7.1 \mathrm{~Hz}, 3 \mathrm{H}) .{ }^{13} \mathbf{C}$ NMR $\left(125 \mathrm{MHz}, \mathrm{CDCl}_{3}\right) \delta 197.79,172.66,171.76$, $145.13,136.81,136.48,135.14,133.46,129.54,129.33,128.87,128.70,128.22,127.81$, $127.53,126.72,124.02,61.38,39.96,37.10,29.84,29.35,21.84,14.16$. HRMS: $[\mathrm{M}+\mathrm{H}]^{+}$

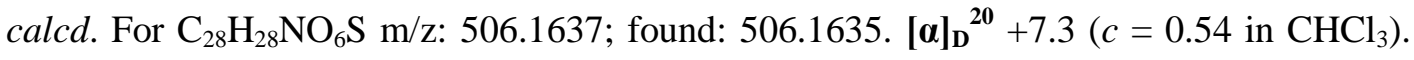
The enantiomeric excess was determined by HPLC analysis on Daicel Chiralpak IC column $(n$-hexane $/ i-\mathrm{PrOH}=80 / 20,1 \mathrm{~mL} / \mathrm{min}), \lambda=210 \mathrm{~nm}, t_{\text {major }}=23.15 \mathrm{~min}, t_{\text {minor }}=21.63$ $\min , \boldsymbol{e} \boldsymbol{e}=\mathbf{9 1 . 6 \%}$. 


\section{Five-step in one pot}

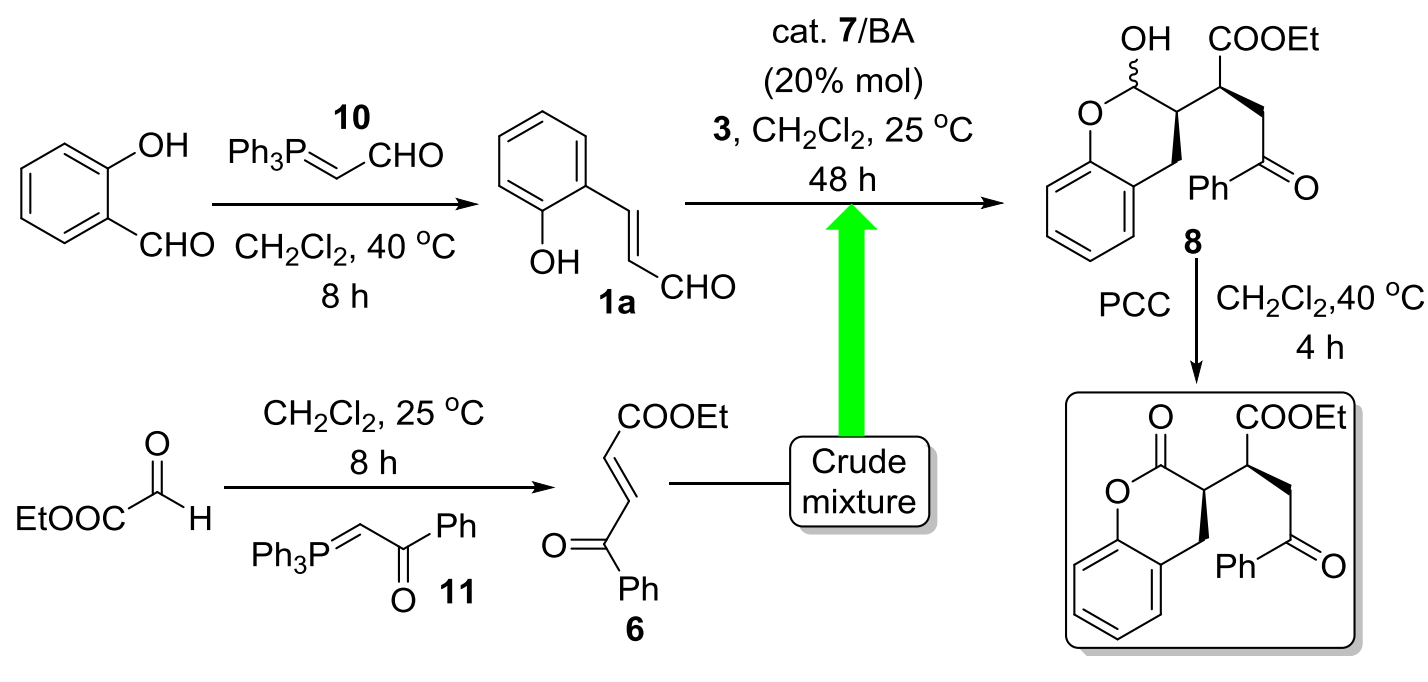

$5,37 \%, 98.9 \%$ ee

Procedure: A solution of $10(0.11 \mathrm{mmol}, 33.5 \mathrm{mg})$ in $\mathrm{CH}_{2} \mathrm{Cl}_{2}(0.1 \mathrm{~mL})$ was added salicylaldehyde $(0.1 \mathrm{mmol}, 11 \mu \mathrm{L})$ at $40{ }^{\circ} \mathrm{C}$ for $8 \mathrm{~h}$ in bottle $\mathbf{I}$. In the meantime, a solution of $10(0.11 \mathrm{mmol}, 41.8 \mathrm{mg})$ in $\mathrm{CH}_{2} \mathrm{Cl}_{2}(0.1 \mathrm{~mL})$ was added Ethyl glyoxalate $(0.11 \mathrm{mmol}, 11 \mu \mathrm{L})$ at $25{ }^{\circ} \mathrm{C}$ for $8 \mathrm{~h}$ in bottle II. After the completion of the material of salicylaldehyde and $\mathbf{1 1}$, the crude mixture of bottle II, cat $7(0.02 \mathrm{mmol}, 6.5 \mathrm{mg})$ in $\mathrm{CH}_{2} \mathrm{Cl}_{2}(0.1 \mathrm{~mL}), \mathrm{BA}(0.02 \mathrm{mmol}$, $6.5 \mathrm{mg}), \mathbf{3}(0.11 \mathrm{mmol}, 27.8 \mathrm{mg})$ were all addde to the bottle $\mathbf{I}$ for another $48 \mathrm{~h}$ at $25^{\circ} \mathrm{C}$.Then $\mathrm{CH}_{2} \mathrm{Cl}_{2}(0.8 \mathrm{~mL})$ and PCC (Pyridinium Chlorochromate $0.36 \mathrm{mmol}, 3.6$ equiv) with $78 \mathrm{mg}$ celite were added to bottle I for a further $4 \mathrm{~h}$ at $40{ }^{\circ} \mathrm{C}$. The reaction mixture was filtrated through a short plug of silica gel. Solvent was removed under reduced pressure and the crude product was purified by column chromatography on silica gel (petroleum ether / ethyl acetate $\left.=15: 1, \mathbf{R}_{f}=0.1\right)$ to provide the desired product $\mathbf{5}$ as a coluorless oil $(13 \mathrm{mg}, 37 \%$ yield, $\boldsymbol{e} \boldsymbol{e}=$ $98.9 \%)$. 


\section{Synthetic transformation}

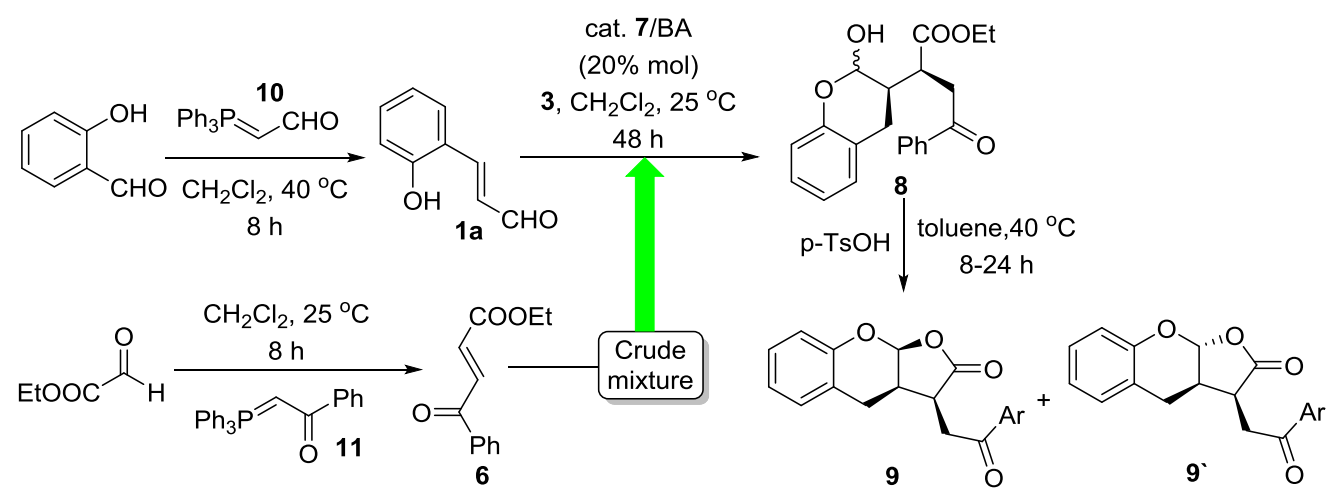

General Procedure: A solution of $\mathbf{1 0}(0.11 \mathrm{mmol}, 33.5 \mathrm{mg})$ in $\mathrm{CH}_{2} \mathrm{Cl}_{2}(0.1 \mathrm{~mL})$ was added salicylaldehyde $(0.1 \mathrm{mmol}, 11 \mu \mathrm{L})$ at $40{ }^{\circ} \mathrm{C}$ for $8 \mathrm{~h}$ in bottle $\mathbf{I}$. In the meantime, a solution of $11(0.11 \mathrm{mmol}, 41.8 \mathrm{mg})$ in $\mathrm{CH}_{2} \mathrm{Cl}_{2}(0.1 \mathrm{~mL})$ was added Ethyl glyoxalate $(0.11 \mathrm{mmol}, 11 \mu \mathrm{L})$ at $25{ }^{\circ} \mathrm{C}$ for $8 \mathrm{~h}$ in bottle II. After the completion of the material of salicylaldehyde and $\mathbf{1 1}$, the crude mixture of bottle II, cat $7(0.02 \mathrm{mmol}, 6.5 \mathrm{mg})$ in $\mathrm{CH}_{2} \mathrm{Cl}_{2}(0.1 \mathrm{~mL})$, BA $(0.02 \mathrm{mmol}$, $6.5 \mathrm{mg}), \mathbf{3}(0.11 \mathrm{mmol}, 27.8 \mathrm{mg})$ were all addde to the bottle $\mathbf{I}$ for another $48 \mathrm{~h}$ at $25^{\circ} \mathrm{C}$. A solution of $8(0.1 \mathrm{mmol})$, the equivalent $4 \AA$ molecular sieve, $p$-TsOH $(0.02 \mathrm{mmol})$ in toluene $(1 \mathrm{~mL})$ was heated to $40{ }^{\circ} \mathrm{C}$. After the completion of the reaction indicated by TLC, the reaction mixture was cooled to the room temperature. The residue was purified by column chromatography on silica gel to afford the product 9 and 9 '.

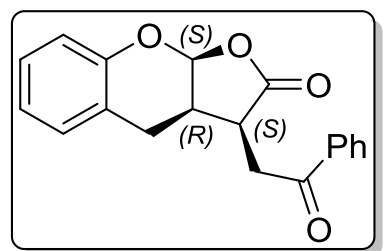

(3S,3aR,9aR)-3-(2-oxo-2-phenylethyl)-3,3a,4,9a-tetrahydro-2Hfuro[2,3-b]chromen-2-one (9a)

9a was obtained as a white solid $15 \mathrm{mg}$ in $\mathbf{4 9 \%}$ yield after column chromatography on silica gel with petroleum ether-ethyl acetate $\left(10: 1, \mathbf{R}_{f}=0.1\right)$ as eluant. ${ }^{1} \mathbf{H} \mathbf{N M R}\left(500 \mathrm{MHz}, \mathrm{CDCl}_{3}\right)$ $\delta 7.97-7.93(\mathrm{~m}, 2 \mathrm{H}), 7.63-7.58(\mathrm{~m}, 1 \mathrm{H}), 7.48(\mathrm{t}, J=7.8 \mathrm{~Hz}, 2 \mathrm{H}), 7.21(\mathrm{t}, J=7.8 \mathrm{~Hz}, 1 \mathrm{H})$, $7.18(\mathrm{~d}, J=7.3 \mathrm{~Hz}, 1 \mathrm{H}), 7.06-7.01(\mathrm{~m}, 1 \mathrm{H}), 7.00(\mathrm{~d}, J=8.0 \mathrm{~Hz}, 1 \mathrm{H}), 6.31(\mathrm{~d}, J=7.1 \mathrm{~Hz}$, 1H), $3.55(\mathrm{dd}, J=18.4,3.2 \mathrm{~Hz}, 1 \mathrm{H}), 3.37(\mathrm{dd}, J=18.4,6.9 \mathrm{~Hz}, 1 \mathrm{H}), 3.16-3.09(\mathrm{~m}, 1 \mathrm{H})$, 
$2.99(\mathrm{dd}, J=15.4,5.2 \mathrm{~Hz}, 1 \mathrm{H}), 2.84-2.75(\mathrm{~m}, 2 \mathrm{H}) .{ }^{13} \mathbf{C} \mathbf{N M R}\left(125 \mathrm{MHz}, \mathrm{CDCl}_{3}\right) \delta 196.66$, $177.35,152.47,136.06,133.95,129.57,128.96,128.68,128.22,123.73,123.59,118.51$, 100.28, 40.58, 38.60, 37.80, 26.06. HRMS: $[\mathrm{M}+\mathrm{H}]^{+}$calcd. For $\mathrm{C}_{19} \mathrm{H}_{17} \mathrm{O}_{4} \mathrm{~m} / \mathrm{z}: 309.1127$; found: 309.1129. $[\boldsymbol{\alpha}]_{\mathbf{D}}{ }^{20}+48.9\left(c=0.37\right.$ in $\left.\mathrm{CHCl}_{3}\right)$. The diastereomeric ratio was determined by HPLC analysis, $\boldsymbol{d} \boldsymbol{r}=\mathbf{5 . 3 : 1}$. The enantiomeric excess was determined by HPLC using Chiralpak IB column $(n$-hexane $/ i-\mathrm{PrOH}=80 / 20,1 \mathrm{~mL} / \mathrm{min}), \lambda=210 \mathrm{~nm}, t_{\text {major }}=18.75 \mathrm{~min}$, $t_{\text {minor }}=15.89 \min , \boldsymbol{e e}=\mathbf{9 9 . 9 \%}$.

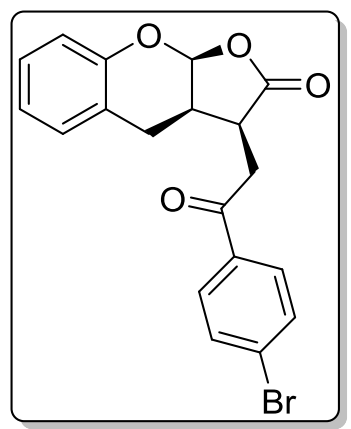

(3S,3aR,9aS)-3-(2-(4-bromophenyl)-2-oxoethyl)-3,3a,4,9a-tetrahydro-2 $\mathrm{H}$ furo[2,3-b]chromen-2-one (9b)

9b was obtained as a white solid $16.8 \mathrm{mg}$ in $\mathbf{4 3 \%}$ yield after column chromatography on silica gel with petroleum ether-ethyl acetate $\left(10: 1, \mathbf{R}_{f}=0.1\right)$ as eluant. ${ }^{1} \mathbf{H} \mathbf{N M R}(500 \mathrm{MHz}$, $\left.\mathrm{CDCl}_{3}\right) \delta 7.85(\mathrm{~d}, J=8.5 \mathrm{~Hz}, 2 \mathrm{H}), 7.66(\mathrm{~d}, J=8.5 \mathrm{~Hz}, 2 \mathrm{H}), 7.23-7.17(\mathrm{~m}, 1 \mathrm{H}), 6.99(\mathrm{~d}, J=$ $8.1 \mathrm{~Hz}, 1 \mathrm{H}), 6.97-6.92(\mathrm{~m}, 2 \mathrm{H}), 6.26(\mathrm{~d}, J=5.1 \mathrm{~Hz}, 1 \mathrm{H}), 3.64-3.53(\mathrm{~m}, 2 \mathrm{H}), 3.32-3.25$ (m, 1H), $3.20(\mathrm{dd}, J=18.9,10.7 \mathrm{~Hz}, 1 \mathrm{H}), 2.67(\mathrm{dd}, J=15.6,6.7 \mathrm{~Hz}, 1 \mathrm{H}), 2.41(\mathrm{dd}, J=15.6$, $8.5 \mathrm{~Hz}, 1 \mathrm{H}) .{ }^{13} \mathbf{C} \mathbf{N M R}\left(125 \mathrm{MHz}, \mathrm{CDCl}_{3}\right) \delta 195.96,175.02,151.32,134.66,132.39,129.74$, $129.37,128.82,128.45,123.22,121.43,117.75,99.96,40.67,36.15,34.84,29.85$. HRMS: $[\mathrm{M}+\mathrm{H}]^{+}$calcd. For $\mathrm{C}_{19} \mathrm{H}_{16} \mathrm{BrO}_{4} \mathrm{~m} / \mathrm{z}$ : 387.0232; found: 387.0233. $[\boldsymbol{\alpha}]_{\mathbf{D}}{ }^{20}+50.2(c=0.42$ in $\mathrm{CHCl}_{3}$ ). The diastereomeric ratio was determined by HPLC analysis, $\boldsymbol{d} \boldsymbol{r}=\mathbf{6 : 1}$. The enantiomeric excess was determined by HPLC using Chiralpak IB column ( $n$-hexane/i-PrOH $=80 / 20,1 \mathrm{~mL} / \mathrm{min}), \lambda=210 \mathrm{~nm}, t_{\text {major }}=24.01 \mathrm{~min}, t_{\text {minor }}=19.81 \mathrm{~min}, \boldsymbol{e} \boldsymbol{e}=\mathbf{9 8 . 6 \%}$. 


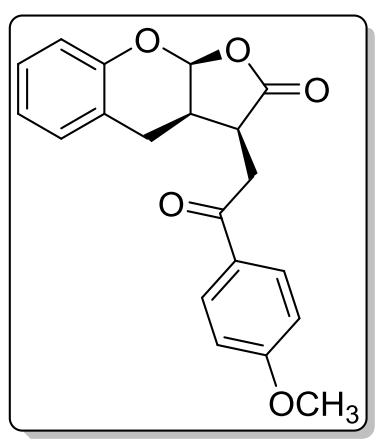

(3S,3aR,9aR)-3-(2-(4-methoxyphenyl)-2-oxoethyl)-3,3a,4,9a-tetrahydro$2 \mathrm{H}$-furo[2,3-b]chromen-2-one (9c)

9c was obtained as a white solid $11 \mathrm{mg}$ in $\mathbf{3 3 \%}$ yield after column chromatography on silica gel with petroleum ether-ethyl acetate $\left(5: 1, \mathrm{R}_{f}=0.1\right)$ as eluant. ${ }^{1} \mathbf{H} \mathbf{~ N M R}\left(500 \mathrm{MHz}, \mathrm{CDCl}_{3}\right)$ $\delta 7.92(\mathrm{~d}, J=8.7 \mathrm{~Hz}, 2 \mathrm{H}), 7.24-7.15(\mathrm{~m}, 2 \mathrm{H}), 7.06-6.97(\mathrm{~m}, 2 \mathrm{H}), 6.94(\mathrm{~d}, J=8.7 \mathrm{~Hz}, 2 \mathrm{H})$, $6.30(\mathrm{~d}, J=7.1 \mathrm{~Hz}, 1 \mathrm{H}), 3.88(\mathrm{~s}, 3 \mathrm{H}), 3.49(\mathrm{dd}, J=18.2,2.9 \mathrm{~Hz}, 1 \mathrm{H}), 3.32(\mathrm{dd}, J=18.2,6.9$ Hz, 1H), $3.17-3.08(\mathrm{~m}, 1 \mathrm{H}), 2.97(\mathrm{dd}, J=15.4,5.0 \mathrm{~Hz}, 1 \mathrm{H}), 2.81(\mathrm{~d}, J=15.0 \mathrm{~Hz}, 1 \mathrm{H}), 2.78$ - $2.72(\mathrm{~m}, 1 \mathrm{H}) .{ }^{13} \mathrm{C}$ NMR $\left(125 \mathrm{MHz}, \mathrm{CDCl}_{3}\right) \delta 195.07,177.50,164.12,152.47,130.52$, $129.55,129.17,128.62,123.85,123.55,118.48,114.07,100.31,55.68,40.56,38.19,37.92$,

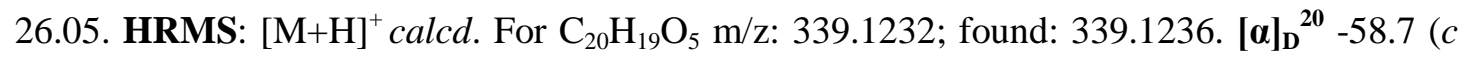
$=0.83$ in $\left.\mathrm{CHCl}_{3}\right)$. The diastereomeric ratio was determined by HPLC analysis, $\boldsymbol{d} \boldsymbol{r}=\mathbf{4 : 1}$. The enantiomeric excess was determined by HPLC using Chiralpak IB column ( $n$-hexane/i-PrOH $=80 / 20,1 \mathrm{~mL} / \mathrm{min}), \lambda=210 \mathrm{~nm}, t_{\text {major }}=27.60 \mathrm{~min}, t_{\text {minor }}=25.51 \mathrm{~min}, \boldsymbol{e} \boldsymbol{e}=\mathbf{9 7 . 1 \%}$.

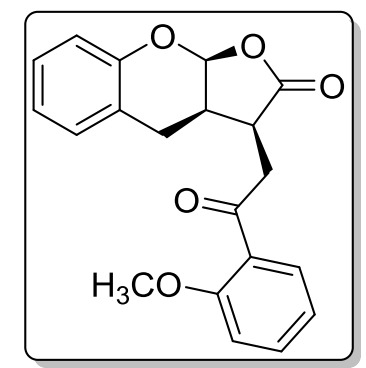

(3S,3aR,9aS)-3-(2-(2-methoxyphenyl)-2-oxoethyl)-3,3a,4,9a-tetrahydro-2 $H$ furo[2,3-b]chromen-2-one (9d)

9d was obtained as a white solid $14 \mathrm{mg}$ in $\mathbf{4 2 \%}$ yield after column chromatography on silica gel with petroleum ether-ethyl acetate $\left(5: 1, \mathrm{R}_{f}=0.1\right)$ as eluant. ${ }^{1} \mathbf{H} \mathbf{~ N M R}\left(500 \mathrm{MHz}, \mathrm{CDCl}_{3}\right)$ $\delta 7.81(\mathrm{dd}, J=7.7,1.7 \mathrm{~Hz}, 1 \mathrm{H}), 7.57-7.50(\mathrm{~m}, 1 \mathrm{H}), 7.22-7.16(\mathrm{~m}, 1 \mathrm{H}), 7.08-6.90(\mathrm{~m}$, $5 \mathrm{H}), 6.22(\mathrm{~d}, J=5.1 \mathrm{~Hz}, 1 \mathrm{H}), 3.94(\mathrm{~s}, 3 \mathrm{H}), 3.65-3.56(\mathrm{~m}, 2 \mathrm{H}), 3.37-3.28(\mathrm{~m}, 1 \mathrm{H}), 3.28-$ $3.19(\mathrm{~m}, 1 \mathrm{H}), 2.69(\mathrm{dd}, J=15.7,6.7 \mathrm{~Hz}, 1 \mathrm{H}), 2.44(\mathrm{dd}, J=15.7,8.8 \mathrm{~Hz}, 1 \mathrm{H}) .{ }^{13} \mathbf{C}$ NMR $(125$ 
$\left.\mathrm{MHz}, \mathrm{CDCl}_{3}\right) \delta 198.63,175.30,159.30,151.41,134.63,130.76,128.60,128.58,126.75$, 122.96, 121.75, 120.98, 117.60, 111.88, 99.88, 55.75, 41.06, 39.89, 36.30, 22.82. HRMS: $[\mathrm{M}+\mathrm{H}]^{+}$calcd. For $\mathrm{C}_{20} \mathrm{H}_{19} \mathrm{O}_{5} \mathrm{~m} / \mathrm{z}$ : 339.1232; found: 339.1235. $[\boldsymbol{\alpha}]_{\mathbf{D}}{ }^{20}+21.2(c=0.45$ in $\mathrm{CHCl}_{3}$ ). The diastereomeric ratio was determined by HPLC analysis, $\boldsymbol{d} \boldsymbol{r}=\mathbf{3 : 1}$. The enantiomeric excess was determined by HPLC using Chiralpak IB column ( $n$-hexane/i-PrOH $=80 / 20,1 \mathrm{~mL} / \mathrm{min}), \lambda=210 \mathrm{~nm}, t_{\text {major }}=21.93 \mathrm{~min}, t_{\text {minor }}=14.84 \mathrm{~min}, \boldsymbol{e} \boldsymbol{e}=\mathbf{9 8 . 0 \%}$.

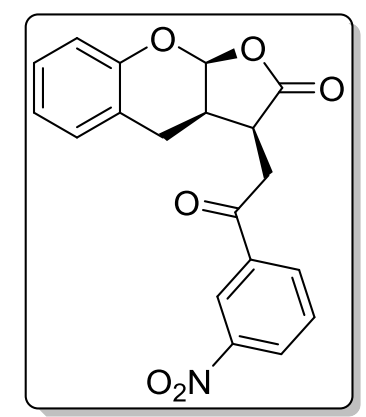

(3S,3aR,9aS)-3-(2-(3-nitrophenyl)-2-oxoethyl)-3,3a,4,9a-tetrahydro$2 H$-furo[2,3-b]chromen-2-one (9e)

9e was obtained as a white solid $12 \mathrm{mg}$ in $\mathbf{3 4 \%}$ yield after column chromatography on silica gel with petroleum ether-ethyl acetate $\left(5: 1, \mathrm{R}_{f}=0.1\right)$ as eluant. ${ }^{1} \mathbf{H} \mathbf{~ N M R}\left(500 \mathrm{MHz}, \mathrm{CDCl}_{3}\right)$ $\delta 8.80(\mathrm{t}, J=1.6 \mathrm{~Hz}, 1 \mathrm{H}), 8.51-8.46(\mathrm{~m}, 1 \mathrm{H}), 8.30(\mathrm{~d}, J=7.8 \mathrm{~Hz}, 1 \mathrm{H}), 7.74(\mathrm{t}, J=8.0 \mathrm{~Hz}$, 1H), $7.24-7.18(\mathrm{~m}, 1 \mathrm{H}), 7.00(\mathrm{~d}, J=8.2 \mathrm{~Hz}, 1 \mathrm{H}), 6.98-6.92(\mathrm{~m}, 2 \mathrm{H}), 6.27(\mathrm{~d}, J=5.0 \mathrm{~Hz}$, $1 \mathrm{H}), 3.70-3.62(\mathrm{~m}, 2 \mathrm{H}), 3.33-3.21(\mathrm{~m}, 2 \mathrm{H}), 2.71(\mathrm{dd}, J=15.6,6.6 \mathrm{~Hz}, 1 \mathrm{H}), 2.45(\mathrm{dd}, J=$ 15.6, $8.7 \mathrm{~Hz}, 1 \mathrm{H}) .{ }^{13} \mathbf{C}$ NMR $\left(125 \mathrm{MHz}, \mathrm{CDCl}_{3}\right) \delta 194.87,174.74,151.29,148.75,137.18$, $133.71,130.37,128.92,128.47,128.20,123.23,123.16,121.03,117.73,99.88,40.66,36.09$, 35.16, 22.86. HRMS: $[\mathrm{M}+\mathrm{H}]^{+}$calcd. For $\mathrm{C}_{19} \mathrm{H}_{16} \mathrm{NO}_{6} \mathrm{~m} / \mathrm{z}$ : 354.0978; found: 354.0979. $[\boldsymbol{\alpha}]_{\mathbf{D}}{ }^{20}$ $+42.9\left(c=0.64\right.$ in $\left.\mathrm{CHCl}_{3}\right)$. The diastereomeric ratio was determined by HPLC analysis, $\boldsymbol{d} \boldsymbol{r}=$ 3:1. The enantiomeric excess was determined by HPLC using Chiralpak IB column $(n$-hexane $/ i-\mathrm{PrOH}=70 / 30,1 \mathrm{~mL} / \mathrm{min}), \lambda=210 \mathrm{~nm}, t_{\text {major }}=32.71 \mathrm{~min}, t_{\text {minor }}=28.64 \mathrm{~min}, \boldsymbol{e e}=$ $99.4 \%$. 


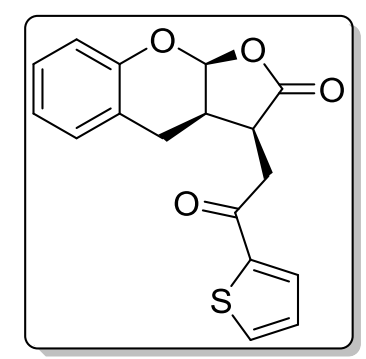

(3S,3aR,9aS)-3-(2-oxo-2-(thiophen-2-yl)ethyl)-3,3a,4,9a-tetrahydro-

$2 H$-furo[2,3-b]chromen-2-one (9f)

9f was obtained as a white solid $7.7 \mathrm{mg}$ in $\mathbf{2 4 \%}$ yield after column chromatography on silica gel with petroleum ether-ethyl acetate $\left(6: 1, \mathrm{R}_{f}=0.1\right)$ as eluant. ${ }^{1} \mathbf{H} \mathbf{~ N M R}\left(500 \mathrm{MHz}, \mathrm{CDCl}_{3}\right)$ $\delta 7.77(\mathrm{~d}, J=3.2 \mathrm{~Hz}, 1 \mathrm{H}), 7.72(\mathrm{~d}, J=4.4 \mathrm{~Hz}, 1 \mathrm{H}), 7.23-7.16(\mathrm{~m}, 2 \mathrm{H}), 7.03-6.92(\mathrm{~m}, 3 \mathrm{H})$, $6.24(\mathrm{~d}, J=5.1 \mathrm{~Hz}, 1 \mathrm{H}), 3.64-3.51(\mathrm{~m}, 2 \mathrm{H}), 3.32-3.24(\mathrm{~m}, 1 \mathrm{H}), 3.20(\mathrm{dd}, J=18.0,10.5 \mathrm{~Hz}$ 1H), $2.72(\mathrm{dd}, J=15.7,6.6 \mathrm{~Hz}, 1 \mathrm{H}), 2.45(\mathrm{dd}, J=15.6,8.6 \mathrm{~Hz}, 1 \mathrm{H}) .{ }^{13} \mathbf{C}$ NMR $(125 \mathrm{MHz}$, $\left.\mathrm{CDCl}_{3}\right) \delta 189.74,174.88,151.31,143.01,134.67,132.71,128.77,128.60,128.52,123.19$, 121.49, 117.71, 99.93, 40.75, 36.21, 35.25, 22.81. HRMS: $[\mathrm{M}+\mathrm{H}]^{+}$calcd. For $\mathrm{C}_{17} \mathrm{H}_{15} \mathrm{O}_{4} \mathrm{~S} \mathrm{~m} / \mathrm{z}$ : 315.0691; found: $315.0693 .[\alpha]_{\mathbf{D}}{ }^{20}+90.9\left(c=0.53\right.$ in $\left.\mathrm{CHCl}_{3}\right)$. The enantiomeric excess was determined by HPLC using Chiralpak IB column ( $n$-hexane $/ i$-PrOH $=80 / 20,1 \mathrm{~mL} / \mathrm{min}), \lambda=$ $210 \mathrm{~nm}, t_{\text {major }}=26.69 \mathrm{~min}, t_{\text {minor }}=21.54 \mathrm{~min}, \boldsymbol{e e}=\mathbf{9 9 . 2 \%}$.

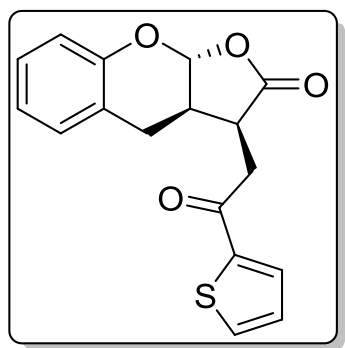

(3S,3aR,9aR)-3-(2-oxo-2-(thiophen-2-yl)ethyl)-3,3a,4,9a-tetrahydro-2 Hfuro[2,3-b]chromen-2-one (9f)

9f ${ }^{n}$ was obtained as a white solid $7 \mathrm{mg}$ in $\mathbf{2 2 \%}$ yield after column chromatography on silica gel with petroleum ether-ethyl acetate $\left(5: 1, \mathrm{R}_{f}=0.1\right)$ as eluant. ${ }^{1} \mathbf{H} \mathbf{~ N M R}\left(500 \mathrm{MHz}, \mathrm{CDCl}_{3}\right)$ $\delta 7.82(\mathrm{dd}, J=7.8,1.7 \mathrm{~Hz}, 1 \mathrm{H}), 7.54-7.47(\mathrm{~m}, 1 \mathrm{H}), 7.20(\mathrm{t}, J=7.7 \mathrm{~Hz}, 1 \mathrm{H}), 7.14(\mathrm{~d}, J=7.3$ $\mathrm{Hz}, 1 \mathrm{H}), 7.03(\mathrm{t}, J=7.1 \mathrm{~Hz}, 2 \mathrm{H}), 6.98(\mathrm{dd}, J=8.2,5.1 \mathrm{~Hz}, 2 \mathrm{H}), 6.29(\mathrm{~d}, J=7.2 \mathrm{~Hz}, 1 \mathrm{H}), 3.91$ (s, 3H), $3.54(\mathrm{dd}, J=18.9,3.3 \mathrm{~Hz}, 1 \mathrm{H}), 3.40(\mathrm{dd}, J=18.9,7.0 \mathrm{~Hz}, 1 \mathrm{H}), 3.15-3.09$ (m, 1H), $2.97(\mathrm{dd}, J=15.3,5.2 \mathrm{~Hz}, 1 \mathrm{H}), 2.78(\mathrm{dd}, J=15.4,1.7 \mathrm{~Hz}, 1 \mathrm{H}), 2.75-2.69(\mathrm{~m}, 1 \mathrm{H}) .{ }^{13} \mathbf{C}$ NMR $\left(125 \mathrm{MHz}, \mathrm{CDCl}_{3}\right) \delta 189.53,177.08,152.41,143.08,134.60,132.61,129.60,128.67$, 
128.49, 123.65, 123.61, 118.45, 100.23, 40.65, 39.17, 37.79, 26.00. HRMS: $[\mathrm{M}+\mathrm{H}]^{+}$calcd.

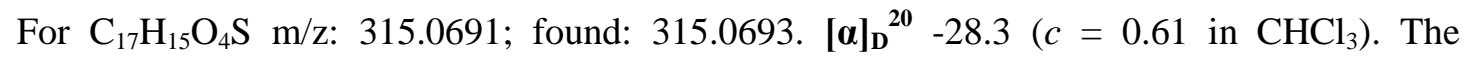
enantiomeric excess was determined by HPLC using Chiralpak IA column ( $n$-hexane/i-PrOH $=80 / 20,1 \mathrm{~mL} / \mathrm{min}), \lambda=210 \mathrm{~nm}, t_{\text {major }}=17.50 \mathrm{~min}, t_{\text {minor }}=21.65 \mathrm{~min}, \boldsymbol{e} \boldsymbol{e}=\mathbf{9 6 . 7 \%}$.

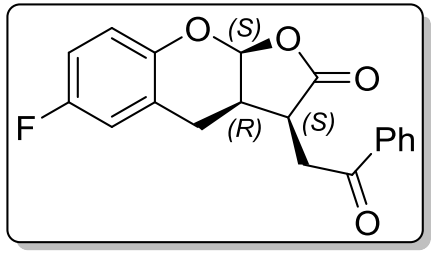

(3S,3aR,9aS)-6-fluoro-3-(2-oxo-2-phenylethyl)-3,3a,4,9a-tetrahydro-2Hfuro[2,3-b]chromen-2-one $(9 \mathrm{~g})$

$11 \mathrm{~g}$ was obtained as a white solid $5.5 \mathrm{mg}$ in $\mathbf{1 7 \%}$ yield after column chromatography on silica gel with petroleum ether-ethyl acetate $\left(6: 1, \mathrm{R}_{f}=0.1\right)$ as eluant. ${ }^{1} \mathbf{H} \mathbf{~ N M R}\left(500 \mathrm{MHz}, \mathrm{CDCl}_{3}\right)$ $\delta 8.00(\mathrm{~d}, J=7.4 \mathrm{~Hz}, 2 \mathrm{H}), 7.65(\mathrm{t}, J=7.3 \mathrm{~Hz}, 1 \mathrm{H}), 7.53(\mathrm{t}, J=7.7 \mathrm{~Hz}, 2 \mathrm{H}), 6.95(\mathrm{dd}, J=8.8$, $4.7 \mathrm{~Hz}, 1 \mathrm{H}), 6.92-6.87(\mathrm{~m}, 1 \mathrm{H}), 6.69(\mathrm{dd}, J=8.1,2.5 \mathrm{~Hz}, 1 \mathrm{H}), 6.25(\mathrm{~d}, J=5.3 \mathrm{~Hz}, 1 \mathrm{H})$, $3.68-3.57(\mathrm{~m}, 2 \mathrm{H}), 3.36-3.28(\mathrm{~m}, 1 \mathrm{H}), 3.23(\mathrm{dd}, J=18.9,10.9 \mathrm{~Hz}, 1 \mathrm{H}), 2.66(\mathrm{dd}, J=15.8$, $6.8 \mathrm{~Hz}, 1 \mathrm{H}), 2.40(\mathrm{dd}, J=15.7,8.1 \mathrm{~Hz}, 1 \mathrm{H}) .{ }^{13} \mathbf{C} \mathbf{N M R}\left(125 \mathrm{MHz}, \mathrm{CDCl}_{3}\right) \delta$ 196.87, 174.90, $159.30,157.38,147.18,147.16,135.87,134.14,129.09,128.26,123.41,123.34,119.03$, 118.96, 115.48, 115.29, 114.85, 114.66, 100.03, 40.57, 36.07, 34.88, 23.06. HRMS: $[\mathrm{M}+\mathrm{H}]^{+}$ calcd. For $\mathrm{C}_{19} \mathrm{H}_{16} \mathrm{FO}_{4} \mathrm{~m} / \mathrm{z}$ : 327.1033; found: 327.1035 . [ $\left.\alpha\right]_{\mathbf{D}}{ }^{20}+74.1\left(c=0.47\right.$ in $\left.\mathrm{CHCl}_{3}\right)$. The enantiomeric excess was determined by HPLC using Chiralpak IA column ( $n$-hexane/i-PrOH $=80 / 20,1 \mathrm{~mL} / \mathrm{min}), \lambda=210 \mathrm{~nm}, t_{\text {major }}=16.00 \mathrm{~min}, t_{\text {minor }}=19.85 \mathrm{~min}, \boldsymbol{e} \boldsymbol{e}=\mathbf{9 9 . 2 \%}$.

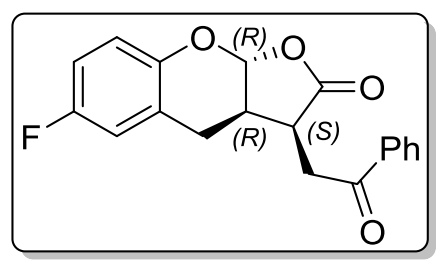

(3S,3aR,9aR)-6-fluoro-3-(2-oxo-2-phenylethyl)-3,3a,4,9a-tetrahydro-2 $\mathrm{H}$ furo[2,3-b]chromen-2-one $\left(9 g^{\prime}\right)$

9g` was obtained as a white solid $6 \mathrm{mg}$ in $\mathbf{1 8 \%}$ yield after column chromatography on silica gel with petroleum ether-ethyl acetate $\left(5: 1, \mathbf{R}_{f}=0.1\right)$ as eluant. ${ }^{1} \mathbf{H} \mathbf{~ N M R}\left(500 \mathrm{MHz}, \mathrm{cdcl}_{3}\right) \delta$ $8.00(\mathrm{~d}, J=7.4 \mathrm{~Hz}, 2 \mathrm{H}), 7.65(\mathrm{t}, J=7.3 \mathrm{~Hz}, 1 \mathrm{H}), 7.53(\mathrm{t}, J=7.7 \mathrm{~Hz}, 2 \mathrm{H}), 6.95(\mathrm{dd}, J=8.8$, $4.7 \mathrm{~Hz}, 1 \mathrm{H}), 6.92-6.87(\mathrm{~m}, 1 \mathrm{H}), 6.69(\mathrm{dd}, J=8.1,2.5 \mathrm{~Hz}, 1 \mathrm{H}), 6.25(\mathrm{~d}, J=5.3 \mathrm{~Hz}, 1 \mathrm{H})$, $3.68-3.57(\mathrm{~m}, 2 \mathrm{H}), 3.36-3.28(\mathrm{~m}, 1 \mathrm{H}), 3.23(\mathrm{dd}, J=18.9,10.9 \mathrm{~Hz}, 1 \mathrm{H}), 2.66(\mathrm{dd}, J=15.8$, 
$6.8 \mathrm{~Hz}, 1 \mathrm{H}), 2.40(\mathrm{dd}, J=15.7,8.1 \mathrm{~Hz}, 1 \mathrm{H}) .{ }^{13} \mathbf{C} \mathbf{N M R}\left(125 \mathrm{MHz}, \mathrm{CDCl}_{3}\right) \delta 196.63,177.06$, $159.70,157.78,148.19,148.17,135.97,134.02,128.99,128.21,125.89,125.82,119.75$, $119.68,116.25,116.07,115.21,115.03,100.42,40.47,38.86,37.90,26.43$. HRMS: $[\mathrm{M}+\mathrm{H}]^{+}$

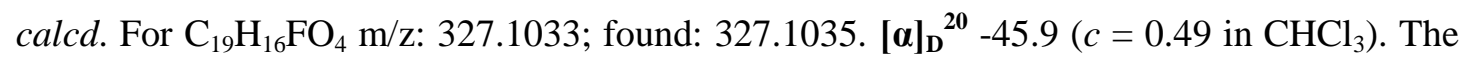
enantiomeric excess was determined by HPLC using Chiralpak IC column ( $n$-hexane/i-PrOH $=70 / 30,1 \mathrm{~mL} / \mathrm{min}), \lambda=210 \mathrm{~nm}, t_{\text {major }}=26.05 \mathrm{~min}, t_{\text {minor }}=31.06 \mathrm{~min}, \boldsymbol{e} \boldsymbol{e}=\mathbf{9 6 . 3 \%}$. 


\section{Single Crystal X-Ray Diffraction Data for Compound 11}

[CCDC 143837711 contains the supplementary crystallographic data for this paper. These data can be obtained free of charge from The Cambridge Crystallographic Data Centre via www.ccdc.cam.ac.uk/data_request/cif.].
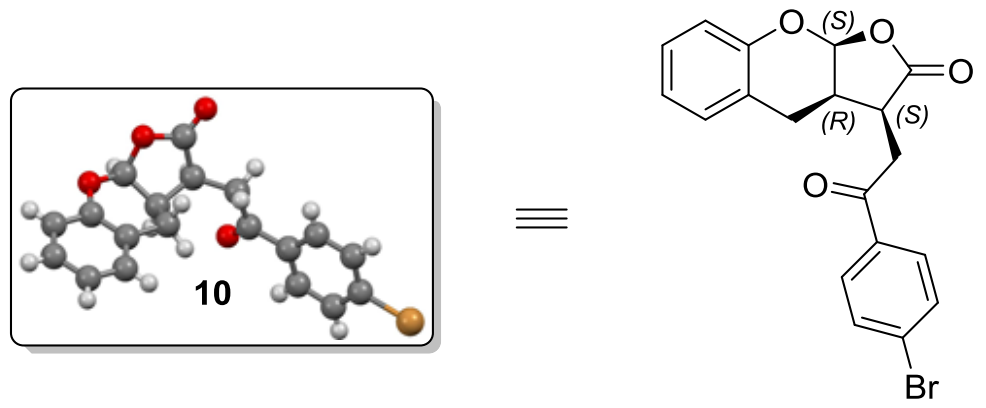

$\begin{array}{llll}\text { Bond precision: } & \mathrm{C}-\mathrm{C}=0.0137 \mathrm{~A} & \\ \text { Cell: } & \mathrm{a}=10.8044(14) & \mathrm{b}=6.7767(8) & \mathrm{C}=12.3031(14) \\ & \text { alpha=90 } & \text { beta=113.354(15) } & \text { gamma=90 }\end{array}$

Temperature: $293 \mathrm{~K}$

Calculated

Volume

Space group

Hall group

Moiety formula

Sum formula

$\mathrm{Mr}$

Dx,g cm-3

Z

$\mathrm{Mu}(\mathrm{mm}-1)$

F000

F000'

h,k, Imax

Nref

Tmin,Tmax

Tmin'
827.0(2)

P 21

$\mathrm{P} 2 \mathrm{yb}$

C19 H15 Br O4

C19 H15 Br O4

387.21

1.555

2

3.563

392.0

391.69

$12,8,14$

2967[ 1621]

$0.752,0.779$

0.752

Wavelength $=1.54184$

Correction method $=\#$ Reported $\mathrm{T}$ Limits: $\mathrm{Tmin}=0.764 \mathrm{Tmax}=0.789$ AbsCorr $=$ MULTI-SCAN

Data completeness $=1.30 / 0.71$

$\mathrm{R}($ reflections $)=0.0577(1576)$

Theta $(\max )=67.250$

$\mathrm{S}=1.098$

Npar $=218$

Reported

827.01(18)

$\mathrm{P} 21$

?

?

C19 H15 Br O4

387.22

1.555

2

3.563

392.0

$12,8,14$

2101

$0.764,0.789$ 


\section{NMR spectra and HPLC Traces}
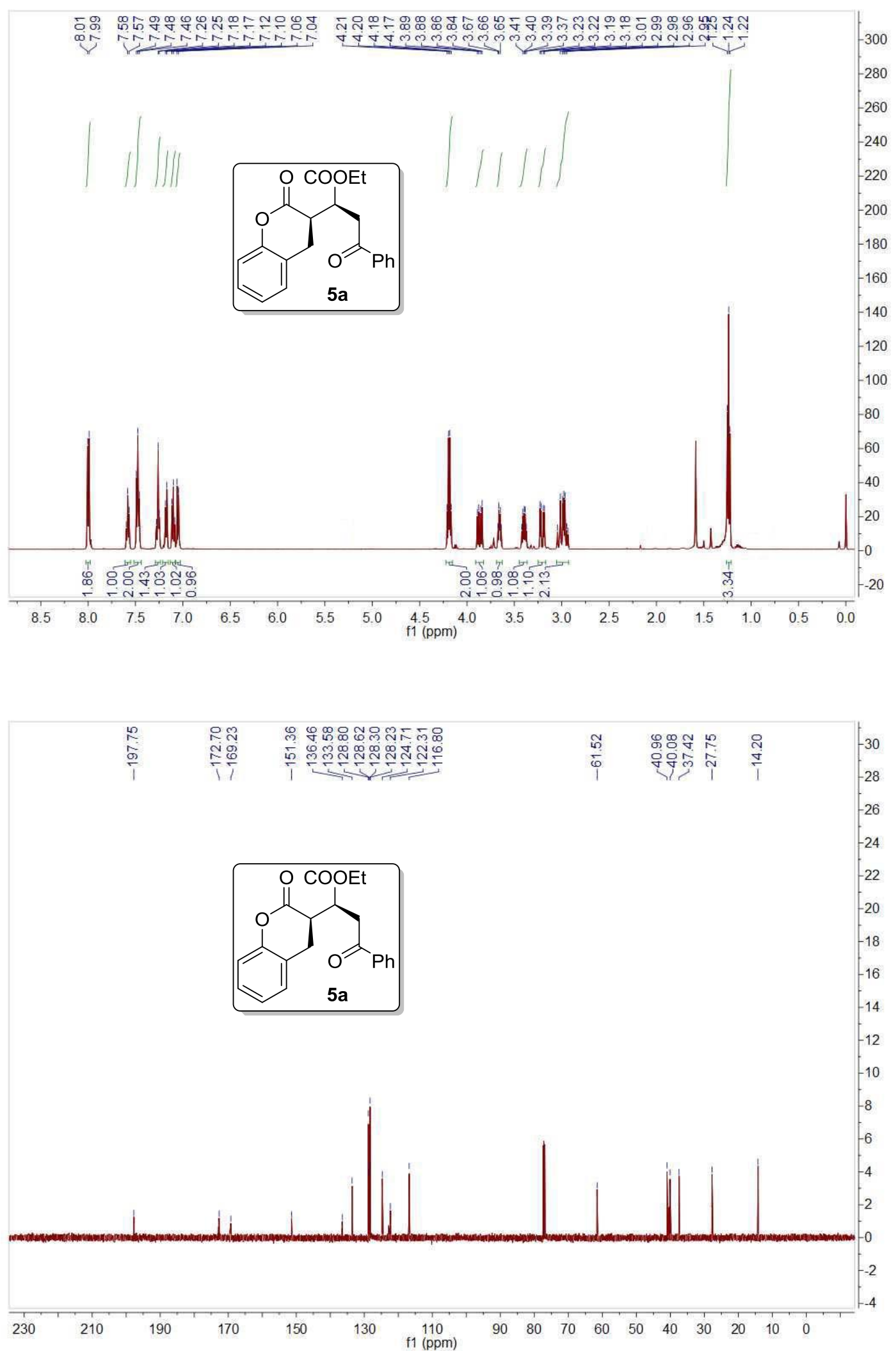
Chrom Type: Fixed WL Chromatogram, $210 \mathrm{~nm}$

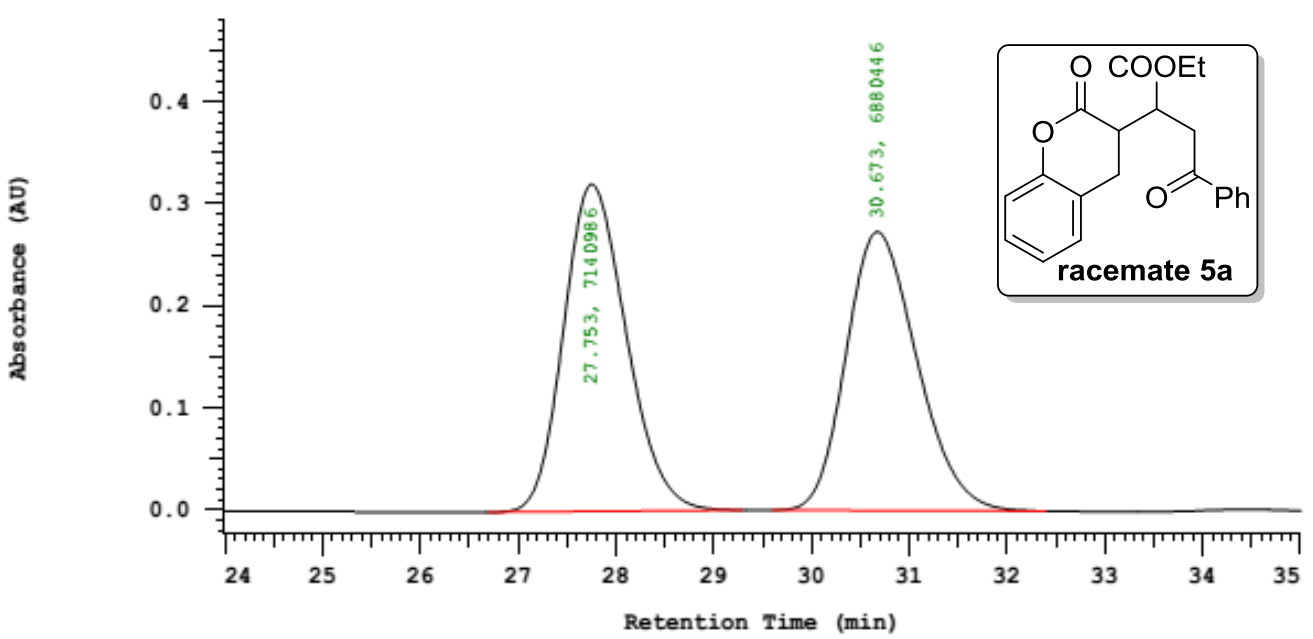

Chrom Type: Fixed WL Chromatogram, $210 \mathrm{~nm}$

Peak Quantitation: AREA

Calculation Method: AREA

\begin{tabular}{ccccc} 
No. & RT & Area & Area 8 & BC \\
\hline 1 & 27.753 & 7140986 & 50.929 & BB \\
2 & 30.673 & 6880446 & 49.071 & BB \\
\hline & 14021432 & 100.000 & \\
\hline
\end{tabular}

Chrom Type: Fixed WL Chromatogram, $210 \mathrm{~nm}$

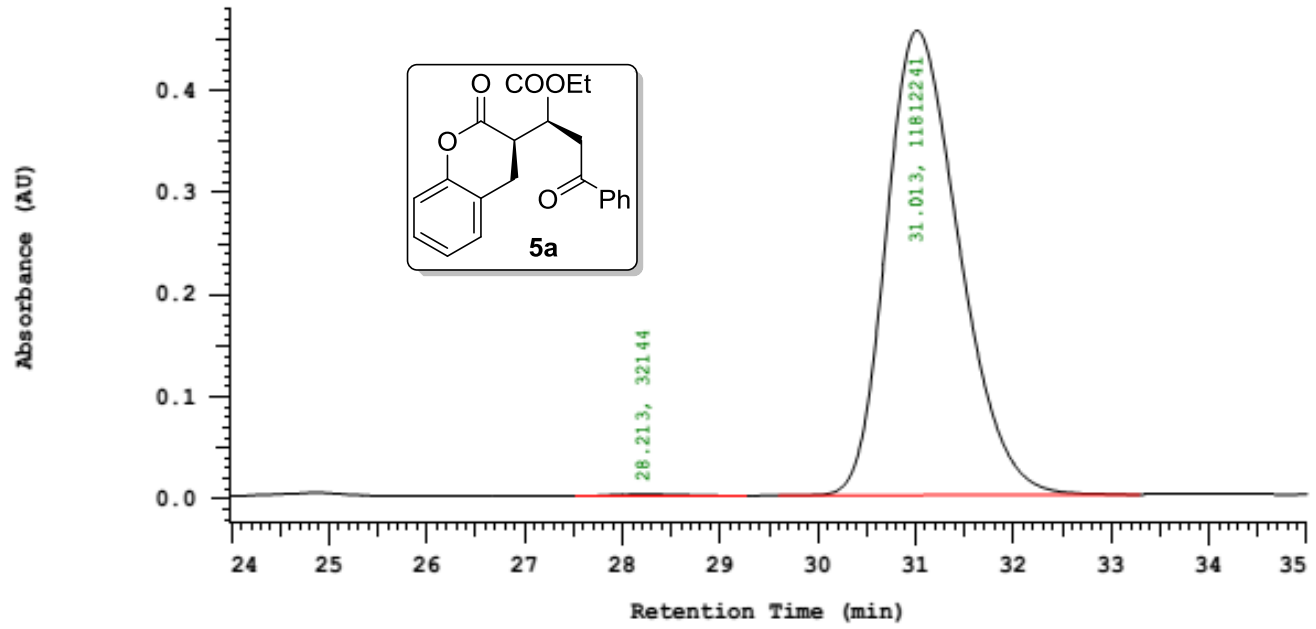

Chrom Type: Fixed WL Chromatogram, $210 \mathrm{~nm}$

Peak Quantitation: AREA

Calculation Method: AREA

\begin{tabular}{rrrrr} 
No. & RT & \multicolumn{1}{c}{ Area } & Area \& & BC \\
\hline 1 & 28.213 & 32144 & 0.271 & BB \\
2 & 31.013 & 11812241 & 99.729 & BB \\
\hline & & 11844385 & 100.000 & \\
\hline
\end{tabular}



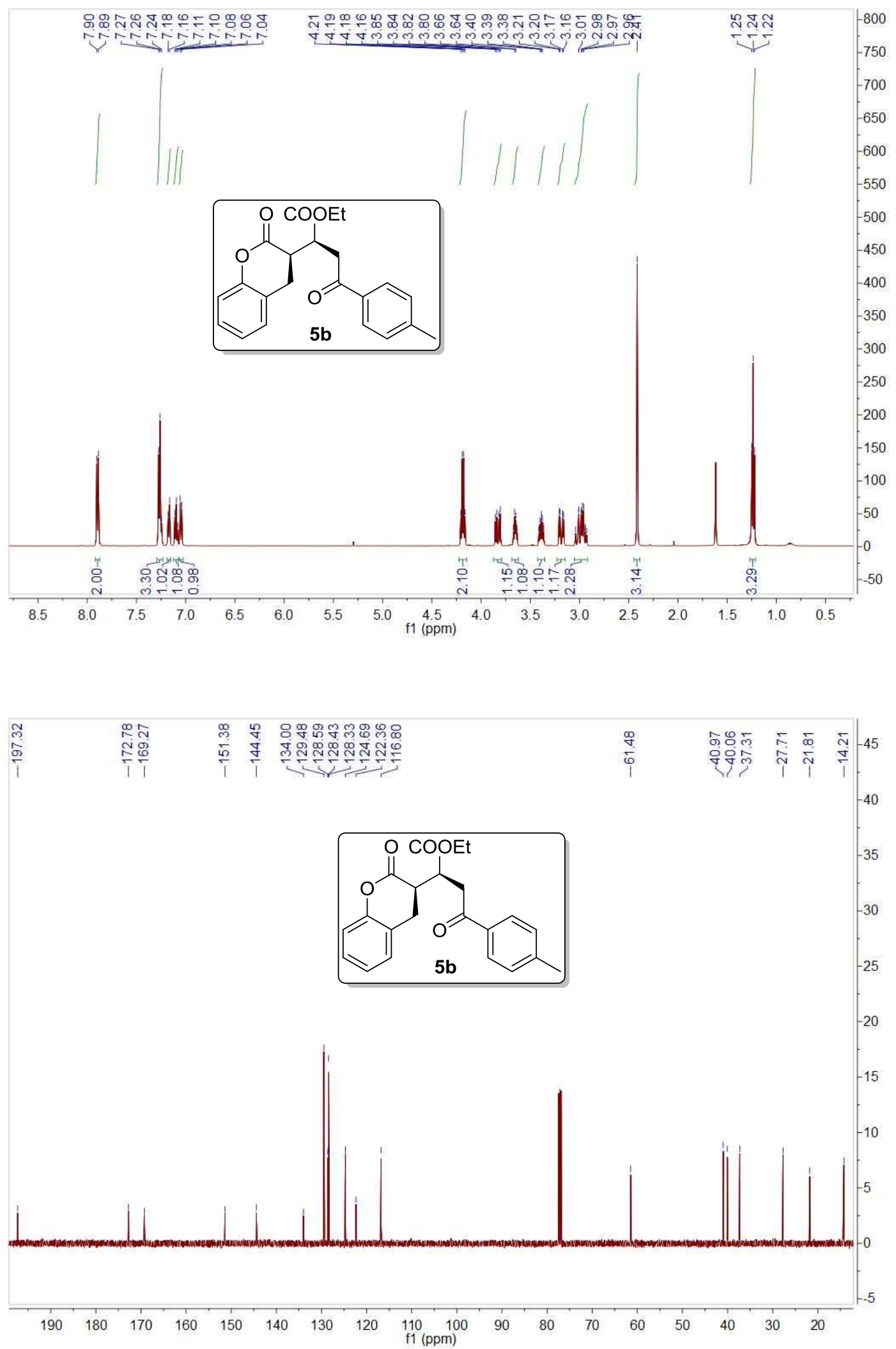
Chrom Type: Fixed WL Chromatogram, $210 \mathrm{~nm}$

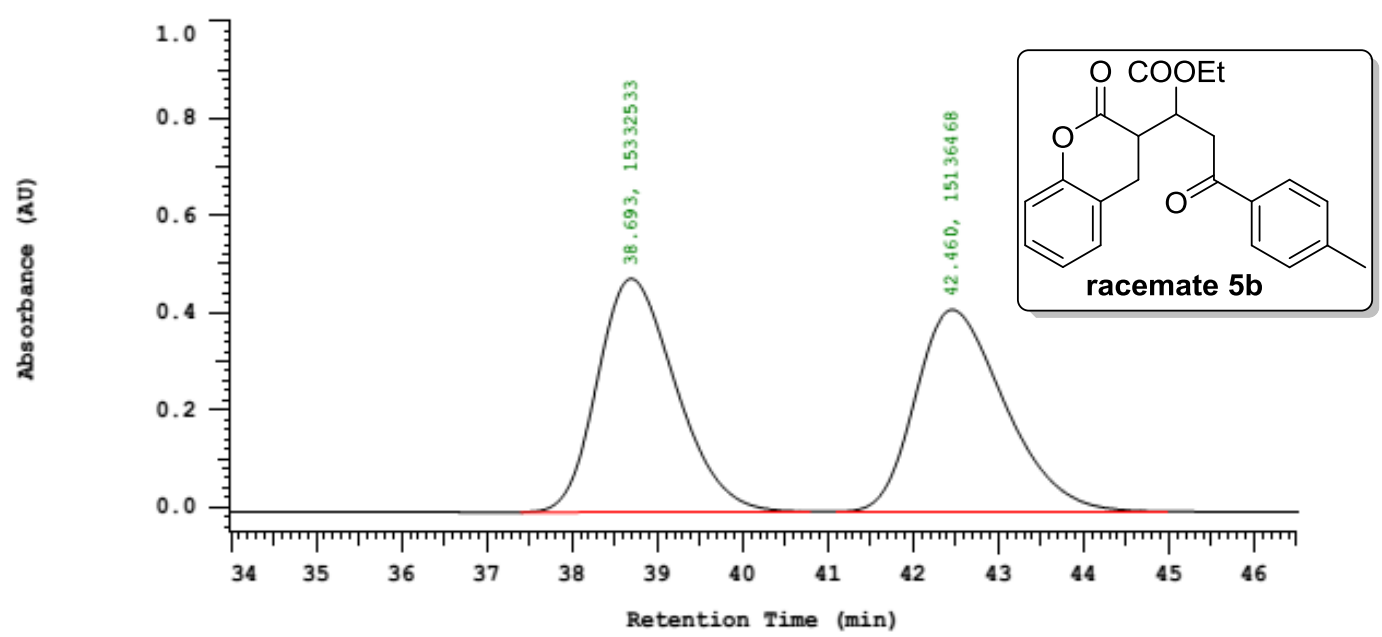

Chrom Type: Fixed WL Chromatogram, $210 \mathrm{~nm}$

Peak Quantitation: AREA

Calculation Method: AREA

\begin{tabular}{ccccc} 
No. & RT & Area & Area 8 & BC \\
\hline 1 & 38.693 & 15332533 & 50.322 & BB \\
2 & 42.460 & 15136468 & 49.678 & BB \\
\hline & 30469001 & 100.000 & \\
\hline
\end{tabular}

Chrom Type: Fixed WL Chromatogram, $210 \mathrm{~nm}$

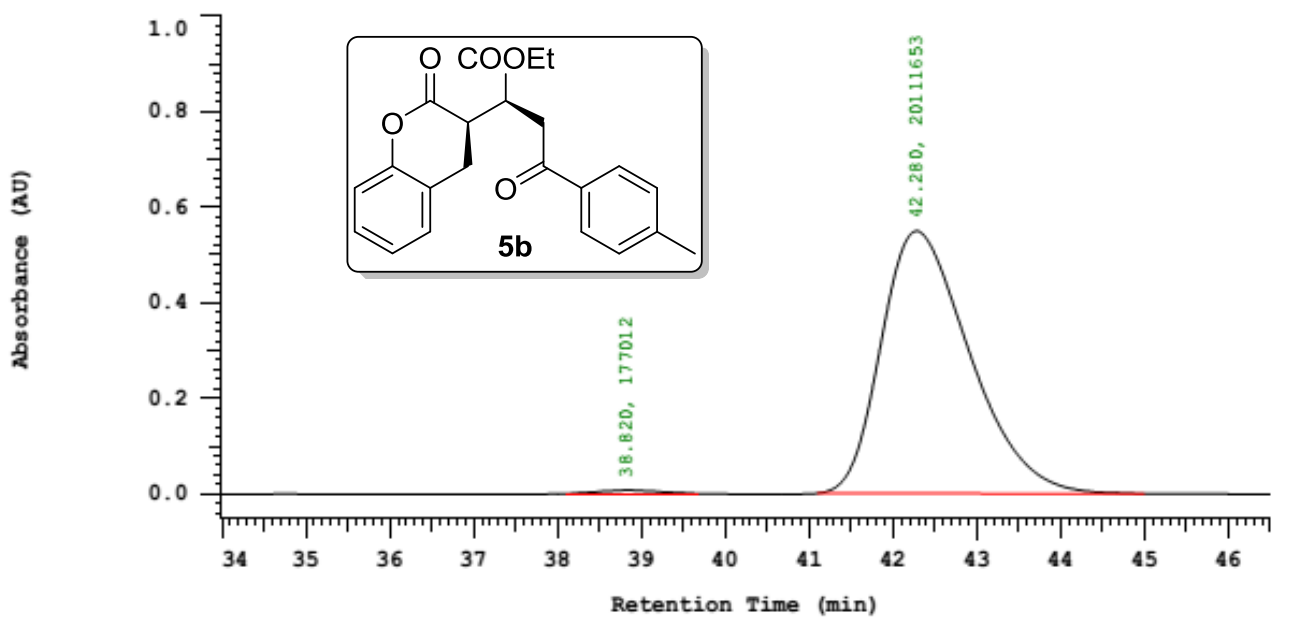

Chrom Type: Fixed WL Chromatogram, $210 \mathrm{~nm}$

Peak Quantitation: AREA

Calculation Method: AREA

\begin{tabular}{ccccc} 
No. & RT & Area & Area o & BC \\
\hline 1 & 38.820 & 177012 & 0.872 & BB \\
2 & 42.280 & 20111653 & 99.128 & BB \\
\hline & 20288665 & 100.000 & \\
\hline
\end{tabular}



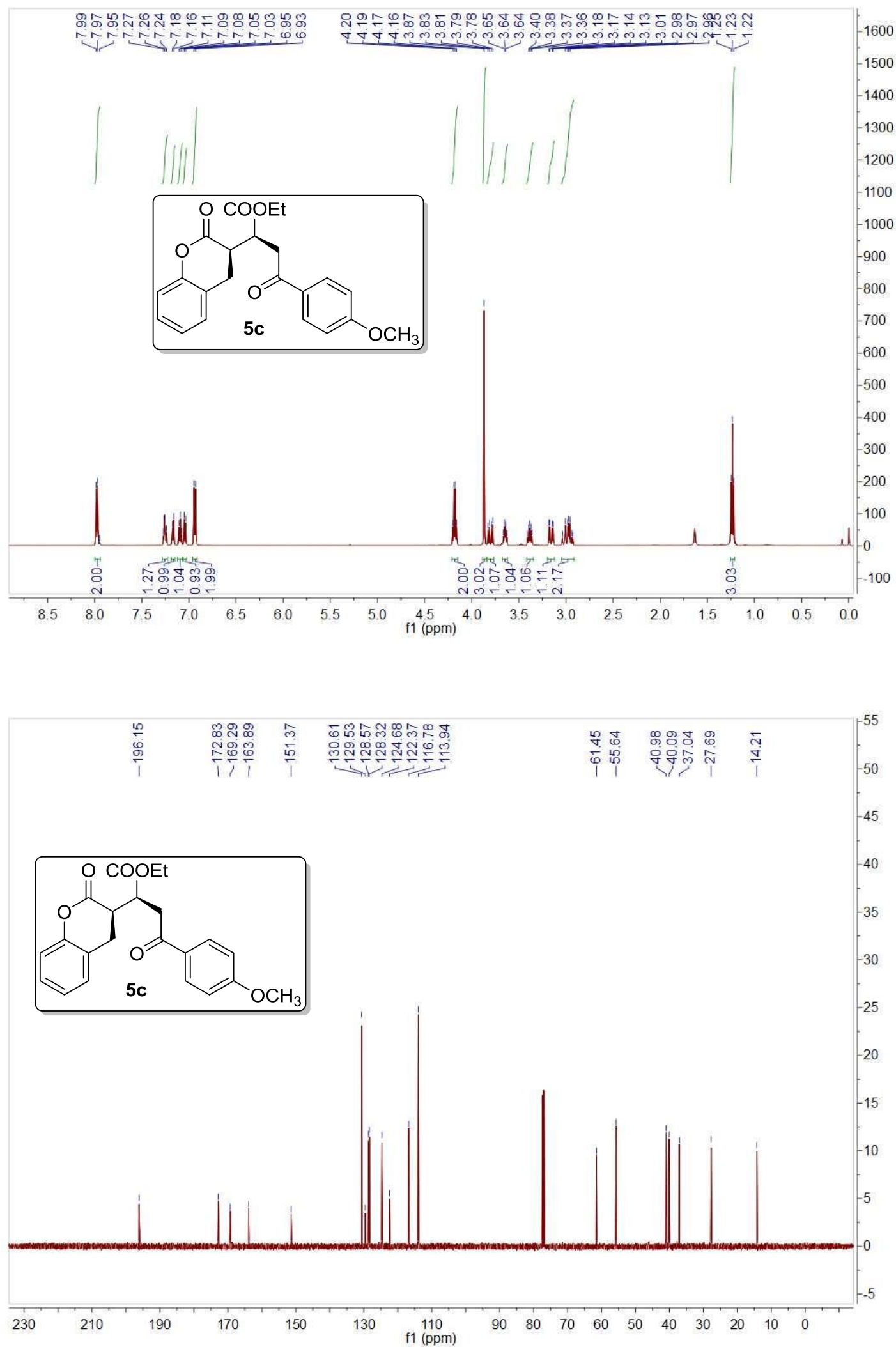
Chrom Type: Fixed WL Chromatogram, $210 \mathrm{~nm}$

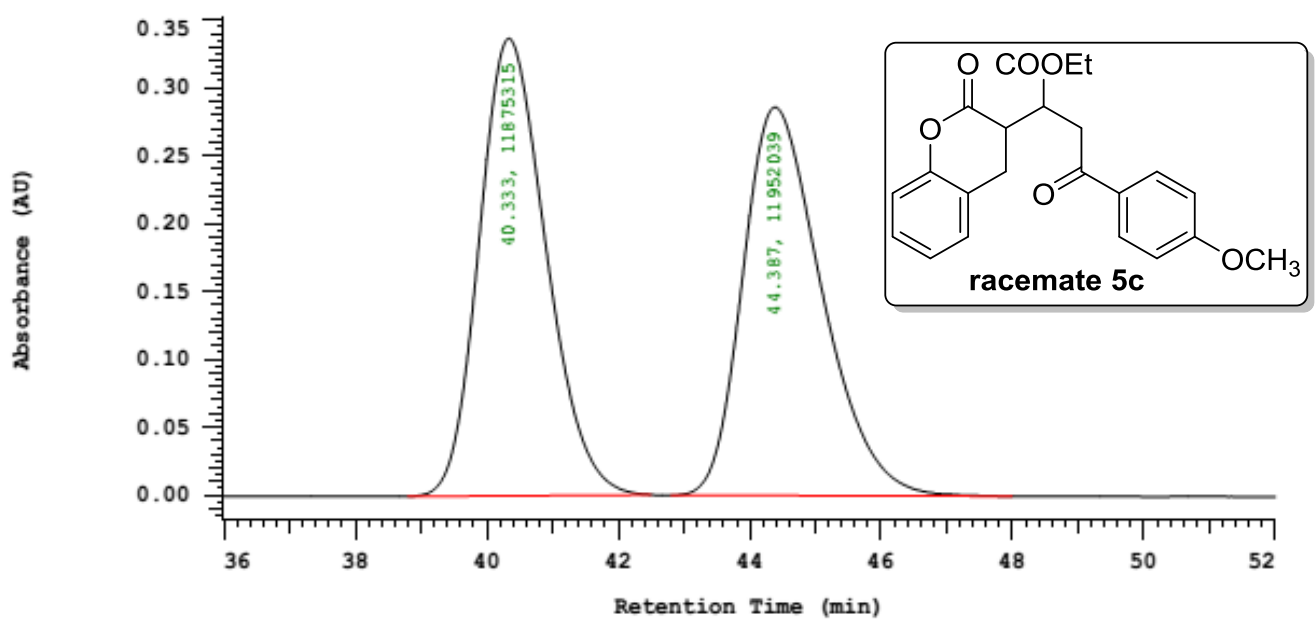

Chrom Type: Fixed WL Chromatogram, $210 \mathrm{~nm}$

Peak Quantitation: AREA

Calculation Method: AREA\&

\begin{tabular}{ccccc} 
No. & RT & Area & Area 8 & BC \\
\hline 1 & 40.333 & 11875315 & 49.839 & BB \\
2 & 44.387 & 11952039 & 50.161 & BB \\
\hline & & 23827354 & 100.000 & \\
\hline
\end{tabular}

Chrom Type: Fixed WL Chromatogram, $210 \mathrm{~nm}$

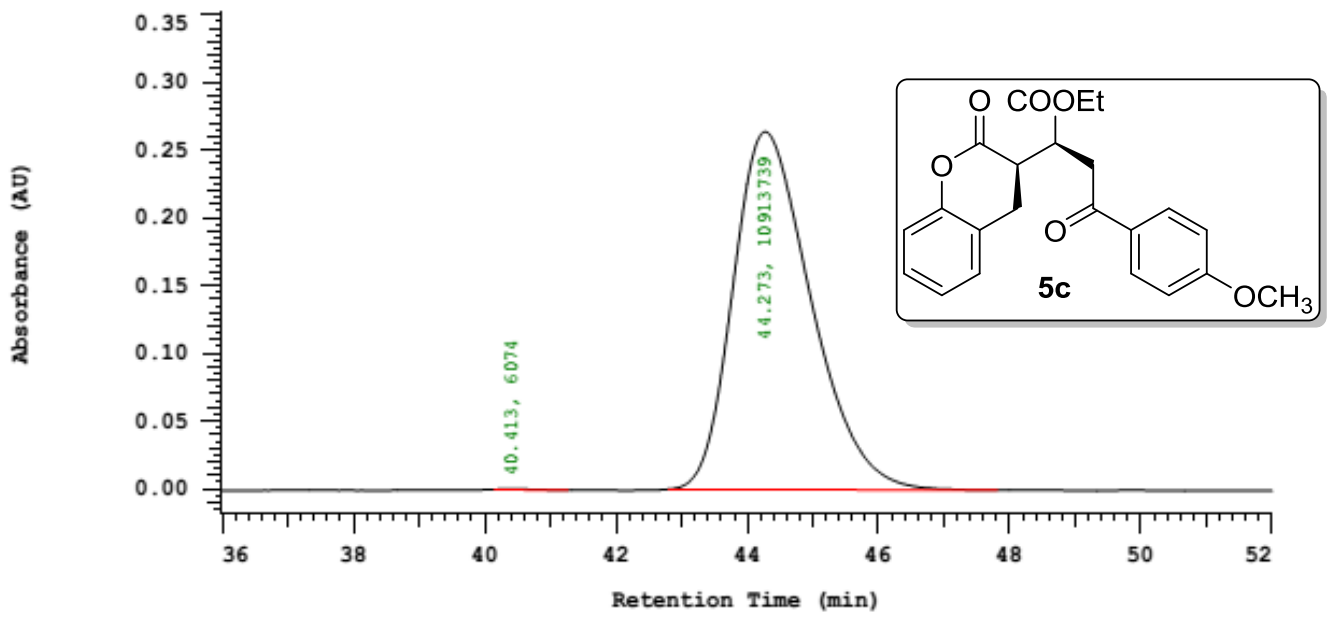

Chrom Type: Fixed WL Chromatogram, $210 \mathrm{~nm}$

Peak Quantitation: AREA

Calculation Method: AREA

\begin{tabular}{rrrrr} 
No. & RT & \multicolumn{1}{c}{ Area } & Area \& & BC \\
\hline 1 & 40.413 & 6074 & 0.056 & BB \\
2 & 44.273 & 10913739 & 99.944 & BB \\
\hline & & 10919813 & 100.000 & \\
\hline
\end{tabular}



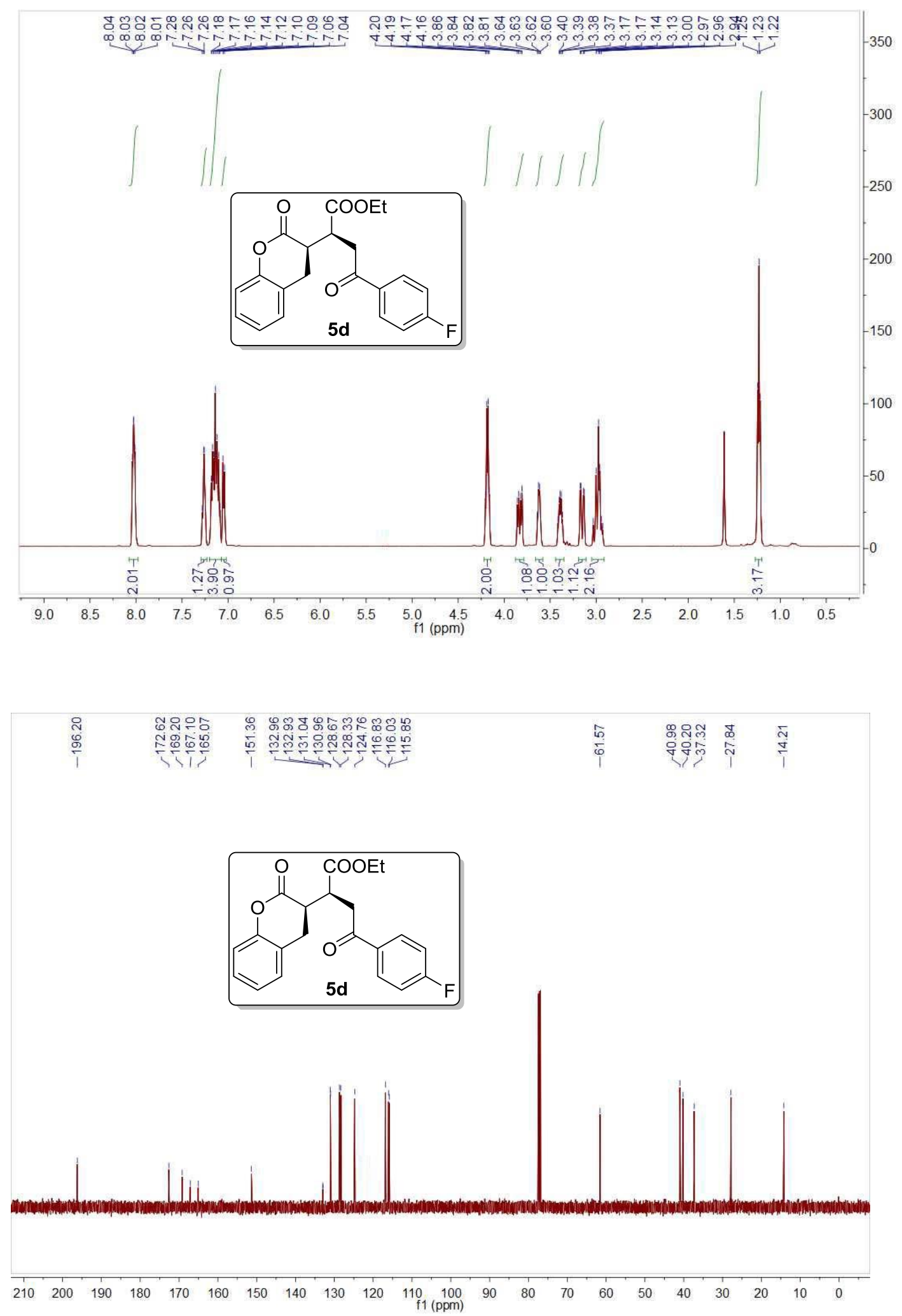
Chrom Type: Fixed WL Chromatogram, $210 \mathrm{~nm}$

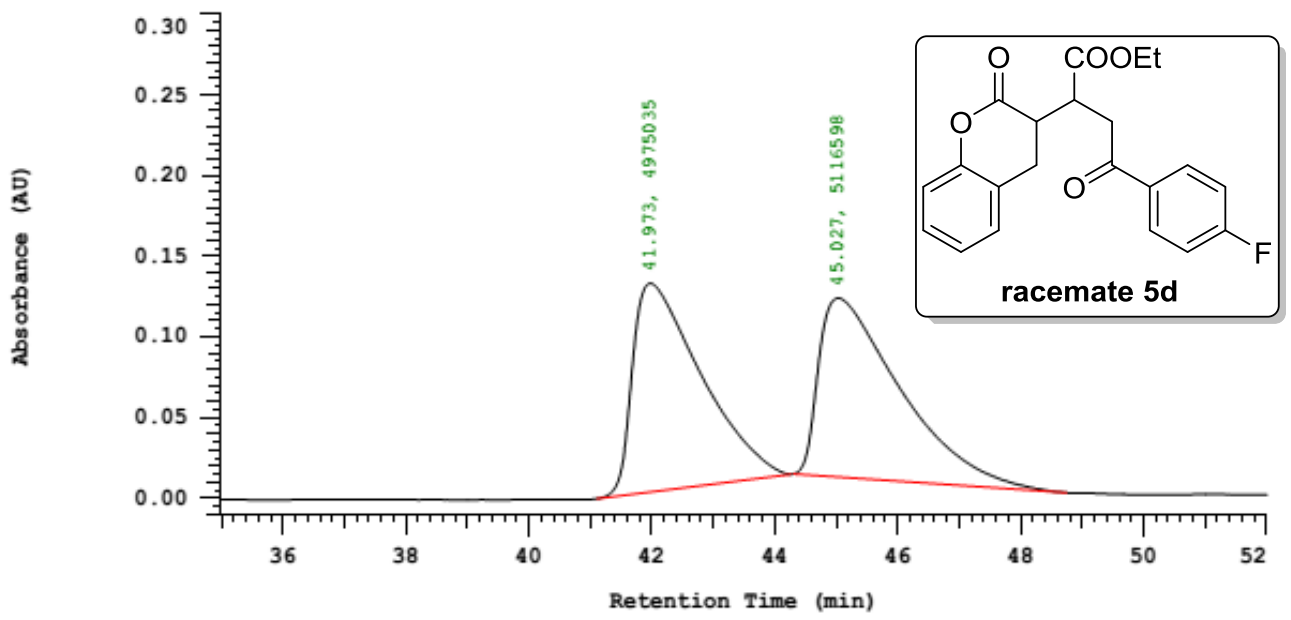

Chrom Type: Fixed WL Chromatogram, $210 \mathrm{~nm}$

Peak Quantitation: AREA

Calculation Method: AREA\&

\begin{tabular}{ccccc} 
No. & RT & Area & Area 8 & BC \\
\hline 1 & 41.973 & 4975035 & 49.299 & BB \\
2 & 45.027 & 5116598 & 50.701 & BB \\
\hline & & 10091633 & 100.000 & \\
\hline
\end{tabular}

Chrom Type: Fixed WL Chromatogram, $210 \mathrm{~nm}$

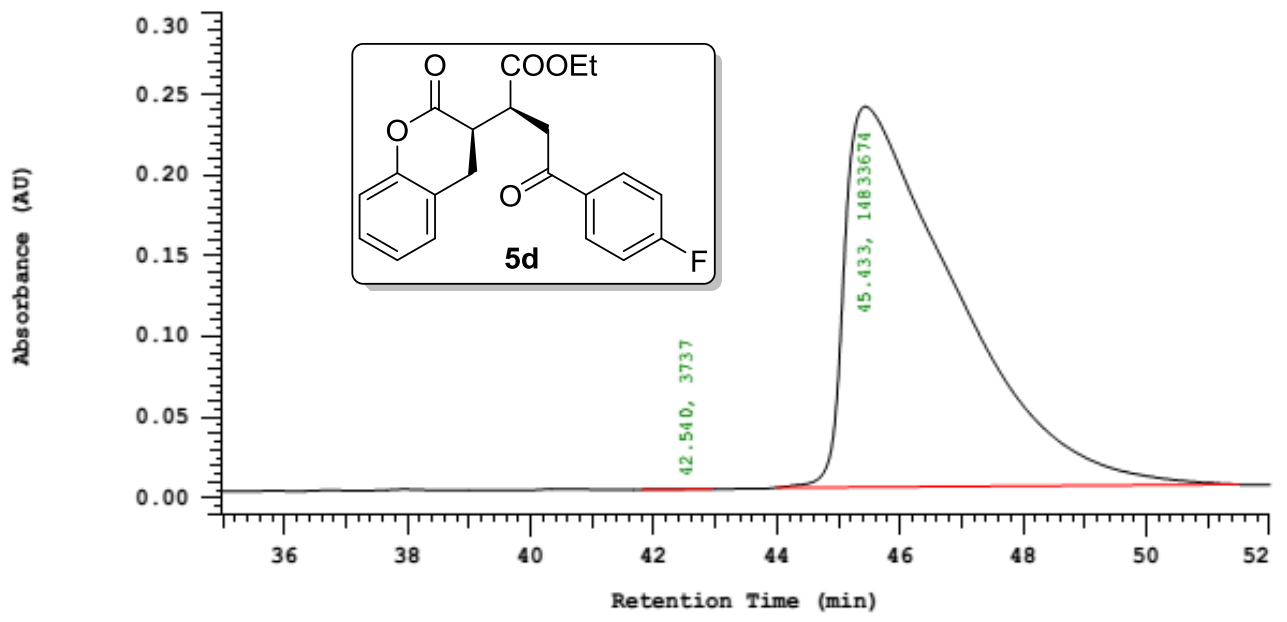

Chrom Type: Fixed WL Chromatogram, $210 \mathrm{~nm}$

Peak Quantitation: AREA

Calculation Method: AREA\&

\begin{tabular}{|c|c|c|c|c|}
\hline No. & $\mathrm{RT}$ & Area & Area \& & $\mathrm{BC}$ \\
\hline 1 & 42.540 & 3737 & 0.025 & BB \\
\hline \multirow[t]{2}{*}{2} & 45.433 & 14833674 & 99.975 & BB \\
\hline & & 14837411 & 100.000 & \\
\hline
\end{tabular}



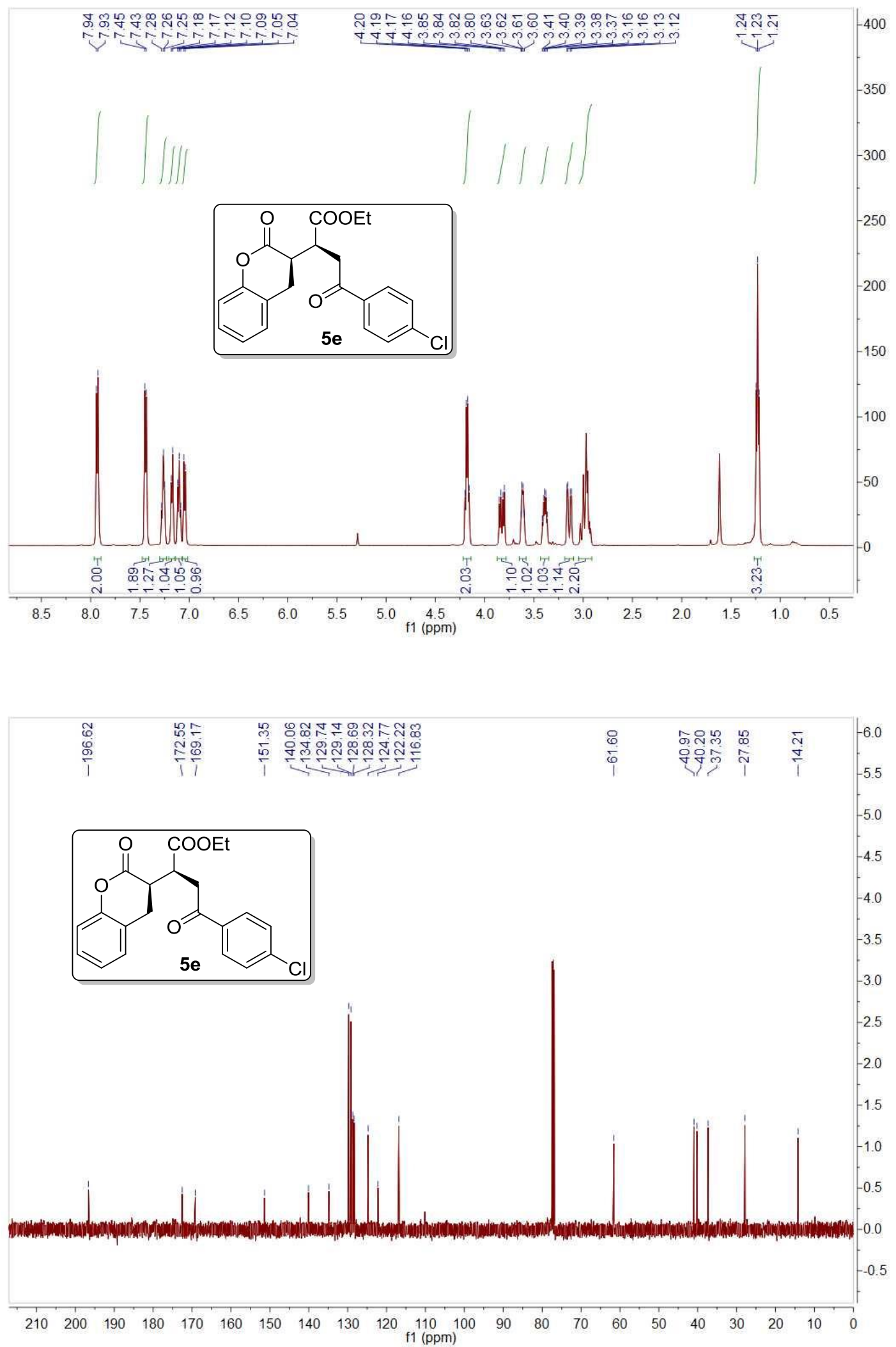
Chrom Type: Fixed WL Chromatogram, $210 \mathrm{~nm}$

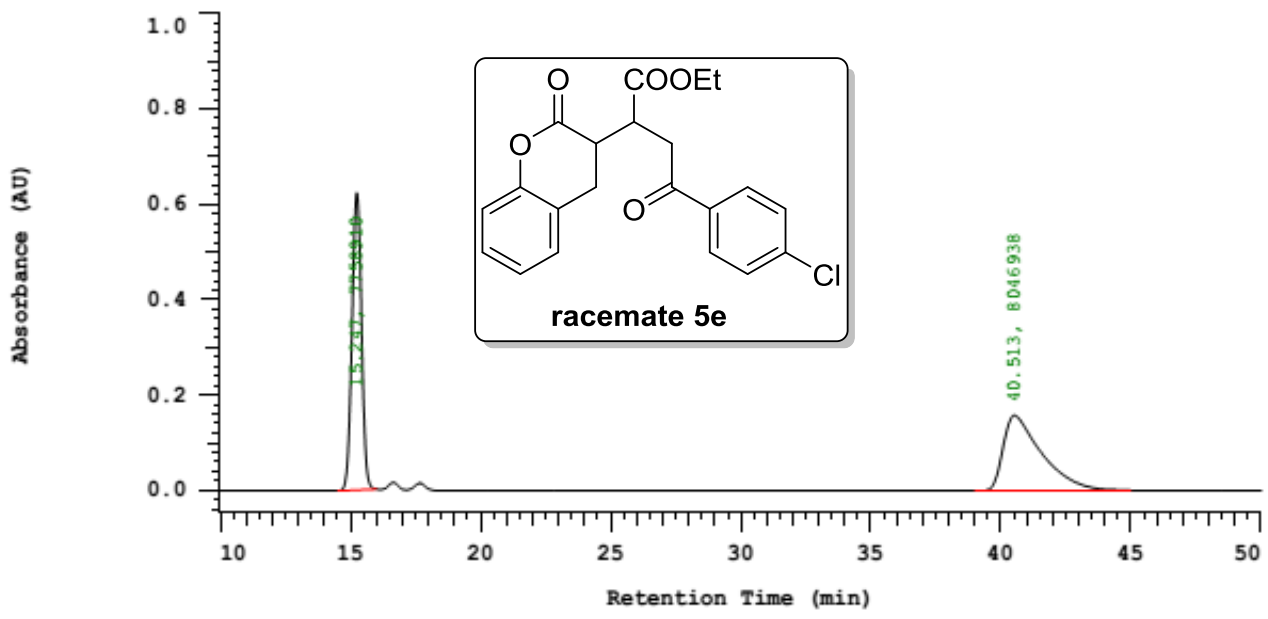

Peak Quantitation: AREA

Chrom Type: Fixed WL Chromatogram, $210 \mathrm{~nm}$ Calculation Method: AREA

\begin{tabular}{ccccc} 
No. & RT & Area & Area 8 & BC \\
\hline 1 & 15.247 & 7758910 & 49.089 & BB \\
2 & 40.513 & 8046938 & 50.911 & BB \\
\hline & 15805848 & 100.000 & \\
\hline
\end{tabular}

Chrom Type: Fixed WL Chromatogram, $210 \mathrm{~nm}$

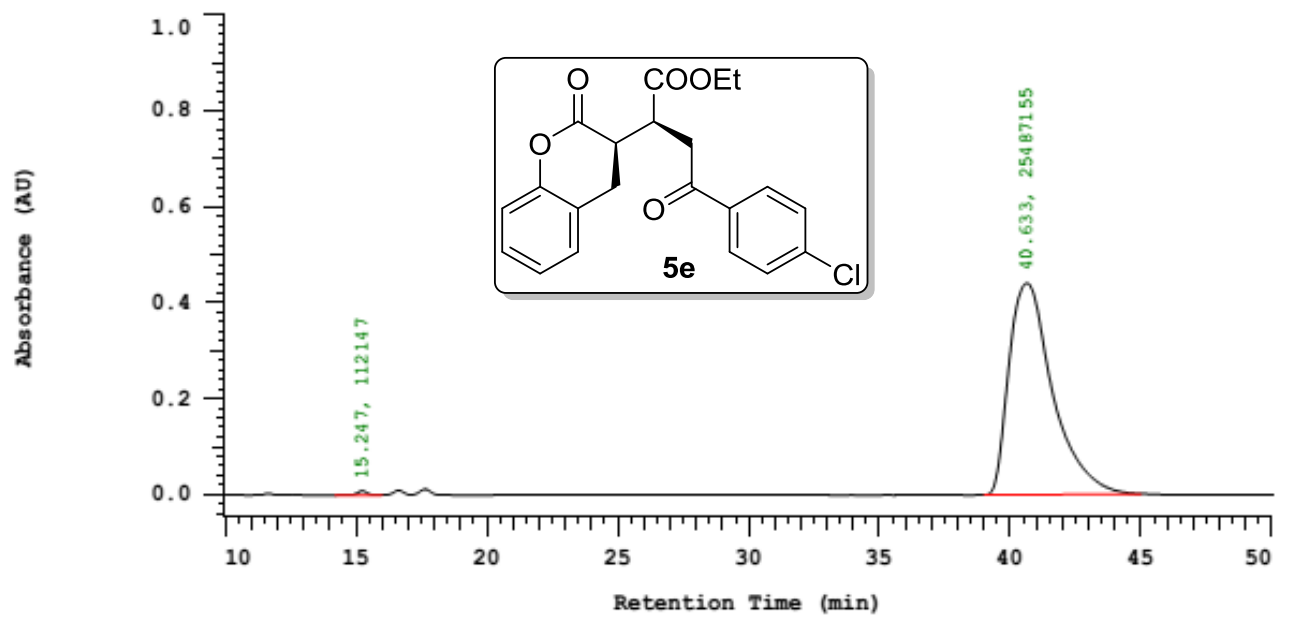

Chrom Type: Fixed WL Chromatogram, $210 \mathrm{~nm}$

Peak Quantitation: AREA

Calculation Method: AREA\&

\begin{tabular}{ccrrr} 
No. & RT & \multicolumn{1}{c}{ Area } & Area \& & BC \\
\hline 1 & 15.247 & 112147 & 0.438 & BB \\
2 & 40.633 & 25487155 & 99.562 & BB \\
\hline & & 25599302 & 100.000 & \\
\hline
\end{tabular}



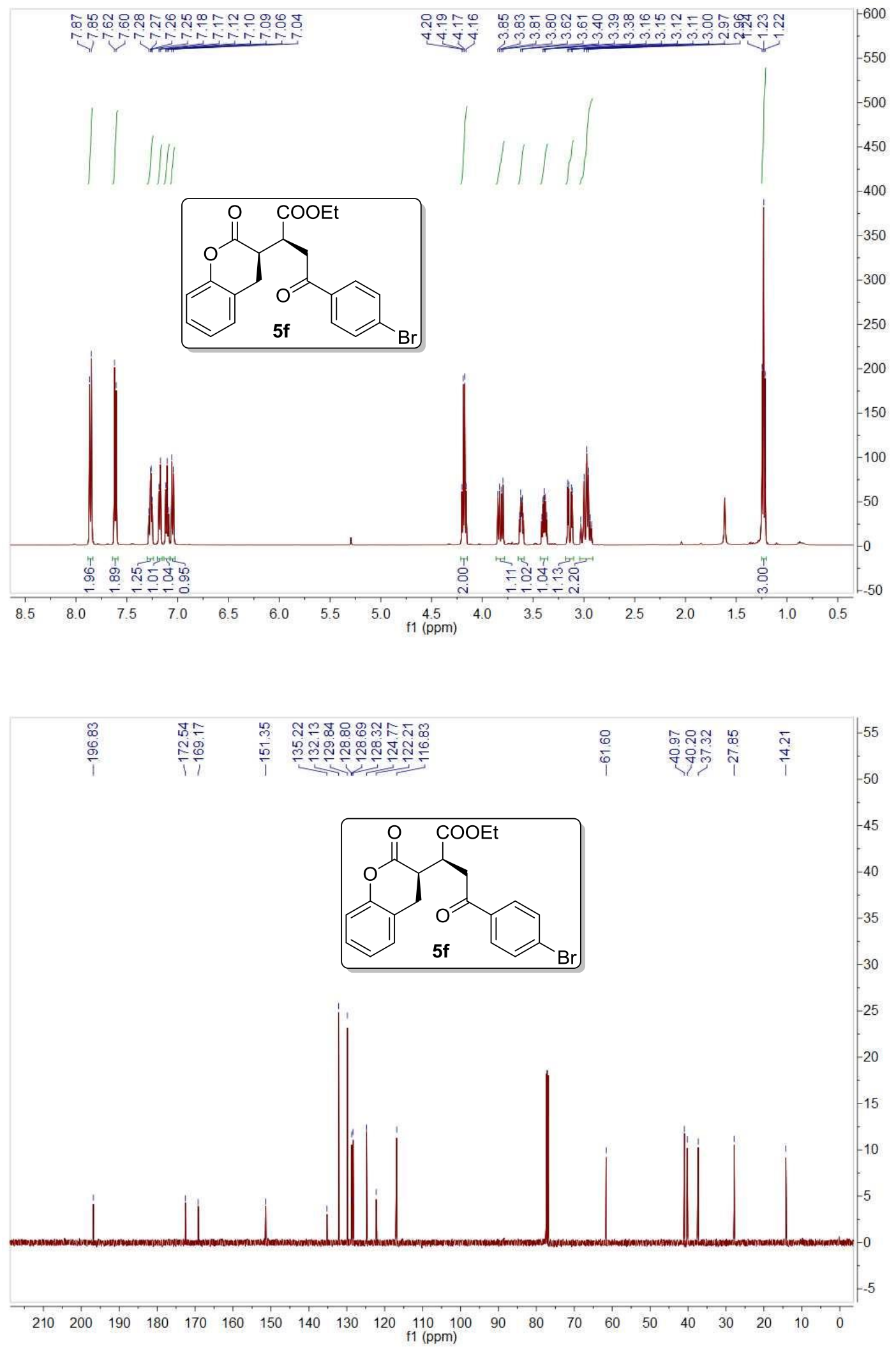
Chrom Type: Fixed WL Chromatogram, 210 nm

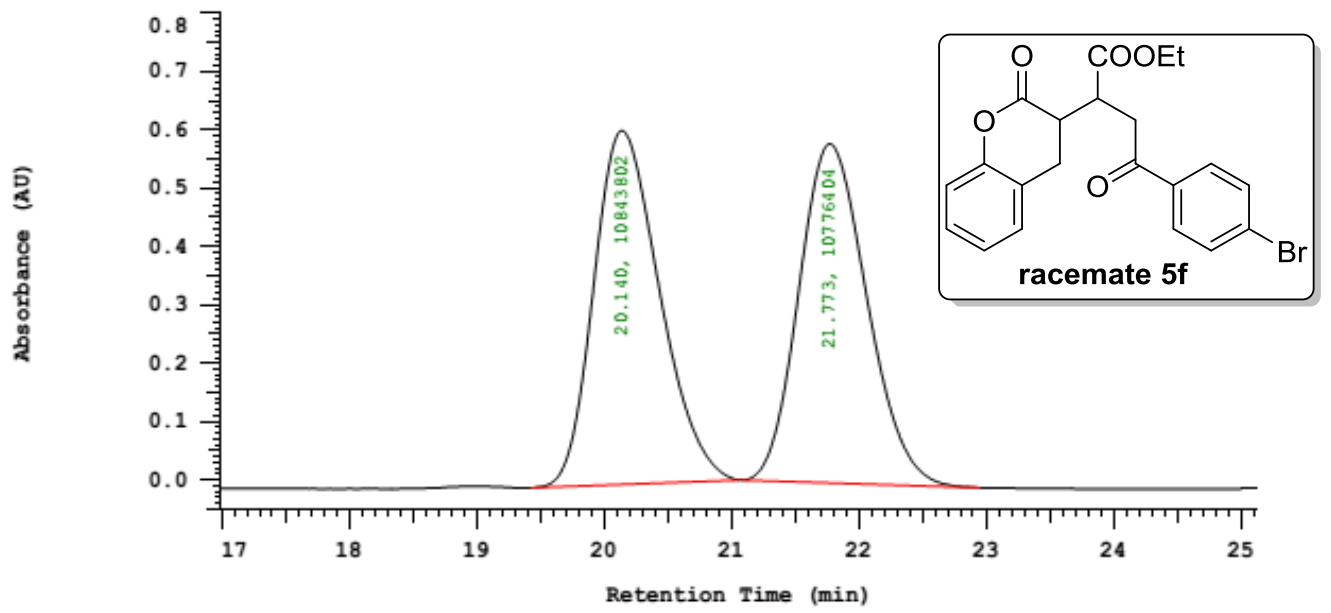

Chrom Type: Fixed WL Chromatogram, $210 \mathrm{~nm}$

Peak Quantitation: AREA

Calculation Method: AREA용

\begin{tabular}{ccccc} 
No. & RT & Area & Area 8 & BC \\
\hline 1 & 20.140 & 10843802 & 50.156 & BB \\
2 & 21.773 & 10776404 & 49.844 & BB \\
\hline & 21620206 & 100.000 & \\
\hline
\end{tabular}

Chrom Type: Fixed WL Chromatogram, $210 \mathrm{~nm}$

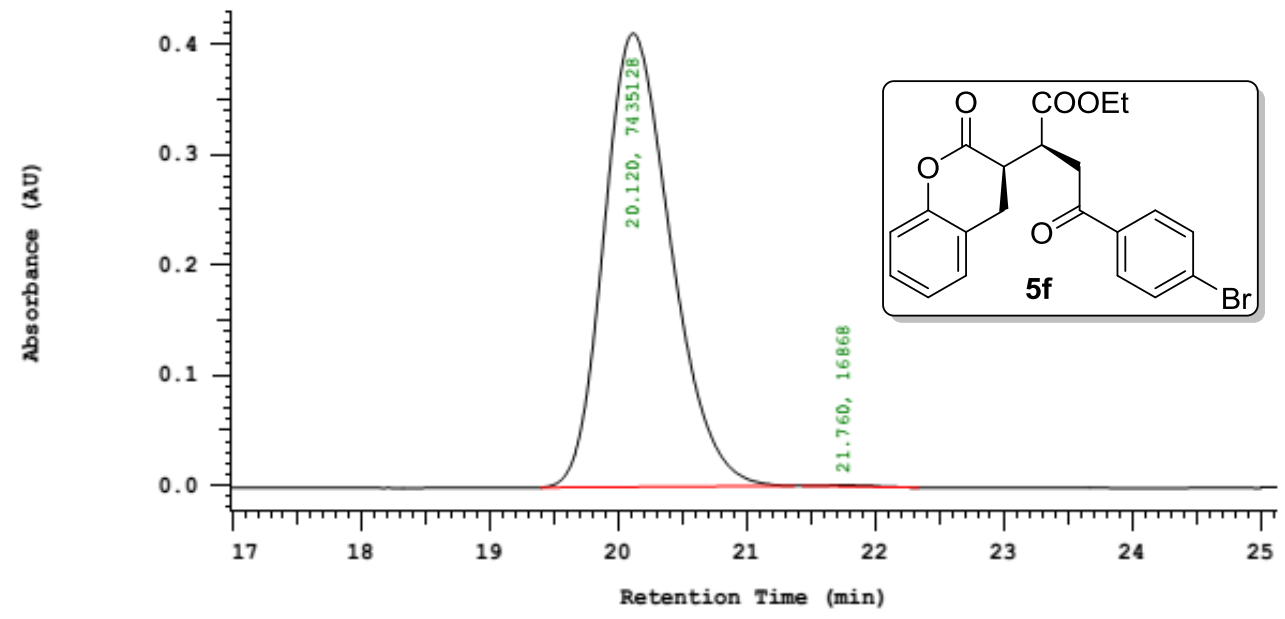

Chrom Type: Fixed WL Chromatogram, $210 \mathrm{~nm}$

Peak Quantitation: AREA

Calculation Method: AREA

\begin{tabular}{rrrrr} 
No. & RT & Area & Area \& & BC \\
\hline 1 & 20.120 & 7435128 & 99.774 & BB \\
2 & 21.760 & 16868 & 0.226 & BB \\
\hline & & 7451996 & 100.000 & \\
\hline
\end{tabular}



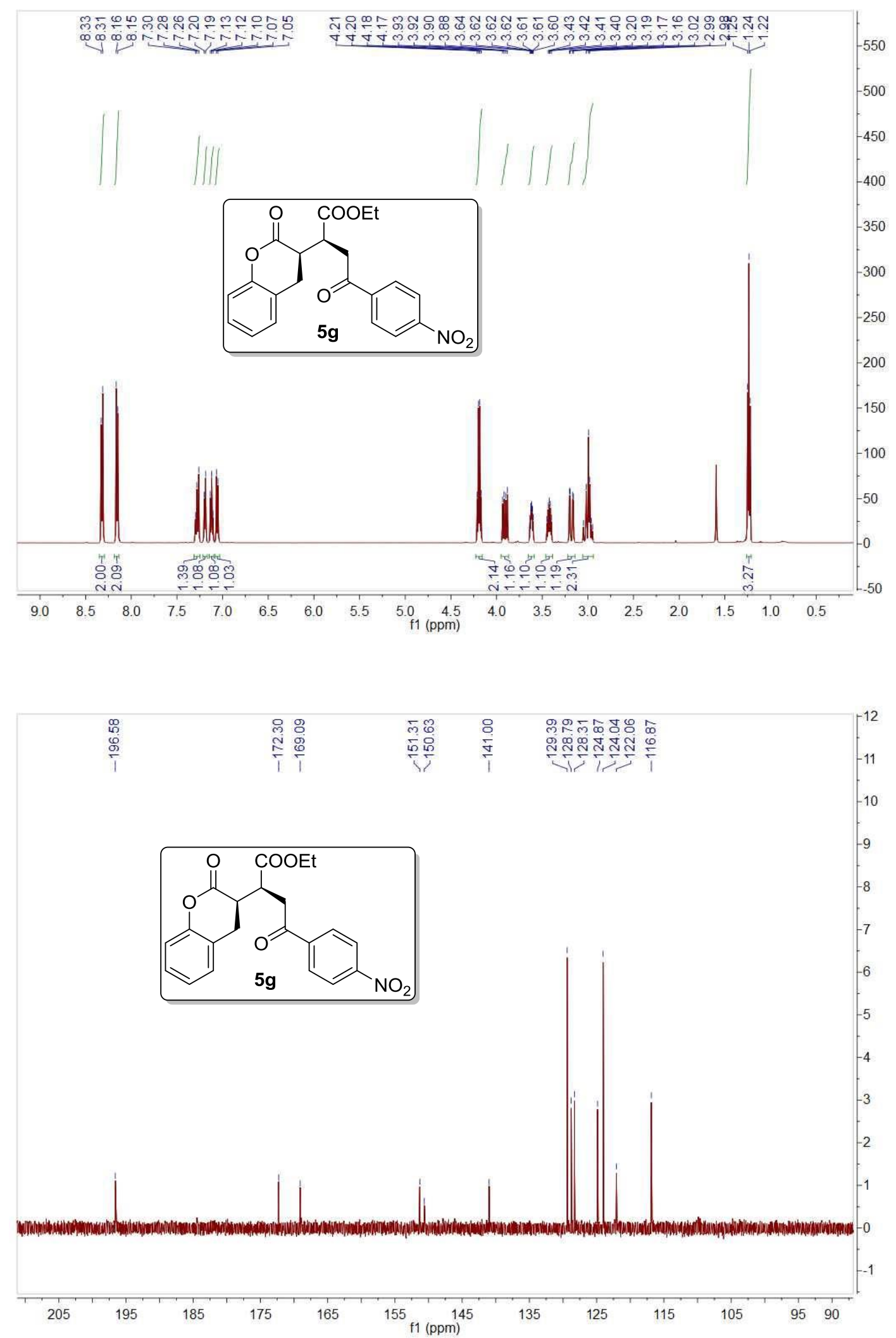


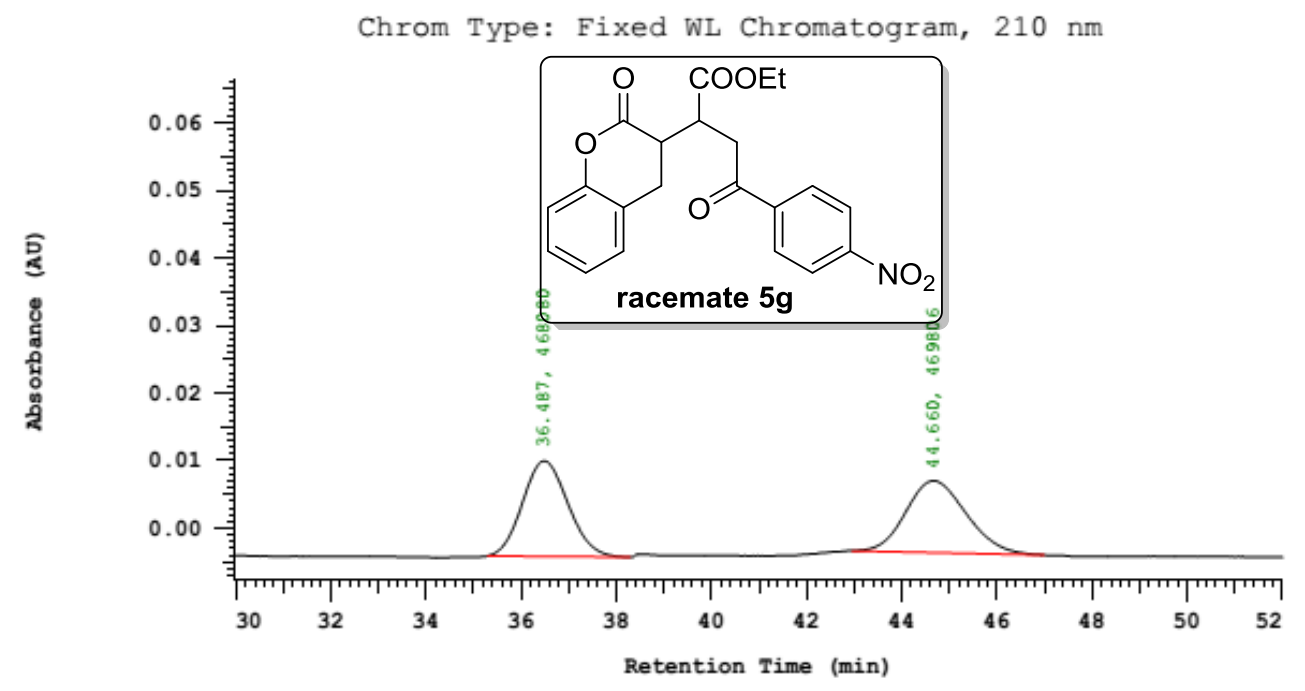

Chrom Type: Fixed WL Chromatogram, $210 \mathrm{~nm}$

Peak Quantitation: AREA

Calculation Method: AREA?

\begin{tabular}{rrrrr} 
No. & RT & Area & Area 8 & BC \\
\hline 1 & 36.487 & 468080 & 49.908 & BB \\
2 & 44.660 & 469806 & 50.092 & BB \\
\hline & 937886 & 100.000 & \\
\hline
\end{tabular}

Chrom Type: Fixed WL Chromatogram, $210 \mathrm{~nm}$

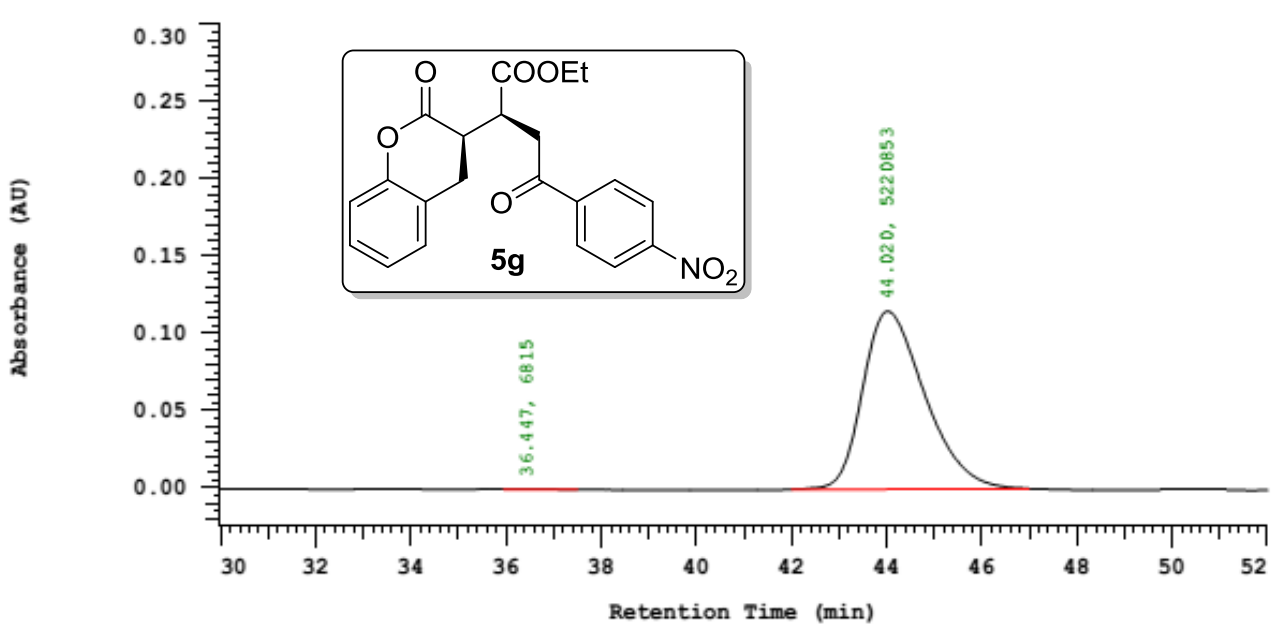

Chrom Type: Fixed WL Chromatogram, $210 \mathrm{~nm}$

Peak Quantitation: AREA

Calculation Method: AREAs

\begin{tabular}{rrrrr} 
No. & RT & Area & Area o & BC \\
\hline 1 & 36.447 & 6815 & 0.130 & BB \\
2 & 44.020 & 5220853 & 99.870 & BB \\
\hline & 5227668 & 100.000 & \\
\hline
\end{tabular}



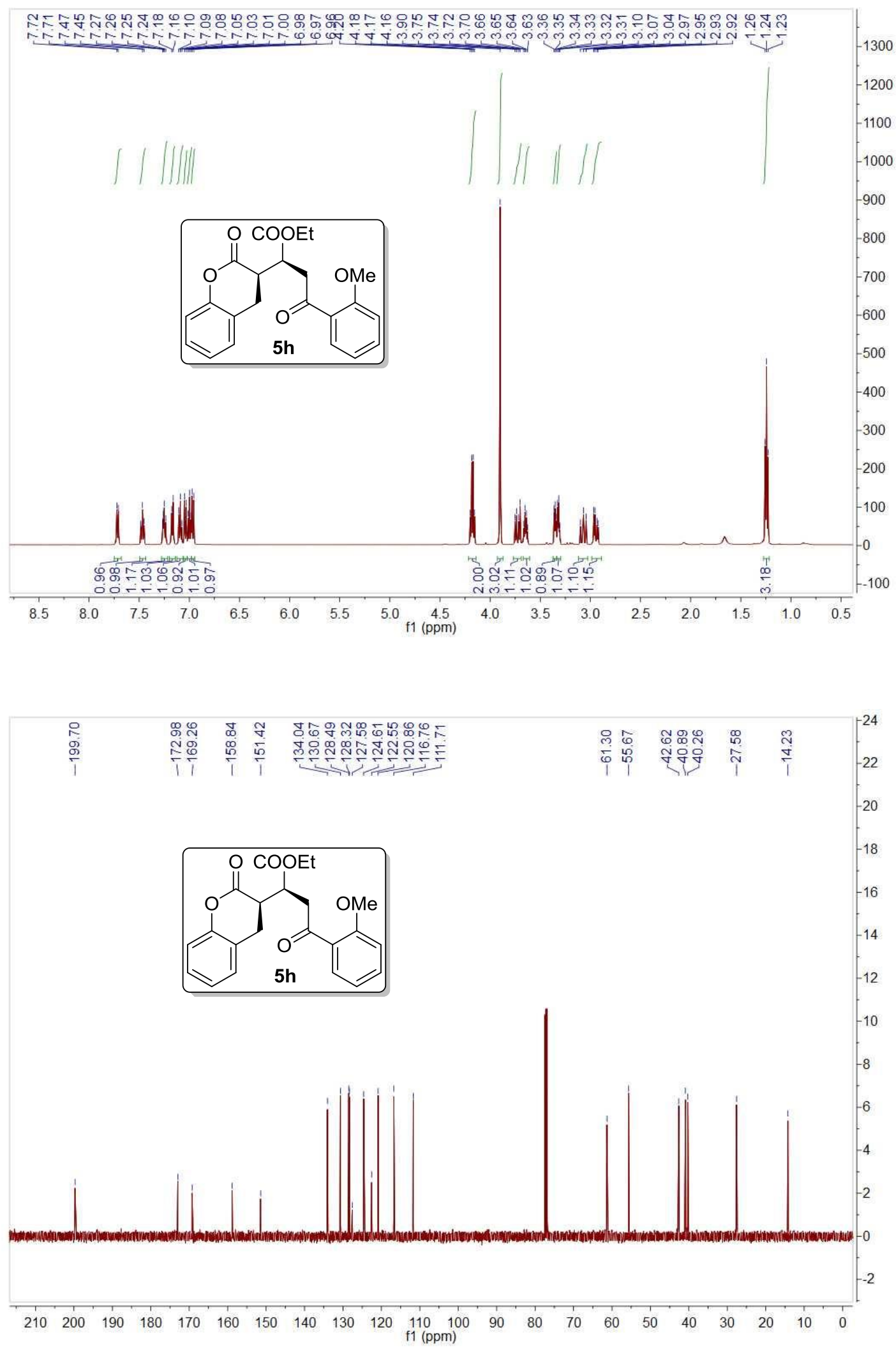
Chrom Type: Fixed WL Chromatogram, $210 \mathrm{~nm}$

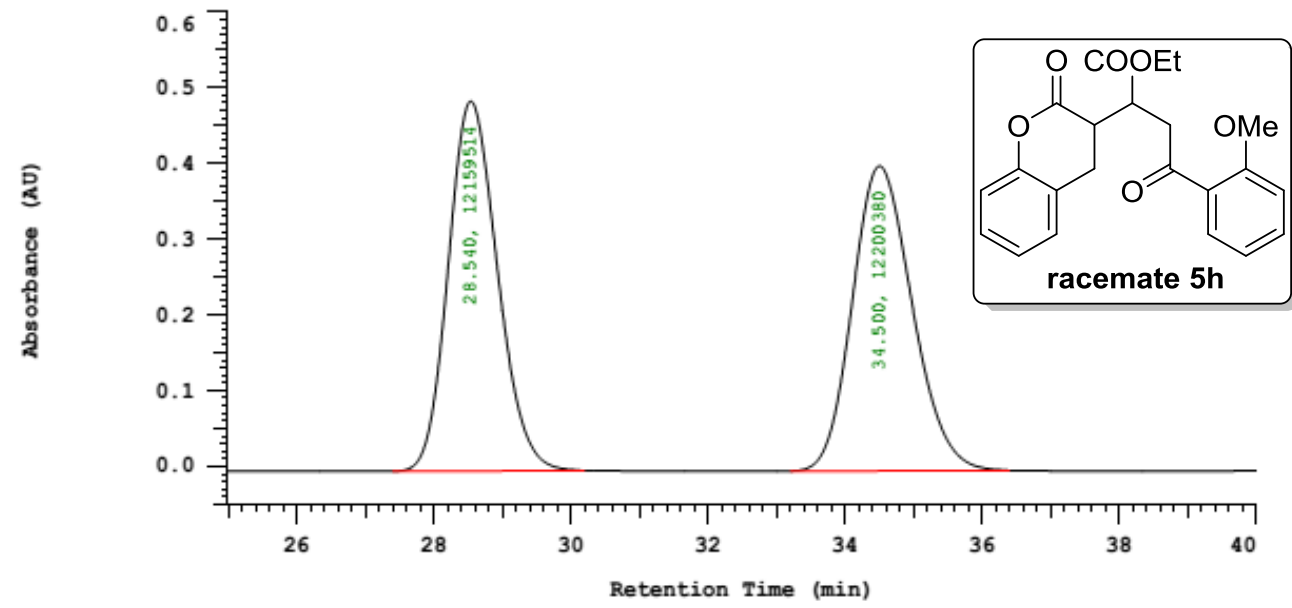

Chrom Type: Fixed WL Chromatogram, $210 \mathrm{~nm}$

Peak Quantitation: AREA

Calculation Method: AREA

\begin{tabular}{ccccc} 
No. & RT & Area & Area & B \\
\hline 1 & 28.540 & 12159514 & 49.916 & BB \\
2 & 34.500 & 12200380 & 50.084 & BB \\
\hline & & 24359894 & 100.000 &
\end{tabular}

Chrom Type: Fixed WL Chromatogram, $210 \mathrm{~nm}$

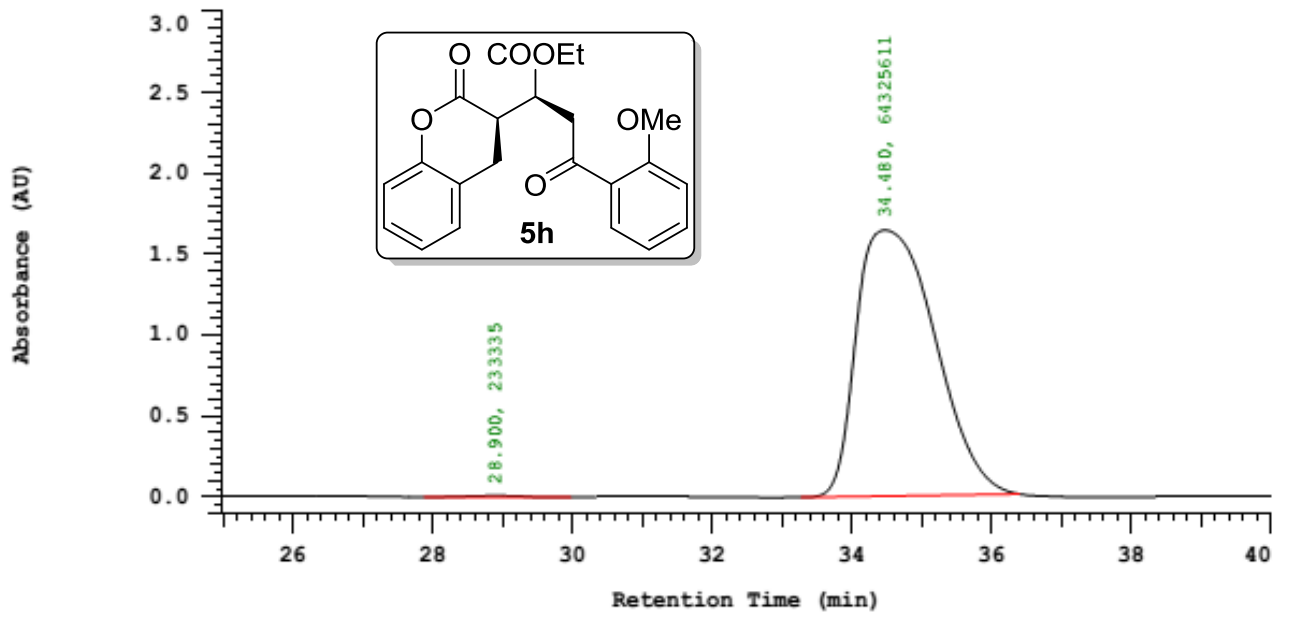

Chrom Type: Fixed WL Chromatogram, $210 \mathrm{~nm}$

Peak Quantitation: AREA

Calculation Method: AREA

\begin{tabular}{crrrr} 
No. & RT & \multicolumn{1}{c}{ Area } & Area 8 & BC \\
\hline 1 & 28.900 & 233335 & 0.361 & BB \\
2 & 34.480 & 64325611 & 99.639 & BB \\
\hline & & 64558946 & 100.000 & \\
\hline
\end{tabular}



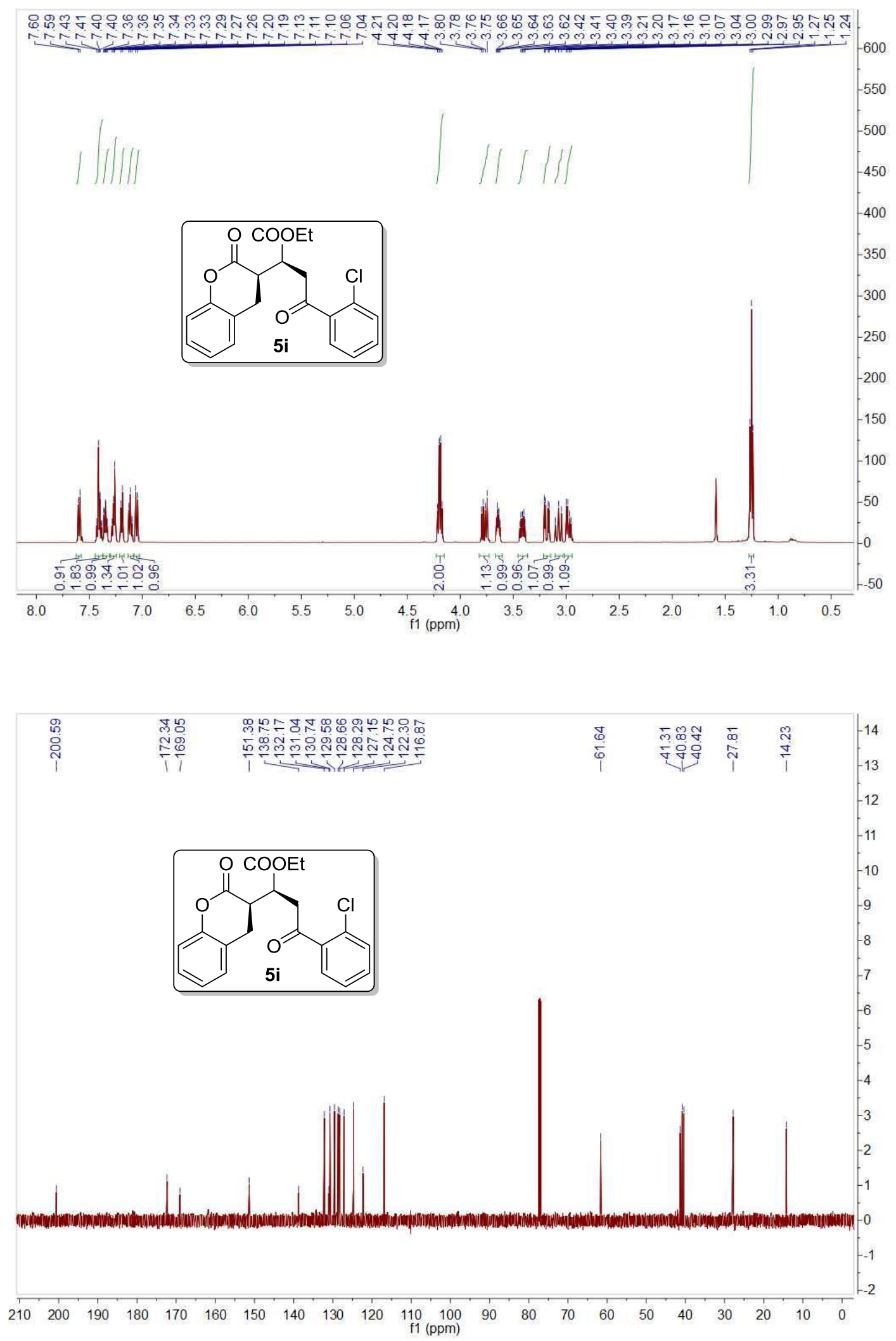
Chrom Type: Fixed WL Chromatogram, $210 \mathrm{~nm}$

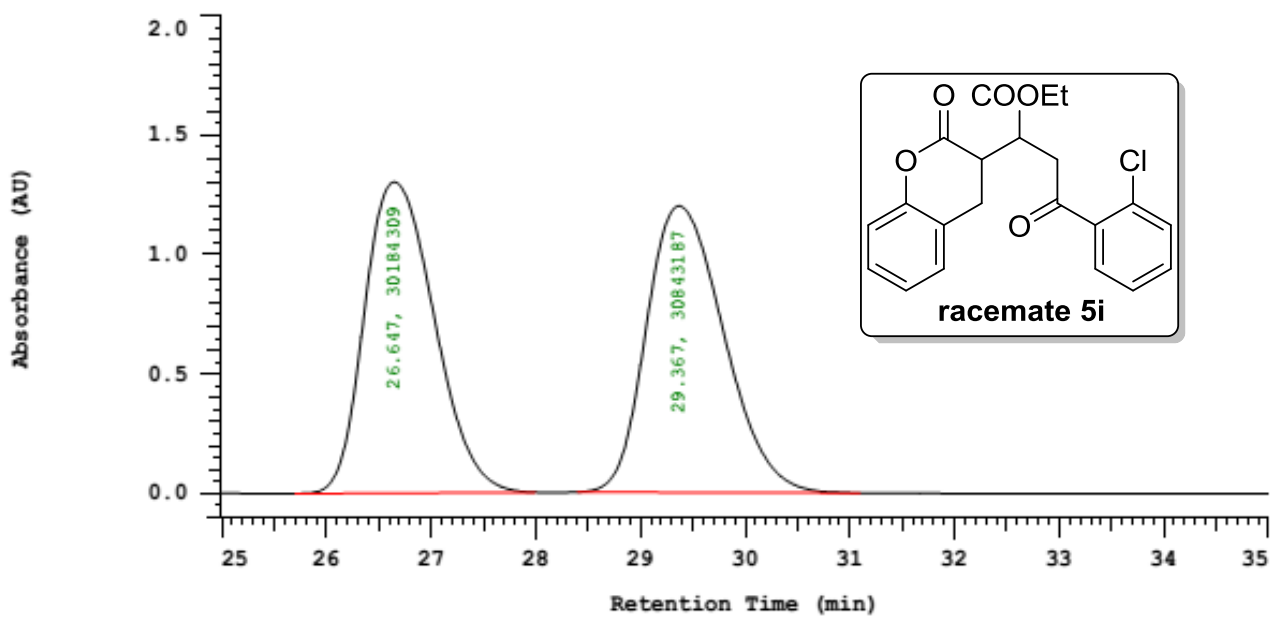

Chrom Type: Fixed WL Chromatogram, 210 nm

Peak Quantitation: AREA

Calculation Method: AREA용

\begin{tabular}{ccccc} 
No. & RT & Area & Area 8 & BC \\
\hline 1 & 26.647 & 30184309 & 49.460 & BB \\
2 & 29.367 & 30843187 & 50.540 & BB \\
\hline & & 61027496 & 100.000 & \\
\hline
\end{tabular}

Chrom Type: Fixed WL Chromatogram, $210 \mathrm{~nm}$

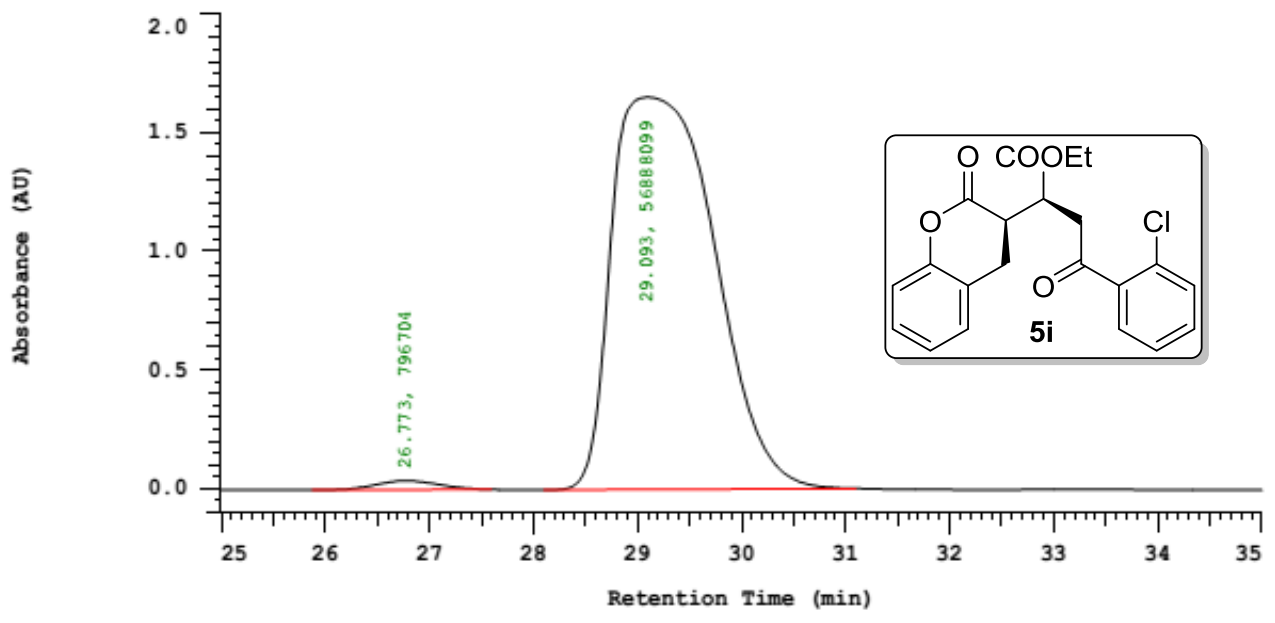

Chrom Type: Fixed WL Chromatogram, $210 \mathrm{~nm}$

Peak Quantitation: AREA

Calculation Method: AREA

\begin{tabular}{rrrrr} 
No. & RT & \multicolumn{1}{c}{ Area } & Area 8 & BC \\
\hline 1 & 26.773 & 796704 & 1.381 & BB \\
2 & 29.093 & 56888099 & 98.619 & BB \\
\hline & 57684803 & 100.000 & \\
\hline
\end{tabular}



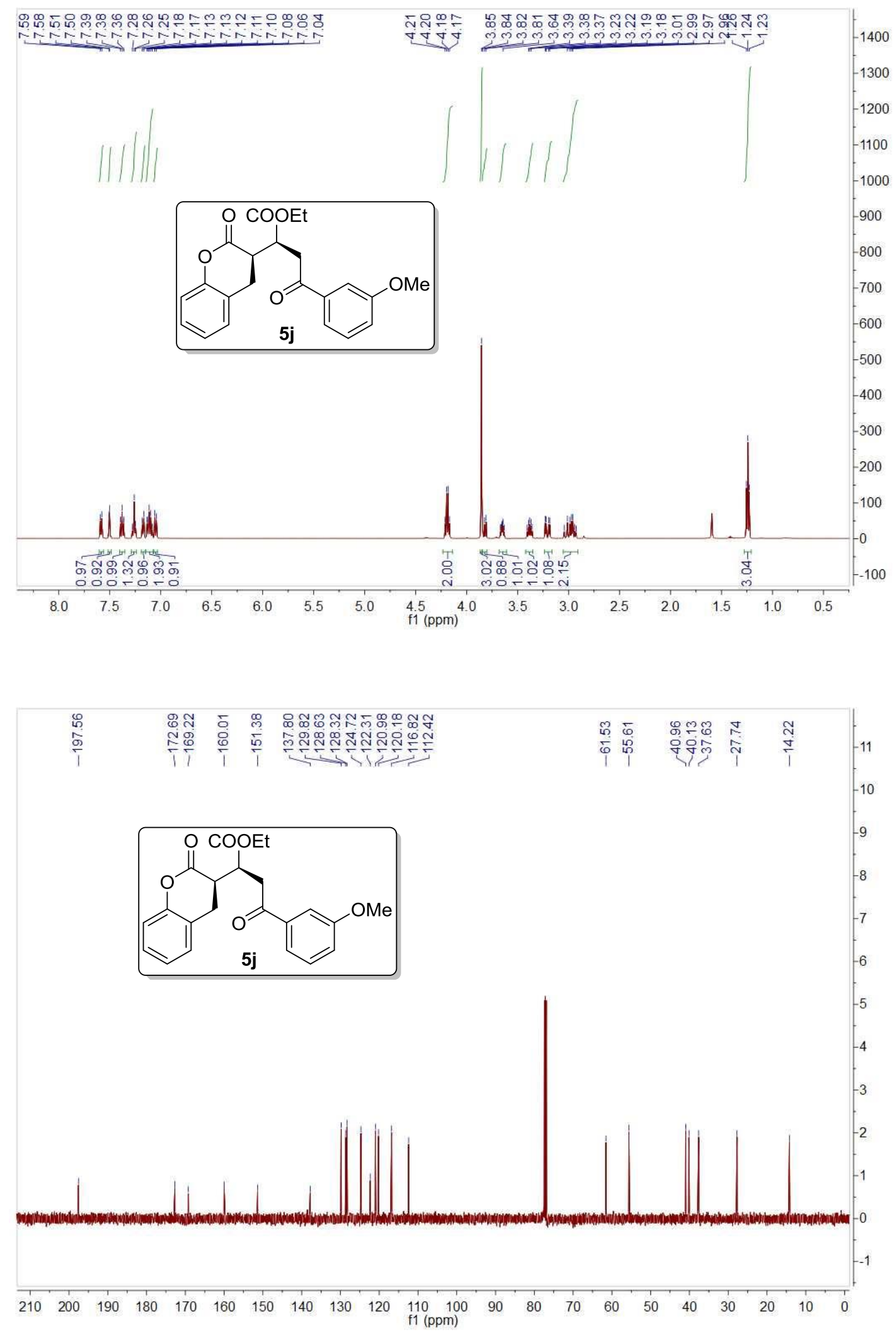
Chrom Type: Fixed WL Chromatogram, $210 \mathrm{~nm}$

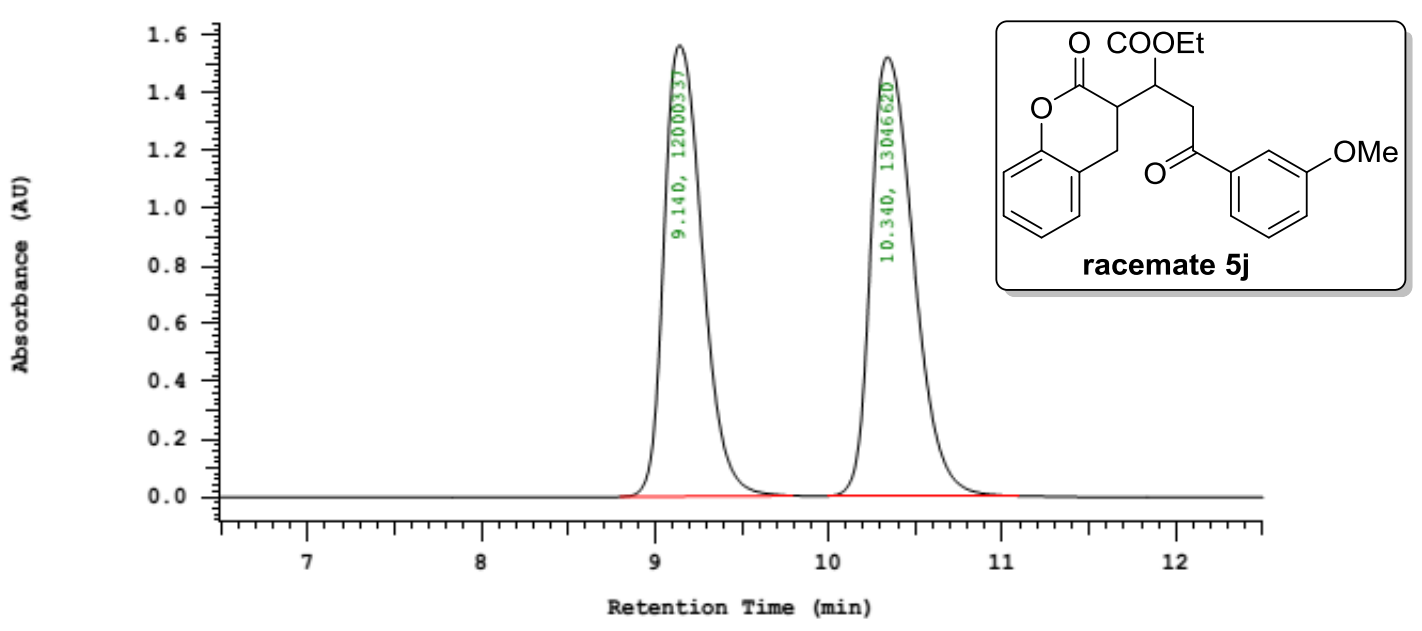

Chrom Type: Fixed WL Chromatogram, $210 \mathrm{~nm}$

Peak Quantitation: AREA

Calculation Method: AREA

\begin{tabular}{rrrrr} 
No. & \multicolumn{1}{c}{ RT } & Area & Area 8 & BC \\
\hline 1 & 9.140 & 12000337 & 47.911 & BB \\
2 & 10.340 & 13046620 & 52.089 & BB \\
\hline & & 25046957 & 100.000 &
\end{tabular}

Chrom Type: Fixed WL Chromatogram, $210 \mathrm{~nm}$

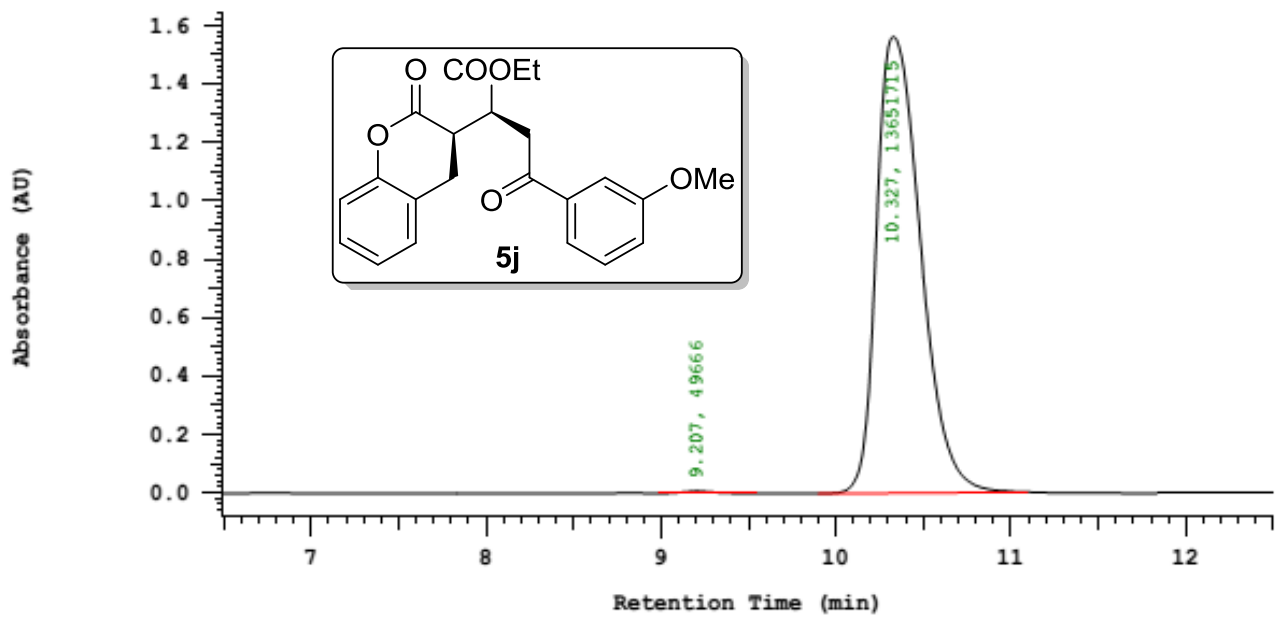

Chrom Type: Fixed WL Chromatogram, $210 \mathrm{~nm}$

Peak Quantitation: AREA

Calculation Method: AREA\&

\begin{tabular}{|c|c|c|c|c|}
\hline No. & $\mathrm{RT}$ & Area & Area \& & $\mathrm{BC}$ \\
\hline 1 & 9.207 & 49666 & 0.362 & $\mathrm{BB}$ \\
\hline \multirow[t]{2}{*}{2} & 10.327 & 13651715 & 99.638 & $\mathrm{BB}$ \\
\hline & & 13701381 & 100.000 & \\
\hline
\end{tabular}



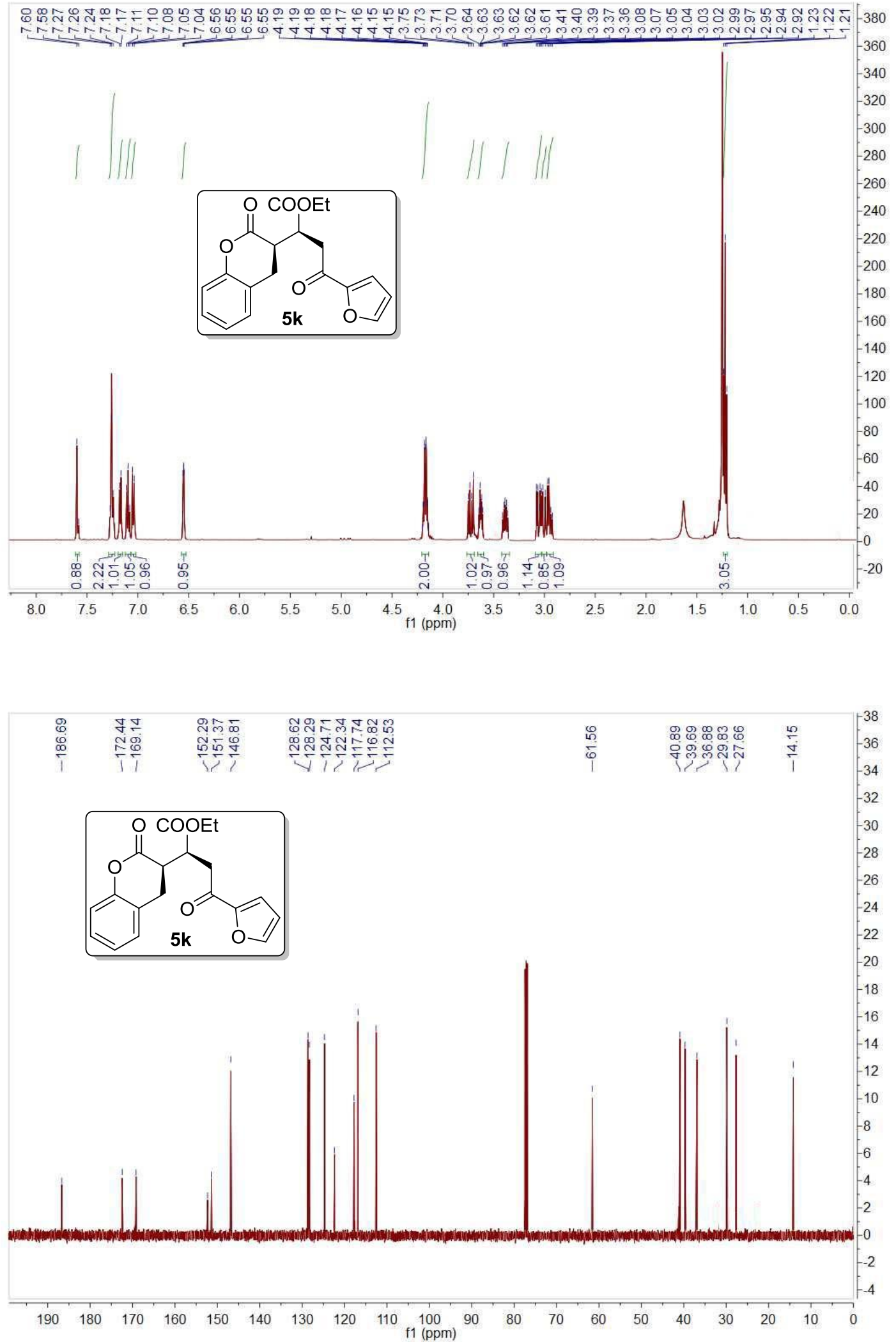


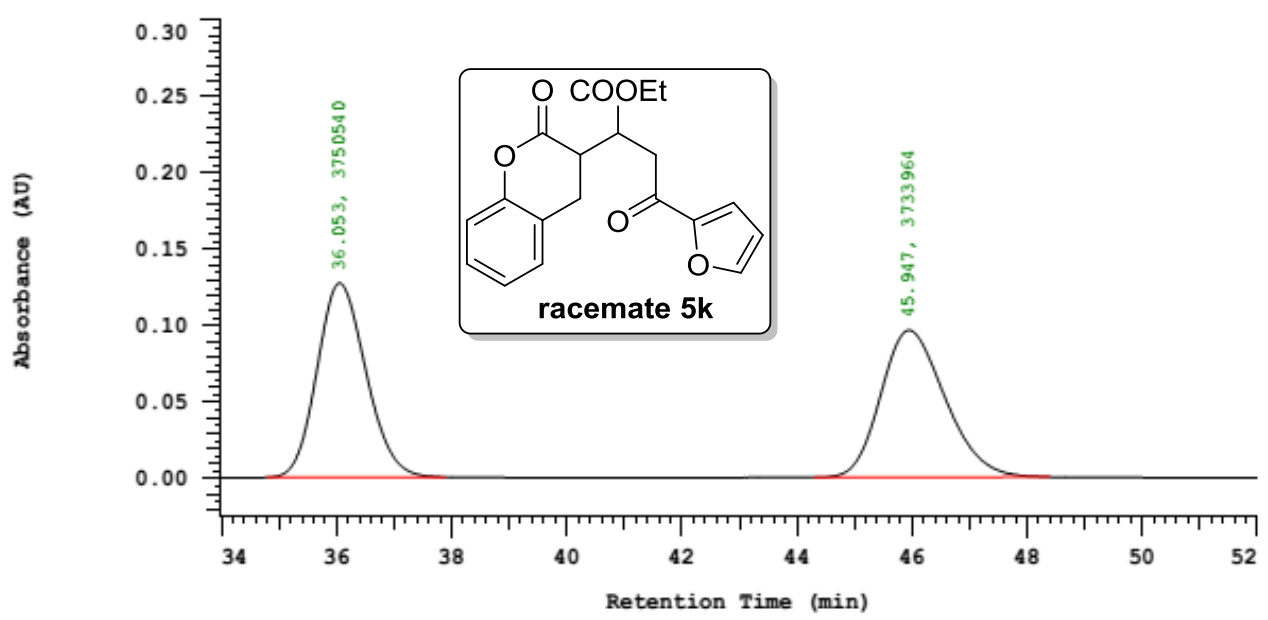

Peak Quantitation: AREA

Calculation Method: AREA\&

\begin{tabular}{ccccc} 
No. & RT & Area & Area 8 & BC \\
\hline 1 & 36.053 & 3750540 & 50.111 & BB \\
2 & 45.947 & 3733964 & 49.889 & BB \\
\hline & & 7484504 & 100.000 & \\
\hline
\end{tabular}

Chrom Type: Fixed WL Chromatogram, $210 \mathrm{~nm}$

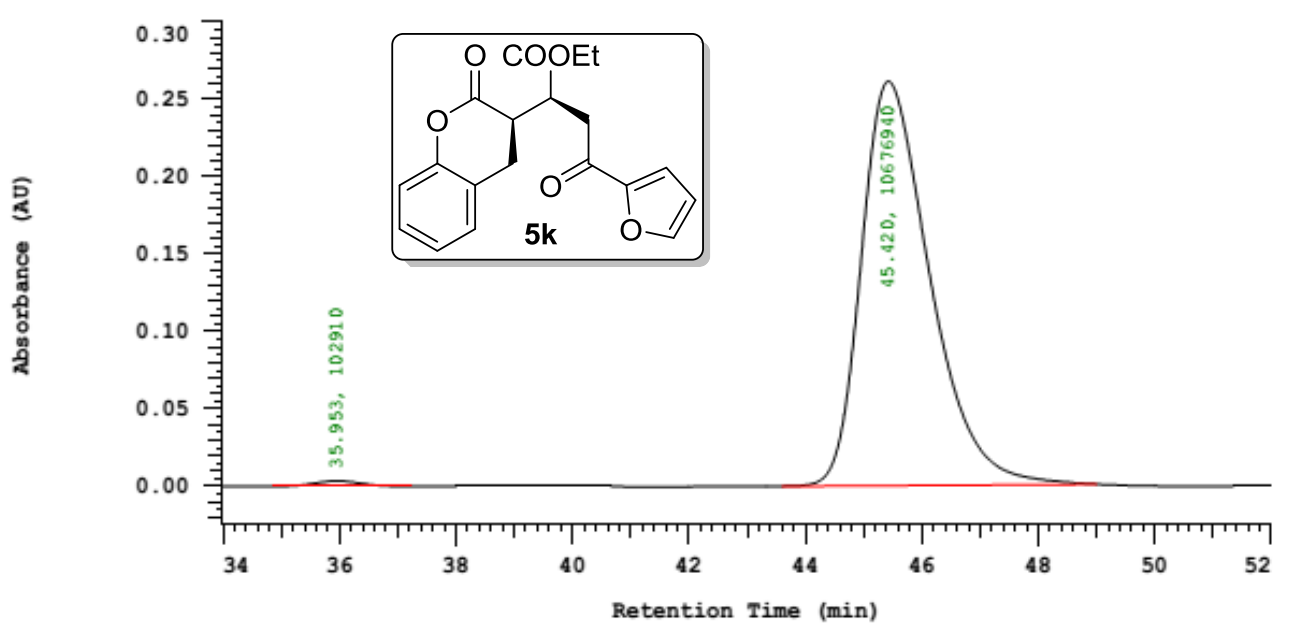

Chrom Type: Fixed WL Chromatogram, $210 \mathrm{~nm}$

Peak Quantitation: AREA

Calculation Method: AREA?

\begin{tabular}{rrrrr} 
No. & RT & \multicolumn{1}{c}{ Area } & Area \& & BC \\
\hline 1 & 35.953 & 102910 & 0.955 & BB \\
2 & 45.420 & 10676940 & 99.045 & BB \\
\hline & 10779850 & 100.000 & \\
\hline
\end{tabular}



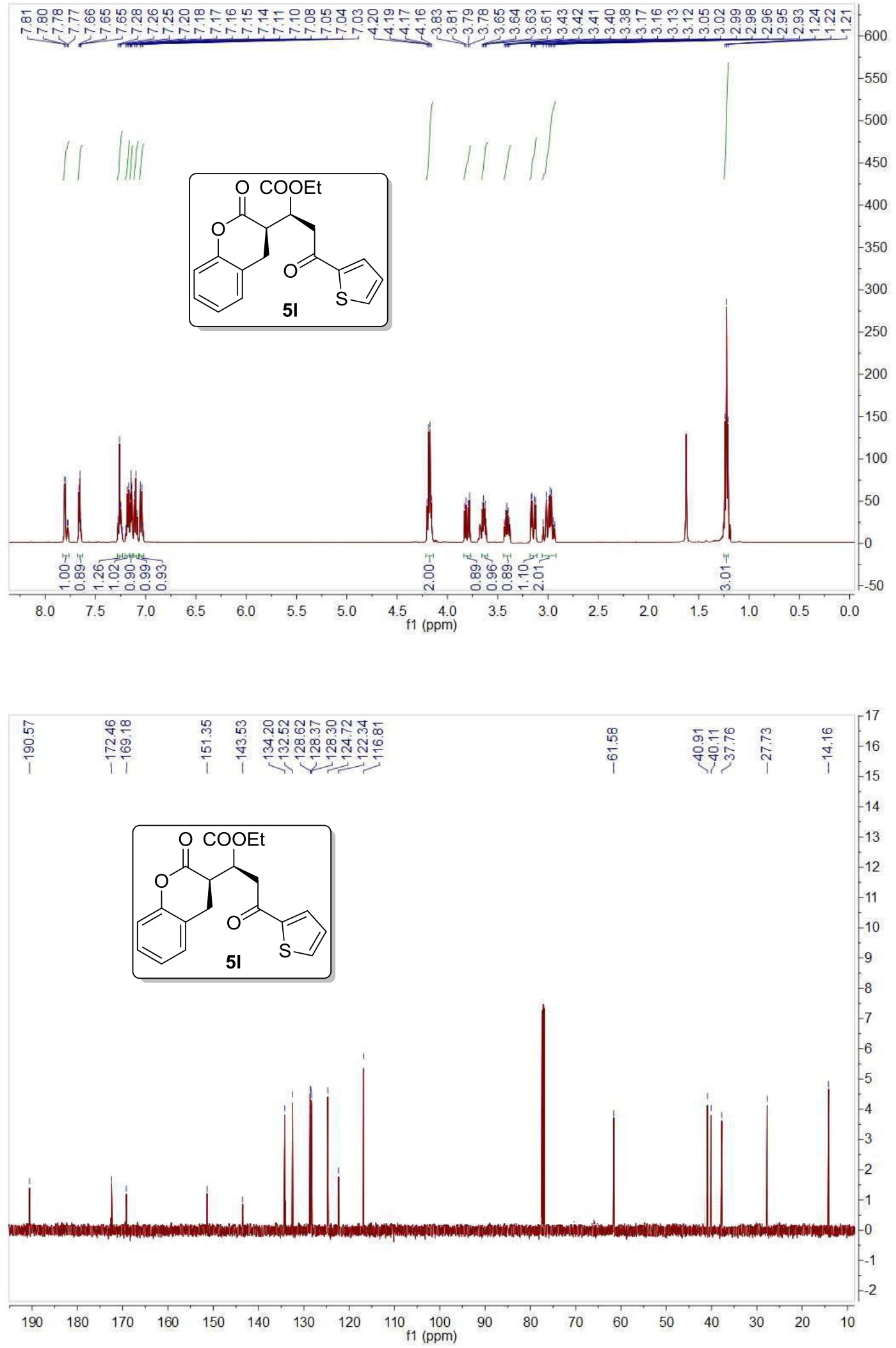
Chrom Type: Fixed WL Chromatogram, $210 \mathrm{~nm}$

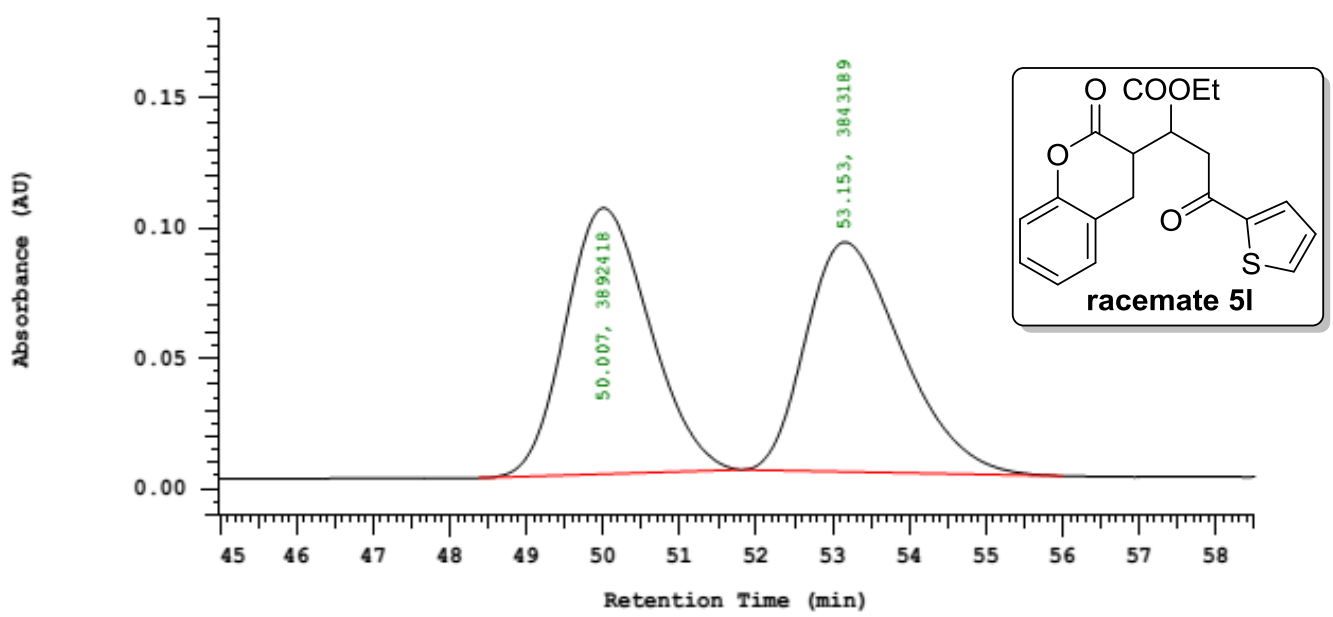

Chrom Type: Fixed WL Chromatogram, $210 \mathrm{~nm}$

Peak Quantitation: AREA

Calculation Method: AREA\&

\begin{tabular}{ccccc} 
No. & RT & Area & Area \& & BC \\
\hline 1 & 50.007 & 3892418 & 50.318 & BB \\
2 & 53.153 & 3843189 & 49.682 & BB \\
\hline & 7735607 & 100.000 & \\
\hline
\end{tabular}

Chrom Type: Fixed WL Chromatogram, $210 \mathrm{~nm}$

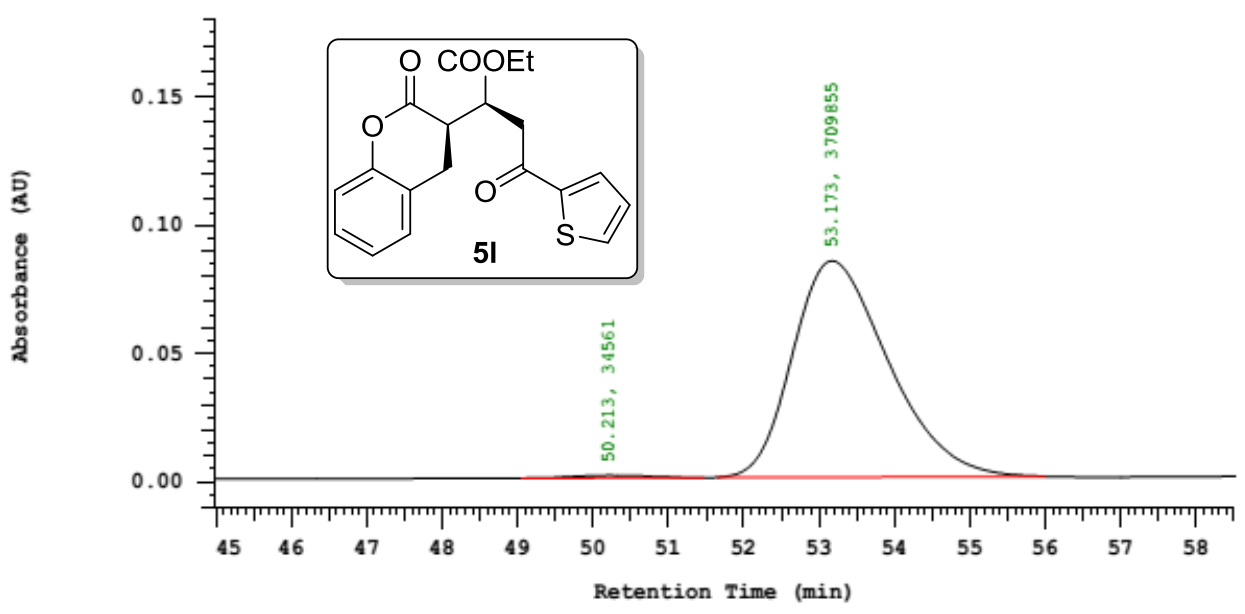

Chrom Type: Fixed WL Chromatogram, $210 \mathrm{~nm}$

Peak Quantitation: AREA

Calculation Method: AREA요

\begin{tabular}{rrrrr} 
No. & RT & Area & Area o & BC \\
\hline 1 & 50.213 & 34561 & 0.923 & BB \\
2 & 53.173 & 3709855 & 99.077 & BB \\
\hline & 3744416 & 100.000 & \\
\hline
\end{tabular}



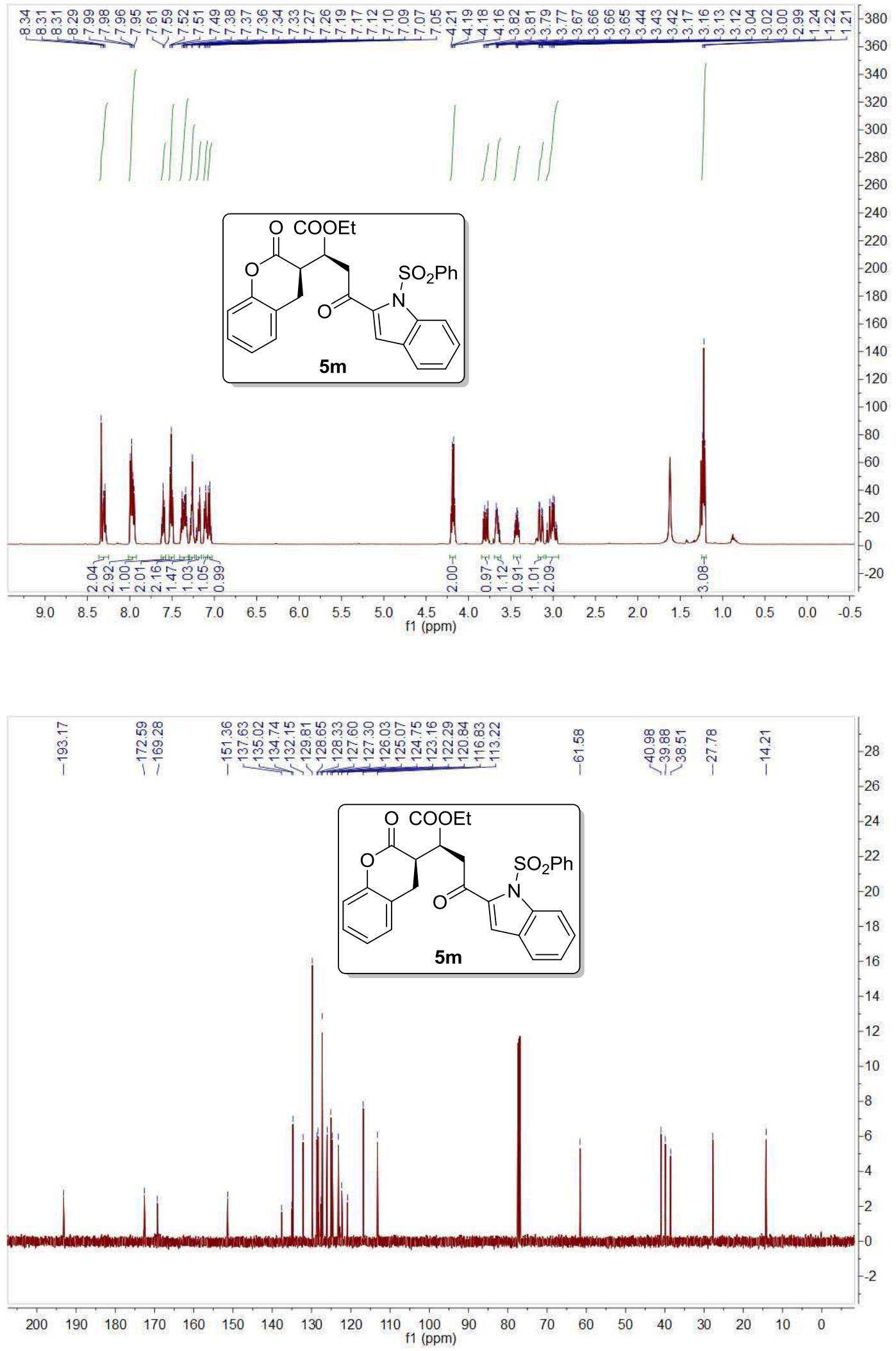
Chrom Type: Fixed WL Chromatogram, $210 \mathrm{~nm}$

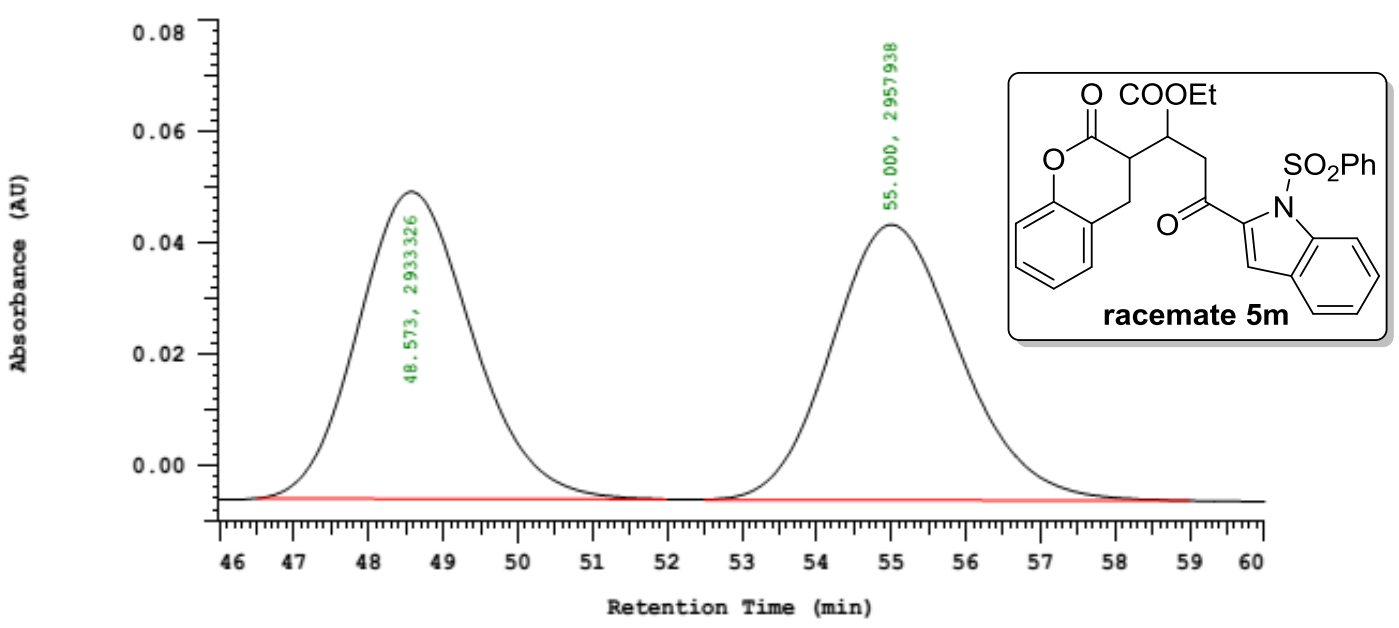

Peak Quantitation: AREA

Calculation Method: AREA\&

\begin{tabular}{ccccc} 
No. & RT & Area & Area \& & BC \\
\hline 1 & 48.573 & 2933326 & 49.791 & BB \\
2 & 55.000 & 2957938 & 50.209 & BB \\
\hline & & 5891264 & 100.000 & \\
\hline
\end{tabular}

Chrom Type: Fixed WL Chromatogram, $210 \mathrm{~nm}$

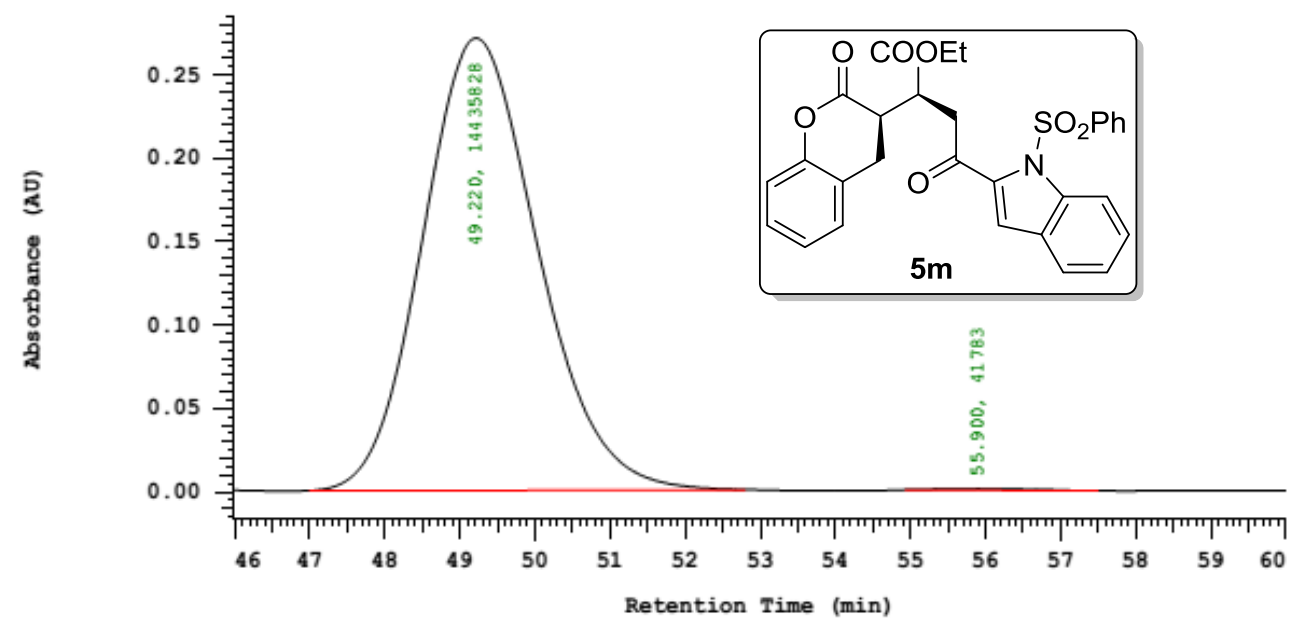

Chrom Type: Fixed WL Chromatogram, $210 \mathrm{~nm}$

Peak Quantitation: AREA

Calculation Method: AREA응

\begin{tabular}{rrrrr} 
No. & RT & \multicolumn{1}{c}{ Area } & Area o & BC \\
\hline 1 & 49.220 & 14435828 & 99.711 & BB \\
2 & 55.900 & 41783 & 0.289 & BB \\
\hline & & 14477611 & 100.000 & \\
\hline
\end{tabular}



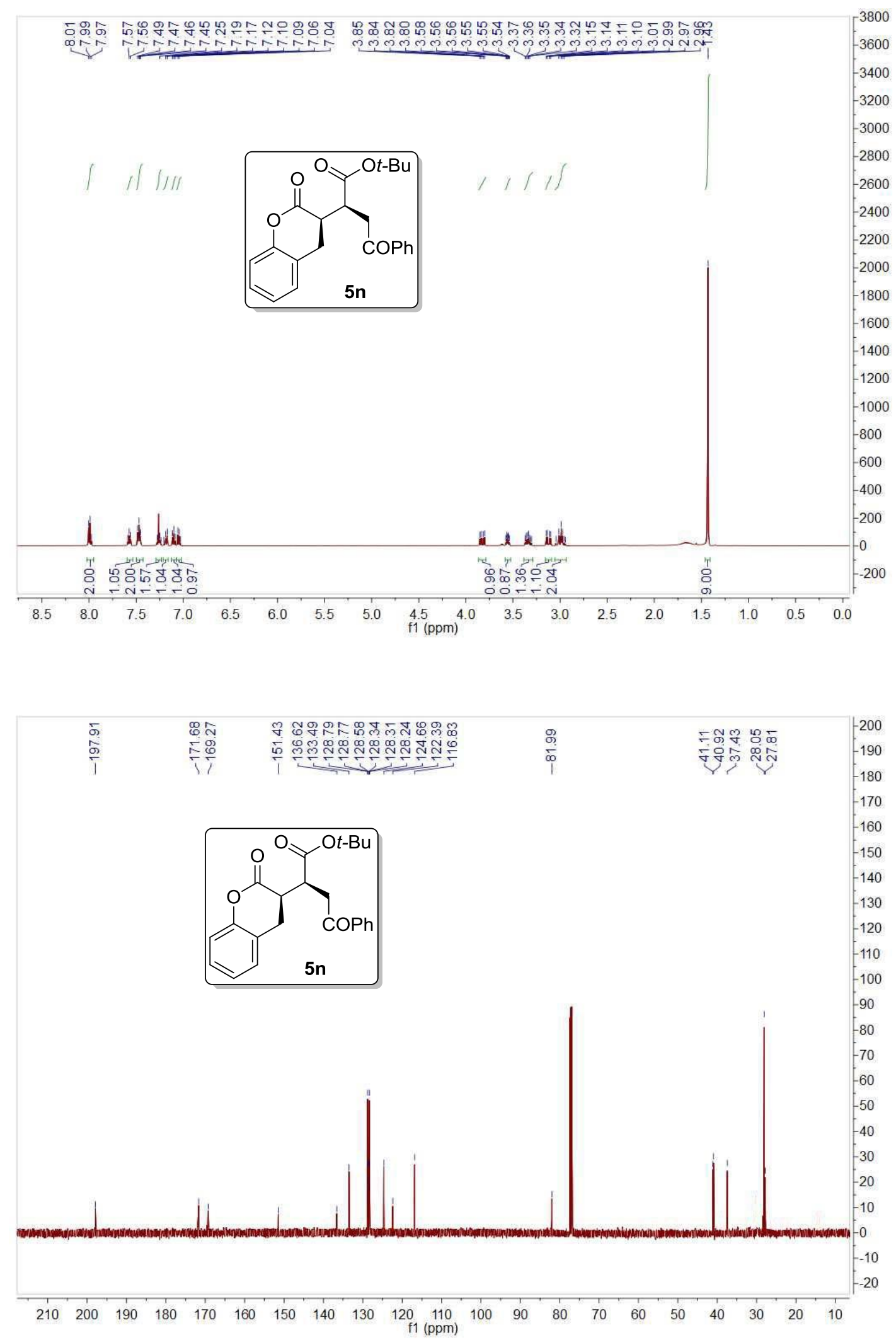
Chrom Type: Fixed WL Chromatogram, $210 \mathrm{~nm}$

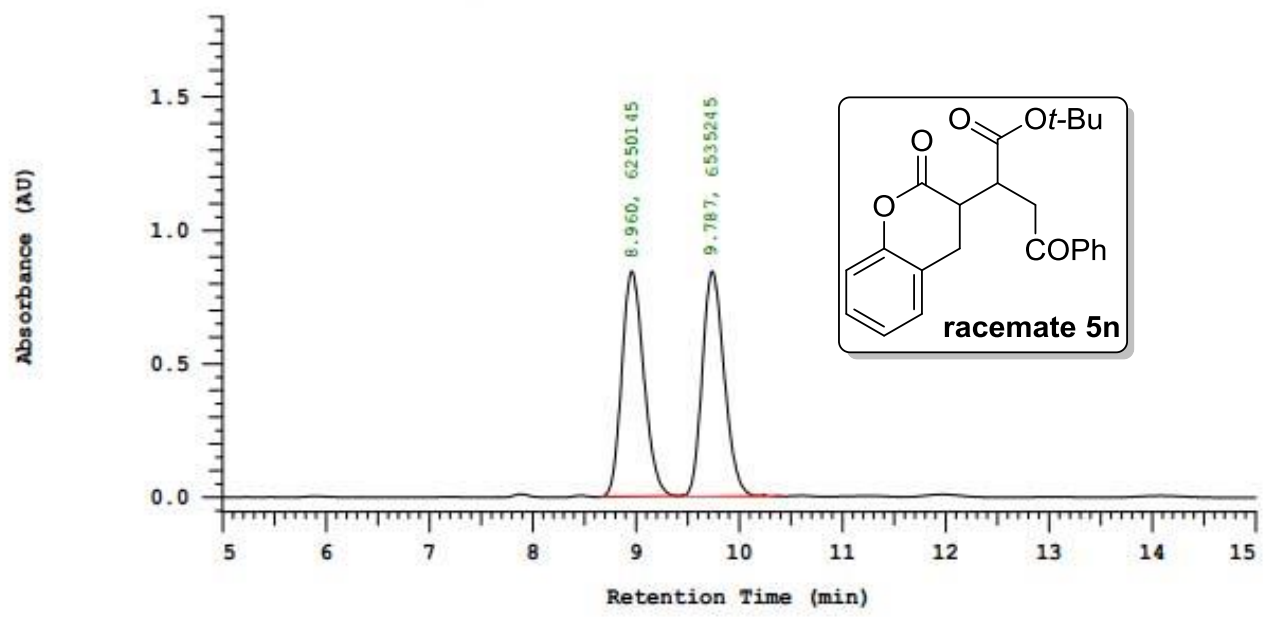

Chrom Type: Fixed WL Chromatogram, $210 \mathrm{~nm}$

Peak Quantitation: AREA

Calculation Method: AREA응

\begin{tabular}{ccccc} 
No. & RT & Area & Area \& & BC \\
\hline 1 & 8.960 & 6250145 & 48.885 & BB \\
2 & 9.787 & 6535245 & 51.115 & BB \\
\hline & & 12785390 & 100.000 & \\
\hline
\end{tabular}

Chrom Type: Fixed WL Chromatogram, $210 \mathrm{~nm}$

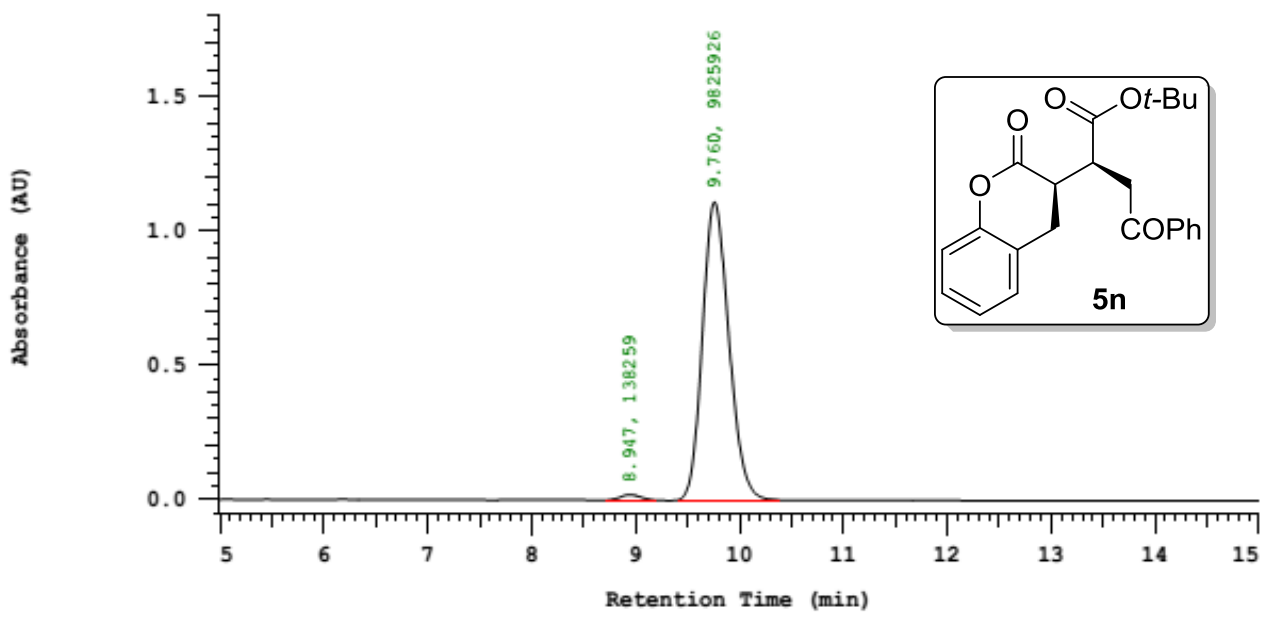

Chrom Type: Fixed WL Chromatogram, $210 \mathrm{~nm}$

Peak Quantitation: AREA

Calculation Method: AREA

\begin{tabular}{ccccc} 
No. & RT & Area & Area o & BC \\
\hline 1 & 8.947 & 138259 & 1.388 & BB \\
2 & 9.760 & 9825926 & 98.612 & BB \\
\hline & & 9964185 & 100.000 & \\
\hline
\end{tabular}



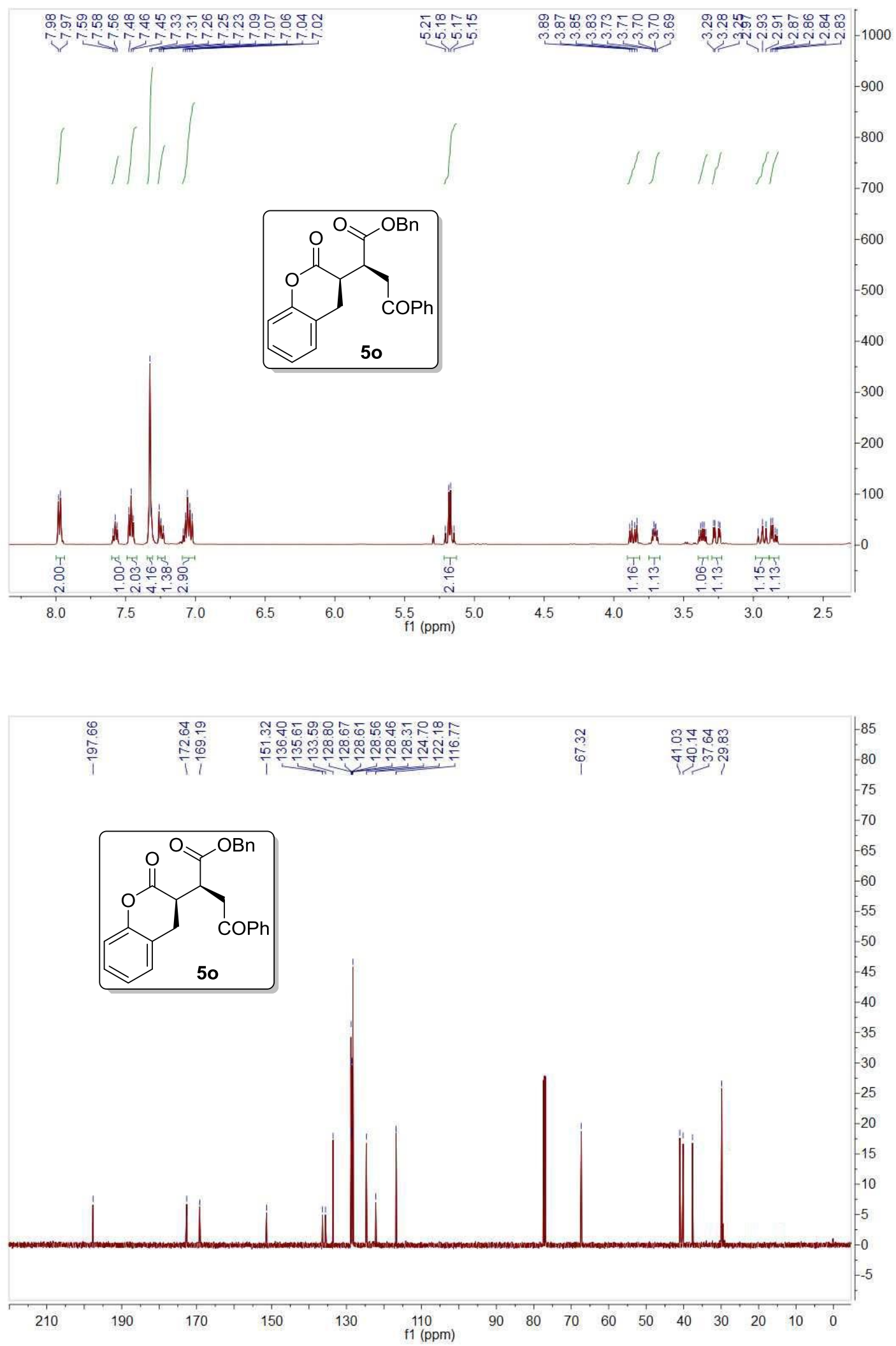
Chrom Type: Fixed WL Chromatogram, $210 \mathrm{~nm}$

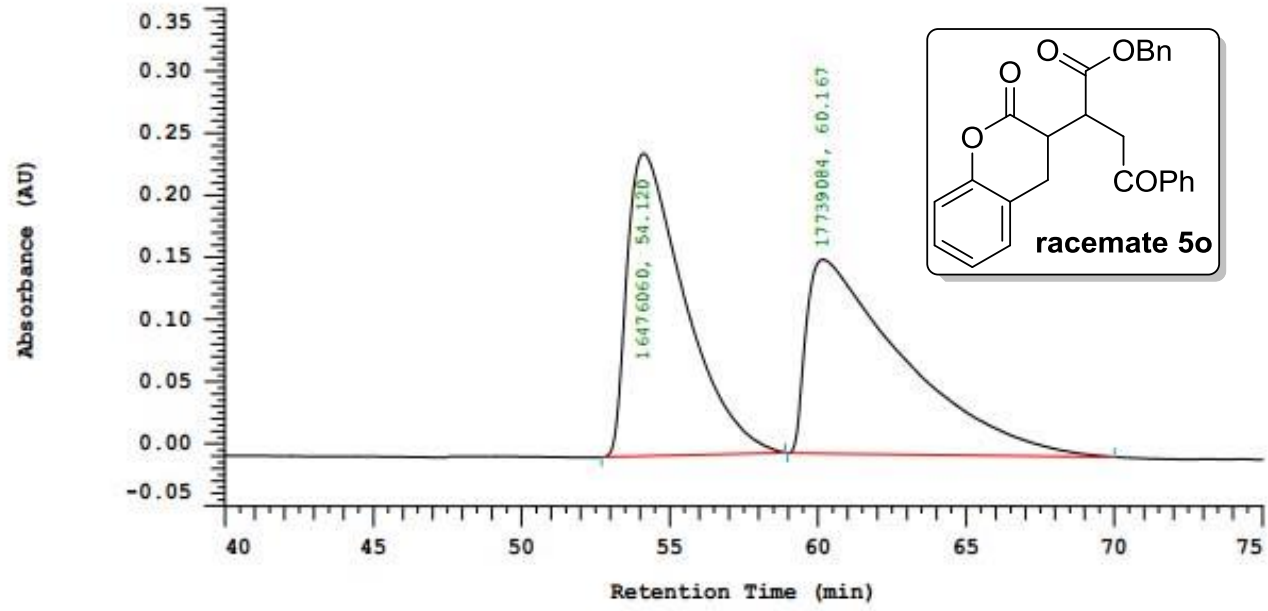

Chrom Type: Fixed WL Chromatogram, $210 \mathrm{~nm}$

Peak Quantitation: AREA

Calculation Method: AREAr

\begin{tabular}{ccccc} 
No. & RT & Area & Area 8 & BC \\
\hline 1 & 54.120 & 16476060 & 48.154 & BB \\
2 & 60.167 & 17739084 & 51.846 & BB \\
\hline & & 34215144 & 100.000 & \\
\hline
\end{tabular}

Chrom Type: Fixed WL Chromatogram, $210 \mathrm{~nm}$

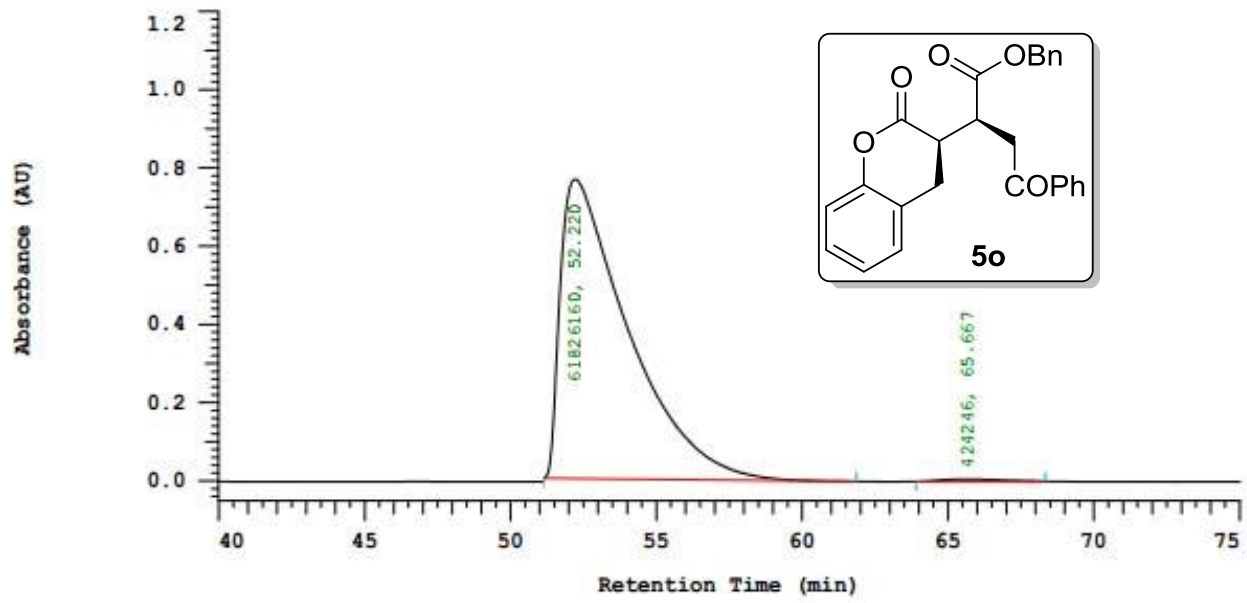

Chrom Type: Fixed WL Chromatogram, $210 \mathrm{~nm}$

Peak Quantitation: AREA

Calculation Method: AREA

\begin{tabular}{rrrrr} 
No. & RT & \multicolumn{1}{c}{ Area } & Area o & BC \\
\hline 1 & 52.220 & 61826160 & 99.318 & BB \\
2 & 65.667 & 424246 & 0.682 & BB \\
\hline & & 62250406 & 100.000 & \\
\hline
\end{tabular}



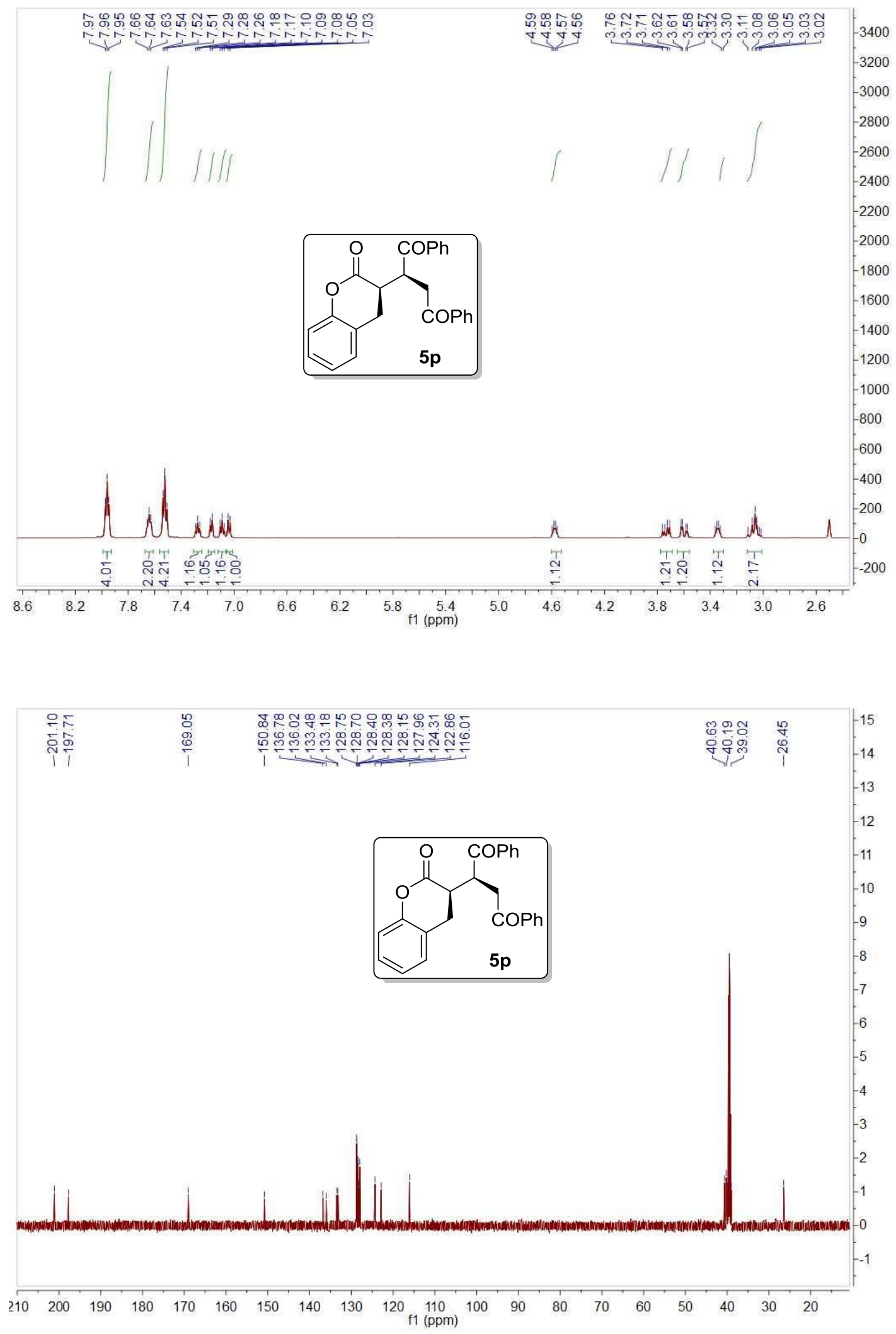
Chrom Type: Fixed WL Chromatogram, 210 nm

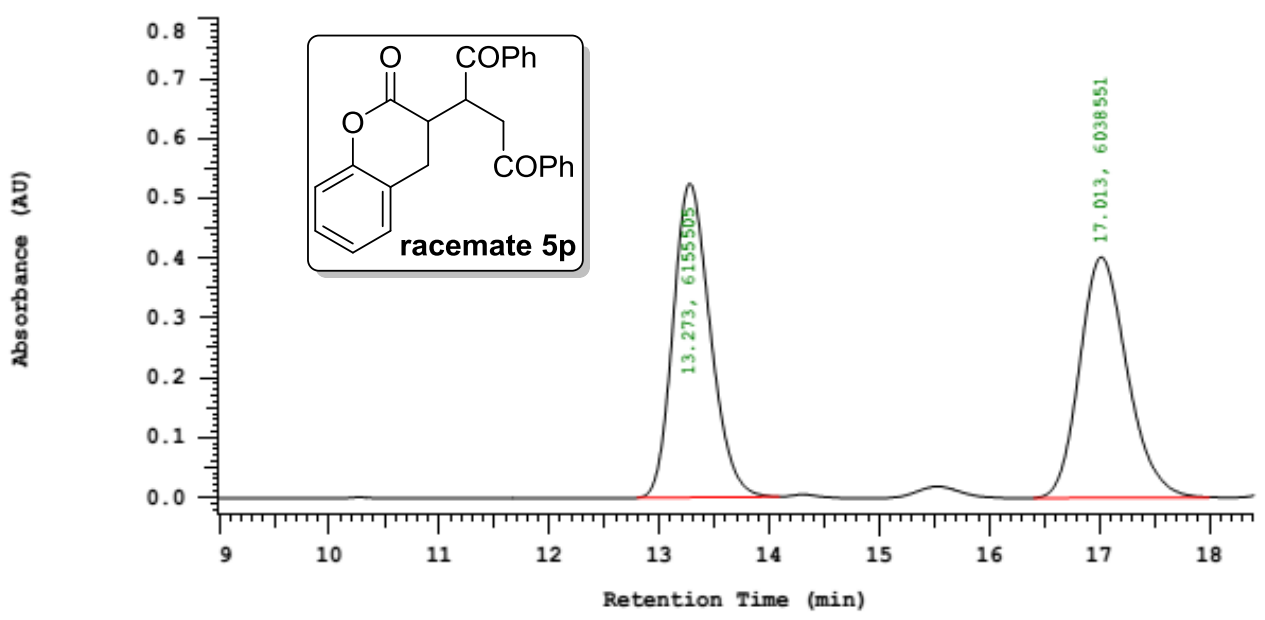

Chrom Type: Fixed WL Chromatogram, $210 \mathrm{~nm}$

Peak Quantitation: AREA

Calculation Method: AREA

\begin{tabular}{ccccc} 
No. & RT & Area & Area o & BC \\
\hline 1 & 13.273 & 6155505 & 50.480 & BB \\
2 & 17.013 & 6038551 & 49.520 & BB \\
\hline & & 12194056 & 100.000 & \\
\hline
\end{tabular}

Chrom Type: Fixed WL Chromatogram, $210 \mathrm{~nm}$

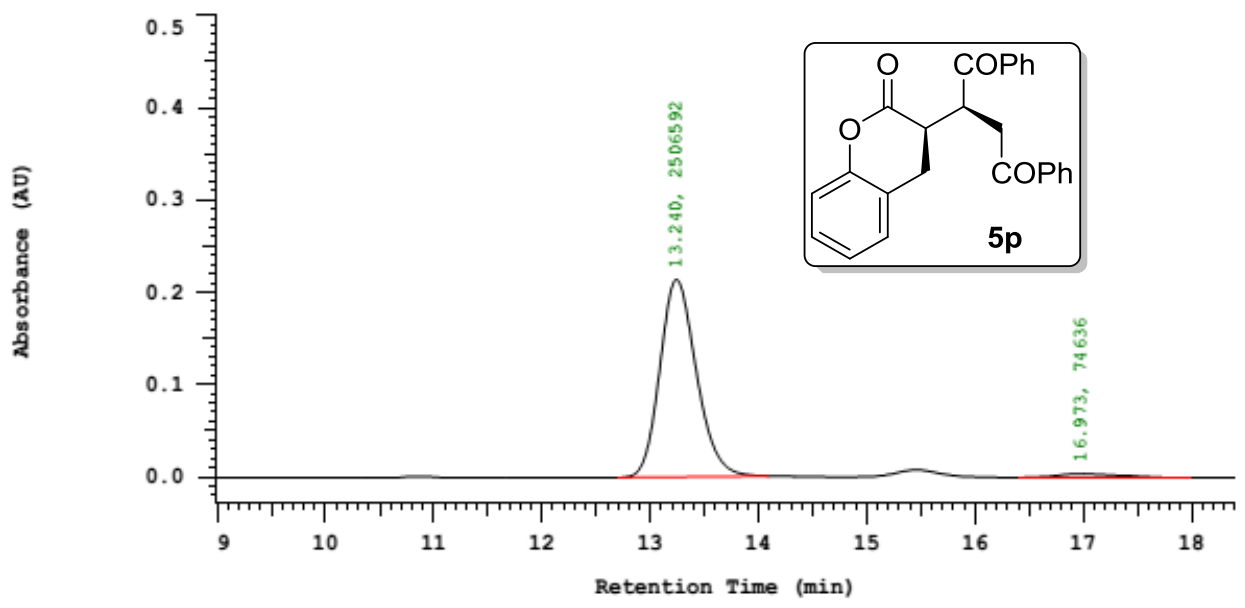

Chrom Type: Fixed WL Chromatogram, $210 \mathrm{~nm}$

Peak Quantitation: AREA

Calculation Method: AREAs

\begin{tabular}{rrrrr} 
No. & RT & \multicolumn{1}{c}{ Area } & Area o & BC \\
\hline 1 & 13.240 & 2506592 & 97.108 & BB \\
2 & 16.973 & 74636 & 2.892 & BB \\
\hline & 2581228 & 100.000 & \\
\hline
\end{tabular}



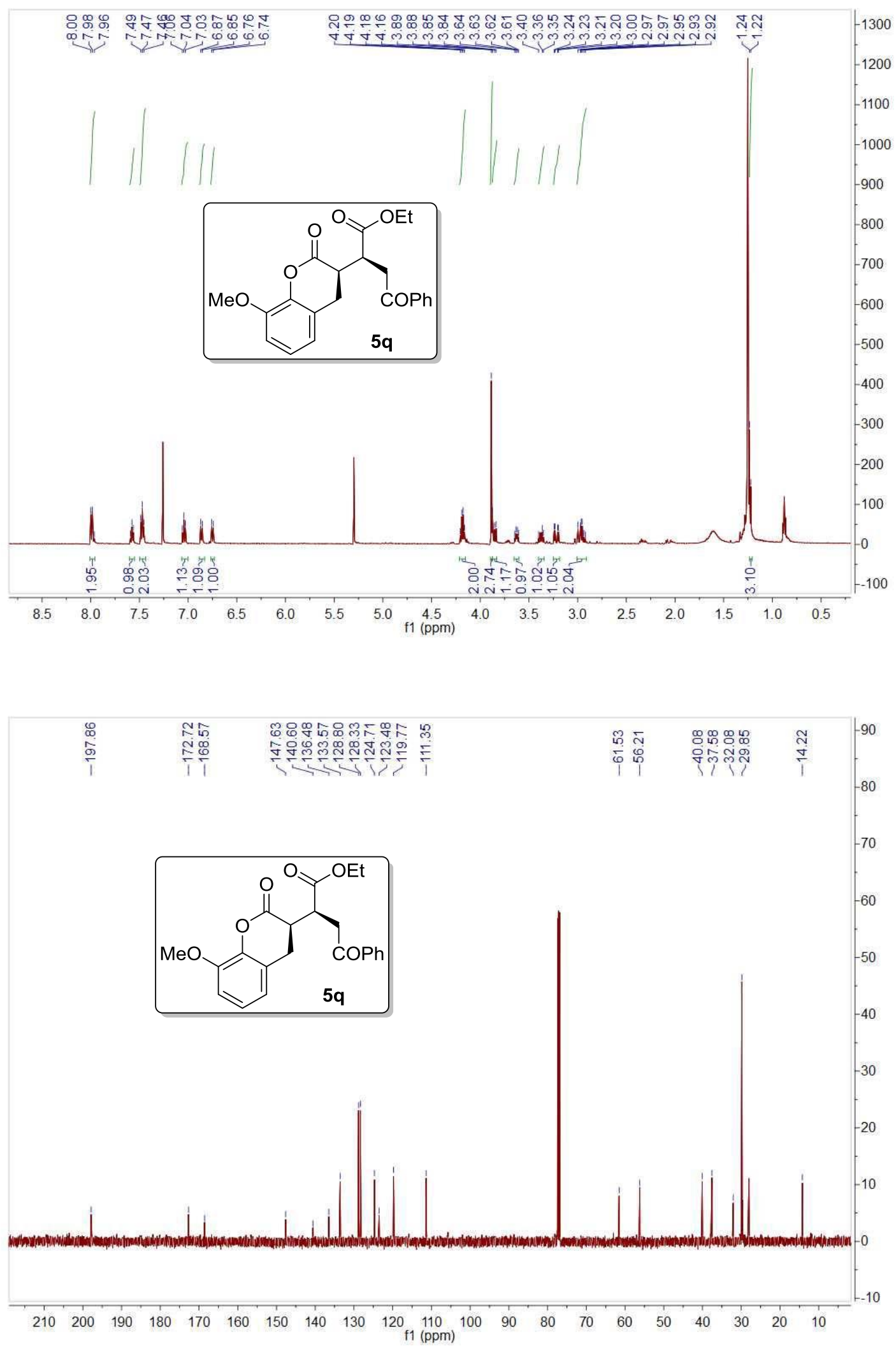
Chrom Type: Fixed WL Chromatogram, $210 \mathrm{~nm}$

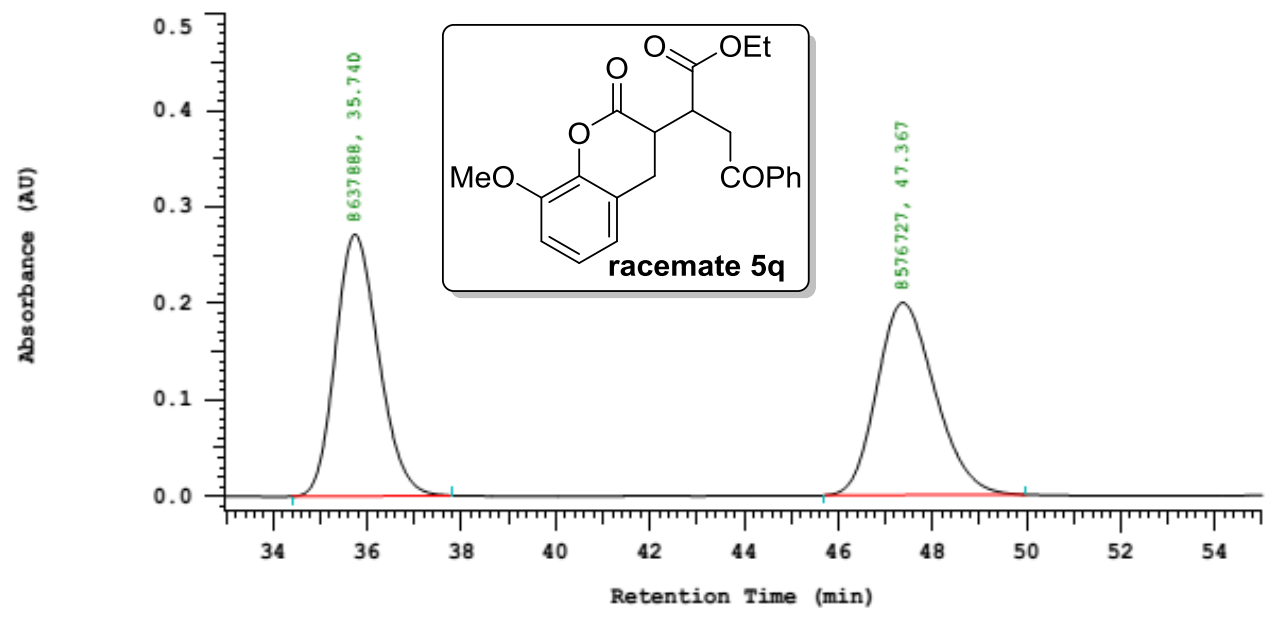

Chrom Type: Fixed WL Chromatogram, $210 \mathrm{~nm}$

Peak Quantitation: AREA

Calculation Method: AREA

\begin{tabular}{ccccc} 
No. & RT & Area & Area \& & BC \\
\hline 1 & 35.740 & 8637888 & 50.178 & BB \\
2 & 47.367 & 8576727 & 49.822 & BB \\
\hline & 17214615 & 100.000 & \\
\hline
\end{tabular}

Chrom Type: Fixed WL Chromatogram, $210 \mathrm{~nm}$

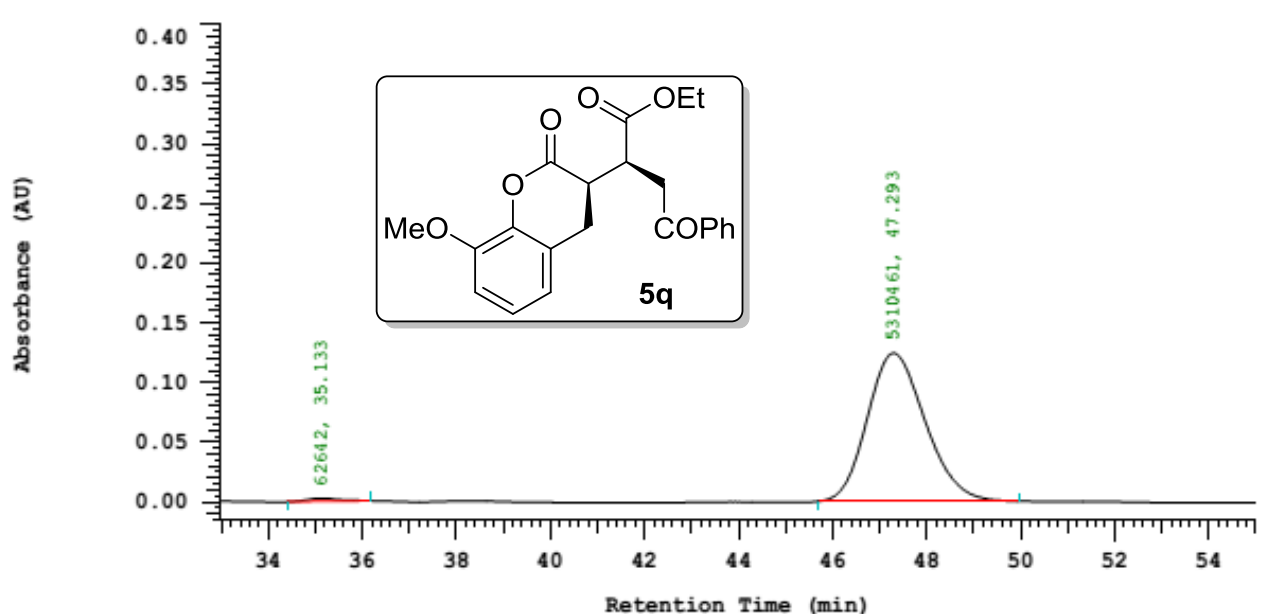

Chrom Type: Fixed WL Chromatogram, $210 \mathrm{~nm}$

Peak Quantitation: AREA

Calculation Method: AREA용

\begin{tabular}{ccccc} 
No. & RT & Area & Area o & BC \\
\hline 1 & 35.133 & 62642 & 1.166 & BB \\
2 & 47.293 & 5310461 & 98.834 & BB \\
\hline & & 5373103 & 100.000 & \\
\hline
\end{tabular}



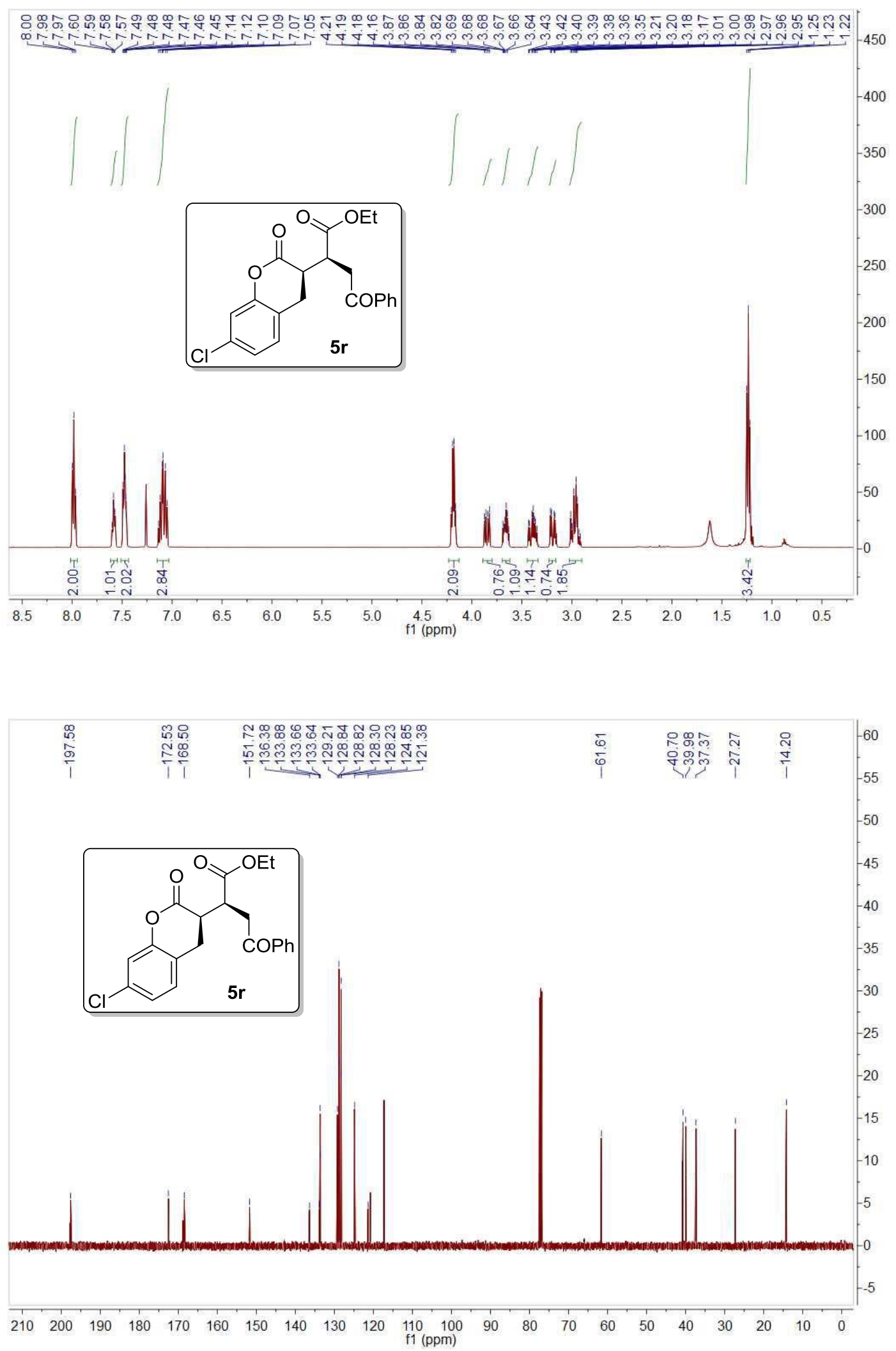
Chrom Type: Fixed WL Chromatogram, $210 \mathrm{~nm}$

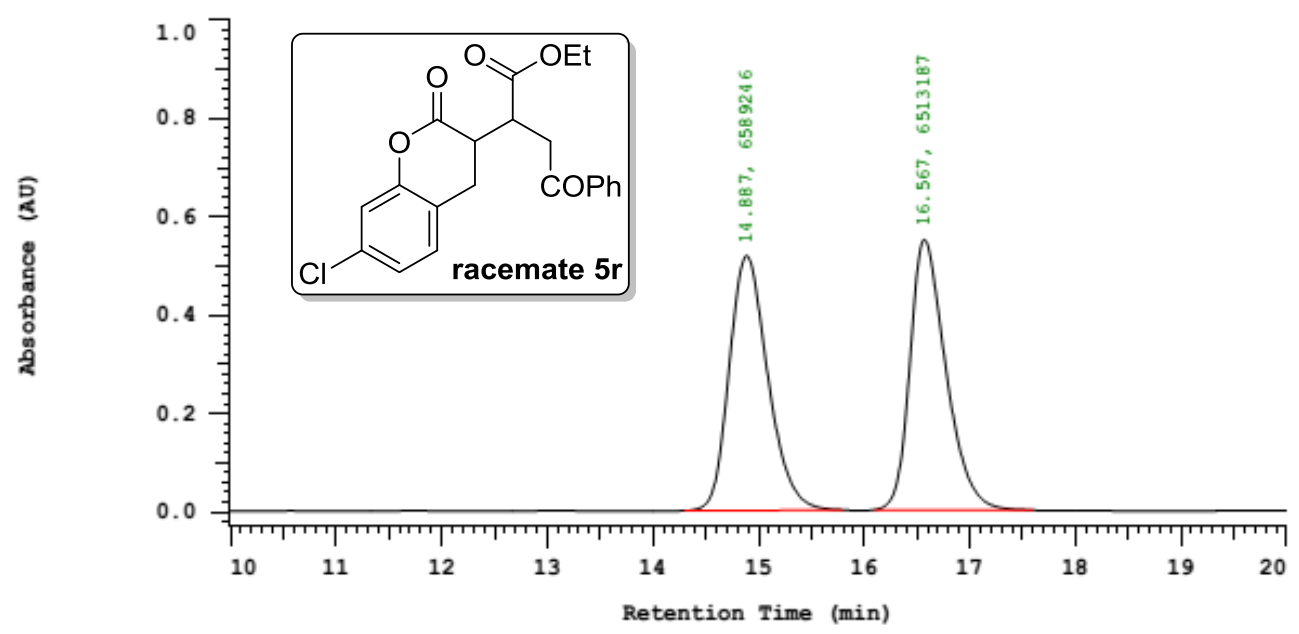

Chrom Type: Fixed WL Chromatogram, $210 \mathrm{~nm}$

Peak Quantitation: AREA

Calculation Method: AREA음

\begin{tabular}{ccccc} 
No. & RT & Area & Area \& & BC \\
\hline 1 & 14.887 & 6589246 & 50.290 & BB \\
2 & 16.567 & 6513187 & 49.710 & BB \\
\hline & & 13102433 & 100.000 & \\
\hline
\end{tabular}

Chrom Type: Fixed WL Chromatogram, $210 \mathrm{~nm}$

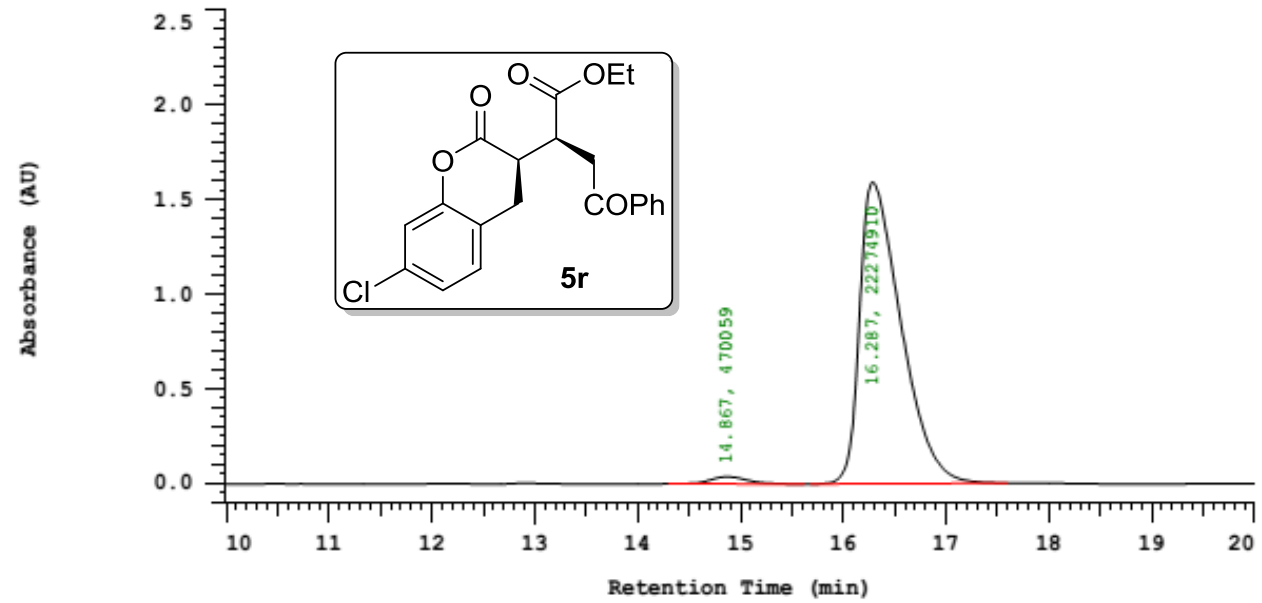

Chrom Type: Fixed WL Chromatogram, $210 \mathrm{~nm}$

Peak Quantitation: AREA

Calculation Method: AREA용

\begin{tabular}{rrrrr} 
No. & RT & \multicolumn{1}{c}{ Area } & Area 8 & BC \\
\hline 1 & 14.867 & 470059 & 2.067 & BB \\
2 & 16.287 & 22274910 & 97.933 & BB \\
\hline & & 22744969 & 100.000 & \\
\hline
\end{tabular}



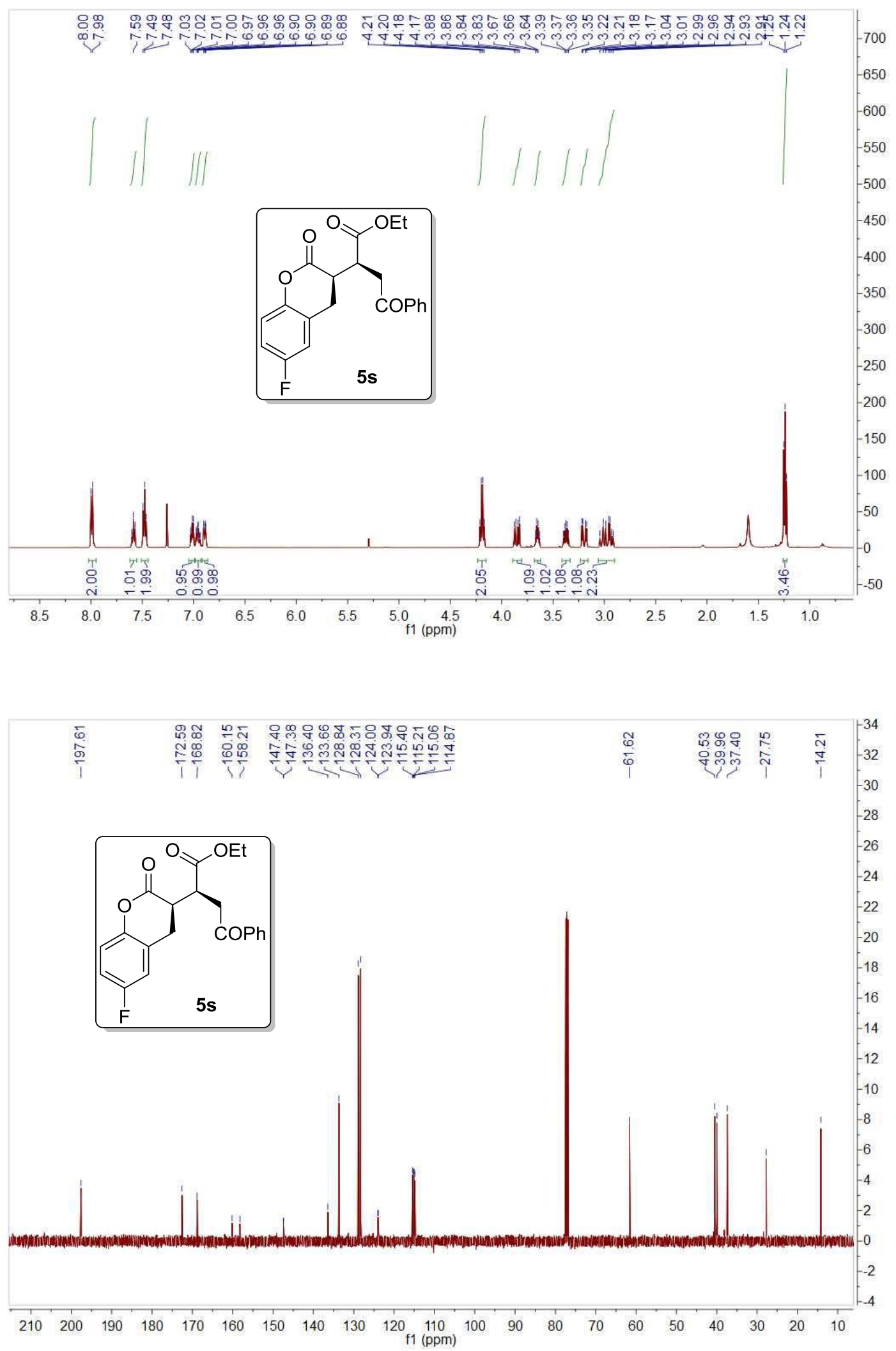
Chrom Type: Fixed WL Chromatogram, $210 \mathrm{~nm}$

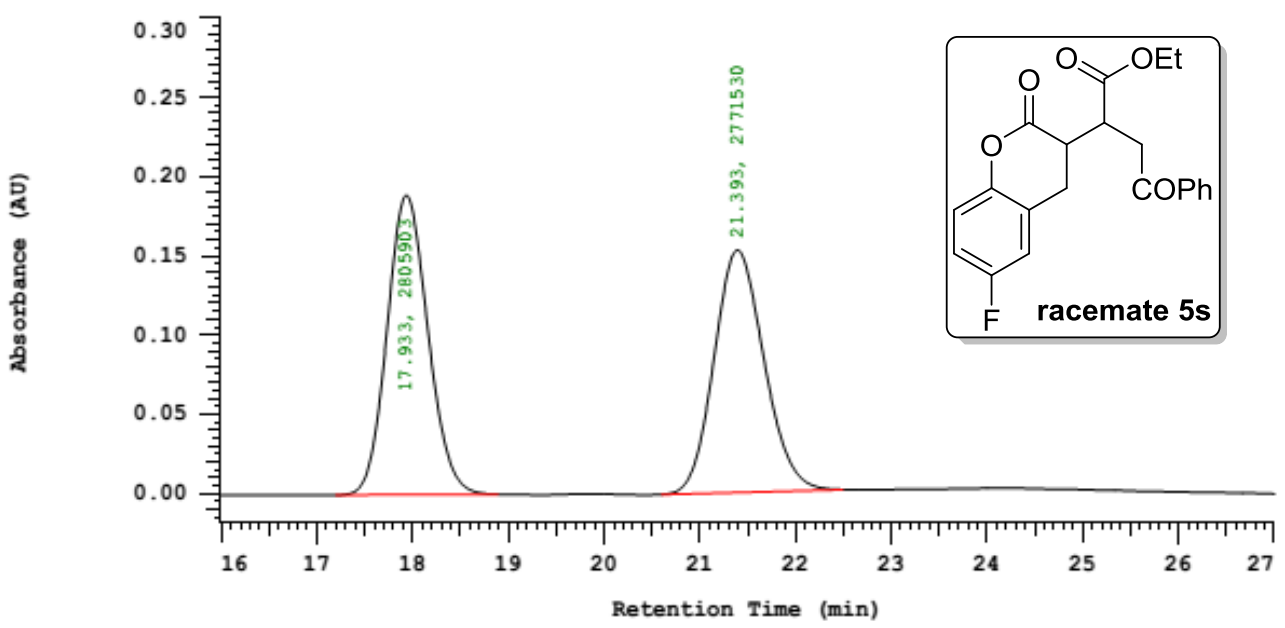

Chrom Type: Fixed WL Chromatogram, $210 \mathrm{~nm}$

Peak Quantitation: AREA

Calculation Method: AREA용

\begin{tabular}{ccccc} 
No. & RT & Area & Area o & BC \\
\hline 1 & 17.933 & 2805903 & 50.308 & BB \\
2 & 21.393 & 2771530 & 49.692 & BB \\
\hline & & 5577433 & 100.000 & \\
\hline
\end{tabular}

Chrom Type: Fixed WL Chromatogram, $210 \mathrm{~nm}$

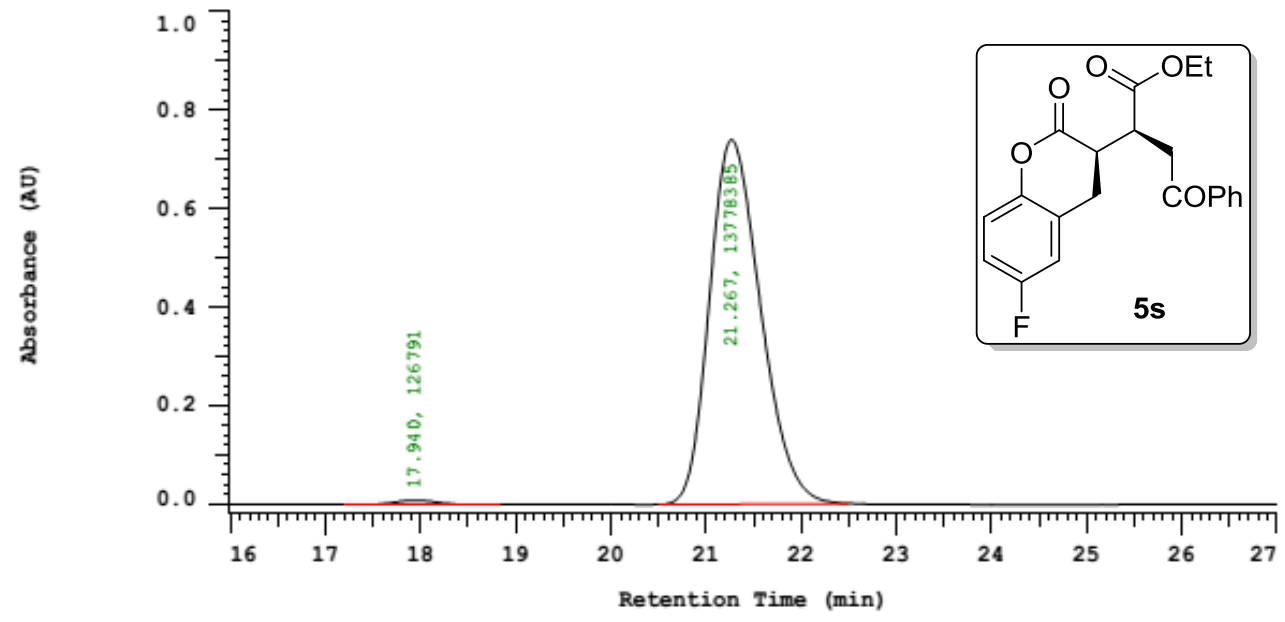

Chrom Type: Fixed WL Chromatogram, $210 \mathrm{~nm}$

Peak Quantitation: AREA

Calculation Method: AREA

\begin{tabular}{crrrr} 
No. & RT & \multicolumn{1}{c}{ Area } & Area \& & BC \\
\hline 1 & 17.940 & 126791 & 0.912 & BB \\
2 & 21.267 & 13778385 & 99.088 & BB \\
\hline & & 13905176 & 100.000 & \\
\hline
\end{tabular}



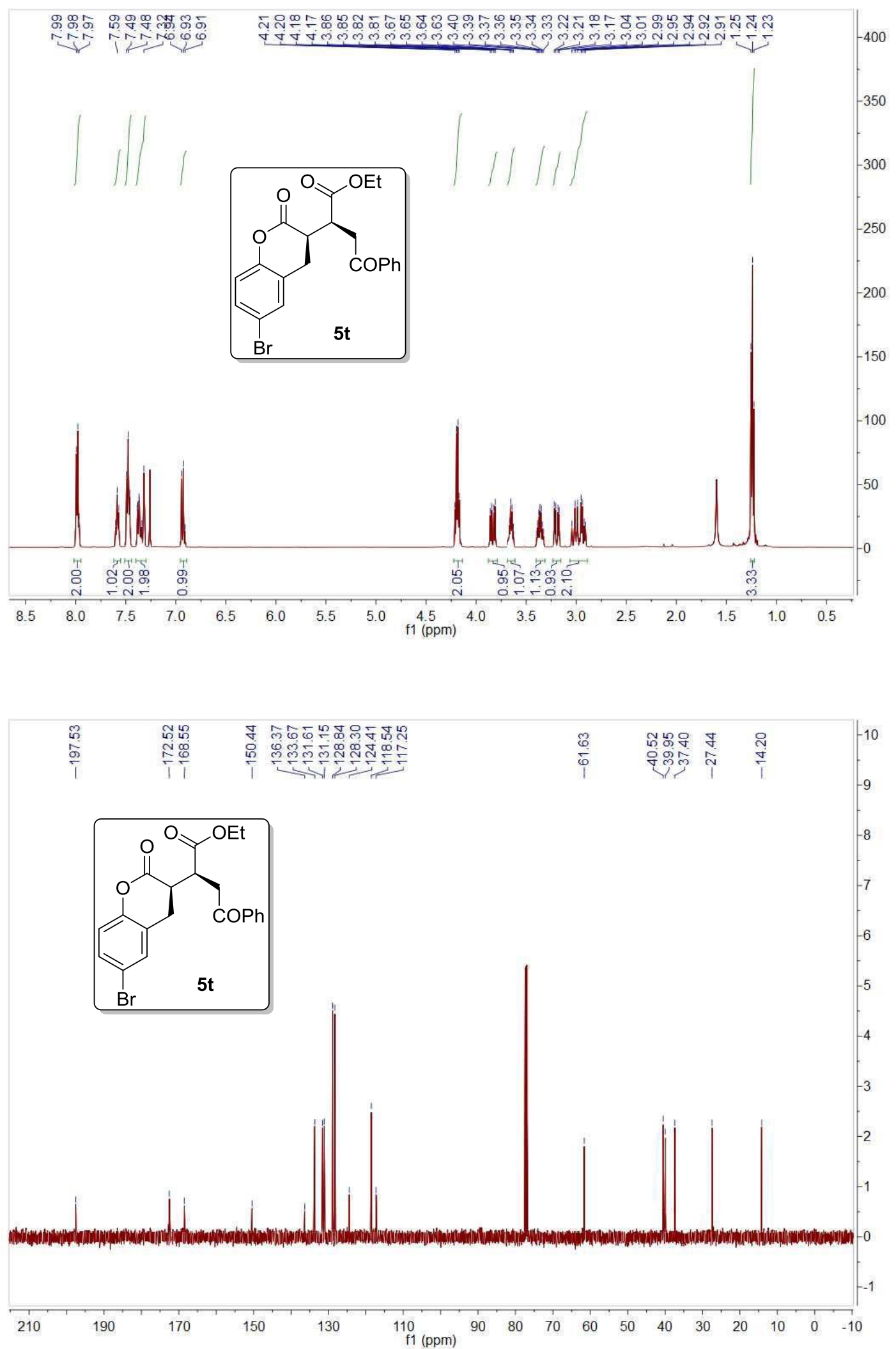
Chrom Type: Fixed WL Chromatogram, 210 nm

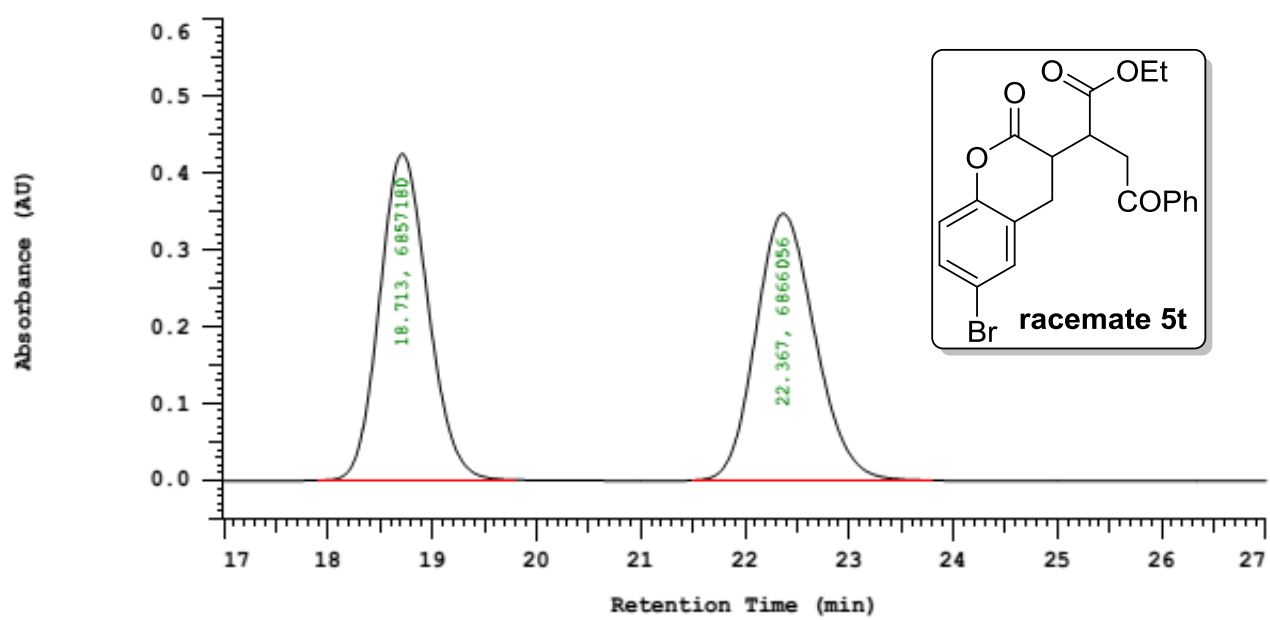

Chrom Type: Fixed WL Chromatogram, $210 \mathrm{~nm}$

Peak Quantitation: AREA

Calculation Method: AREA\&

\begin{tabular}{ccccc} 
No. & RT & Area & Area 8 & BC \\
\hline 1 & 18.713 & 6857180 & 49.968 & BB \\
2 & 22.367 & 6866056 & 50.032 & BB \\
\hline & & 13723236 & 100.000 & \\
\hline
\end{tabular}

Chrom Type: Fixed WL Chromatogram, $210 \mathrm{~nm}$

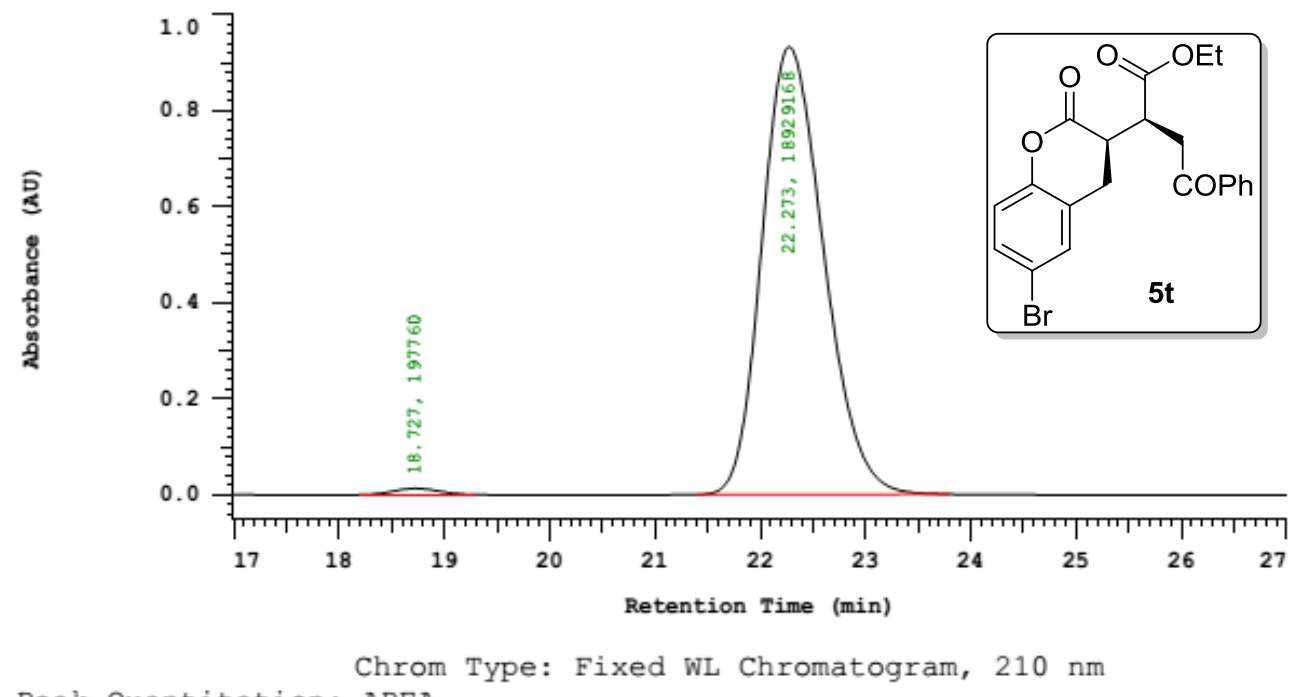

Peak Quantitation: AREA

Calculation Method: AREA

\begin{tabular}{ccrrr} 
No. & RT & \multicolumn{1}{c}{ Area } & Area \& & BC \\
\hline 1 & 18.727 & 197760 & 1.034 & BB \\
2 & 22.273 & 18929168 & 98.966 & BB \\
\hline & 19126928 & 100.000 & \\
\hline
\end{tabular}



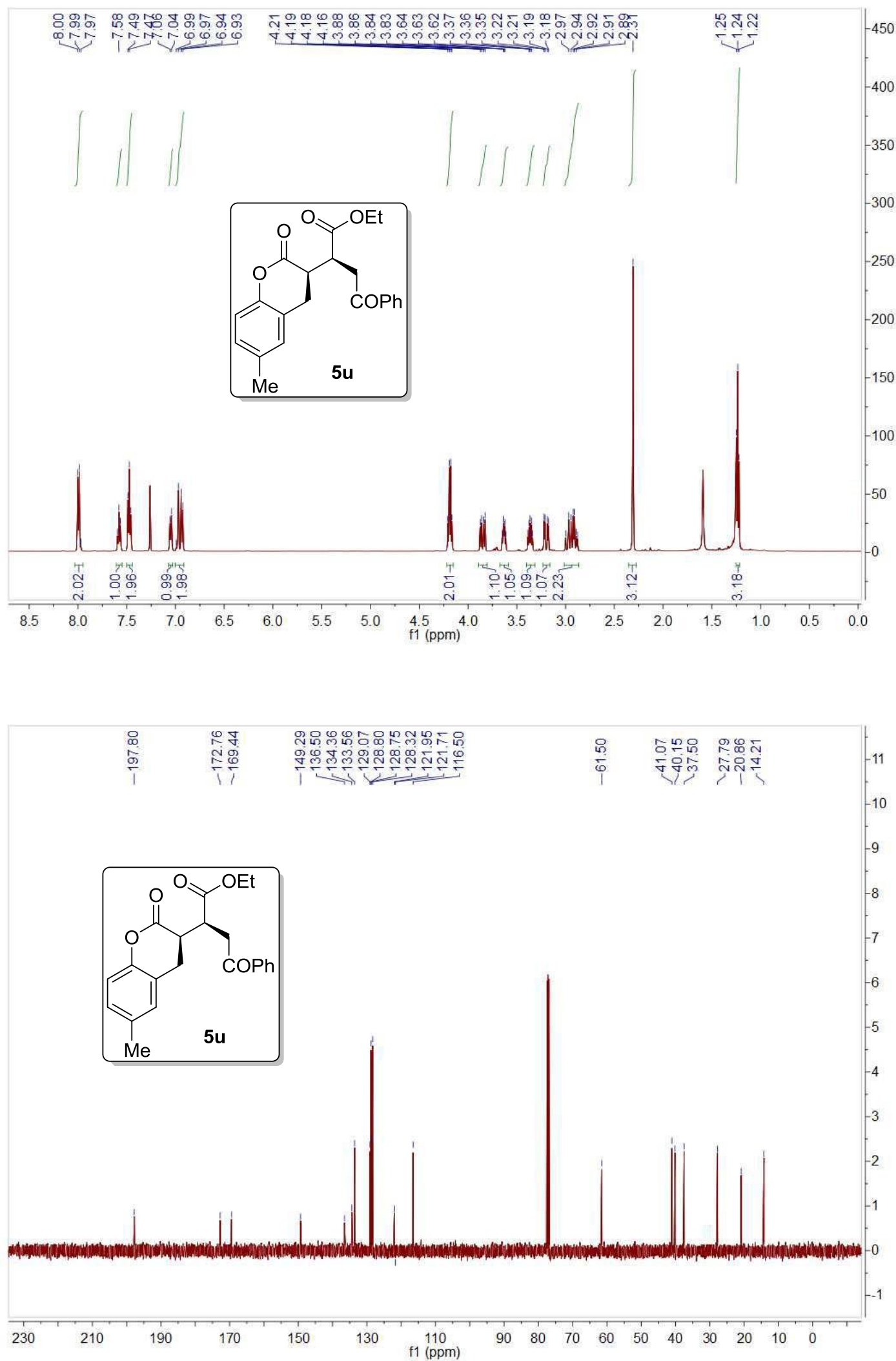
Chrom Type: Fixed WL Chromatogram, $210 \mathrm{~nm}$

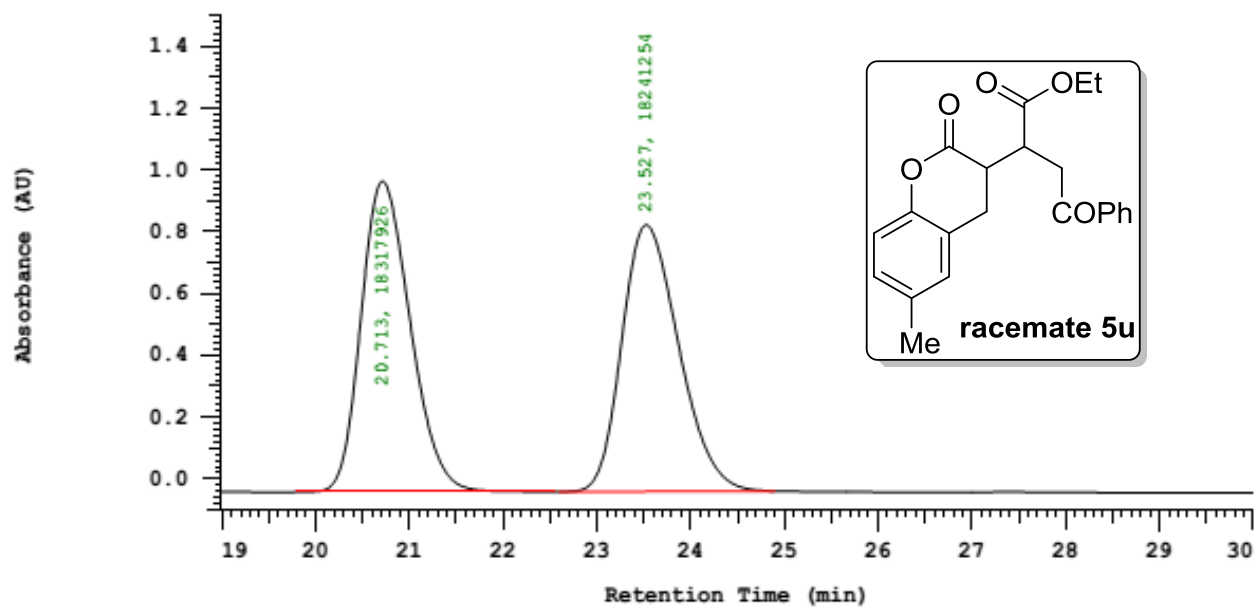

Chrom Type: Fixed WL Chromatogram, $210 \mathrm{~nm}$

Peak Quantitation: AREA

Calculation Method: AREA\&

\begin{tabular}{ccccc} 
No. & RT & Area & Area o & BC \\
\hline 1 & 20.713 & 18317926 & 50.105 & BB \\
2 & 23.527 & 18241254 & 49.895 & BB \\
\hline & & 36559180 & 100.000 & \\
\hline
\end{tabular}

Chrom Type: Fixed WL Chromatogram, $210 \mathrm{~nm}$

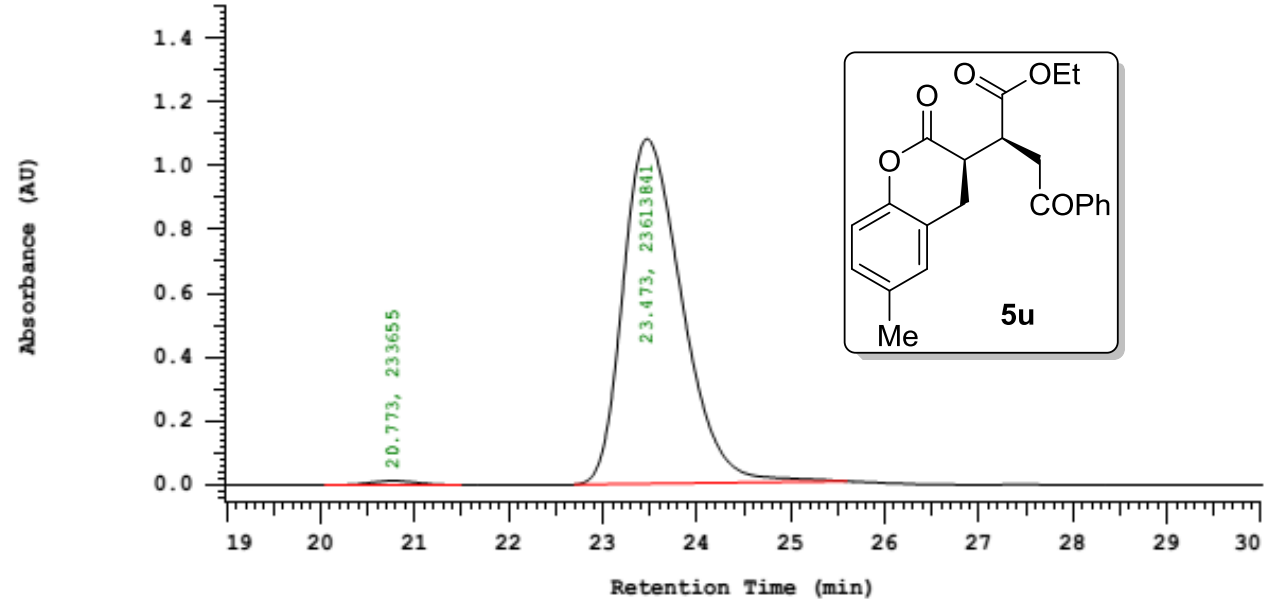

Chrom Type: Fixed WL Chromatogram, $210 \mathrm{~nm}$

Peak Quantitation: AREA

Calculation Method: AREA8

\begin{tabular}{ccccc} 
No. & RT & \multicolumn{1}{c}{ Area } & Area 8 & BC \\
\hline 1 & 20.773 & 233655 & 0.980 & BB \\
2 & 23.473 & 23613841 & 99.020 & BB \\
\hline & & 23847496 & 100.000
\end{tabular}



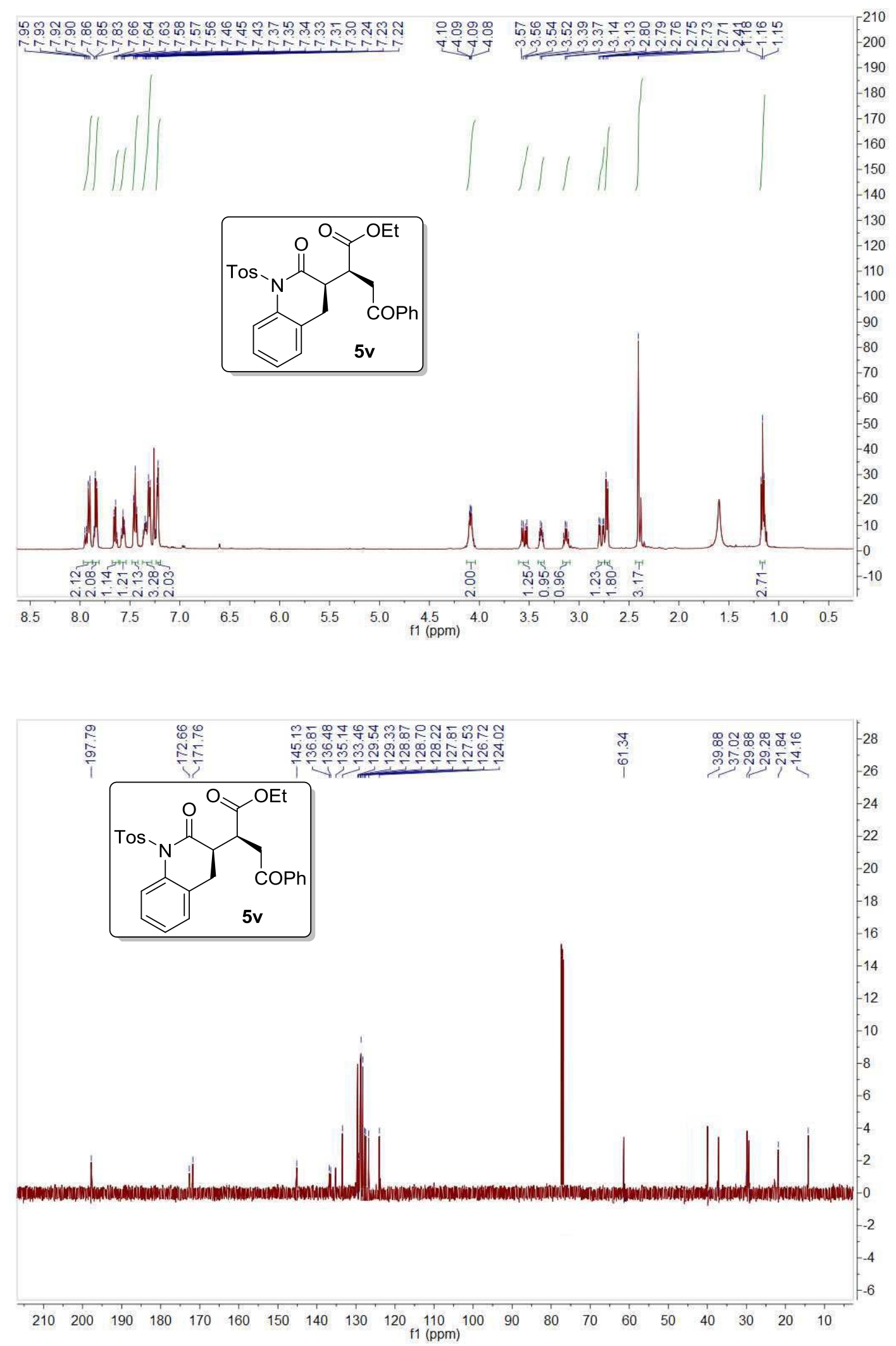
Chrom Type: Fixed WL Chromatogram, $210 \mathrm{~nm}$

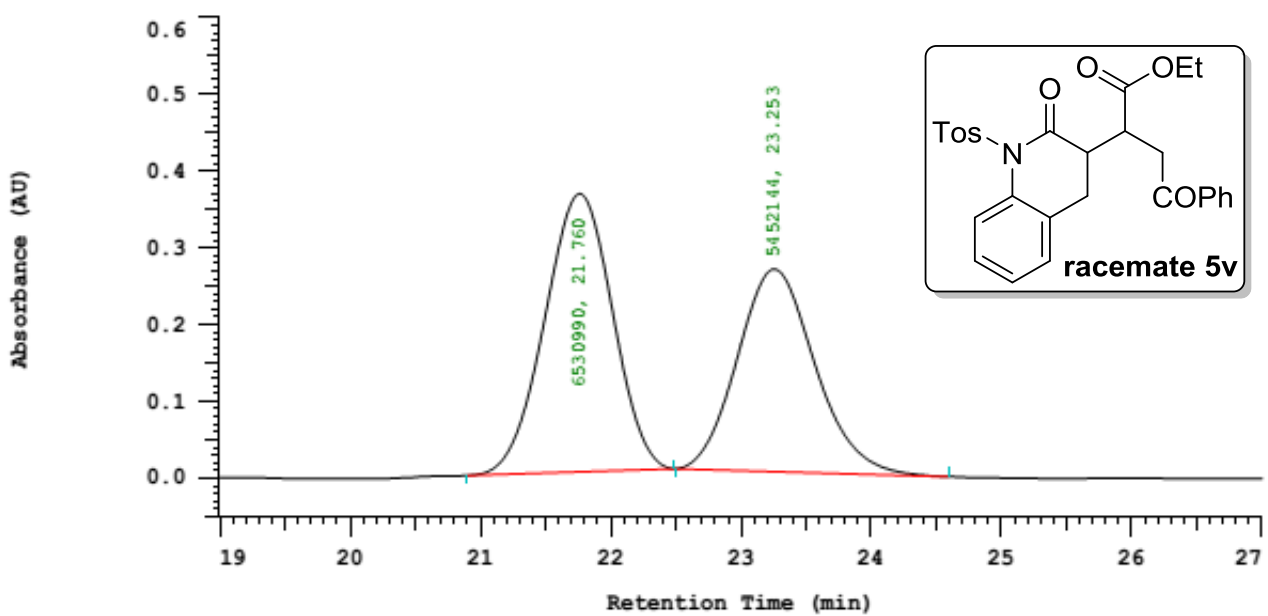

Chrom Type: Fixed WL Chromatogram, $210 \mathrm{~nm}$

Peak Quantitation: AREA

Calculation Method: AREAs

\begin{tabular}{ccccc} 
No. & RT & Area & Area 8 & BC \\
\hline 1 & 21.760 & 6530990 & 54.502 & BB \\
2 & 23.253 & 5452144 & 45.498 & BB \\
\hline & & 11983134 & 100.000
\end{tabular}

Chrom Type: Fixed WL Chromatogram, $210 \mathrm{~nm}$

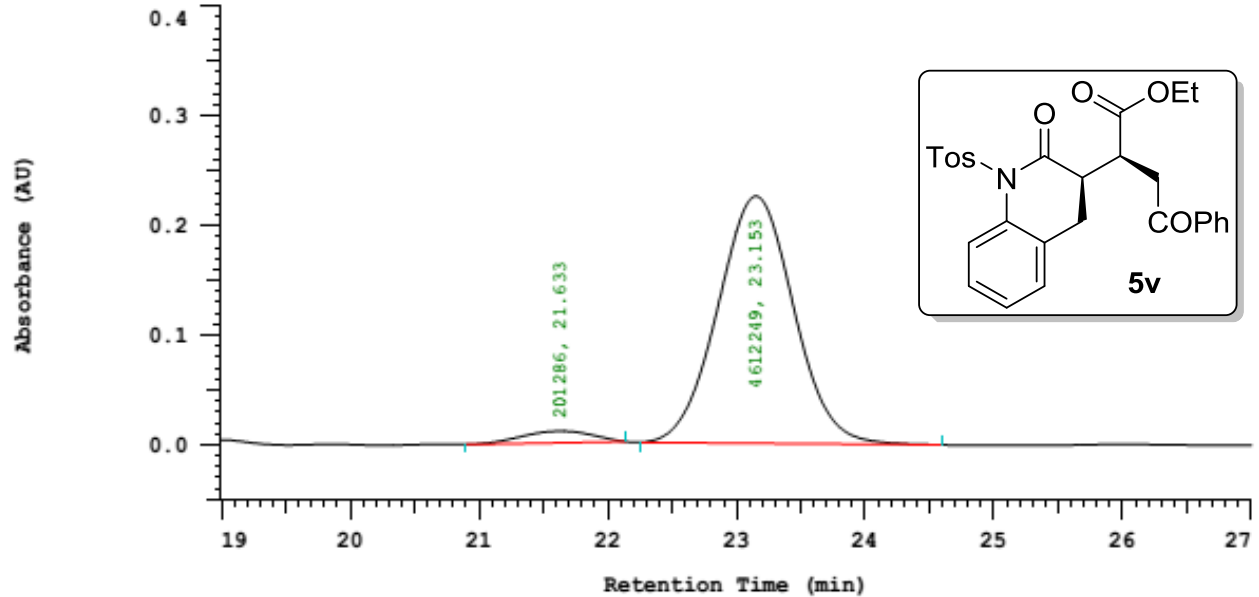

Chrom Type: Fixed WL Chromatogram, $210 \mathrm{~nm}$

Peak Quantitation: AREA

Calculation Method: AREA용

\begin{tabular}{rrrrr} 
No. & RT & Area & Area o & BC \\
\hline 1 & 21.633 & 201286 & 4.182 & BB \\
2 & 23.153 & 4612249 & 95.818 & BB \\
\hline & & 4813535 & 100.000
\end{tabular}



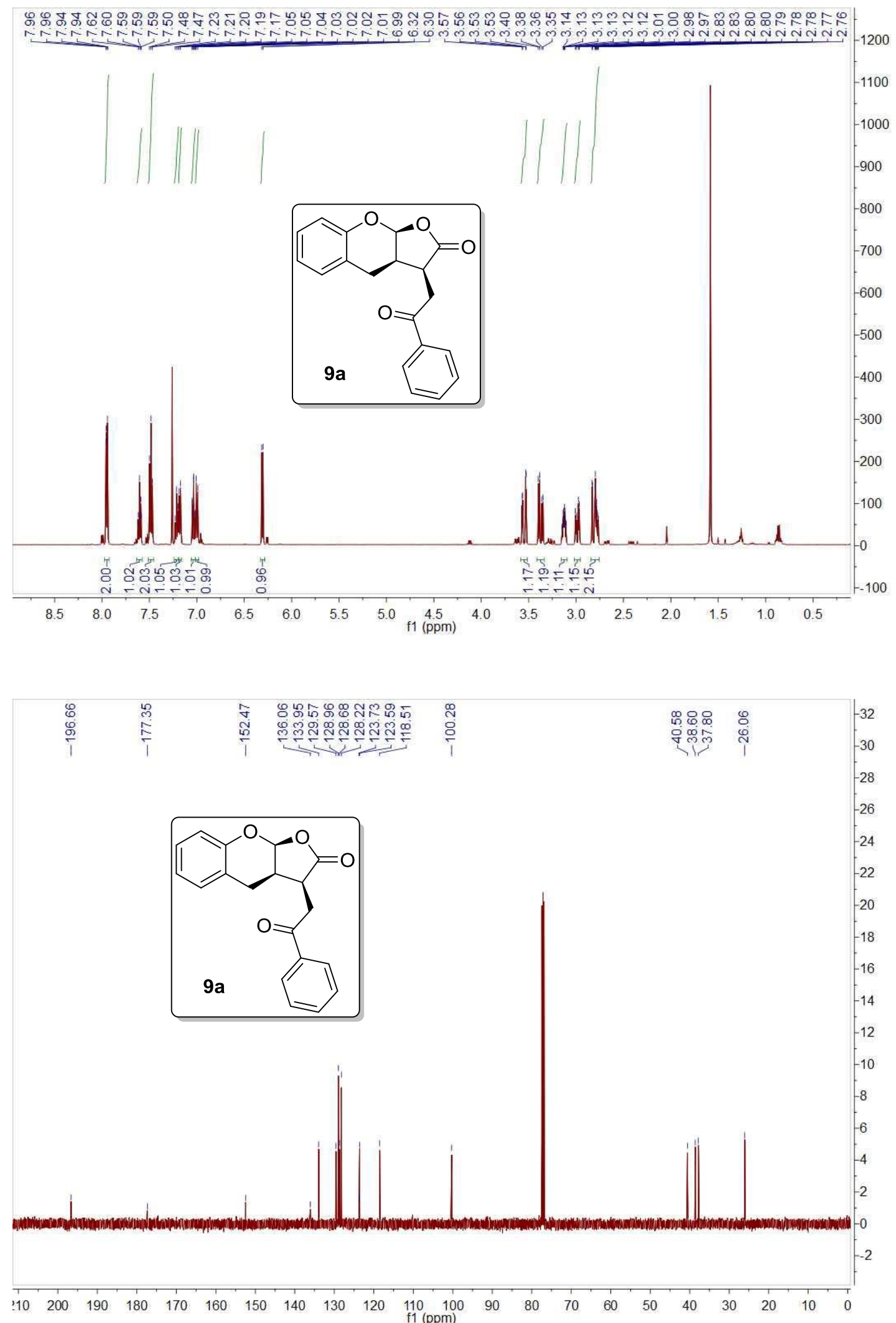
Chrom Type: Fixed WL Chromatogram, $210 \mathrm{~nm}$

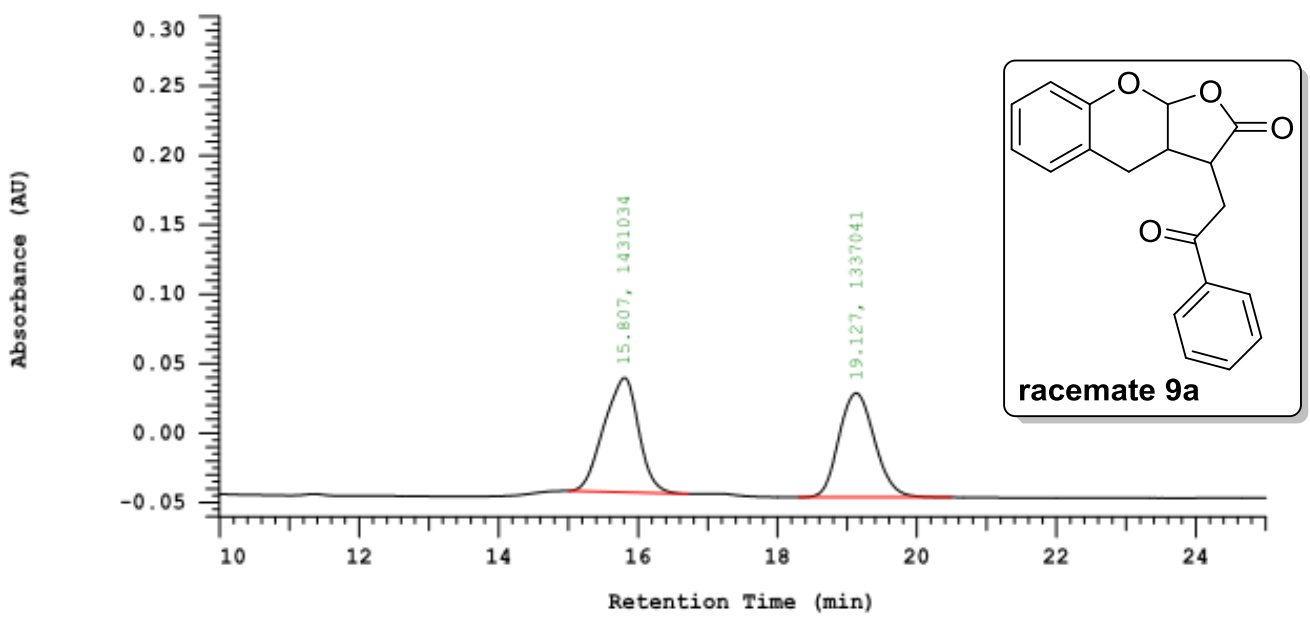

Chrom Type: Fixed WL Chromatogram, $210 \mathrm{~nm}$

Peak Quantitation: AREA

Calculation Method: AREA응

\begin{tabular}{ccccc} 
No. & RT & Area & Area \% & BC \\
\hline 1 & 15.807 & 1431034 & 51.698 & BB \\
2 & 19.127 & 1337041 & 48.302 & BB \\
\hline & & 2768075 & 100.000 & \\
\hline
\end{tabular}

Chrom Type: Fixed WL Chromatogram, $210 \mathrm{~nm}$

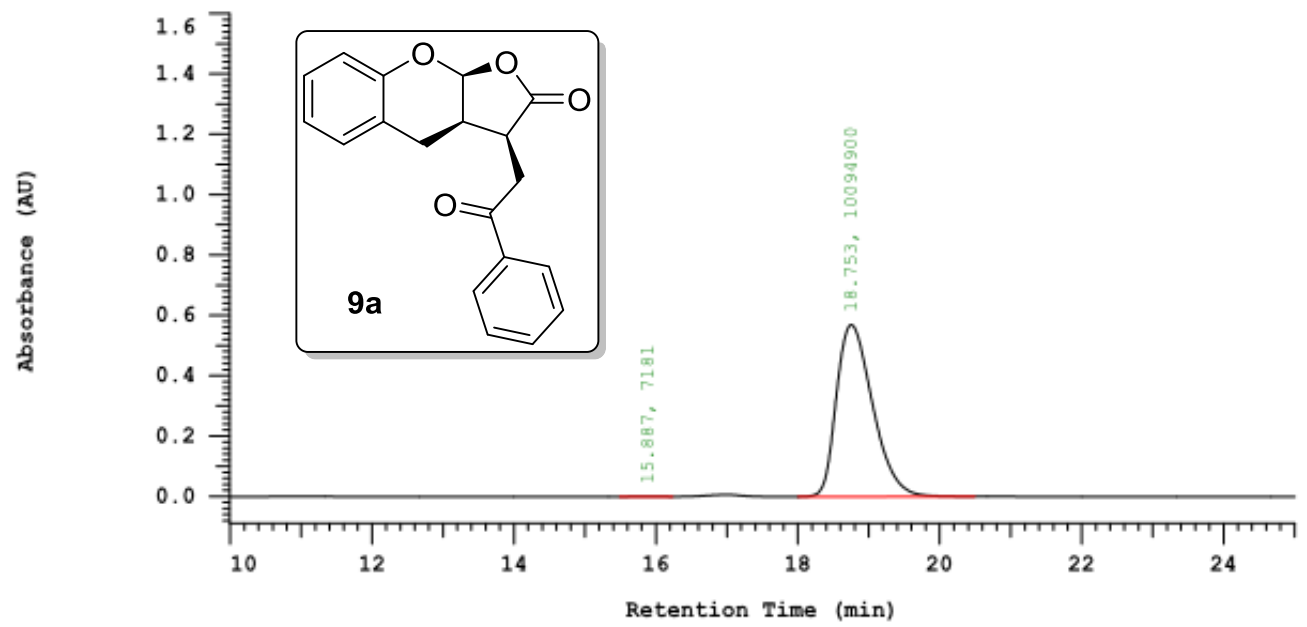

Chrom Type: Fixed WL Chromatogram, $210 \mathrm{~nm}$

Peak Quantitation: AREA

Calculation Method: AREA\%

\begin{tabular}{|c|c|c|c|c|}
\hline o. & RT & Area & Area \% & $\mathrm{BC}$ \\
\hline 1 & 15.887 & 7181 & 0.071 & $\mathrm{BB}$ \\
\hline 2 & 18.753 & 10094900 & 99.929 & BB \\
\hline & & 10102081 & 100.000 & \\
\hline
\end{tabular}



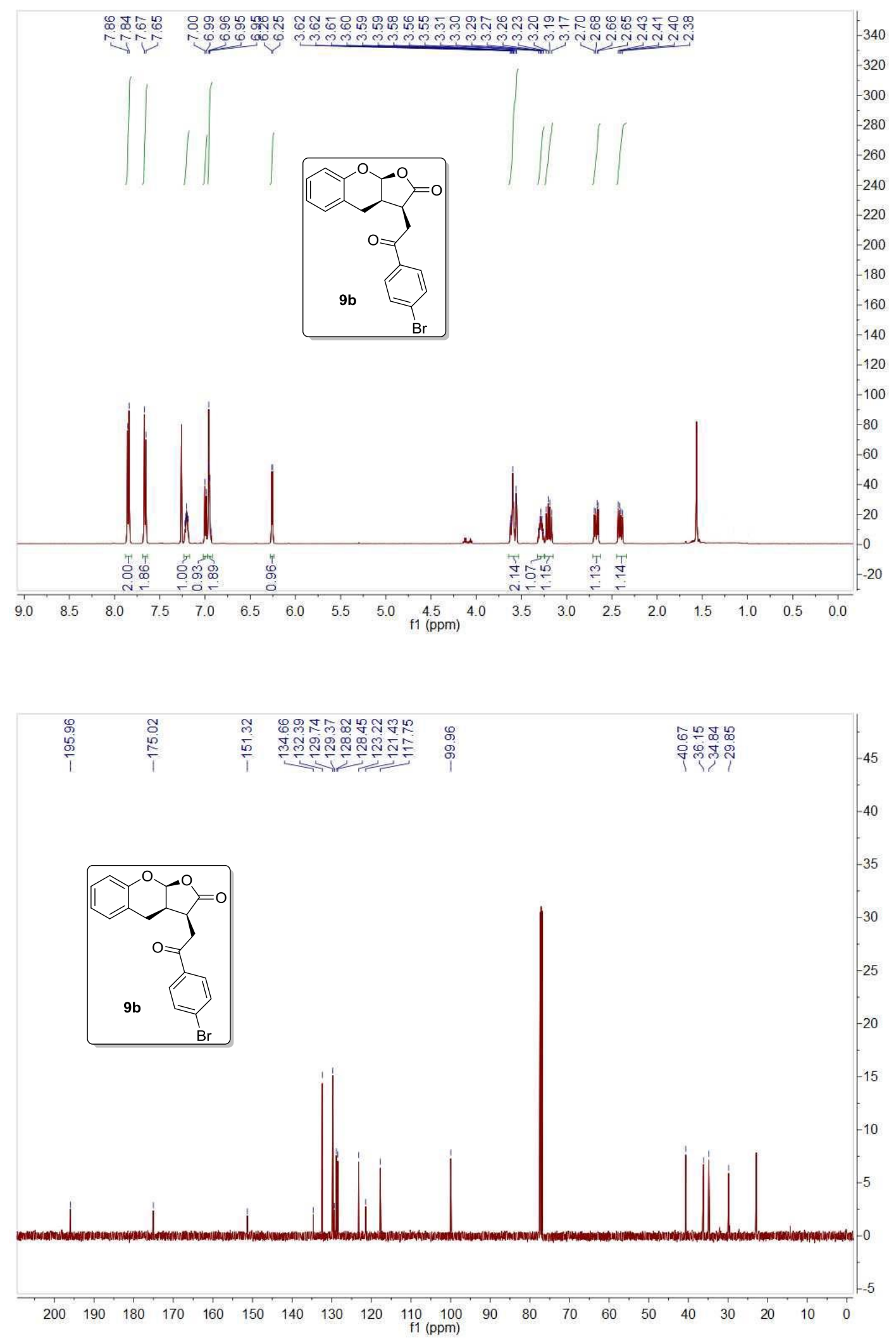


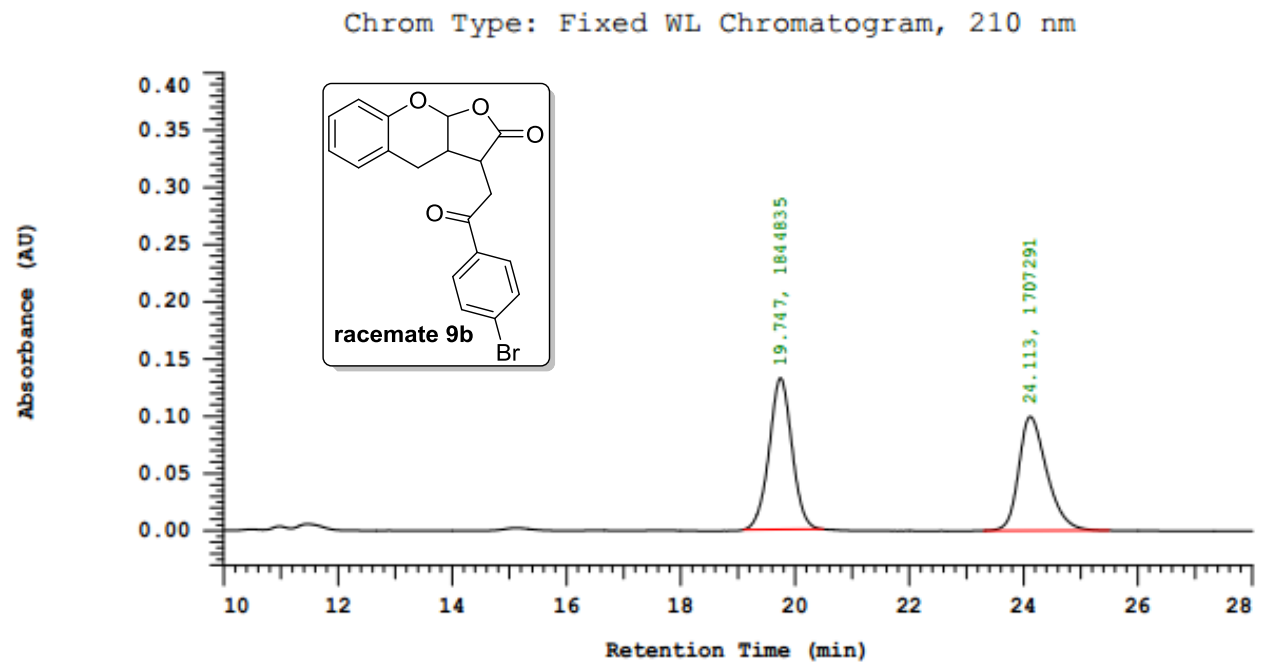

Peak Quantitation: AREA

Calculation Method: AREA

\begin{tabular}{ccccc} 
No. & RT & Area & Area 8 & BC \\
\hline 1 & 19.747 & 1844835 & 51.936 & BB \\
2 & 24.113 & 1707291 & 48.064 & BB \\
\hline & & 3552126 & 100.000 & \\
\hline
\end{tabular}

Chrom Type: Fixed WL Chromatogram, 210 nm

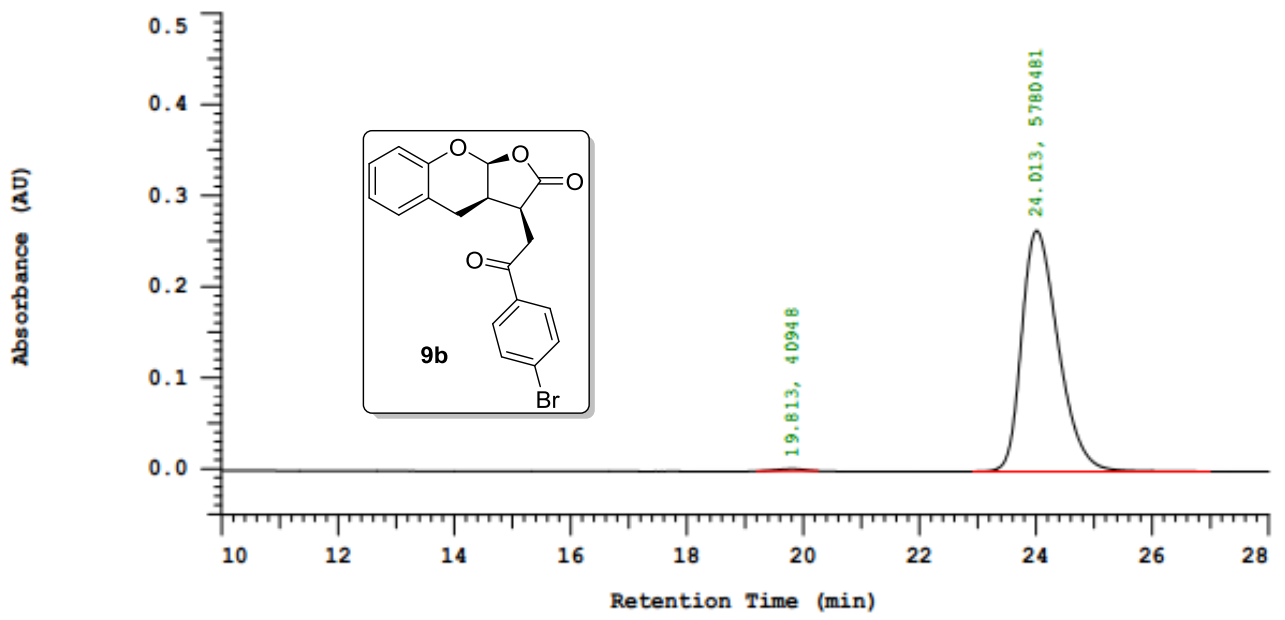

Chrom Type: Fixed WL Chromatogram, $210 \mathrm{~nm}$

Peak Quantitation: AREA

Calculation Method: AREA

\begin{tabular}{ccrrr} 
No. & RT & Area & Area 8 & BC \\
\hline 1 & 19.813 & 40948 & 0.703 & BB \\
2 & 24.013 & 5780481 & 99.297 & BB \\
\hline & & 5821429 & 100.000 &
\end{tabular}



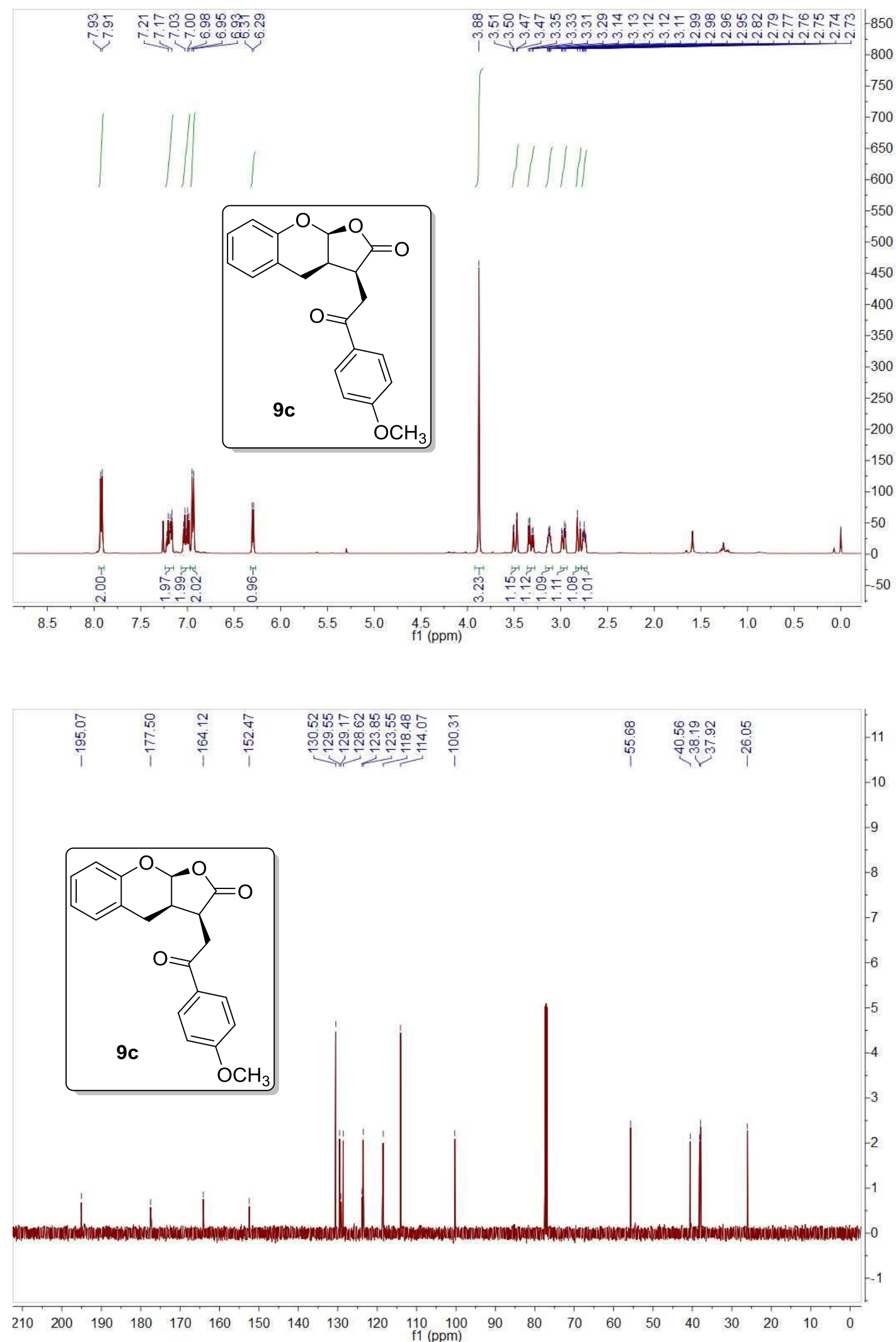
Chrom Type: Fixed WL Chromatogram, $210 \mathrm{~nm}$

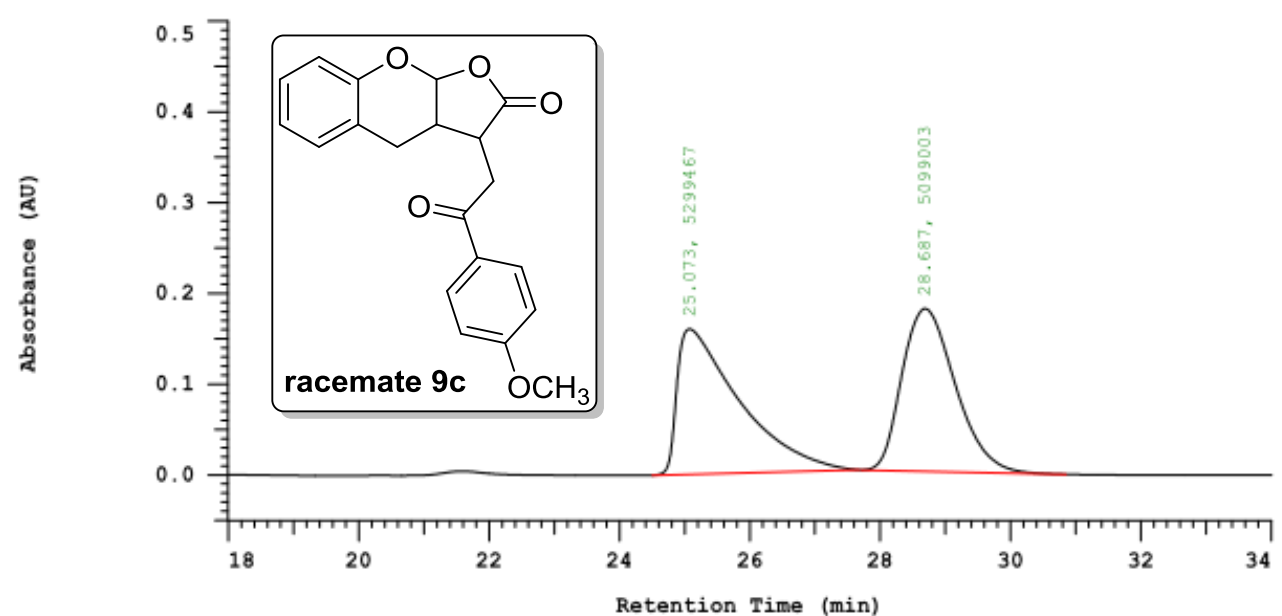

Chrom Type: Fixed WL Chromatogram, $210 \mathrm{~nm}$

Peak Quantitation: AREA

Calculation Method: AREA응

\begin{tabular}{ccccc} 
No. & RT & Area & Area 음 & BC \\
\hline 1 & 25.073 & 5299467 & 50.964 & BB \\
2 & 28.687 & 5099003 & 49.036 & BB \\
\hline & 10398470 & 100.000 & \\
\hline
\end{tabular}

Chrom Type: Fixed WL Chromatogram, $210 \mathrm{~nm}$

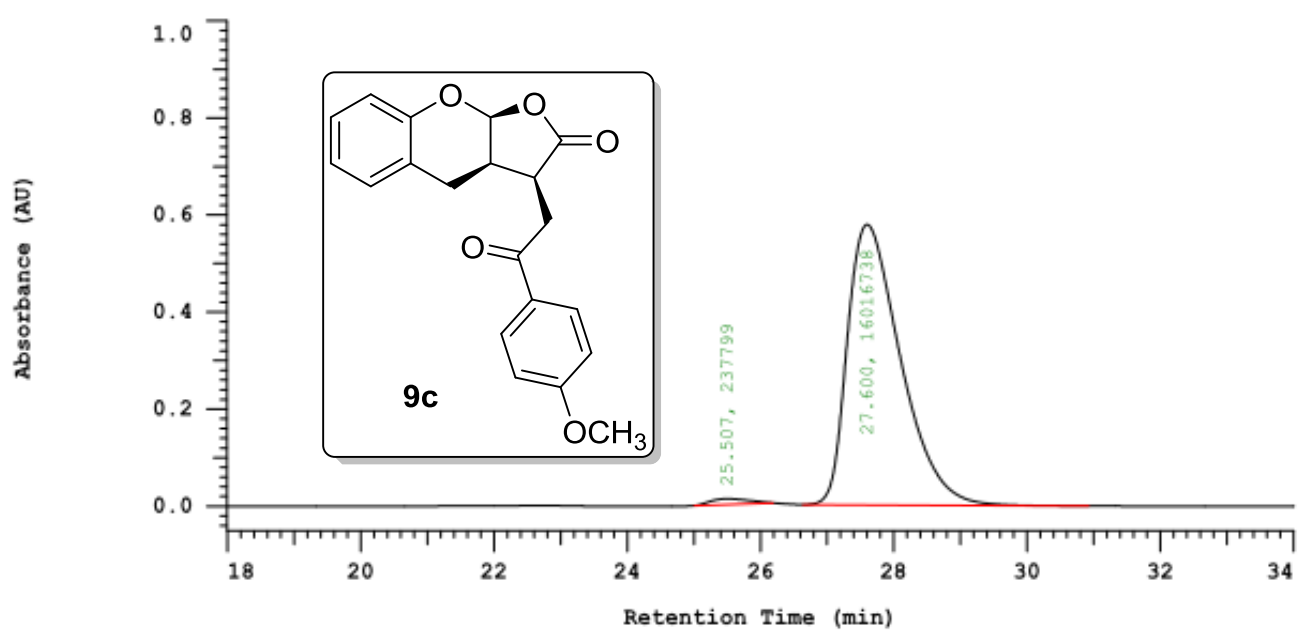

Chrom Type: Fixed WL Chromatogram, $210 \mathrm{~nm}$

Peak Quantitation: AREA

Calculation Method: AREA\%

\begin{tabular}{rrrrr} 
No. & RT & \multicolumn{1}{c}{ Area } & Area \% & BC \\
\hline 1 & 25.507 & 237799 & 1.463 & BB \\
2 & 27.600 & 16016738 & 98.537 & BB \\
\hline & 16254537 & 100.000 & \\
\hline
\end{tabular}



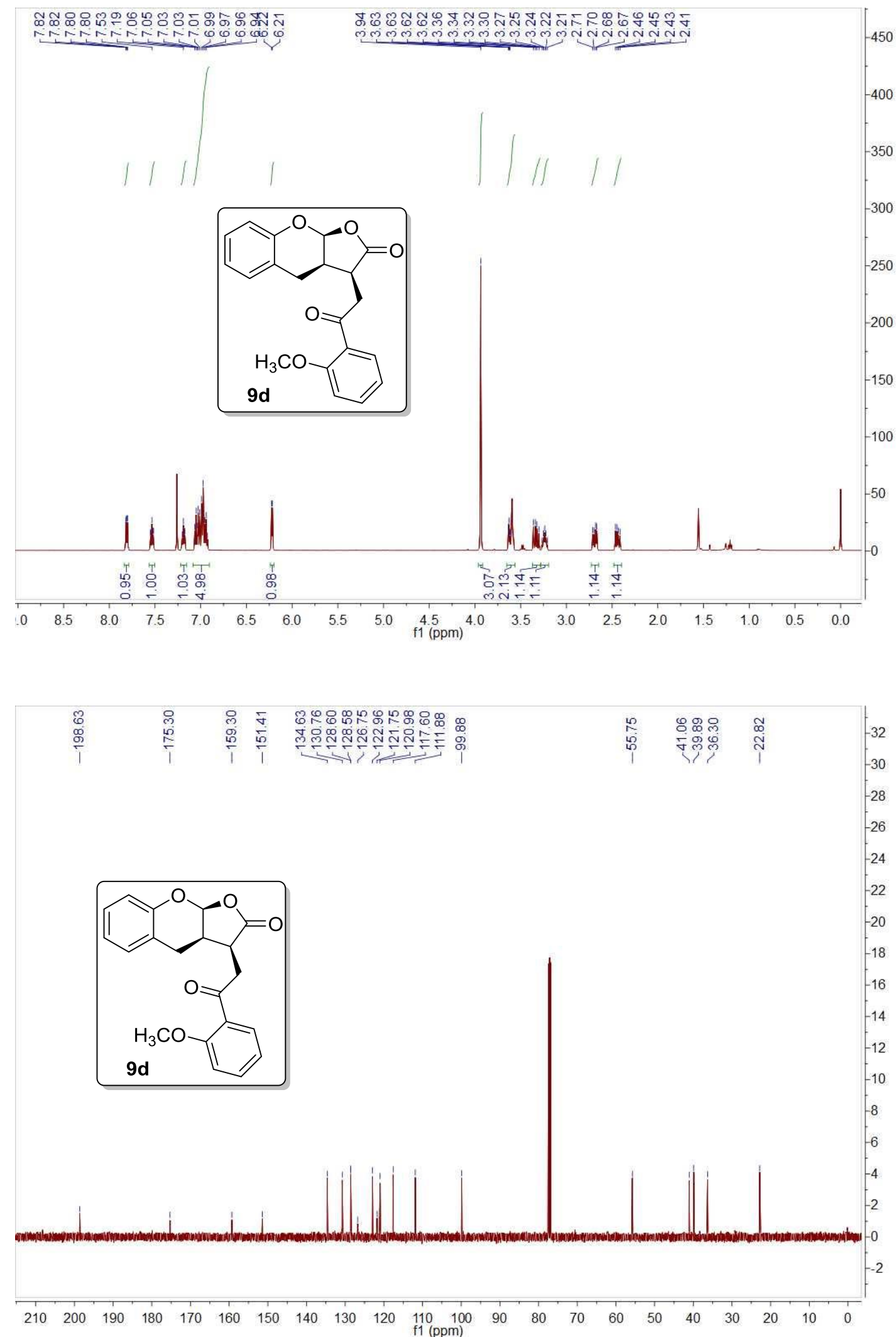


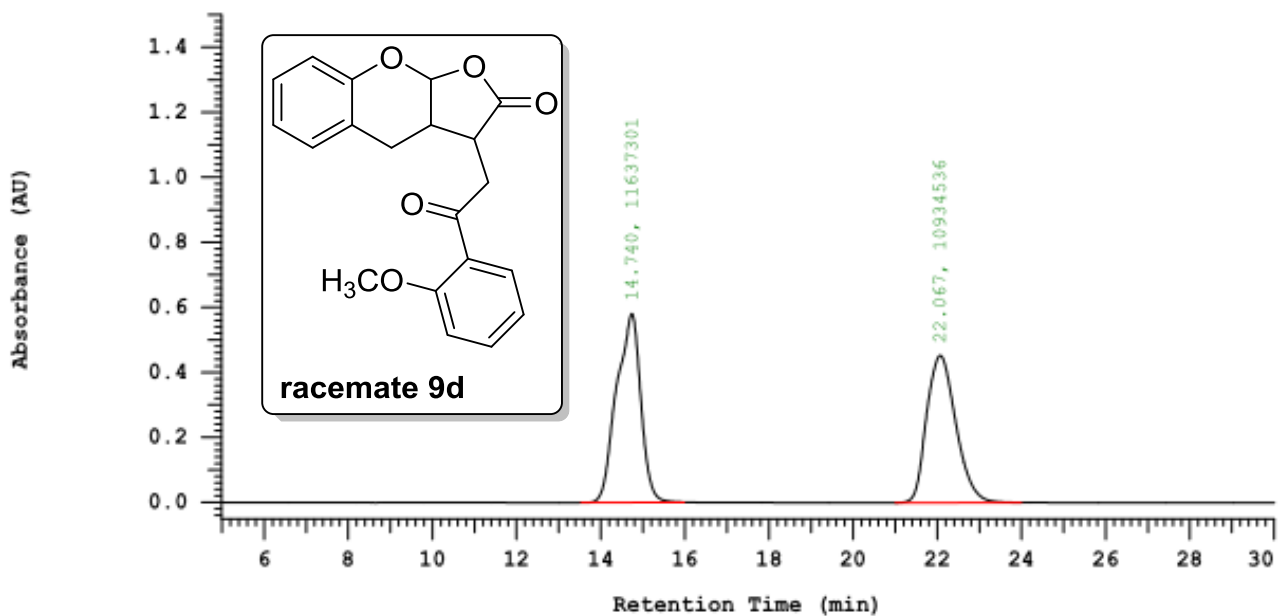

Chrom Type: Fixed WL Chromatogram, $210 \mathrm{~nm}$

Peak Quantitation: AREA

Calculation Method: AREA

\begin{tabular}{ccccc} 
No. & RT & Area & Area & BC \\
\hline 1 & 14.740 & 11637301 & 51.557 & BB \\
2 & 22.067 & 10934536 & 48.443 & BB \\
\hline & 22571837 & 100.000 & \\
\hline
\end{tabular}

Chrom Type: Fixed WL Chromatogram, $210 \mathrm{~nm}$

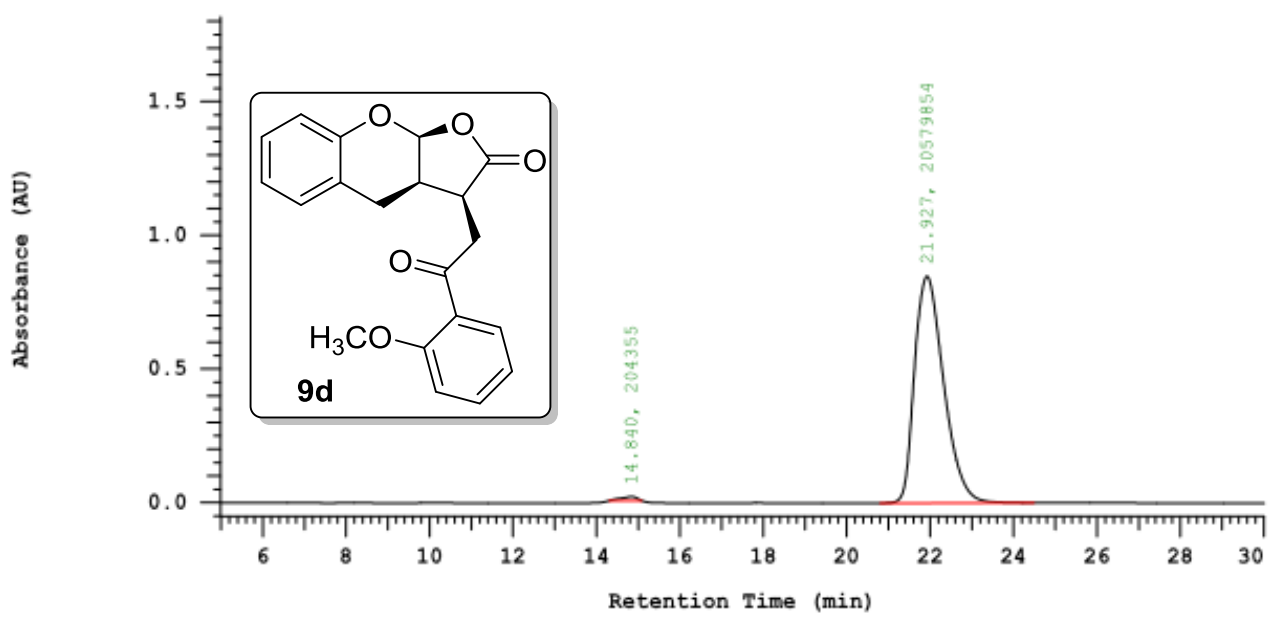

Chrom Type: Fixed WL Chromatogram, $210 \mathrm{~nm}$

Peak Quantitation: AREA

Calculation Method: AREA\%

\begin{tabular}{cccrc} 
No. & RT & \multicolumn{1}{c}{ Area } & Area $\frac{\text { s }}{3}$ & BC \\
\hline 1 & 14.840 & 204355 & 0.983 & BB \\
2 & 21.927 & 20579854 & 99.017 & BB \\
\hline & 20784209 & 100.000 & \\
\hline
\end{tabular}



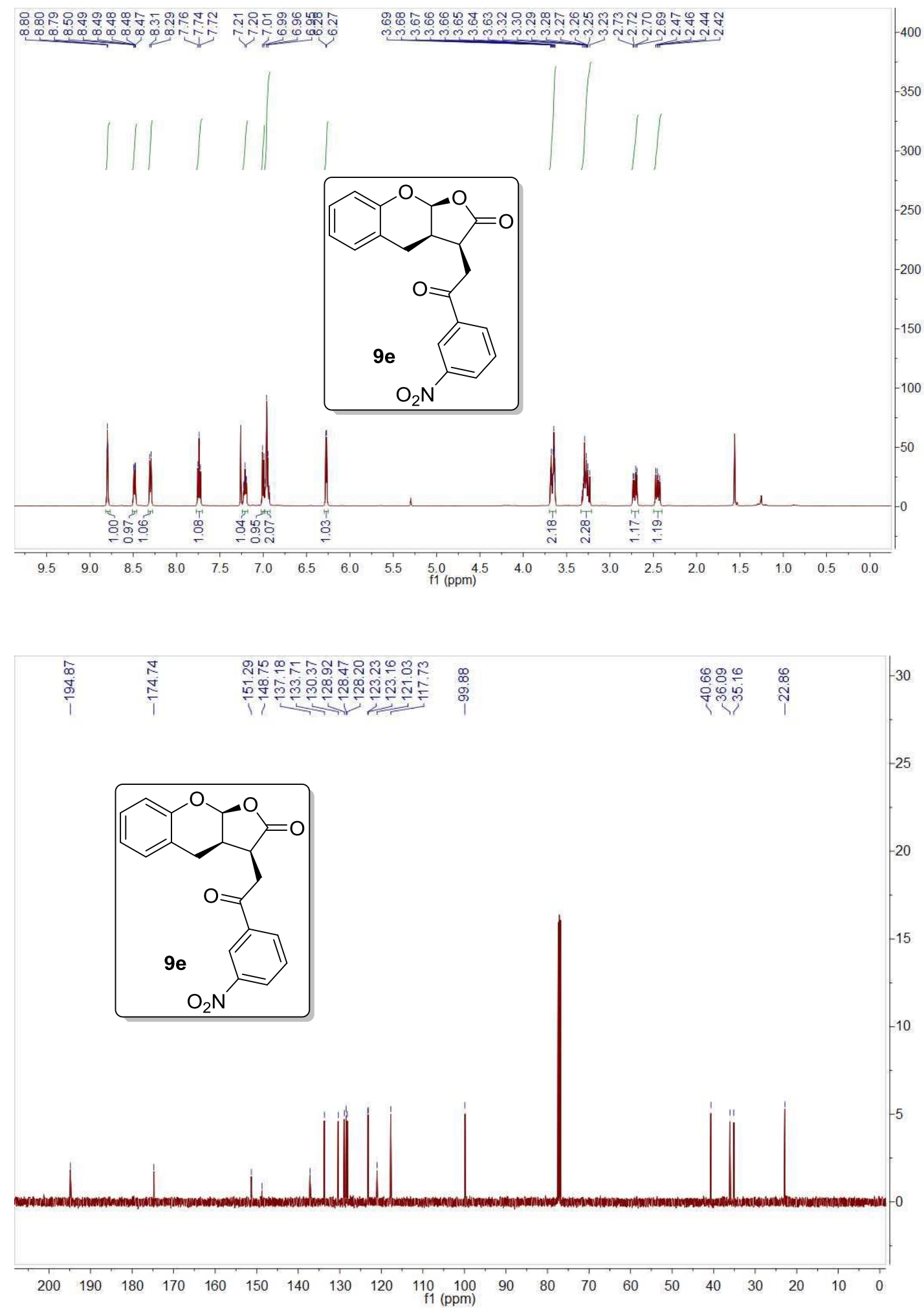
Chrom Type: Fixed WL Chromatogram, $210 \mathrm{~nm}$

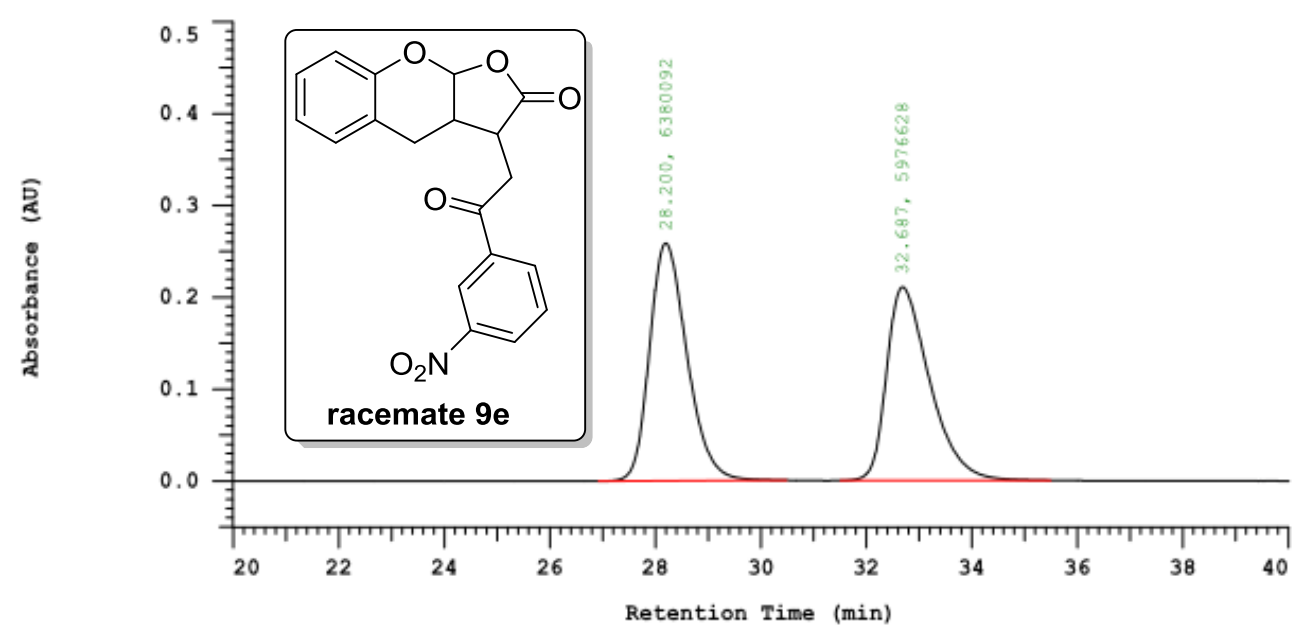

Chrom Type: Fixed WL Chromatogram, $210 \mathrm{~nm}$

Peak Quantitation: AREA

Calculation Method: AREA\%

\begin{tabular}{ccccc} 
No. & RT & Area & Area o & BC \\
\hline 1 & 28.200 & 6380092 & 51.633 & BB \\
2 & 32.687 & 5976628 & 48.367 & BB \\
\hline & & 12356720 & 100.000
\end{tabular}

Chrom Type: Fixed WL Chromatogram, $210 \mathrm{~nm}$

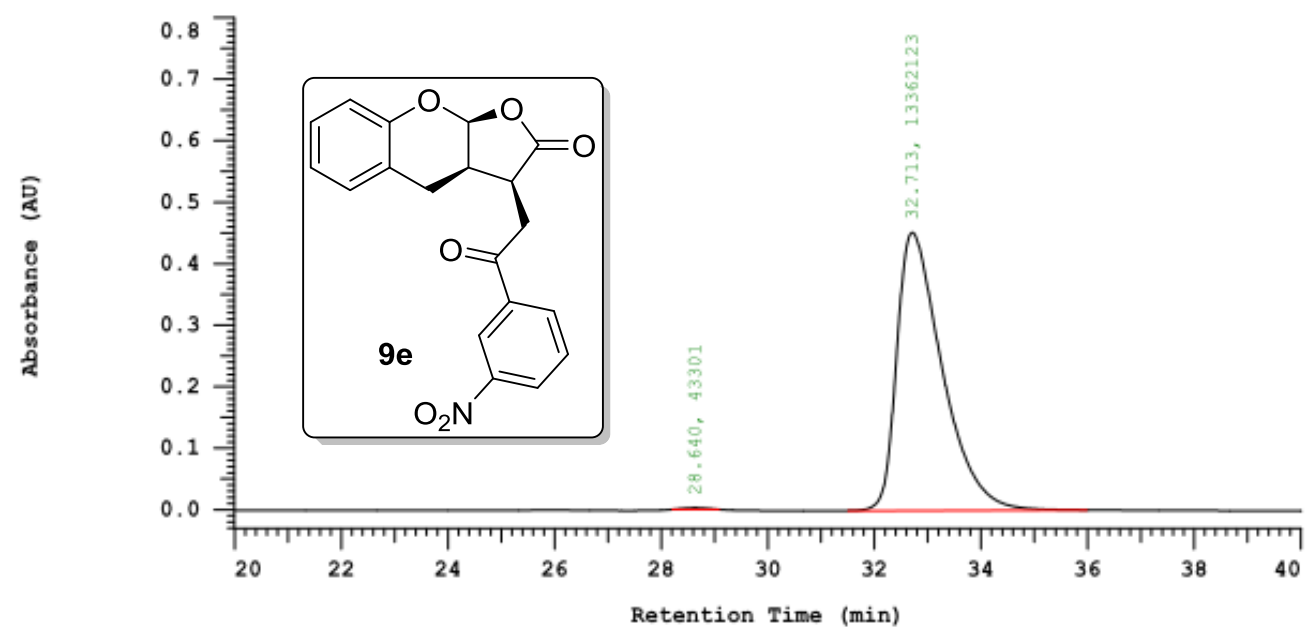

Chrom Type: Fixed WL Chromatogram, $210 \mathrm{~nm}$

Peak Quantitation: AREA

Calculation Method: AREA응

\begin{tabular}{rrrrr} 
No. & RT & \multicolumn{1}{c}{ Area } & Area $\frac{8}{7}$ & BC \\
\hline 1 & 28.640 & 43301 & 0.323 & BB \\
2 & 32.713 & 13362123 & 99.677 & BB \\
\hline & & 13405424 & 100.000 & \\
\hline
\end{tabular}



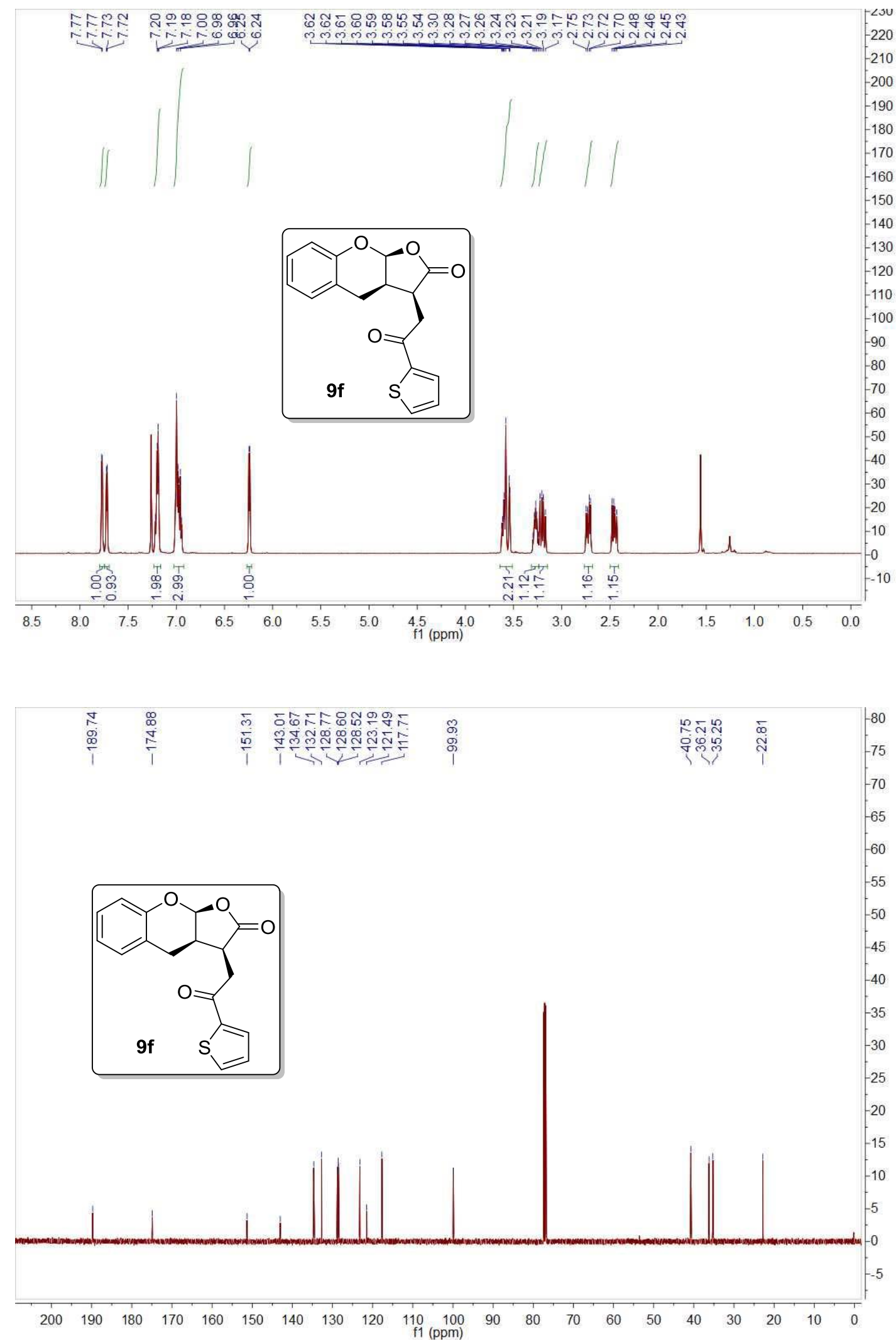
Chrom Type: Fixed WL Chromatogram, $210 \mathrm{~nm}$

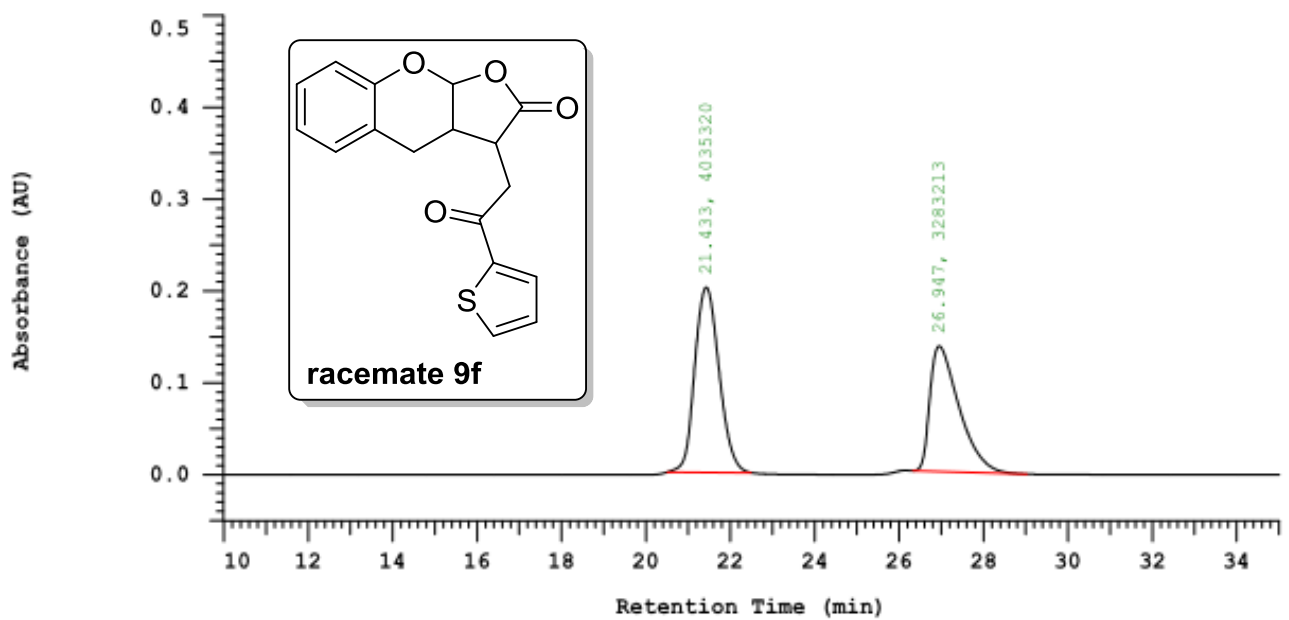

Chrom Type: Fixed WL Chromatogram, $210 \mathrm{~nm}$

Peak Quantitation: AREA

Calculation Method: AREA용

\begin{tabular}{ccccc} 
No. & RT & Area & Area $\frac{8}{5}$ & BC \\
\hline 1 & 21.433 & 4035320 & 55.138 & BB \\
2 & 26.947 & 3283213 & 44.862 & BB \\
\hline & 7318533 & 100.000 & \\
\hline
\end{tabular}

Chrom Type: Fixed WL Chromatogram, $210 \mathrm{~nm}$

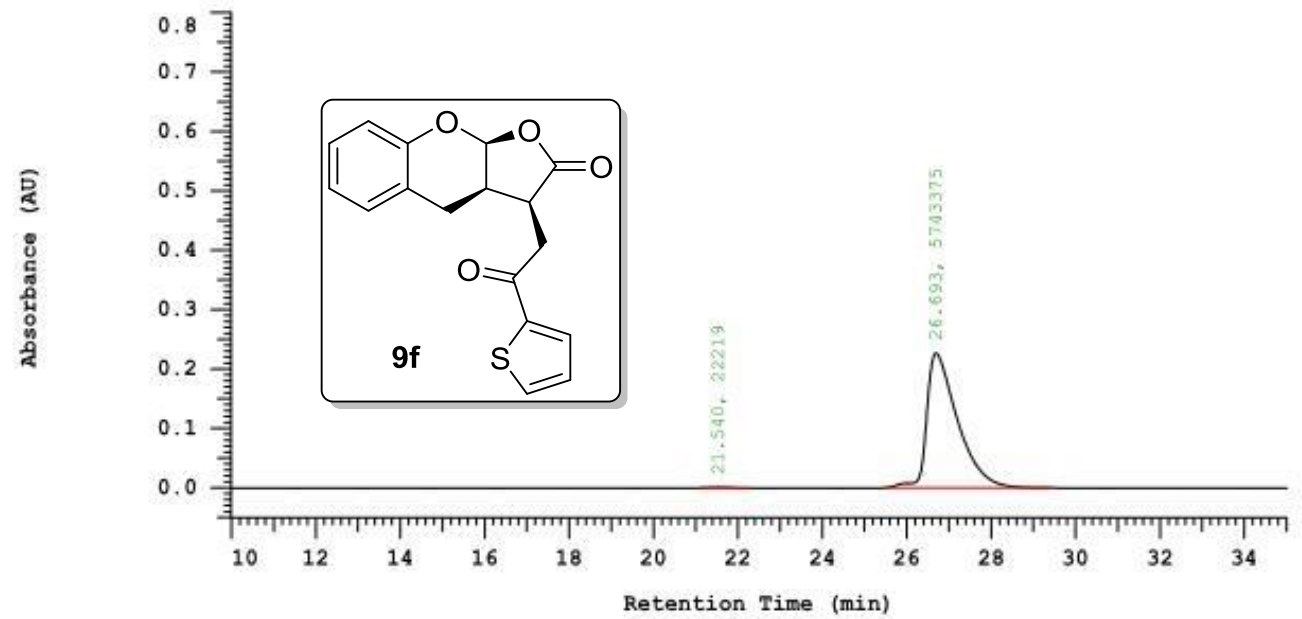

Chrom Type: Fixed WL Chromatogram, $210 \mathrm{~nm}$

Peak Quantitation: AREA

Calculation Method: AREA

\begin{tabular}{|c|c|c|c|c|}
\hline No. & RT & Area & Area \% & $\mathrm{BC}$ \\
\hline 1 & 21.540 & 22219 & 0.385 & $\mathrm{BB}$ \\
\hline 2 & 26.693 & 5743375 & 99.615 & $\mathrm{BB}$ \\
\hline & & 5765594 & 100.000 & \\
\hline
\end{tabular}



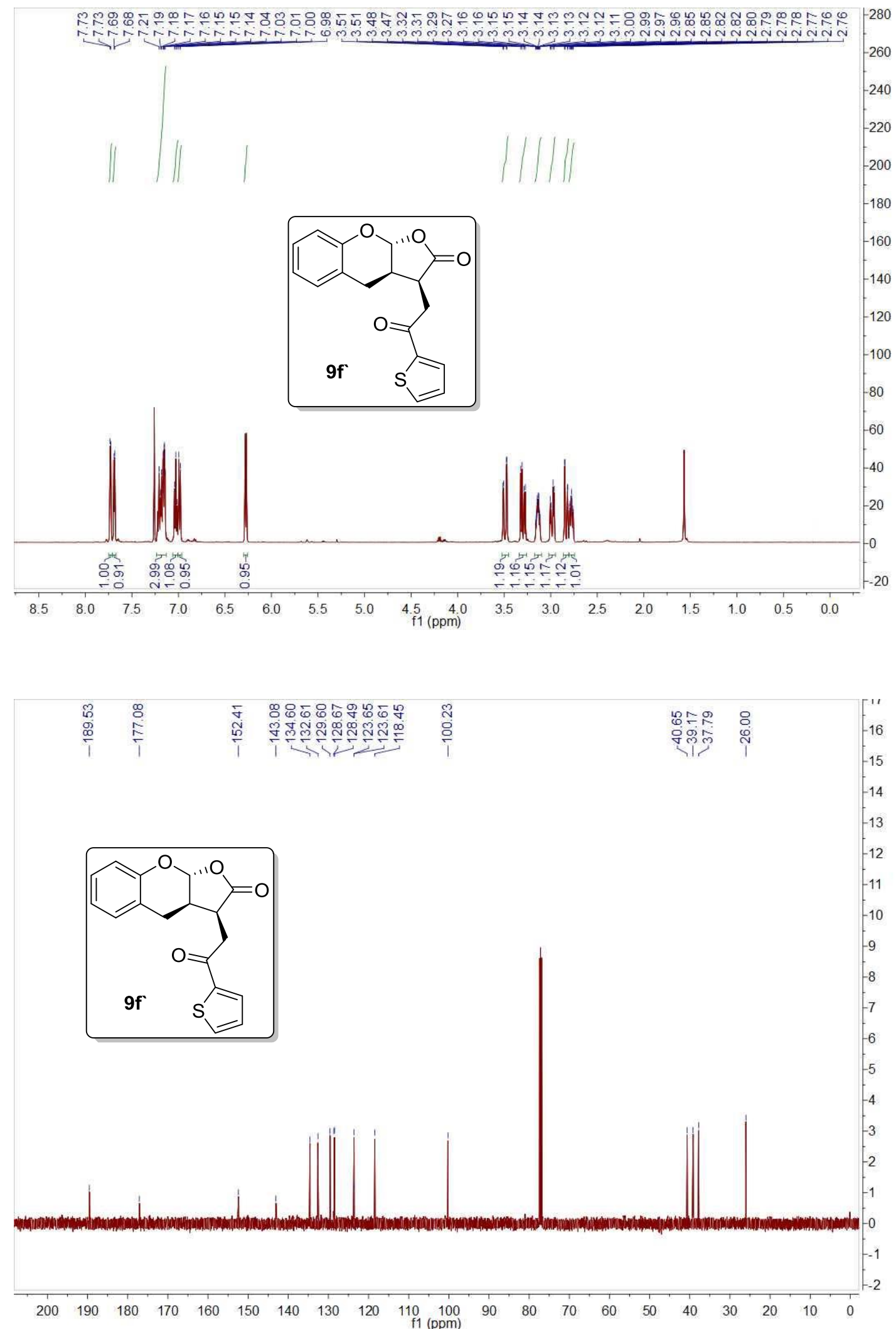
Chrom Type: Fixed WL Chromatogram, $210 \mathrm{~nm}$

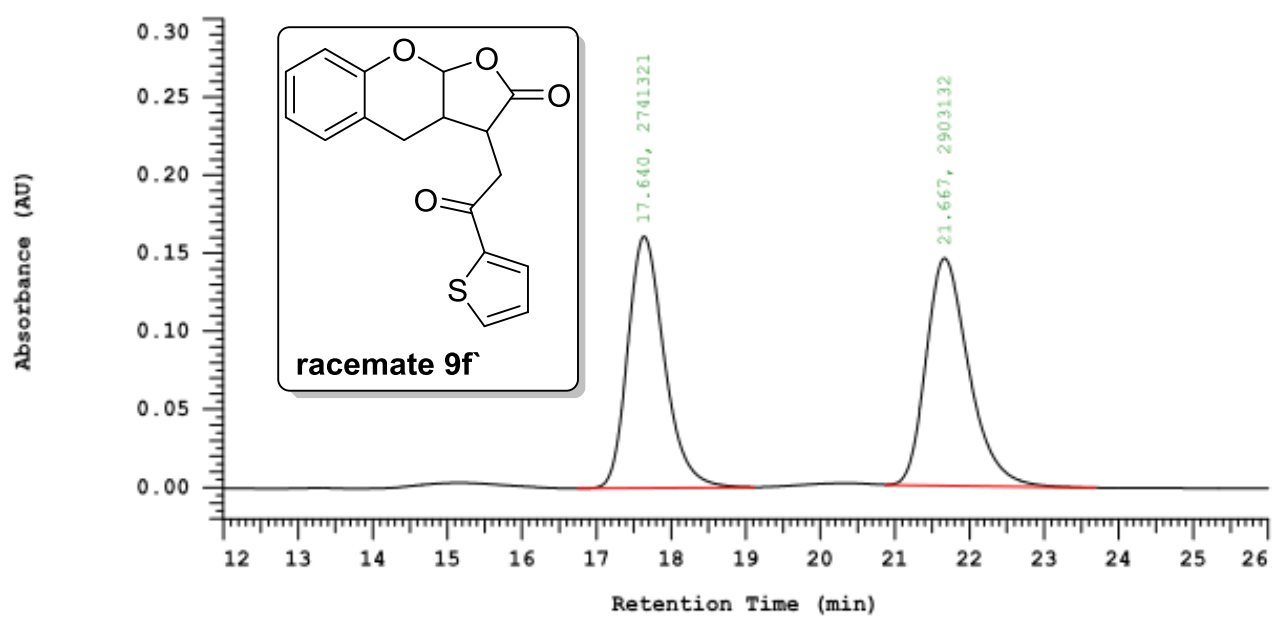

Chrom Type: Fixed WL Chromatogram, $210 \mathrm{~nm}$

Peak Quantitation: AREA

Calculation Method: AREA

\begin{tabular}{ccccc} 
No. & RT & Area & Area 흔 & BC \\
\hline 1 & 17.640 & 2741321 & 48.567 & BB \\
2 & 21.667 & 2903132 & 51.433 & BB \\
\hline & 5644453 & 100.000 & \\
\hline
\end{tabular}

Chrom Type: Fixed WL Chromatogram, $210 \mathrm{~nm}$

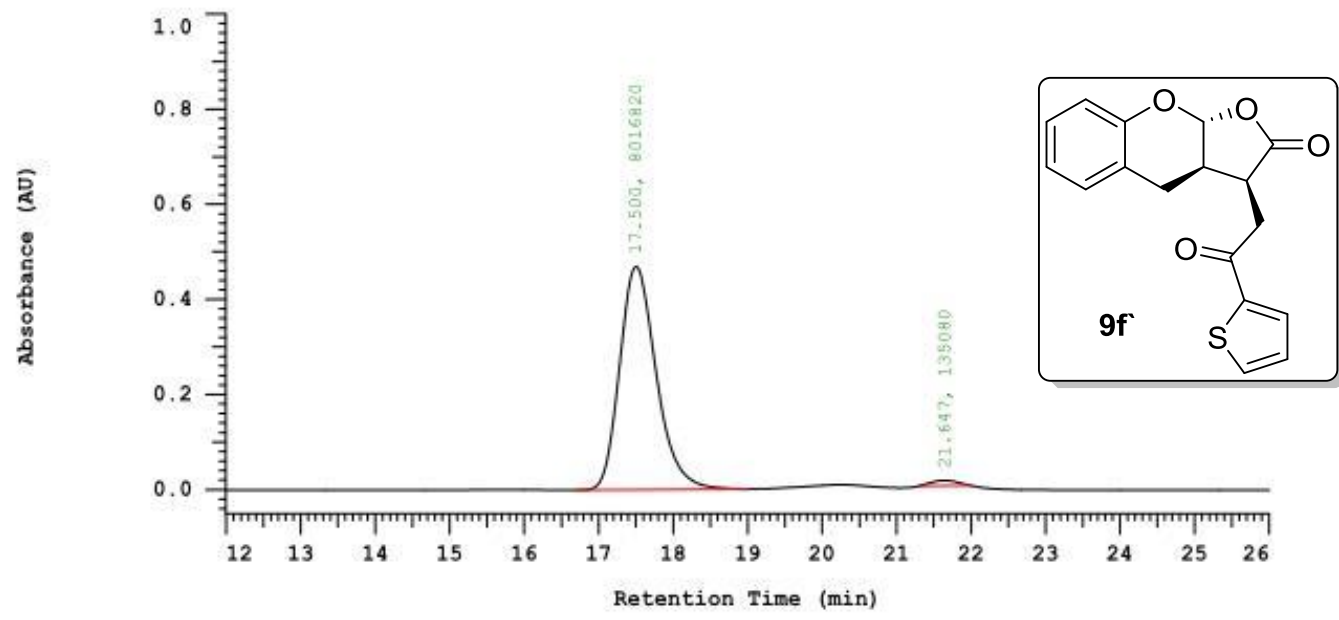

Chrom Type: Fixed WL Chromatogram, $210 \mathrm{~nm}$

Peak Quantitation: AREA

Calculation Method: AREA

\begin{tabular}{|c|c|c|c|c|}
\hline No. & $\mathrm{RT}$ & Area & Area $\frac{\circ}{8}$ & BC \\
\hline 1 & 17.500 & 8016820 & 98.343 & $\mathrm{BB}$ \\
\hline 2 & 21.647 & 135080 & 1.657 & BB \\
\hline & & 8151900 & 100.000 & \\
\hline
\end{tabular}



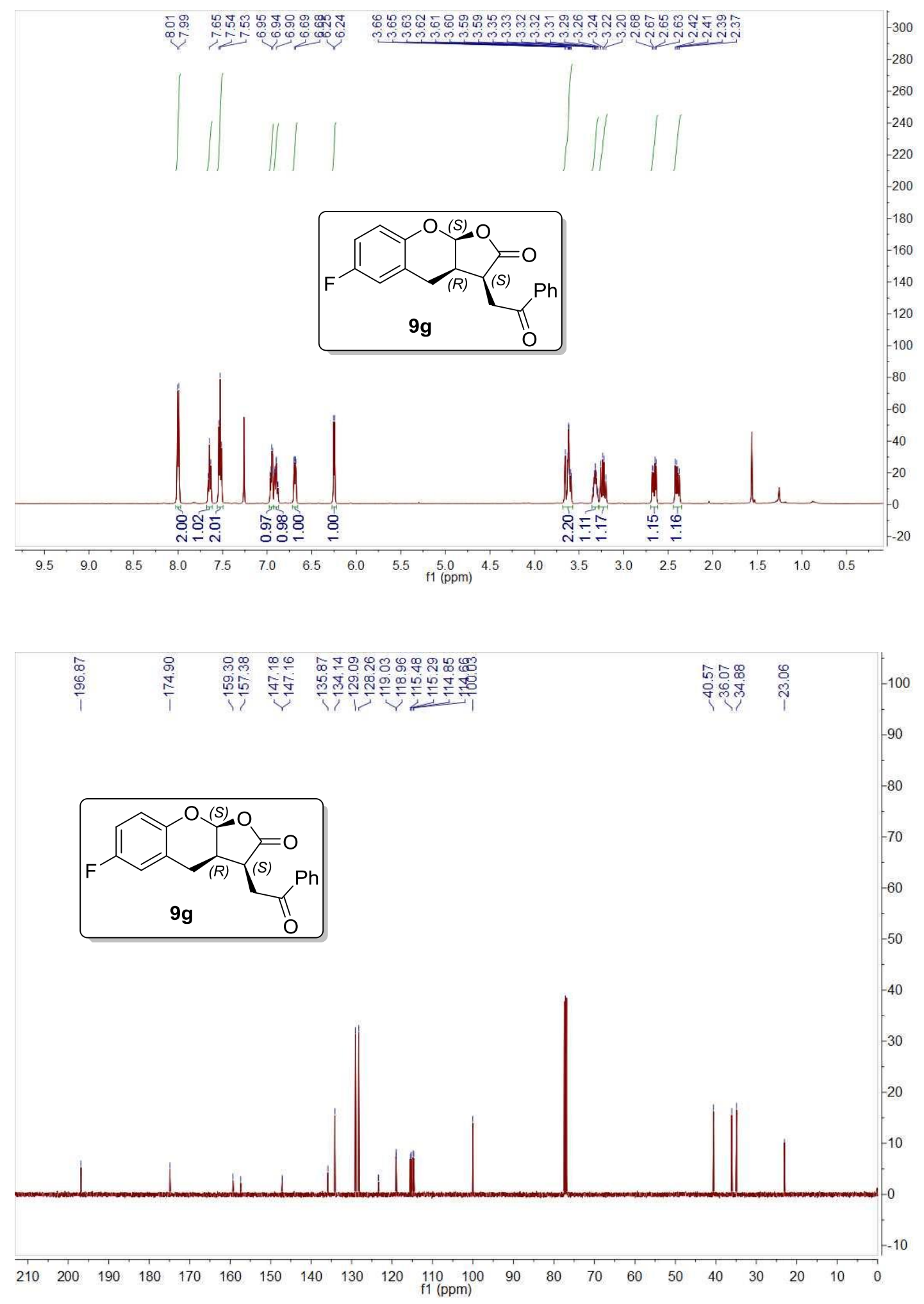
Chrom Type: Fixed WL Chromatogram, $210 \mathrm{~nm}$

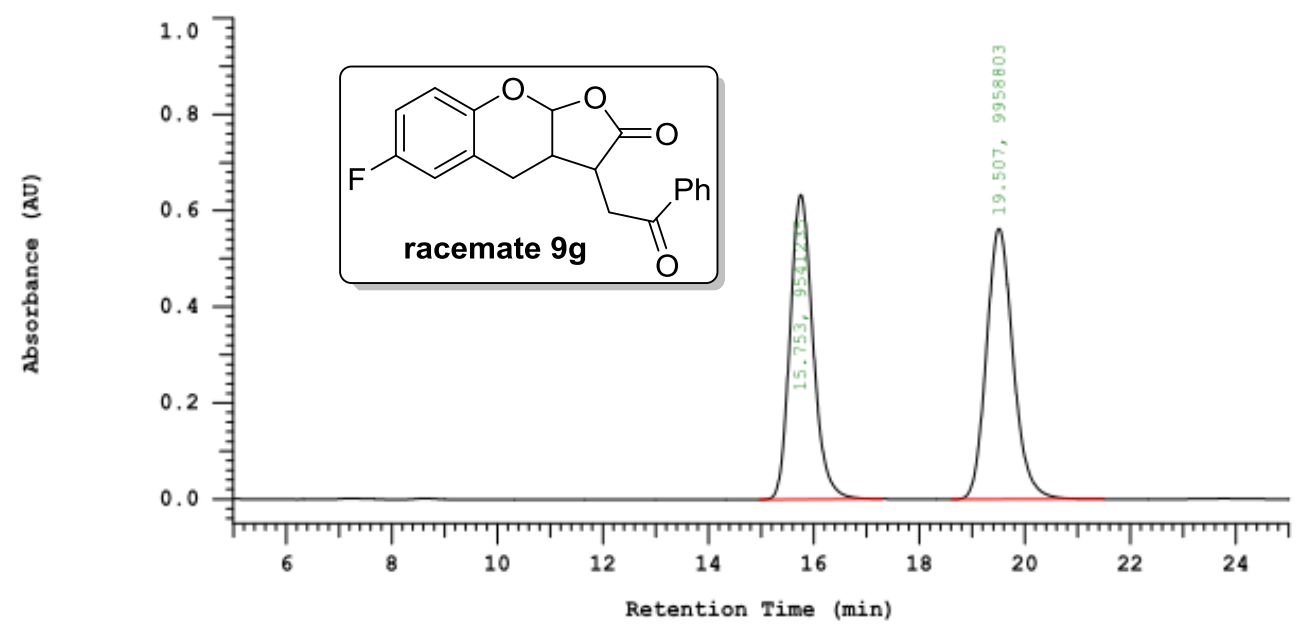

Chrom Type: Fixed WL Chromatogram, $210 \mathrm{~nm}$

Peak Quantitation: AREA

Calculation Method: AREA

\begin{tabular}{ccccc} 
No. & RT & Area & Area o & BC \\
\hline 1 & 15.753 & 9541235 & 48.929 & BB \\
2 & 19.507 & 9958803 & 51.071 & BB \\
\hline & 19500038 & 100.000 & \\
\hline
\end{tabular}

Chrom Type: Fixed WL Chromatogram, $210 \mathrm{~nm}$

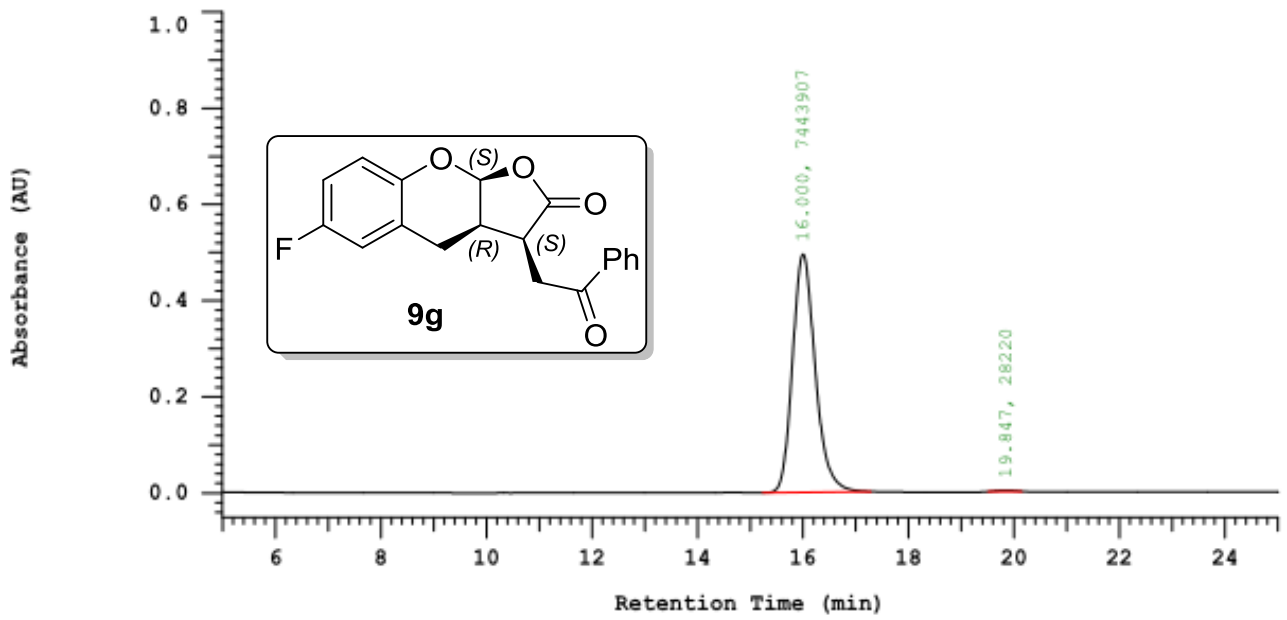

Chrom Type: Fixed WL Chromatogram, $210 \mathrm{~nm}$ Peak Quantitation: AREA

Calculation Method: AREA의

\begin{tabular}{rrrrr} 
No. & RT & \multicolumn{1}{c}{ Area } & Area o & BC \\
\hline 1 & 16.000 & 7443907 & 99.622 & BB \\
2 & 19.847 & 28220 & 0.378 & BB \\
\hline & & 7472127 & 100.000 & \\
\hline
\end{tabular}




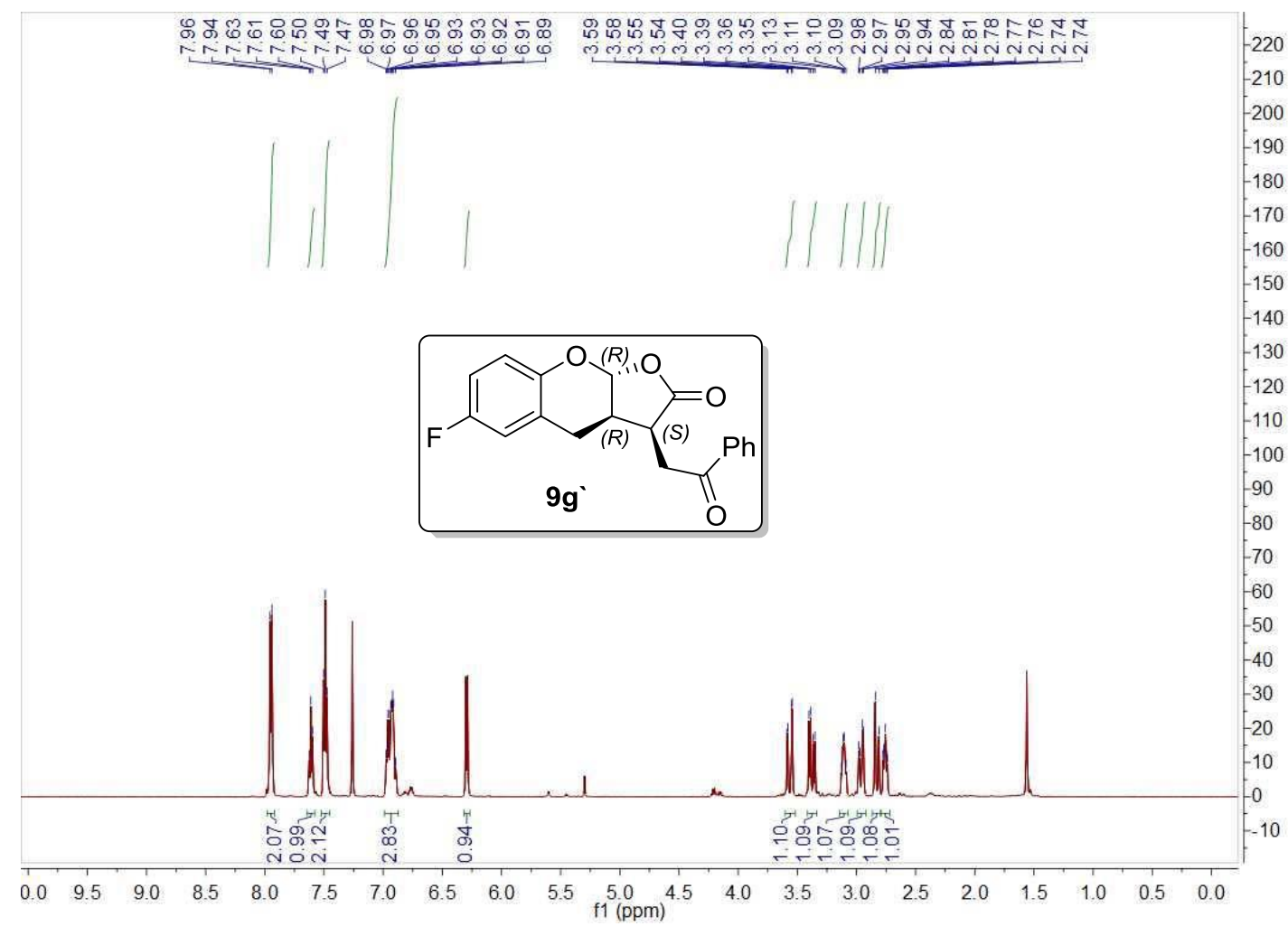

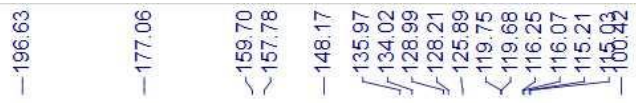

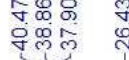

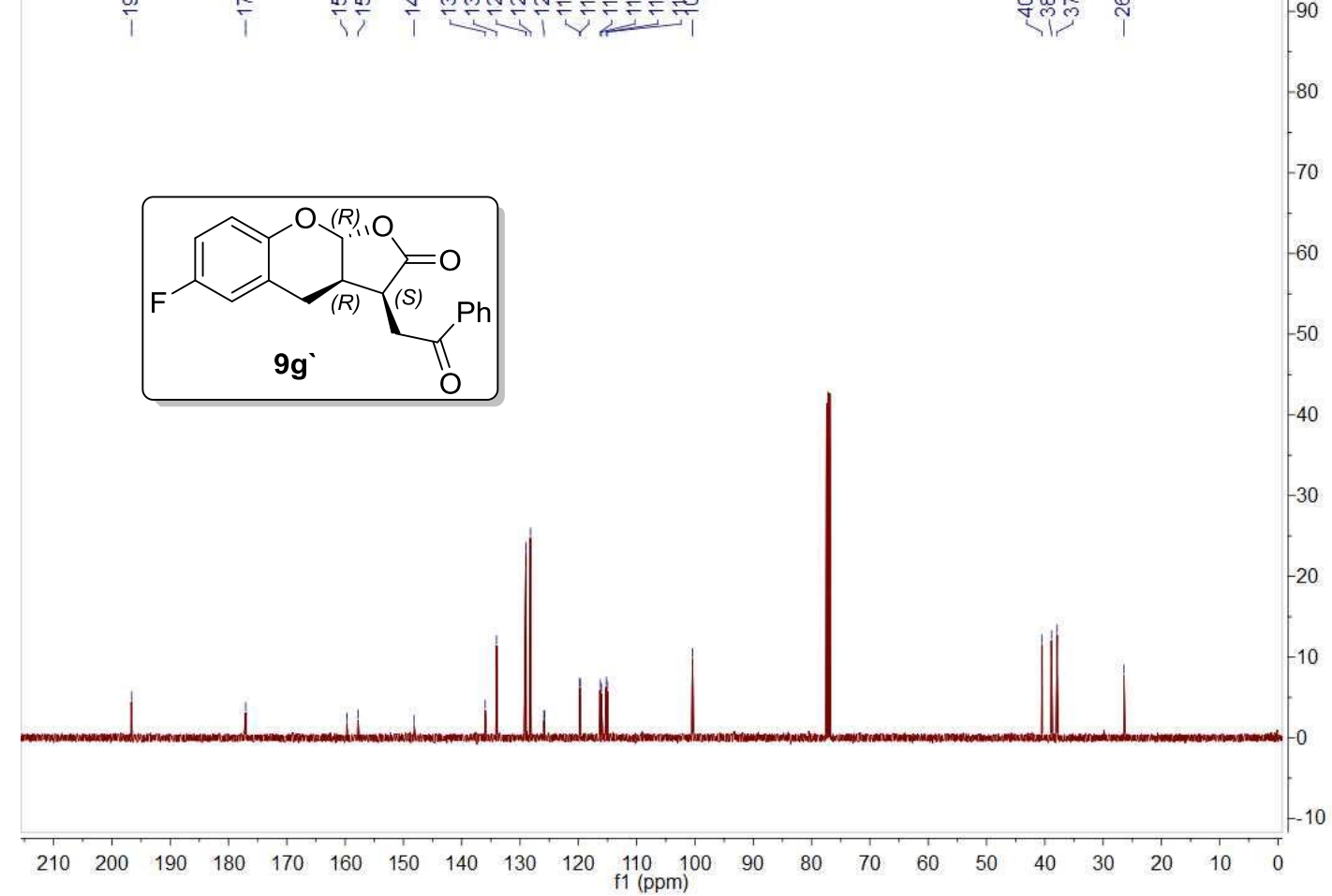


Chrom Type: Fixed WL Chromatogram, $210 \mathrm{~nm}$

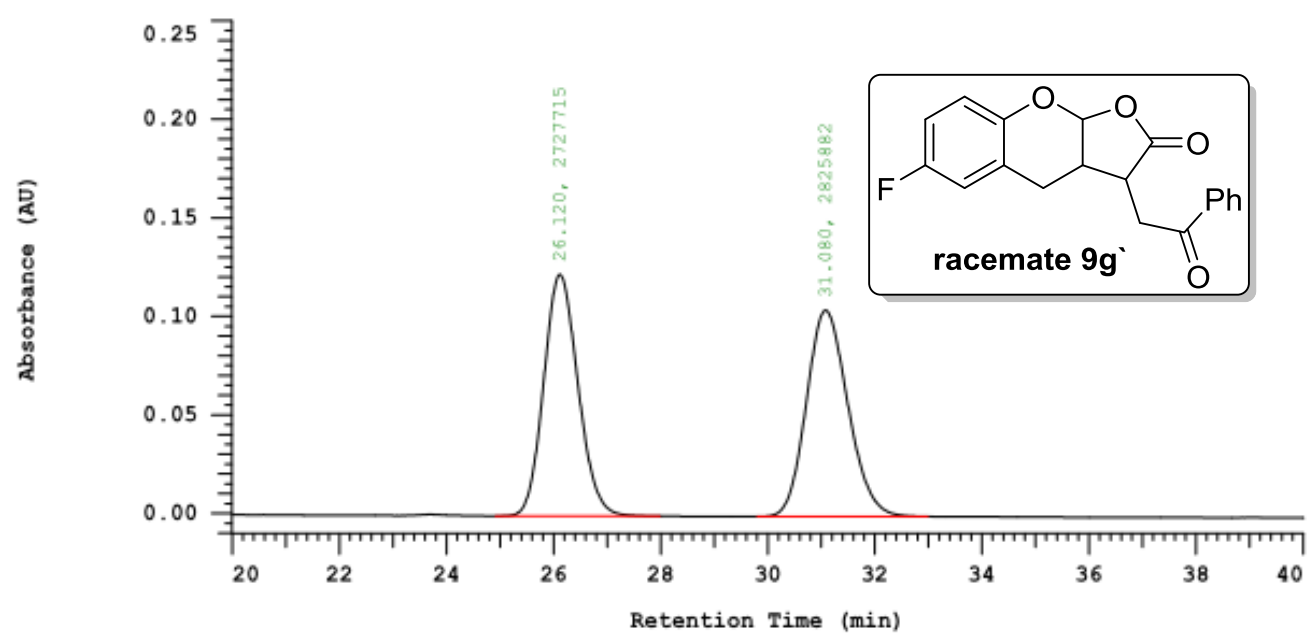

Chrom Type: Fixed WL Chromatogram, $210 \mathrm{~nm}$

Peak Quantitation: AREA

Calculation Method: AREA

\begin{tabular}{ccccc} 
No. & RT & Area & Area $\%$ & BC \\
\hline 1 & 26.120 & 2727715 & 49.116 & BB \\
2 & 31.080 & 2825882 & 50.884 & BB \\
\hline & 5553597 & 100.000 & \\
\hline
\end{tabular}

Chrom Type: Fixed WL Chromatogram, $210 \mathrm{~nm}$

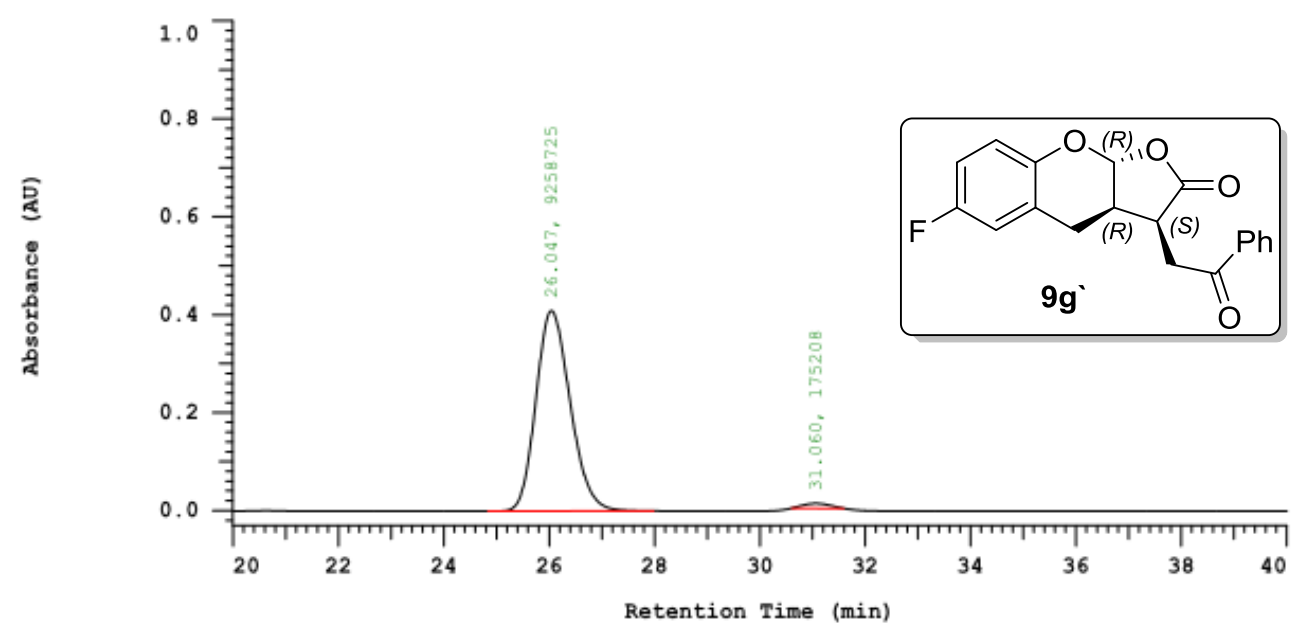

Chrom Type: Fixed WL Chromatogram, $210 \mathrm{~nm}$

Peak Quantitation: AREA

Calculation Method: AREA\%

\begin{tabular}{|c|c|c|c|c|}
\hline No. & $\mathrm{RT}$ & Area & Area 응 & $\mathrm{BC}$ \\
\hline 1 & 26.047 & 9258725 & 98.143 & $\mathrm{BB}$ \\
\hline 2 & 31.060 & 175208 & 1.857 & $\mathrm{BB}$ \\
\hline & & 9433933 & 100.000 & \\
\hline
\end{tabular}

